\title{
ADVANCED BOOST SYSTEM DEVELOPMENT FOR HIGH EGR APPLICATIONS
}

Recipient: Ford Motor Company

Award Number: DE-FC26-07 -NT43280

Working Partners: Ford Motor Company, ConceptsNREC, Wayne State University

Cost-Sharing Partners: Ford Motor Company and Wayne State University

Contact: Harold Sun, 313-248-3077, hsun3@ford.com

DOE Manager: Ralph Nine 304-285-2017 RALPH.NINE@NETL.DOE.GOV 


\section{DISCLAIMER}

This report was prepared as an account of work sponsored by an agency of the United States Government. Neither the United States Government nor any agency thereof, nor any of their employees, makes any warranty, express or implied, or assumes any legal liability or responsibility for the accuracy, completeness, or usefulness of any information, apparatus, product, or process disclosed, or represents that its use would not infringe privately owned rights. Reference herein to any specific commercial product, process, or service by trade name, trademark, manufacturer, or otherwise does not necessarily constitute or imply its endorsement, recommendation, or favoring by the United States Government or any agency thereof. The views and opinions of authors expressed herein do not necessarily state or reflect those of the United States Government or any agency thereof. 


\section{Executive Summary}

Project Title: ADVANCED BOOST SYSTEM DEVELOPMENT FOR HIGH EGR APPLICATIONS

Project Period: October 1, 2007 - September 30, 2012

Date of Report: December 21, 2012

\section{Funding: DOE Share $\$ 1,495 \mathrm{~K}$; Recipient Share $\$ 1,495 \mathrm{~K}$}

\section{Project Objectives:}

- To support industry efforts of clean and efficient internal combustion engine development for passenger and commercial applications

- This program focuses on turbocharger improvement for medium and light duty diesel applications, from complete system optimization percepective to enable commercialization of advanced diesel

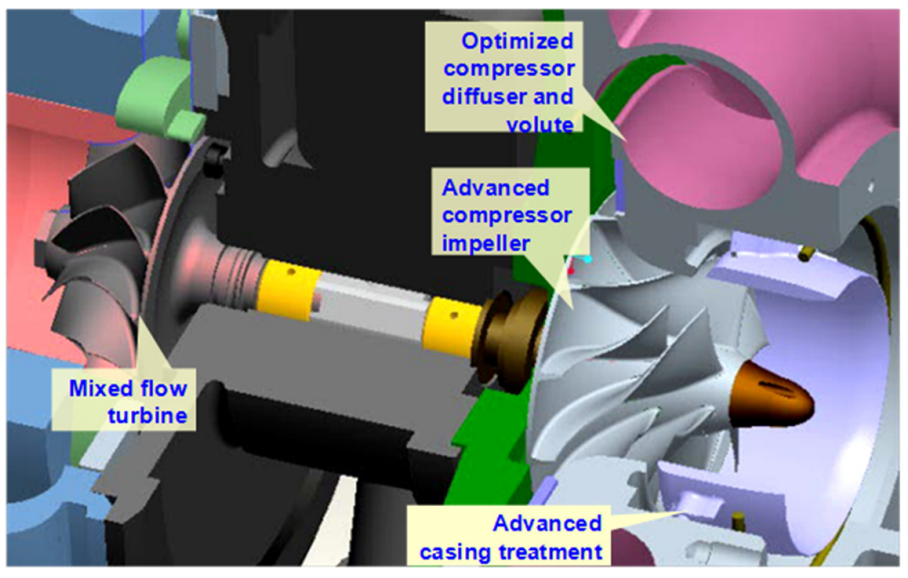
combustion technologies, such as HCCI/LTC.

- Improve combined turbocharger efficiency up to $10 \%$ or fuel economy by $3 \%$ on FTP cycle at Tier II Bin 5 emission level.

Results: Production Honeywell GT35 and IHIF55 turbochargers were used as donor turbo on which advanced compressor and mixed flow turbine wheels were designed and fabricated for MD and LD diesel applications, respectively. Flow bench tests demonstrated that the advanced compressor offers $38 \%$ extra flow capacity, 8-10\% better efficiency over the MD donor turbo compressor. The mixed flow turbine gained $10-15 \%$ efficiency at low $U / C$ areas. The engine dynamometer test has demonstrated that the advanced LD turbo (TS11) gained 3.3\%

FTP cycle thermal efficiency

\begin{tabular}{|r|r|}
\hline IHI Base Turbo & $29.55 \%$ \\
TS11 Adv. Turbo & $30.52 \%$ \\
Improvement & $3.3 \%$ \\
\hline
\end{tabular}
thermal efficiency improvement over the base LD turbo on FTP cycle at T2B5 tailpipe emission level. 
Table of Contents

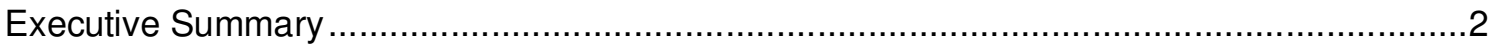

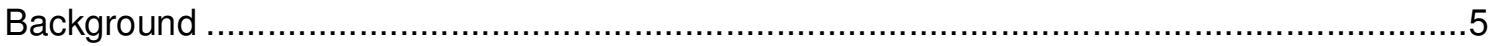

(Task 1. Planning \& Scheduling)

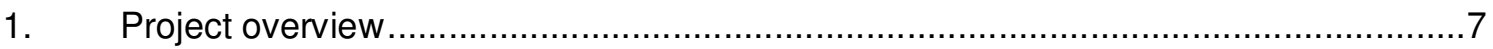

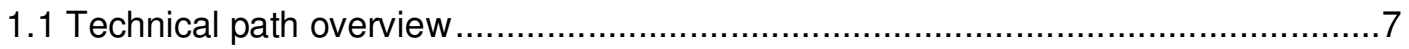

1.2 Description of technical challenges in advanced boost system ...............................

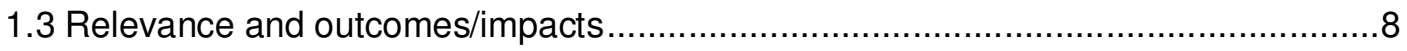

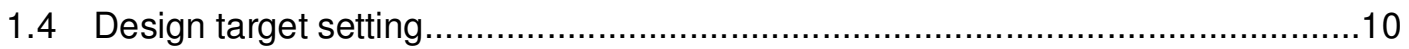

(Task 2. Computer Assessment and CFD analyses)

2. Computer assessment and CFD/CAE analyses for MD turbocharger .....................14

2.1 Computer assessment ................................................................................ 14

2.2 Optimization process ................................................................................... 16

3. Exploratory development: MD turbocharger aerodynamic development ..................19

3.1 Meanline design of the compressor with arbitrary impeller surface (without

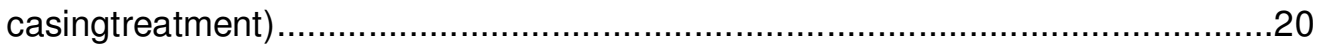

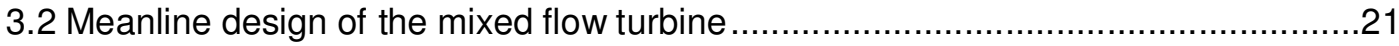

4. Three dimensional designs and analyses of turbocharger for performance

validation and additional design iteration for structure and stress compliances ....................23

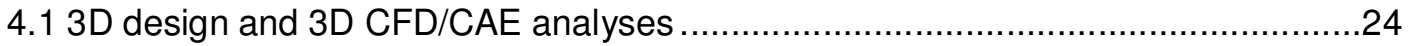

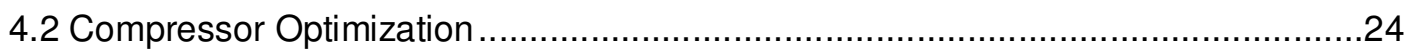

4.2.1 Numerical Model for compressor CFD performance validation ..........................25

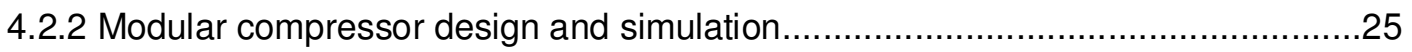

4.3 Numerical investigation of SRCT impact on performance .................................25

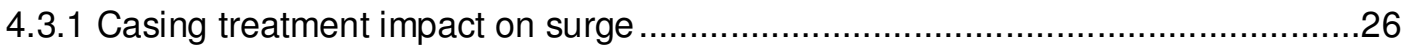

4.3.2 Compressor CFD flow visualization and flow field analysis................................29

4.4 Optimization of choke slot for operation range extension.....................................34

4.5 Optimization of surge slot for optimal low end efficiency and surge margin ............41

4.6 Development of Active Casing Treatment for Wide Range Compressor..................44

4.6.1 Mechanism of single slot and dual slot casing treatment .................................4 44

4.6.2 Conventional Single-Port Casing Treatments ..................................................46

4.7 Stress and structure analyses of the advanced MD Compressor...........................51

4.8 Three dimensional design and CFD/CAE analyses of MD mixed flow turbine ........52

4.9 Rotor dynamics analysis of advanced MD turbocharger assembly .........................58

\section{(Task 3. Control and Actuation System Design and Development)}

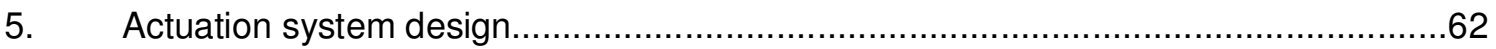

(Task 4. Prototype Fabrication and Flow Bench Test)

6. Fabrication of prototype advacned MD turbocharger. 
7. Steady state flow bench test validation of advanced MD turbocharger

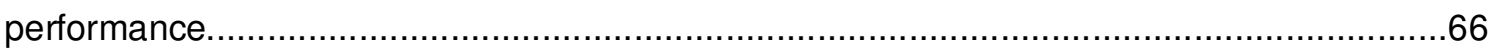

7.1 Steady state flow bench test of advanced MD compressor impeller ....................66

7.2 Experimental Validation of different casing treatment concepts .............................66

7.3 Steady state flow bench test of advanced MD mixed flow turbine ..........................71

8. Steady state MD diesel engine performance test with advanced MD turbocharger ......73

(Task 5. Redesign, CFD Validation, Fabrication and Flow Bench Test of a LD turbo System for LD Diesel Application/Demonstration)

9. Advanced turbocharger development for light duty diesel application............................75

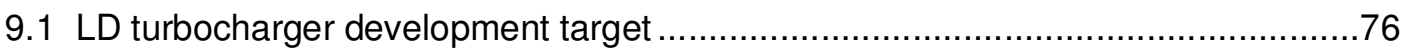

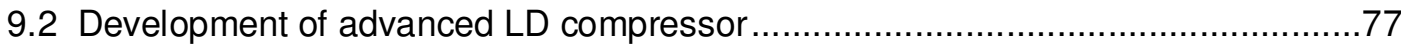

9.3 Development of advanced LD mixed flow turbine ...............................................81

10. Stead state flow bench performance validation of advanced LD turbocharger...........84

(Task 6. Integration and Test Validation on a Multi-cylinder LD Diesel Engine)

11. LD diesel engine performance validation with advanced LD turbocharger

(Task 2 and Task 4 Amendment: Redesign, Fabrication of MD turbocharger with Flank Millable Compressor Impeller and Mixed Flow Turbine)

12. Development advanced MD2 turbocharger .........................................................

12.1 Design and analyses of advanced MD2 compressor.............................................

12.2 Design and analyses of advanced MD2 mixed flow turbine..................................94

12.3 Fabrication of advanced MD2 compressor and mixed flow turbine wheels............98

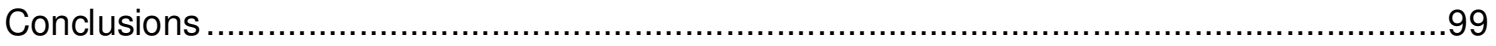

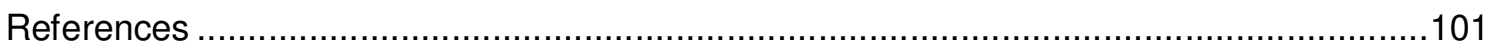




\section{Background:}

Advance combustion technologies, such as $\mathrm{HCCl}$ and LTC, are considered critical to reduce NOx and PM emission on future diesel engines. Over the past twenty years, tremendous effort and progress has been made in the investigation of $\mathrm{HCCl}$ and LTC. Ford Motor Company's internal investigations as well as external researches have demonstrated substantial benefit in NOx and PM reduction as well as BSFC improvement using $\mathrm{HCCl} / \mathrm{LTC}$ and $\mathrm{pHCCl}$ technologies on diesel engines, compared to conventional heterogeneous combustion mode (A. Helmantel, et al, 2004; J. Olsson, et al, 2004; R. Buchwald, et al, 2004; R. Sun, et al, 2004; K. Akihama, et al, 2001; S. Kimura, et al, 2001; S. Kook, et al, 2004 [1, 3, 5, 12, 18, 20, 24, 28]. These Diesel HCCl is based on the concept that if the diesel combustion ignition delay were held long enough, allowing enough time for diesel droplets to be fully atomized, vaporized and mixed with air to form a truly homogenous mixture, spontaneous combustion could occur simultaneously throughout the entire combustion chamber that is heavily diluted by EGR and global combustion temperatures (well below the adiabatic flame temperature due to absorption of thermal energy by EGR that has very high thermal capacity $C_{p}$ ) are thus greatly reduced to levels below the NOx and soot formation threshold.

Investigations by Ford and many other researchers, including Toyota, Mitsubishi, Caterpillar and IFP (Institute Français du Petrole) indicate that simultaneous reduction of soot and NOx is possible with low temperature combustion technology using large amount of cooled EGR under near stoichiometric and even in rich operating conditions.

Different to conventional diesel engine where ignition timing and combustion events are mainly controlled by fuel injection, combustion phasing of a diesel $\mathrm{HCCl}$ engine, is largely kinetically controlled, and depending on the combination of mixture temperature, EGR rate, $A / F$ ratio.

The fundamental of low temperature combustion (or early direct injection $\mathrm{HCCl}$ as others call it) is how to make the overall mixture's chemical delay longer than the physical delay, i.e. to extend the ignition delay so long that onset of combustion does not occur until diesel droplets are fully vaporized and a homogeneous (or sufficiently uniform) fuel air mixture is formed. The fuel air mixture is highly diluted with EGR so that heat release rate is dramatically slowed down (chemical effect) and thermal energy released during the combustion is also absorbed by EGR so the combustion flame temperature remains below the threshold of NOx generation temperature but still higher than the misfire temperature ( 1300K);

Ford researchers have made great progress and successfully demonstrated on single and multi- cylinder diesel engine the early injection, low temperature $\mathrm{HCCl}$ technology. While maintaining similar fuel consumption level as conventional diesel, the 
$\mathrm{NO}_{\mathrm{x}}$ emission was substantially reduced. By using very high level of cooled EGR dilution, the combustion temperature was so low that both NOx and soot formations were suppressed so that the combustion phasing is around TDC to avoid BSFC penalty that most other $\mathrm{NO}_{x}$ reduction technologies would have.

However some key critical technical barriers have prevented the full commercialization of $\mathrm{HCCl} / \mathrm{LTC}$ technology on automotive diesel application. The most important challenges or technical barriers for the full commercialization of $\mathrm{HCCl} / \mathrm{LTC}$ technology on automotive diesel application are related to turbocharger system:

- $\quad$ Almost all of the diesel HCCI/LTC technologies were based on heavy EGR dilution to reduce in-cylinder flame temperature. Therefore driving high level EGR, especially at low engine rpm is critical. However, when a centrifugal compressor is sized to cover rated power operation, it is technically challenging for the compressor to drive high EGR at low engine rpm since the heavy EGR would drive the operation points right into a low efficiency or surge area on compressor as shown in Fig. 1.1.

- $\quad$ Similarly, due to heavy EGR usage, variable geometry turbine would have to work with small vane open position for most of city driving cycles where the turbine efficiency is about 15-30 points lower than its peak efficiency (as shown in Fig. 1.2). It is well understood that both compressor and turbine efficiency would impact transient response, BSFC and, more importantly, turbine out temperature thus aftertreatment efficiencies.

- Diesel $\mathrm{HCCl}$ and LTC technologies greatly reduce $\mathrm{NO}_{x}$ and $\mathrm{PM}$ emission but often at expense of $\mathrm{HC}$ and $\mathrm{CO}$ emissions. It was reported in the literatures that $\mathrm{HC}$ and $\mathrm{CO}$ out of $\mathrm{HCCl}$ diesel could be 20-30 times higher than conventional diesels, [A. Helmantel, et al, 2004 [12]. Although $\mathrm{HCCl} / \mathrm{LTC}$ may substantially reduce $\mathrm{NO}_{\mathrm{x}}$ emissions, it is still likely that $\mathrm{HCCl}$ and $\mathrm{LTC}$ would need aftertreatment for other gaseous emission (e.g. $\mathrm{HC}, \mathrm{CO}$, etc). All diesel aftertreatment devices need a certain temperature to reach a reasonable efficiency. Low turbo efficiency extracts too much energy from diesel exhaust gas; therefore, turbine out temperature for HCCl/LTC could be substantially lower than conventional diesel (could be more than $100 \mathrm{degC}$, according to A. Helmantel, et al, 2004 [12]). The reduced turbine out temperature will compromise the efficiencies of aftertreatment devices.

- The heavy EGR usage in cylinder also causes transient response issue. A truck with 6.0L diesel engine, when diluted with 50\% EGR, will launch with $3.0 \mathrm{~L}$ equivalent engine displacement. A fast response turbo system is essential to quickly bring the engine to full torque. Again, turbo system efficiencies and inertia is critical. It is a widely accepted perception that a series sequential two-stage turbocharger system may help to resolve the aforementioned compressor surge and low compressor/turbine efficiency 
problems. The series sequential turbocharger has a small high-pressure stage turbo to drive high levels of EGR efficiently and crisp response at low to medium engine rpm and load. The turbo system may switch to large size, low-pressure stage turbocharger to have sufficient flow capacity for high power at rated condition. The conclusion would have been true from a performance perspective and without aftertreatment. From an application point of view for future emission compliance, beside cost and packaging issues, an extra turbo also causes extra thermal energy dissipation and excessive warm up time due to extra mass. The thermal energy dissipation inevitably compromises aftertreatment efficiency.

To support and help commercialization of clean, efficient combustion concept, such as $\mathrm{HCCl} / \mathrm{LTC}$ technologies, more advanced diesel boost technologies is critical. The advanced diesel boost system has to be more efficient in $\mathrm{HCCl} / \mathrm{LTC}$ zone while driving high levels of EGR and still has sufficient surge margin to tolerate aggressive engine decelerations. It has to be compact for smaller thermal dissipation for better aftertreatment efficiency and transient response than conventional single stage turbocharger. However, it still has sufficient flow capacity for high power density. Especially, due to high EGR usage, less exhaust gas flowing through turbine, compressor has to be optimized to match the reduced turbine size, i.e. more flow capacity is needed for a smaller compressor wheel.

All these issues and requirements were addressed comprehensively in the study in a systematic approach.

\section{$1 . \quad$ Project Overview:}

\subsection{Technical path overview}

Given more stringent emission regulation with better fuel economy and transient response requirements in the near future, the aforementioned advanced combustion $(\mathrm{HCCl} / \mathrm{LTC})$ technologies are critical path to clean and efficient diesel engines. As mentioned above, advanced diesel boost system has been identified as a key enabler for diesel HCCl/LTC, based on Ford's extensive study in the area in the past few years. Therefore, this study focused on more advanced diesel boost technologies.

\subsection{Description of technical challenges in advanced boost system}

The major challenges to meet future diesel boost requirements include:

- High efficiency in both the turbine and the compressor over a broad operation range, especially at low engine rpm and part load where $\mathrm{HCCl} / \mathrm{LTC}$ is most desirable;

- $\quad$ Sufficient flow capacity and efficiencies for high power density; 
- Compact design and low thermal dissipation for better aftertreatment efficiency;

- Low mechanical inertia for better transient response;

- Low thermal inertia for fast warm up;

- $\quad$ Sufficient surge margin at low engine rpm even with high level of EGR to avoid tip out surge;

- $\quad$ Tolerate "mismatch" between compressor and turbine flow capacities due to high level of EGR requirement at part load conditions;

- Simplicity in control and calibration;

- Better durability/reliability

\subsection{Relevance and Outcomes/impacts}

There are several boosting concepts that have been seen in the literature and potentially be helpful to extend operation range with decent efficiency $[\mathrm{H}$. Uchida, et al, 2006. N. Karmanis, et al. 2002, K. Habermann, et al. 2006 [25, 16, 8]. They are primarily single stage turbochargers so that they are cost effective, have small package space and small thermal inertia while providing enough EGR that is required by advanced combustion concepts such as $\mathrm{HCCl} / \mathrm{LTC}$.

The boost technologies that were investigated under this contract include aerodynamic improvements on both compressor and turbine side:

a. Compressor Variable Inlet Guiding Vane (VIGV). By varying the air rotational flow direction at compressor inlet to have a better alignment with compressor blades, the compressor can work at a smaller mass flow without surge or stall. Toyota's technical report indicated $>30 \%$ improvement in surge margin when a VIGV was used on a conventional compressor [H. Uchida, et al, 2006 [25]. Similar applications have been seen in publication by FEV [Habermann, et al, 2006 [8]. Based on overall air handling design, it is also possible to have an aerodynamically induced prewhirl before the compressor inlet that can extend compressor operation range without a surge [Kyrtatos, N. 1980, Helmut, K. 2004 $[17,13]$. Depending on rotational directions of the VIGV or aerodynamically induced prewhirl, which can be manipulated and controllable, we expected to move the surge line to smaller mass flow area while push the choke line to high mass flow area. Extensive numerical analyses were conducted along with design concepts of VIGV, which concluded that the compressor efficiency was penalized even though the surge margin was substantially improved.

b. Similarly, a well guided recirculation slit on the compressor housing can also realign the re-circulated air flow to extend surge margin. Research shows that up to $29 \%$ surge margin improvement is possible even with a limited compressor efficiency improvement, according to report from Toyota and Honeywell [Gu, 
Ronglei et al, 2004[7]). Later in our research, it was found out that dual slot active casing treatment is even more effective in achieving high compressor efficiency and wide operation range.

c. Variable diffuser compressor. Typically, the impeller exit kinetic energy accounts for $30-50 \%$ of the shaft work input to the compressor stage. To improve compressor efficiency it is critical to recover as much kinetic energy as possible [N Baines, 2005 [4]. According to Baines, the flow leaving the impeller is highly distorted, unsteady and turbulent. Baines also pointed out that potentially vaneless diffusers typically may recover $45-60 \%$ of static pressure while airfoil diffusers may recover $55-75 \%$. An optimized airfoil diffuser can push surge line to lower flow rate on the compressor map, often at the expense of efficiency in offpeak operation range and possibly reduce full flow capacity. A variable vane at compressor diffuser areas can maintain high compressor efficiency at a wide range of operation.

d. Dual sequential compressor volute/outlet. Bifurcated volute that has dual outlet and can be opened sequentially to match air exit velocity to compressor vane geometry. This method is an alternative design concept that is simpler, more economical and potentially more durable than variable geometry compressor mentioned above [Fig. 1.5. J. Yang, et al, 2003 [29] since it has only one moving part: switching valve. Even for vaneless compressors the dual sequential compressor volute/outlet concept may still have advantages over single volute/outlet. Although a surge most likely would occur at the inducer area for vaneless compressors, the volute tongue may still set up a circumferential disturbance that may give rise to a non-uniform pressure field in the inducer, which can cause premature stall or even surge [Fisher, 1988[6]. Its benefit has been analyzed and quantified analytically [30].

e. Mixed flow turbine is an attractive option to improve efficiency on turbine side. Current turbine designs for automotive diesel application are all radial flow based that have narrow flow range and mainly focus on better peak efficiency for high boost and peak torque/power, which may be relevant for heavy duty line haul truck applications. The turbine efficiency at light load and low speed is substantially lower than its peak efficiency. However, for chassis certified diesel application, peak boost may not be a concern since heavy EGR at full load is not required. Instead, high EGR at low engine rpm and light load, where $\mathrm{NO}_{x}$ aftertreatment is not efficient, is required to enable $\mathrm{HCCl}$ or LTC. Therefore, turbine for chassis cert application has to be efficient enough at low end to ensure sufficient turbine out temperature for better aftertreatment efficiency as well as improved BSFC, while maintaining sufficient flow capacity at rated speed for high power output. A mixed flow turbine suits chassis cert diesel applications: lower expansion ratio with high turbine efficiency over wider range [Karamanis, $\mathrm{N}$. 2002 [16]. This reduced sensitivity of turbine efficiency to turbo rotational speed 
enables the mixed flow turbine to better utilize the highly pulsed exhaust energy in automotive applications. ABB has successfully demonstrated the mixed flow turbine technology on their latest design of RR151, a large size, marine application turbocharger [31]. The target of this study focused on high turbine efficiency at lower speed ratio. Therefore, a switching valve may be added in a twin entry mixed flow turbine to further improve low- end turbine efficiency.

Promising as these technologies or concepts have been introduced in the literature, as history tells us, there are always big gaps between the academic research and real industry applications. E.g. the first variable geometry turbocharger was demonstrated on marine diesels in 1924 by ABB [31]. It took over half a century before the variable geometry turbocharger finally showed up in automotive diesels. Another example is that the promising advanced combustion technologies, such as diesel $\mathrm{HCCl} / \mathrm{LTC}$, are still mostly at research stage even though the concept was published more than 20 years ago [P.M.Najt, et al, 1983 [19]. It would be essential to fully investigate these technologies for next generation automotive diesel applications and have them optimized systematically for real world diesel application along with other emission control requirements and technologies (e.g. EGR distribution and aftertreatment), fully validated on engine dynamometer for future emission compliance and compared against other emission control technologies for fuel economy, cost, performance and emission benefit trade-offs.

\subsection{Design target setting}

Extensive numerical study of the aforementioned boost system technologies with multiple design iterations for each compressor and turbine improvement strategy were conducted prior to hardware build, steady state flow bench test and engine dynamometer test. As the first step, Ford worked with ConceptsNREC and Wayne State University in turbocharger mean line analyses, for engine system as well as turbochargers to generate compressor and turbine maps that would meet the engine system performance requirement. Ford provided performance requirement for advanced diesel combustion (mass flow, pressure/expansion ratio and desired compressor/turbine efficiency maps) which was followed by 1D cycle simulation to assess overall performance and emission benefit on light duty diesel applications as well as system compatibility.

Figure 1.1 illustrated the operation points on dynamometer certified HD FTP and chassis certified LD FTP cycles with high pressure EGR on a typical centrifugal compressor map. Many light load operation points fall in low efficiency area with insufficient surge margin. The focus of this study is to stretch this compressor map as wide as possible to have the high efficiency island cover more FTP cycle while still have superior maximum power. 
The turbine speed ratio $(\mathrm{U} / \mathrm{C})$, a non-dimensional number that is often used to characterize the operation condition of a turbine, is defined as

$$
\frac{U}{C}=\frac{U}{\sqrt{2 C_{p} T_{0}\left[1-\left(\pi_{T}\right)^{-0.285}\right]}}
$$

Where, $\mathrm{U}$ is turbine blade tip speed $(\mathrm{m} / \mathrm{s}) ; \pi_{T}$ is turbine expansion ratio.

Figure 1.2 shows that high levels of EGR requires small turbine vane openings, i.e. lower efficiency at light load conditions; lack of turbine power to balance compressor power demand drives the turbine to operate at lower rotational speed, which further reduces turbine speed; higher expansion ratio for EGR driving and higher turbine inlet temperature due to escalated aftertreatment catalysts further drives the turbine operation point to low turbine speed ratio $\mathrm{U} / \mathrm{C}$ area where typically radial flow turbine would deteriorate sharply in efficiency. One other factor is that high turbine pressure due to aftertreatment catalysts leads to high turbine inlet temperature $T_{0}$, further reducing $\mathrm{U} / \mathrm{C}$.

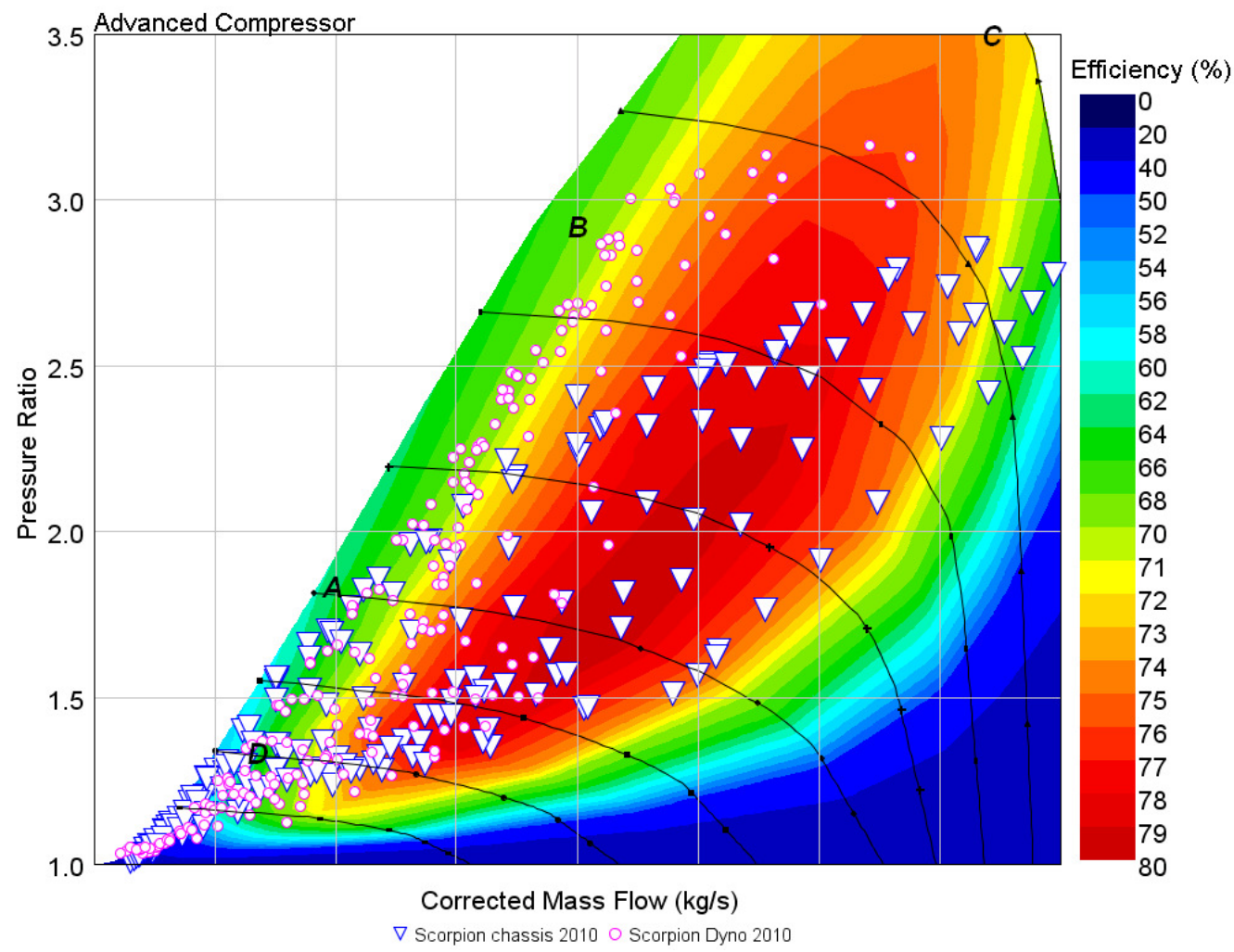

Fig. 1.1. Critical points and engine operation points for chassis cert and dyno cert cycles on the advanced compressor (w/o active casing treatment) 
Turbine Efficiency vs. U/C
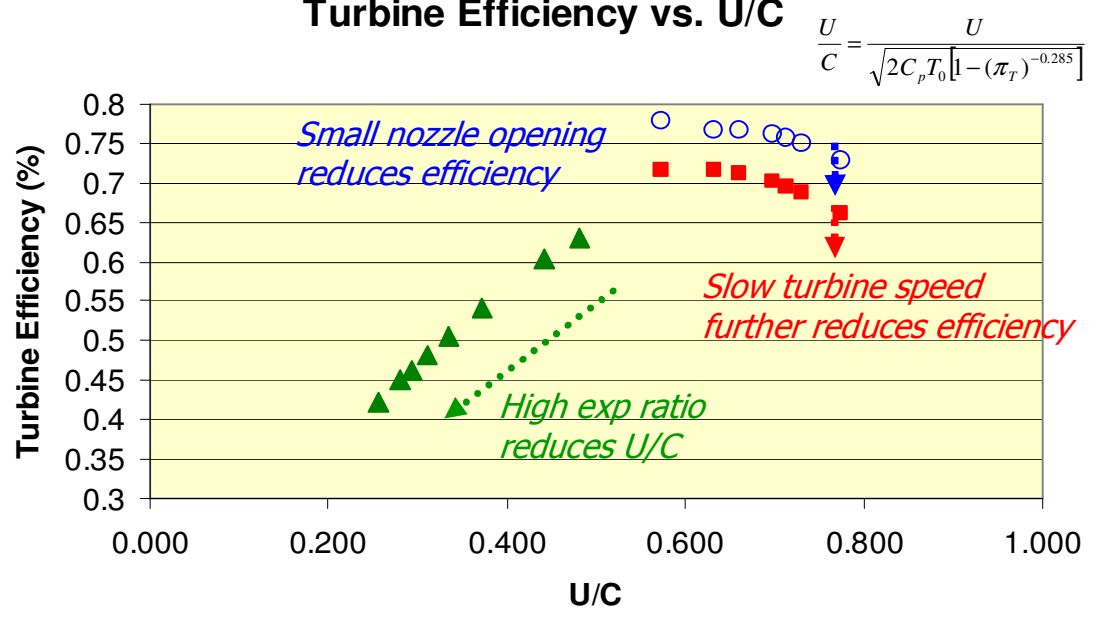

○ $60 \%$ open, high speed $=40 \%$ open, high speed $\Delta 40 \%$ open, low speed

Fig. 1.2. Turbine operation points shifted to low efficiency area due to high level of EGR and high back pressure

Clearly since more and more EGR was used in diesel industry as a major measure to mitigate NOx emissions in the past 15 years, the turbine spent more and more time at low U/C area when operating on FTP cycle (Fig. 1.3). Certainly the high EGR for diesel LTC and $\mathrm{HCCl}$ would only worsen the turbine operation condition. The combination of high EGR, high turbine out pressure due to aftertreatment, thus pushing the turbine to Turbine Speed Ratio (U/C) Distribution over EPA City Cycle operate at low $\mathrm{U} / \mathrm{C}$ area, makes the conventional radial flow turbine less efficient. And we know that the power spent to drive turbocharger can be $30-40 \%$ of engine brake output, i.e. improvement of turbocharger efficiency may have

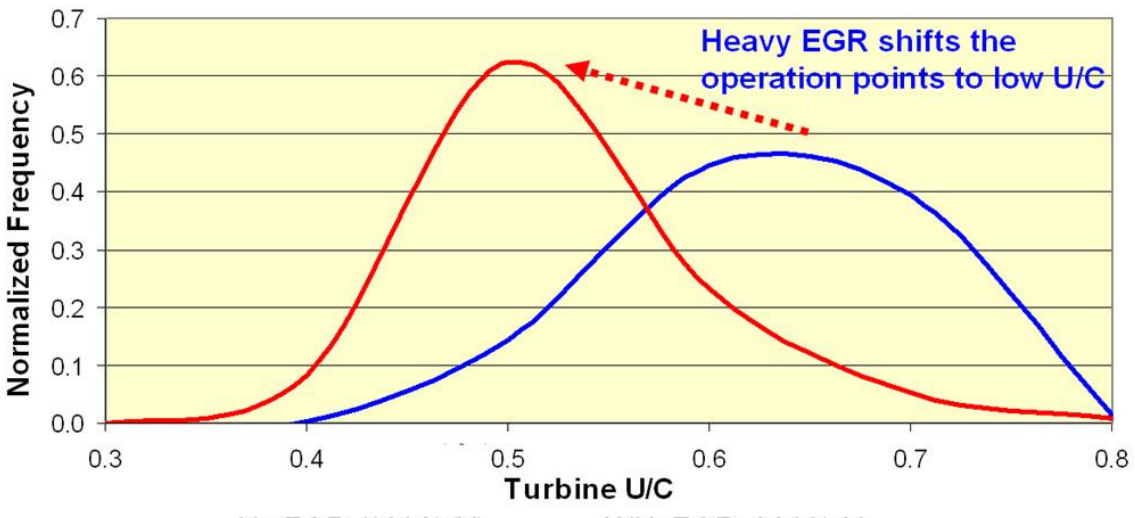
—No EGR (1998MY) —With EGR, 2010MY

Fig. 1.3. Residence time at different turbine speed ratio, 1998MY vs. 2010MY due to adoption of EGR significant impact on engine fuel economy. 
There are two unique areas in this project that may differentiate from other development projects:

- Turbomachinery has overall much longer history than internal combustion engine. Challenging a conventional wisdom may not be easy. However, as the first OEM lead turbocharger development program, the turbocharger can be optimized at vehicle level, i.e. the design targets and priorities can be set with the requirements, constraints and, most importantly but often overlooked, the flexibilities from engines and vehicles. E.g. no one has started a turbocharger development with the visions and boundary conditions for an engine application that is ten years away.

- It is also recognized that computer technologies have made substantial progress in the past three decades, i.e. it is possible to numerically explore in a much bigger design space and a better integrated system that no one has ever tried.

The harsh competition for better performance, lower cost, and government regulation for low NOx and greenhouse gas emission regulations make it more critical to have an advanced turbocharger that can deliver superior power output and improved efficiency over wide operation range with competitive cost. Due to packaging, cost, durability and heat dissipation concerns, the complex and costly two-stage or threestage turbocharging strategy so far has not been a primary path for diesel truck applications, even though the series sequential turbocharger technology has been studied and demonstrated successfully in production vehicles.

The primary focus of this study is to develop an advanced turbocharger that has dramatically improved efficiency and flow range that enables more powerful engine without compromising in fuel economy. The aerodynamic development will be limited to compressor and turbine wheels and housings, i.e. variable geometry turbine (VGT) nozzle and center housing were not part of this research project. Due to the approximation of VGT dimension, a Honeywell production VGT turbocharger GT35 was selected as donor turbo.

A few research partners have participated in the research. ConceptsNREC, with their expertise of design, analyses and development in turbomachinery, has developed and fabricated the turbocharger that has been flow bench tested at Honeywell and BorgWarner. Two visiting scholars at Wayne State University have provided analytical support 3D CFD and CAE support for numerical validation of performance and stress compliance. 


\begin{tabular}{|c|c|c|c|c|c|c|}
\hline \multirow[t]{6}{*}{ Compressor } & Critical points & Priority & \multicolumn{3}{|c|}{ Design Target/1D Prediction } & $\begin{array}{c}\text { Donor compressor } \\
\text { (GT35) }\end{array}$ \\
\hline & & & massflow & PR & efficiency & efficiency \\
\hline & $\mathrm{A}$ & 2 & 0.10 & 1.8 & $70 \%$ & $72 \%$ \\
\hline & $\mathrm{B}$ & 3 & 0.20 & 2.9 & $79 \%$ & $73 \%$ \\
\hline & $\mathrm{C}$ & 1 & 0.37 & 3.5 & $69 \%$ & N/A \\
\hline & $\mathrm{D}$ & 1 & 0.07 & 1.3 & $66 \%$ & $68 \%$ \\
\hline \multirow[t]{6}{*}{ Turbine } & Critical points & Priority & \multicolumn{3}{|c|}{ Design Target/1D Prediction } & Donor Turbine \\
\hline & & & red mass & ER & efficiency & eta*M efficiency \\
\hline & A & 2 & 0.015 & 1.75 & $66 \%$ & $54 \%$ \\
\hline & $\mathrm{B}$ & 3 & 0.020 & 2.58 & $69 \%$ & $61 \%$ \\
\hline & $\mathrm{C}$ & 1 & 0.029 & 2.56 & $73 \%$ & $67 \%$ \\
\hline & $\mathrm{D}$ & 1 & 0.013 & 1.3 & $61 \%$ & $56 \%$ \\
\hline \multirow[t]{6}{*}{$\begin{array}{l}\text { Turbo } \\
\text { Assembly }\end{array}$} & Critical points & Priority & \multicolumn{3}{|c|}{ Design Target/1D Prediction } & $\begin{array}{l}\text { GT35 Donor } \\
\text { Turbocharger }\end{array}$ \\
\hline & & & massflow & Cmp PR & efficiency & efficiency \\
\hline & $\mathrm{A}$ & 2 & 0.10 & 1.8 & $46 \%$ & $39 \%$ \\
\hline & $\mathrm{B}$ & 3 & 0.20 & 2.9 & $55 \%$ & $45 \%$ \\
\hline & $\mathrm{C}$ & 1 & 0.37 & 3.5 & $50 \%$ & N/A \\
\hline & $\mathrm{D}$ & 1 & 0.07 & 1.3 & $40 \%$ & $38 \%$ \\
\hline
\end{tabular}

Table 1.1. Design targets, compared with donor turbo (journal bearing) and 2010MY Scorpion chassis cert turbocharger (ball bearing)

It was well understood that engineering is a trade-off between often conflicting requirements over entire operation range for automotive applications, i.e. single point design target is not sufficient to represent the complete and highly dynamic engine system. In this study, four operation points were selected to be refined as design targets (A, B, C, D points in Fig.1.1). With the multiple iterations between turbocharger meanline analysis (using ConceptsNREC's Compal and Rital) and GTPower engine performance simulation, the turbocharger performance targets listed in Table 1.1 were set that were considered necessary in order to achieve $3 \%$ fuel economy improvement over FTP cycle.

\section{Computer Assessment and CFD/CAE analyses for MD turbocharger:}

\subsection{Computer assessment}

To optimize the turbocharger system that meets engine performance requirement, while improves the turbocharger efficiency over the operation range that is substantially wider than current production turbocharging technologies, the first step is to use one 
dimensional cycle simulation to define engine system requirement, which was cascaded to compressor and turbine design target with focus of the following areas (Fig. 2.1):

Compressor: impeller; casing treatment; diffuser; volute

Turbine: mixed flow turbine wheel, volute

Like any engineering problems, development of a turbocharger will be a process or trade-off between often conflicting design targets (i.e. customer demands, government regulations, e.g. choke flow capacity, efficiency, transient response and surge margin, etc.), and multiple constraints, such as material strengths, durability requirements, manufacturing cost and most importantly, the design of existing variable geometry turbine nozzle mechanism of the donor turbocharger. Typically, when a turbocharger is "stretched" to cover wide operation range, i.e. to have high choke flow capacity for more power, the efficiency at low end, especially when high amount of EGR is used, and surge margin will be negatively affected.

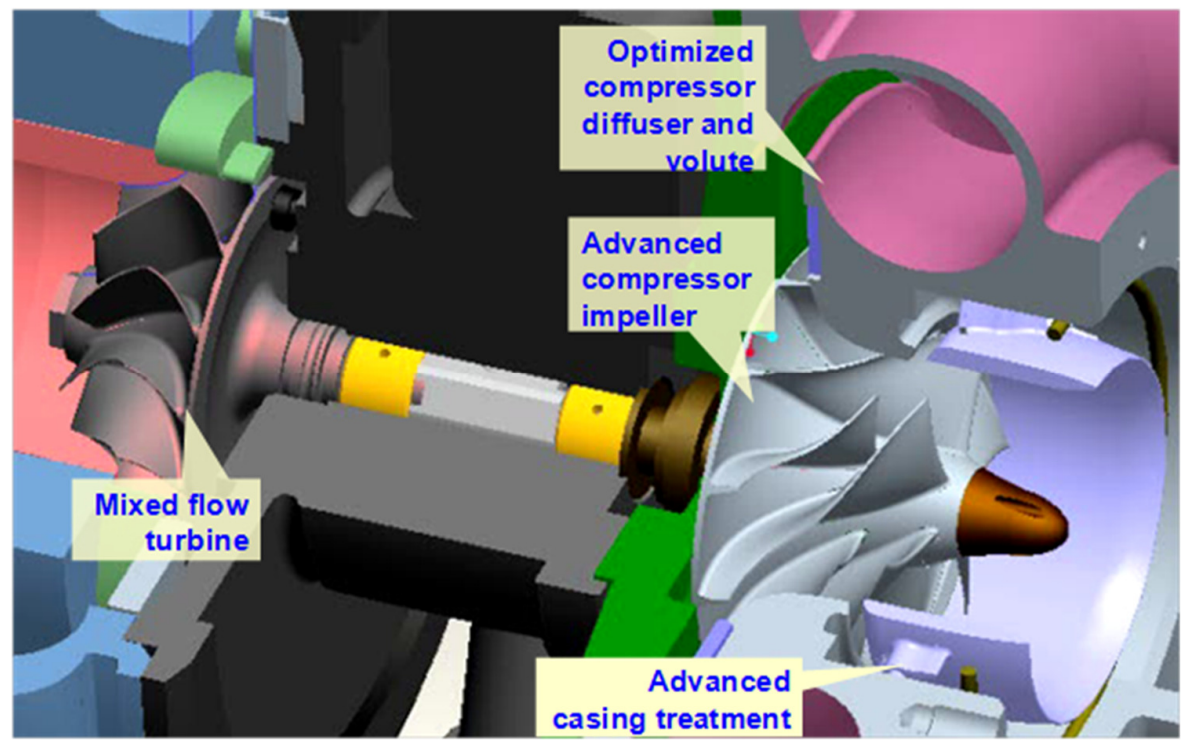

Fig. 2.1. This project focuses on the aerodynamic optimization of compressor impeller, casing treatment and mixed flow turbine

Table 1.1 listed the design targets for this advanced turbocharger. Envisioning more stringent emission standard and upcoming greenhouse regulation, the turbo efficiency at low end where the customer driving cycle are populated, i.e. the "D" point, was set at top priority. On the other hand, the engine rated power or efficiency at peak flow capacity (the "C" point), as an important marketing competitiveness requirement, was also set at top priority. There might be misperception that the turbo efficiency at peak flow capacity is not critical since customer spends little time at rated power thus fuel consumption at rated power would not be important. The fact is: if both compressor and turbine running near choke condition with poor efficiencies, more thermal energy is 
needed to drive the turbocharger, thus the engine will operate with high pumping loss and lower brake power output due to turbine inlet temperature constraint. In other words, turbine inlet temperature limited engine rated power would be increased should the compressor and/or turbine operates near choke condition with higher efficiencies. The efficiency of turbocharger system matters, at low end as well at high end.

The point "A" and "B" were added to anchor the compressor for surge margin and secure the efficiencies near surge area. It is anticipated that high level EGR needed for future stringent $\mathrm{NO}_{x}$ emission control will push the operation points off the peak efficiency area and towards surge line (Fig. 1.1) on a compressor map.

The design targets were set based on a preliminary 1D analysis with the assumption of variable geometry compressor and forward sweep mixed flow turbine for a future high horse power and low $\mathrm{NO}_{x}$ and $\mathrm{CO} 2$ emission engine. Since the VGT and center housing will be carry-over from production GT35 donor turbo, the advanced turbocharger will have a journal bearing. All the efficiency targets at these "critical points" are significantly higher than the donor turbo. The benefit of a ball bearing, which is not the subject of this research, will be additive to the aerodynamic improvement of the advanced turbo, except minor changes in engine calibration, should the ball bearing be adopted in future center housings.

The design target at "C" point has pressure ratio 3.5 that is protected for potential future dyno cert diesel application. The intention was to have dyno cert and chassis cert diesel share the same turbocharger to save manufacturing cost. Certain type of "variable geometry" compressor technology was expected to stretch this map for additional $10 \%$ mass flow.

The donor turbo GT35 was originally designed for a heavy duty engine that has low rated power thus the compressor has a choke flow capacity much lower than 0.37 $\mathrm{kg} / \mathrm{s}$ (thus the efficiency is listed as "N/A" in Table 1)

Initial compressor strategy for high efficiency over wide operation range was focused on the following six areas:

- Optimization of impeller

- Variable compressor inlet guide vane

- Casing treatment

- Guide vanes inside casing treatment

- Variable guide vanes inside diffuser

- Dual and switchable diffuser/volute

After a few iterations of $1 \mathrm{D}$ and 3D numerical studies, the optimal impeller design, casing treatment, guiding vanes inside casing treatment were selected to be pursued. Other technologies, like variable compressor inlet guide vane (with compromise of compressor efficiency), variable guide vanes inside diffuser (narrow operation range for each vane angle, potential complexity in engine calibration and vane coking issues), 
dual/switchable diffuser/volute (packaging difficulty for small size turbochargers), were eliminated from the list.

As EGR was widely used as an effective measure to control feed gas $\mathrm{NO}_{x}$ emission of diesel engines since 1998, the turbine has been seen to spend more time in low U/C area (Fig. 1.3). Conventional radial flow turbine has peak efficiency with U/C between 0.6 - 0.7 (Watson, et. al. 1982, Whitefield, 1990 [32, 34]). Clearly a mixed flow turbine would help to maintain high efficiency at low U/C.

Mixed flow turbine has long be recognized [Wallace, 1971, Watson, 1982 [33, 32] that can shift the high efficiency zone to low $U / C$ area, even though the peak efficiency area may not necessarily be higher than a radial flow turbine. The intention by the time was focused on better utilization of pulsation energy out of engine exhaust, i.e. the instantaneous high pressure pushes operation point of the turbine into low $\mathrm{U} / \mathrm{C}$ area where mixed flow turbine has been demonstrated outperforming conventional radial flow turbine. The benefit of mixed flow turbine under pulsation flow was mostly recognized in academia since most turbo suppliers quantify turbine performance out of a turbocharger assembly on a steady state flow bench, i.e. the turbine $\mathrm{U} / \mathrm{C}$ measurement is limited to a very small area by compressor stable operation range. When Ford started this advanced turbocharger research in 2007, the mixed flow turbine was selected as a key technology due to a totally different reason, i.e. heavy EGR required for future emission compliance also requires high expansion ratio thus low $\mathrm{U} / \mathrm{C}$ for extended of time. The turbocharger suppliers may not have the vision early in their research program that the future NOx emission control, i.e. high EGR usage, has pushed the operation point out of the high efficiency zone of a conventional radial flow turbine. Therefore having OEM lead or at least involve at very early stage of a turbocharger development to ensure a seamless integration of a boost technology into future emission control strategy is essential and beneficial for both supplier and OEM. 


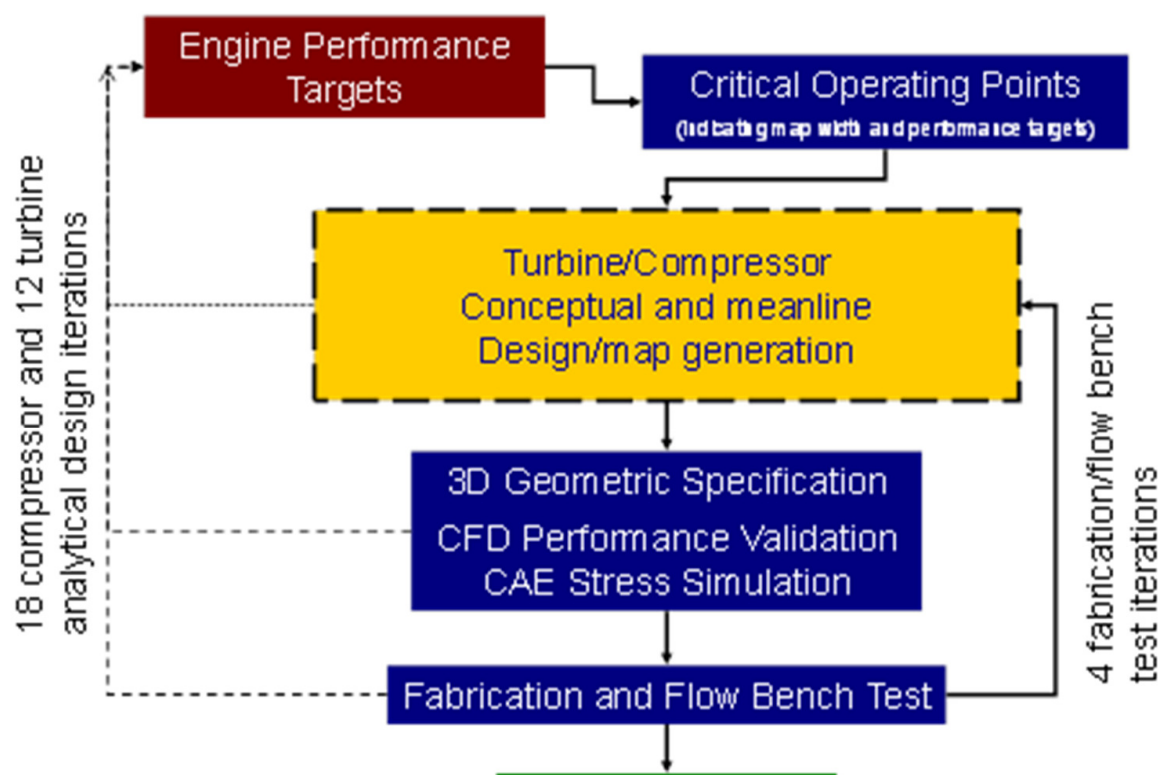

Engine Dyno Test

Fig. 2.2 Design iteration process for turbocharger design optimization and performance validation

\subsection{Optimization process}

As illustrated in Fig. 2.2, the design process starts with engine system level performance targets, or assumptions, which is based on the vision of future engine emission control and fuel economy requirement. A four point performance target as shown in Table 1.1 was proposed out of GTPower engine system simulation, representing heavily weighted part load operation points on customer driving cycle (D), maximum power point $(\mathrm{C})$, and two other points to anchor the compressor for surge margin protection. Compal and Rital (part of ConceptsNREC Agile Suite) were used as meanline, one dimensional design tools for compressor and radial/mixed flow turbine, respectively. Compal and Rital meanline models generated compressor and turbine maps to be validated for engine performance with GTPower simulation. Compal and Rital models were further converted into three dimensional models via AXCENT (another module of Agile Suite) that will be used for 3D CFD performance validation and 3D CAE stress analyses to ensure high cycle fatigue (HCF) and low cycle fatigue (LCF) compliance. The extensive usage of 3D CFD analyses is more than numerical validation of aerodynamic performance of compressor and turbine, especially in the area that meanline models cannot accurately predict. The depiction of flow field in the flow passage actually revealed very valuable insight for additional design optimization as well as new ideas for performance enhancement. 
Over past four years, total 18 compressor design iterations and 12 turbine design iterations were made between these different design stages on the MD turbocharger optimization alone. Four compressor wheels were fabricated and flow bench tested. Two sets of mixed flow turbine wheels were fabricated and flow bench tested. Finally one set of turbocharger was tested on engine dynamometer.

There were two purposes in the fabrication and flow bench testing of prototype compressor and turbine:

a. computer model calibration and correlation;

b. performance demonstration and validation.

The calibrations of numerical models, either 1D or 3D models, are very critical. That is because that all the numerical models were built out of prior experiences with different design features, boundary conditions and applications with the simplification and understanding of physics have yet to be improved under new circumstances. This is especially critical for one dimensional meanline turbo modeling which is often considered more of the art than the science in some cases. It is also true for some well-established, three dimensional numerical analyses, such as computational fluid dynamic (CFD) analyses that are relied on turbulence models and wall functions that may carry some degree of numerical uncertainty and are highly application specific and need to be calibrated.

\section{Exploratory development: MD turbocharger aerodynamic development}

After 1D cycle (GTpower) simulation of engine system in Phase I, subsystem requirements were cascaded for subsystem development. A production donor turbocharger, Honeywell GT35, was selected for medium duty (MD) diesel applications. The variable geometry turbine mechanism remains unchanged during the study even though the mixed flow turbines were used and had to fit in a nozzle vane geometry that was designed for radial flow turbine. During Phase II, Ford and its partners designed a few options of advanced impeller with arbitrary and rule surface with a dual slot/active casing treatment, an actuation mechanism for the active casing treatment, and mixed flow turbine that matched to existing variable geometry nozzle vane design space.

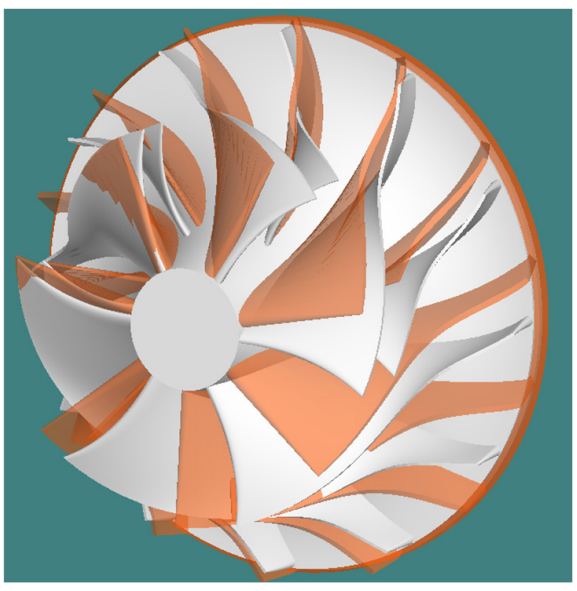

Research in Phase II also includes mechanical design, performance and stress analyses and actuation and control system development. Based on actuation system designs, cost and production feasibilities were carefully assessed with suppliers.

Fig. 3.1 Comparison of a conventional (red) and the advanced impeller 
3.1 Meanline design of the compressor with arbitrary impeller surface (without casing treatment)

\section{Meanline}

design is the first

step of

turbocharger

impeller

development

process. In order

to minimize the

flow losses in the

low mass flow

area, large beta

angle (blade

angle relative to

rotational axis of

the impeller and

bow forward

leading edge

impeller with

arbitrary surface is
Compressor Blade Thickness Distribution

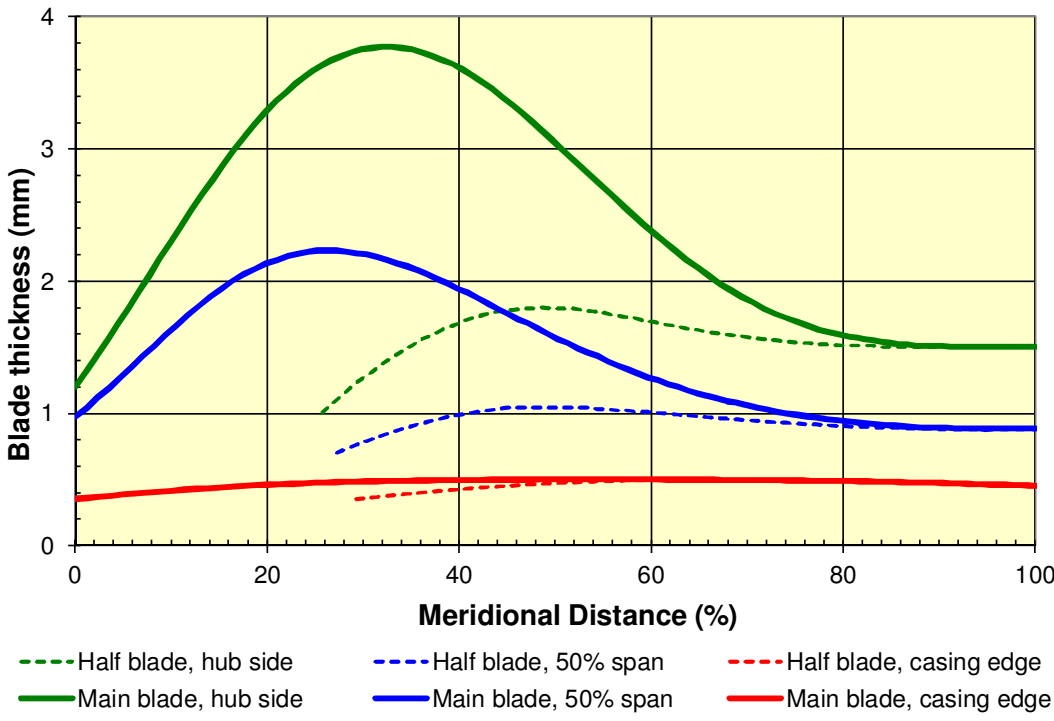

Fig. 3.2. Blade thickness distributions of the advanced compressor impeller with arbitrary surface

considered optimal trade-off between low end efficiency, surge margin and max flow capacity. Figure 3.1 highlights the difference between the advance compressor impeller and a

production

compressor impeller. Figure

3.2 and 3.3

illustrated the impeller blade thickness and blade angle distributions, respectively, of the advanced impeller design [35], along the meridional direction. The arbitrary

Compressor Blade (Beta) Angle Distribution

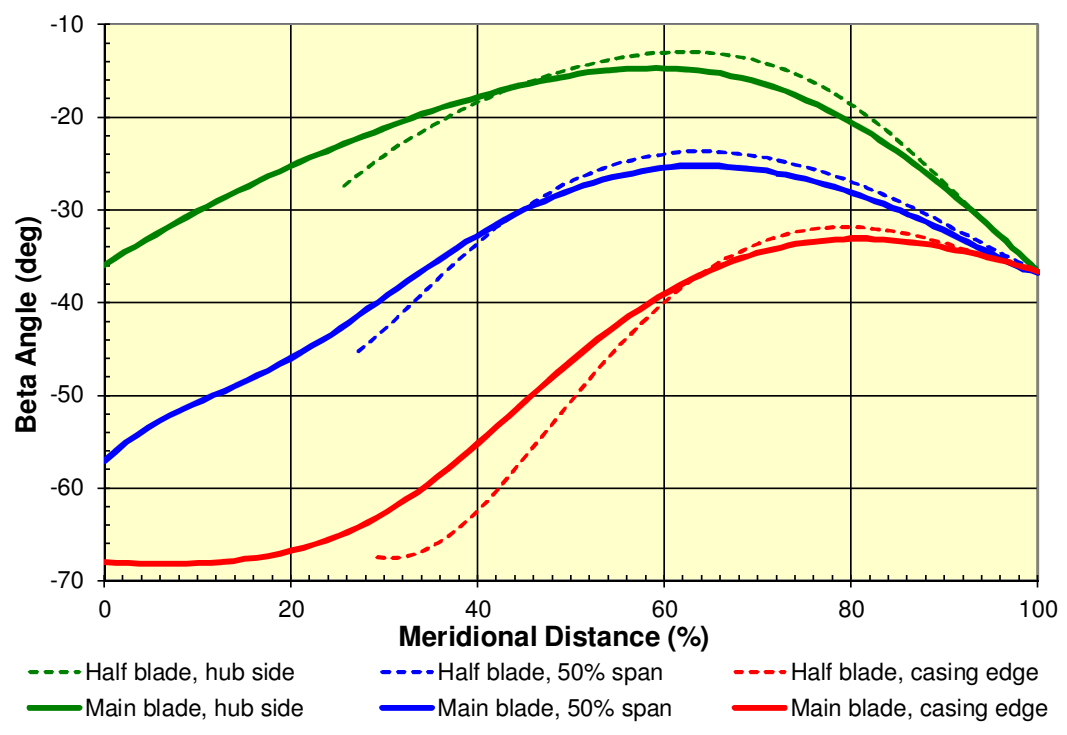

Fig. 3.3. Blade angle distributions of the advanced compressor impeller with arbitrary surface 
surface is represented with three sets of curves ranging from hub side, $50 \%$ span to shroud side. It is noticeable that the blade angle has more backward sweep at the impeller exit, compared to a conventional impeller design. The large back sweep angle at impeller exit typically offers better low end efficiency, at potential expense of pressure ratio and excessive blade stress. The blade angles at leading edges of full and split blade are also noticeably higher than conventional compressor impellers for automotive application, in order to improve compressor efficiency and surge margin at low mass flow area. The drawback of large blade angle at the leading edge is potential compromise of choke flow capacity.

In the 1D meanline design stage, the impeller blade passage throat area has to be sized to meet maximum flow capacity requirements.

Typically the blade passage throat area is geometrically represented by integration along the blade height of the shortest distance of the leading edge of the impeller to adjacent blades. It was well understood $[32,36]$ that this geometric blade passage throat area has to be further corrected for tip area blockage due to viscous effects, incidence loss, skin friction pressure loss, hub-to-shroud profile distortion, flow mixing and diffusion loss and impeller tip clearance leakage loss, etc. In other words, the geometric blade passage throat area has to be converted into an aerodynamic blade passage throat area which governs the impeller choking flow capacity.

It is a common practice to use one or two groups of splitter blades to optimize the trade-off between low end surge and efficiency requirements and high flow capacity requirements, i.e. to have increased blade passage throat area due to fewer blades at the compressor inlet. Besides the arbitrary surfaces, large beta angle at the impeller leading edge, the advanced impeller design is also featured by a relatively early introduction of splitter blades in the meridional direction, as illustrated by Fig. 3.2 and Fig. 3.3, compared to a conventional compressor impeller. Noticeably, the introduction of splitter blades creates extra geometric and aerodynamic blockages downstream of the impeller flow passage.

Constrained by capability of current meanline design software, casing treatment cannot be predicted thus optimized at 1D design stage and 3D design and CFD analyses have to be used for casing treatment design and optimization.

\subsection{Meanline design of the mixed flow turbine}

The mixed flow turbine is characterized by the non-radial inlet that reduces the flow path curvature and effectively reduces the secondary flow losses (Fig. 3.4). The other noticeable feature is that the radial flow turbine has the inducer blade angle parallel to the rotational axis while the mixed flow turbine typically has inducer blade angle round 
20-50 deg relative to rotational axis which helps the inducer to adapt to different instantaneous incidence angle of incoming exhaust flow that would vary with the flow rate and, more importantly, the pulsed pressure (Figure 3.5). Figure 3.6 and Figure 3.7

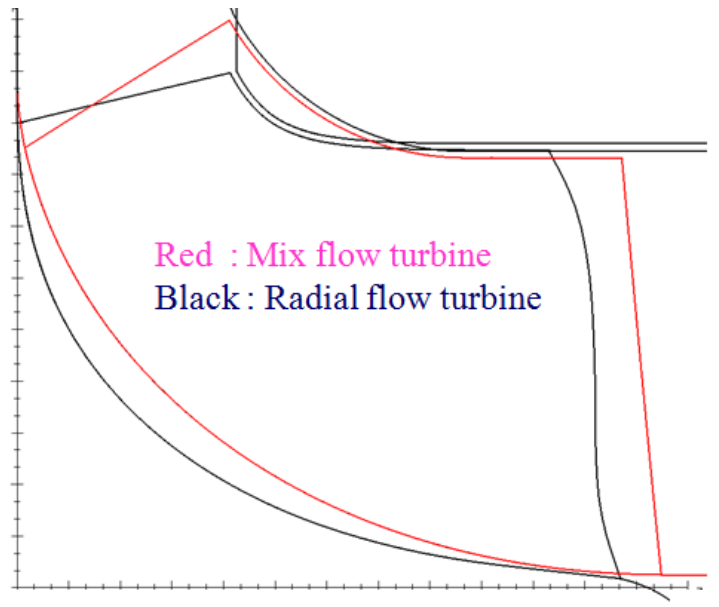

Fig. 3.4 Meridional flow passages of a radial flow (black) and a mixed flow turbine illustrate the blade thickness distribution and blade (beta) angle distribution of the advanced mixed flow turbine along meridional direction [35]. As shown in Fig. 3.4, the turbine inlet has a $15^{\circ}$ inclination angle, which makes it difficult to define the blade angle. The beta angle depicted in Fig. 3.7 is the angle between the turbine wheel rotational axis and the intersection line of extension of the blade onto a pseudo cylinder, with turbine inlet tip as its diameter and turbine wheel rotational axis as its center axis. Similar to a compressor impeller, the turbine wheel blade angle distributions are characterized with three locations at hub, $50 \%$ span and shroud side (100\%) span, respectively.

After meanline designs of compressor and mixed flow turbine completed, preliminary compressor and turbine performance maps would be available and GTPower engine simulation would be conducted and compared with program performance targets, in terms of full load torque, power as well as part load BSFC distributions under constraints of future feed gas emission requirements.

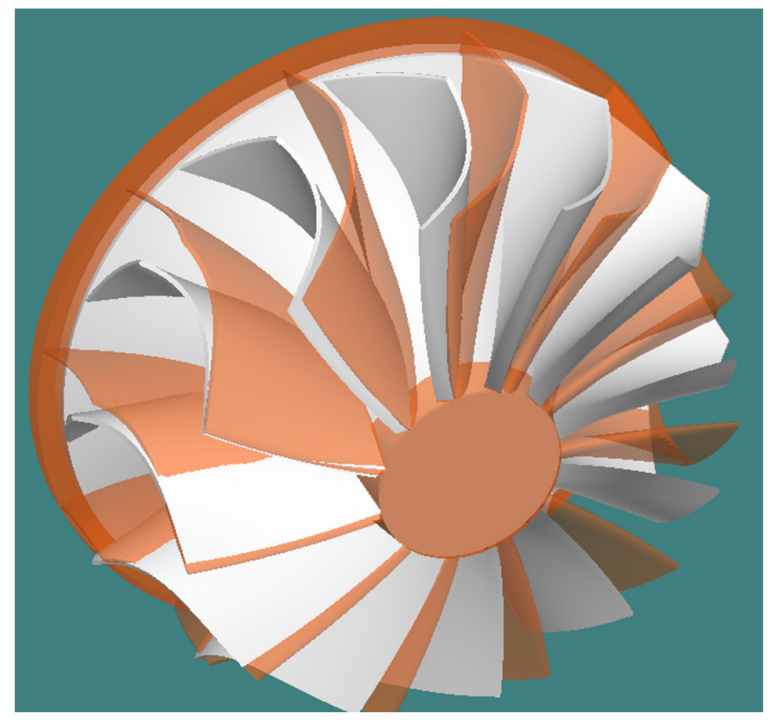

Fig. 3.5 Comparison of a conventional radial flow (red) and the advanced MD mixed flow turbine 


\section{Turbine Blade Thickness Distribution}

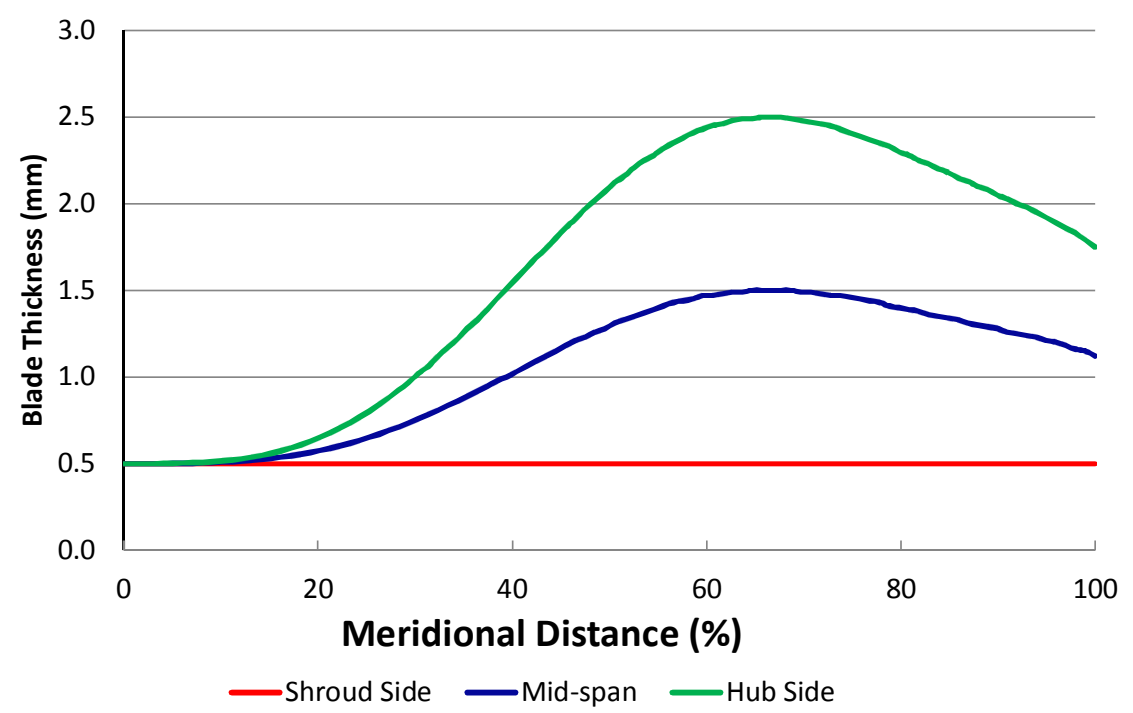

Fig. 3.6 Blade thickness distributions of the advanced MD mixed flow turbine [35]

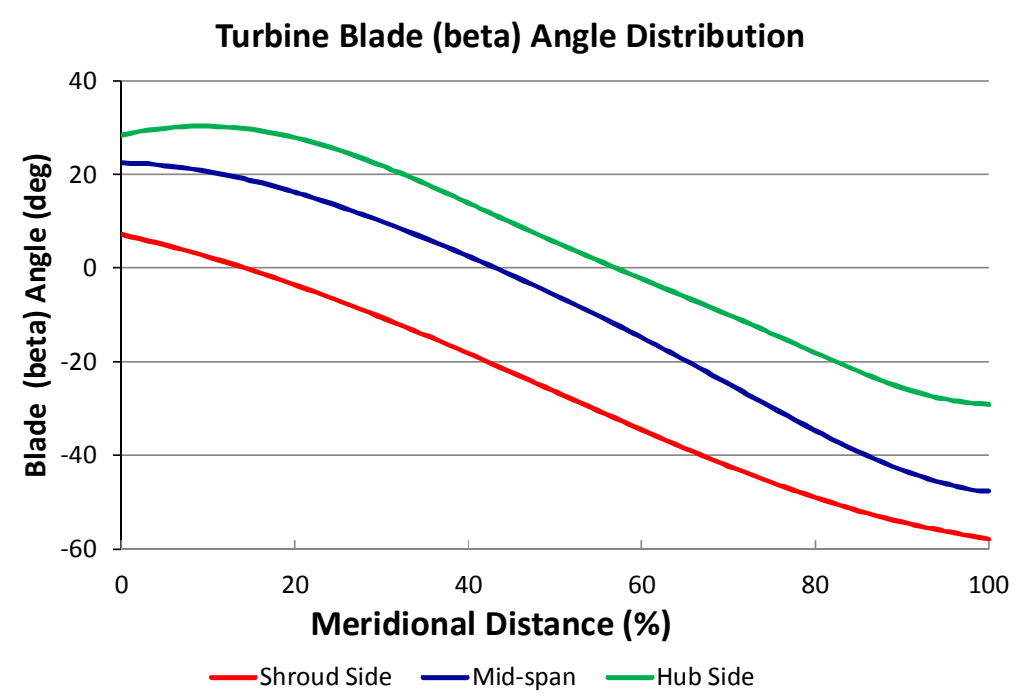

Fig. 3.7. Blade angle distributions of the advanced MD mixed flow turbine [35]

4. Three dimensional designs and analyses of turbocharger for performance validation and additional design iteration for structure and stress compliances 
The Compal and Rital software, meanline design tools for compressor and turbine design, respectively, are capable of predicting compressor and turbine performances. Similar to almost all meanline analytical tools, the Compal and Rital meanline software tools used in the meanline design stage have quite a few empirical correlations to deal with complex 3D physical mass transformation (e.g. flow separation, turbulent mixing, tip leakage, etc.) in 1D simulation, which are hardly universally applicable and may depend on the application and design geometry. To stretch the operation and design envelop of the turbocharger beyond what those empirical models were correlated, carefully validating the meanline design through 3D CFD is always prudent and necessary, even though 3D CFD may have its own challenges, especially in the compressor surge and choke areas.

Numeca Fine Turbo is extensively used in every design iteration between 1D and 3D design as a CFD simulation tool to validate the performance predictions out of meanline software Compal and Rital due to its effectiveness in turbomachinery analyses. The flow field visualization from Numeca is also a very useful resource to shed some light on the physics inside compressor and turbine and guide design changes to minimize flow losses and gain flow capacity. As always, we sometime have to trade time for accuracy, i.e. the 3D CFD is very time consuming and resource demanding. As such, we often use steady state single flow passage CFD, without volute, in design iteration stage to optimize blade design and active casing treatment.

Even though 3D CFD may be more accurate than meanline analyses, especially for the predictions of a concept geometry that no one have ever tested before, cautions must be taken in adoptions of turbulence model, wall function, and rotor-stator interfacing, etc. Therefore a model calibration is still needed. For that purpose, early on in the project, we took a compressor of which we have the geometry and detailed flow bench test data (i.e. pressure and temperature, mass flow between impeller exit and diffuser) and we conducted CFD model calibration.

\subsection{Compressor Optimization}

To meet the requirement of an advanced diesel engine with heavy EGR, an optimized centrifugal compressor with arbitrary blades and large inlet blade angle was developed. The blade was negatively bowed in the inducer area to control tip leakage flow. There are 6 main/splitter blades with vaneless diffusers. A conventional casing treatment (also called self-recirculation casing treatment or SRCT) was designed to extend the compressor surge flow while trying to minimize efficiency penalty, especially at low end. Figures 4.1 and 4.2 show the manufactured impeller and schematic of the SRCT, respectively. 


\subsubsection{Numerical Model for compressor CFD performance validation}

In present research, CFD was applied to guide compressor design, including optimization of impeller blades and SRCT. Commercial code EURANUS, integrated in Fine/Turbo interface, was used in the numerical simulation. It solves the time dependent Reynolds averaged N-S equations. The one equation turbulence model, Spalart-Allmaras model, was used in present simulation.

Onset of divergence or oscillation of numerical simulation may not accurately represent the onset of compressor instability, surge, or stall. However, the numerical instability is strongly related to the compressor unstable flow phenomenon. So for the purpose of relative comparison, the point when simulation became unstable was defined as onset of compressor instability.

\subsubsection{Modular compressor design and simulation}

Replaceable inserts of the compressor shroud were designed so that performance of the compressor, with and without SRCT (Fig. 4.2), could be tested and evaluated separately. Compressor performance was tested on a steady state turbocharger flow bench. After the compressor without casing treatment was tested, casing treatment inserts were replaced and the same test was performed to study the effect of SRCT's effect on compressor performance.

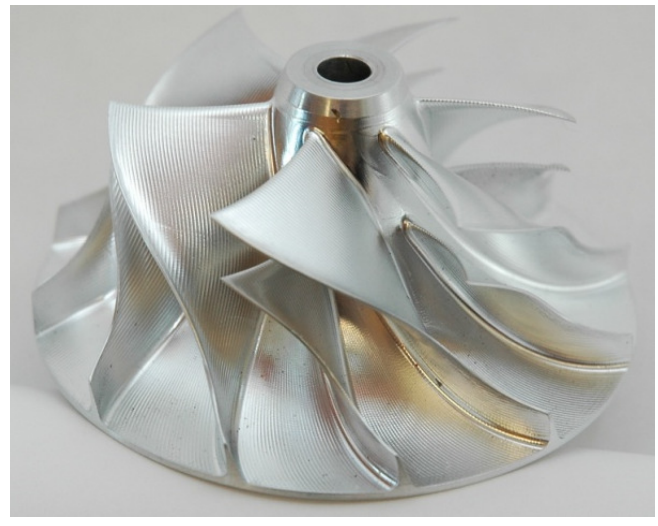

Fig. 4.1 Centrifugal Impeller

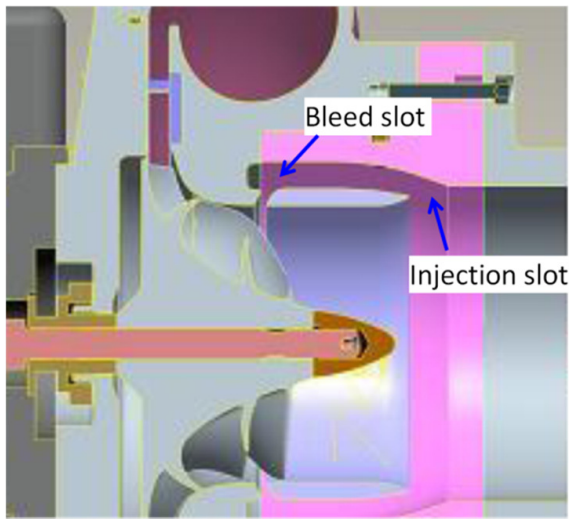

Fig. 4.2 Conventional self-recirculation casing treatment

4.3 Numerical investigation of SRCT impact on performance 


\subsubsection{Casing treatment impact on surge}

Figure 4.3 is a comparison of measured and simulated compressor performance with and without casing treatment. Simulation data shows that compressor surge has been suppressed with SRCT at medium and high rotation speed without visible efficiency penalty from peak efficiency to surge condition. At low turbo rotation speed, there is no visible surge margin improvement with SRCT. However, there is some performance penalty near choke condition at all turbo rotation speeds.

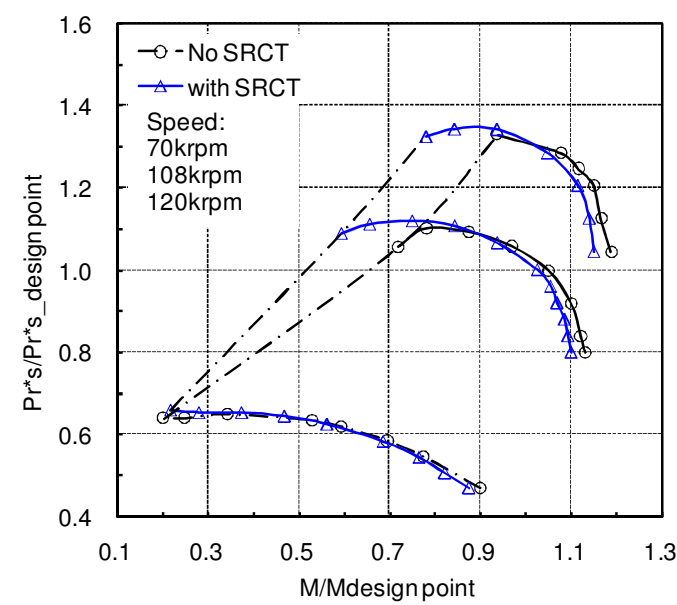

Simulations

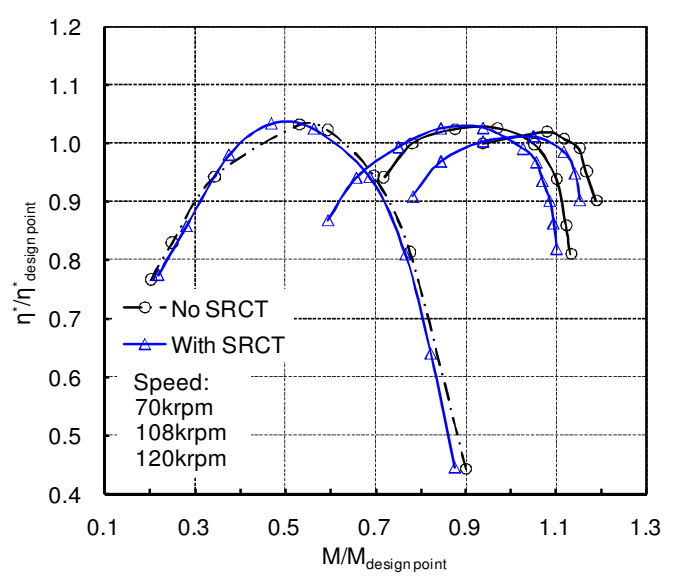

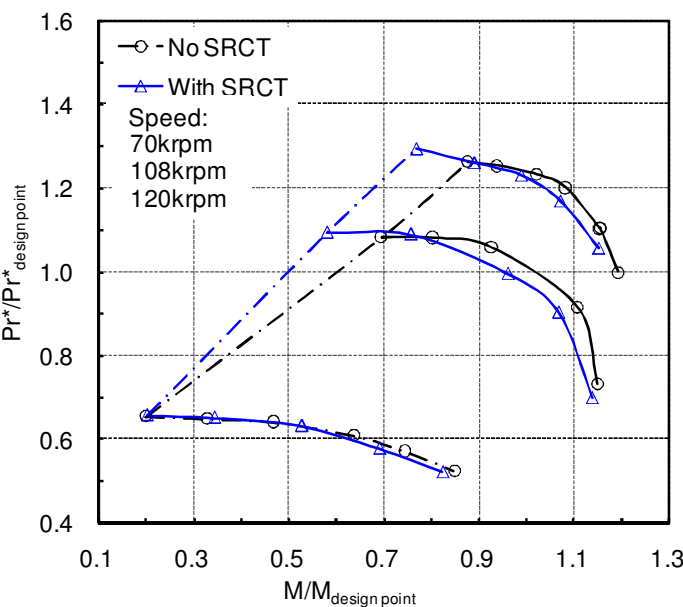

Measurements

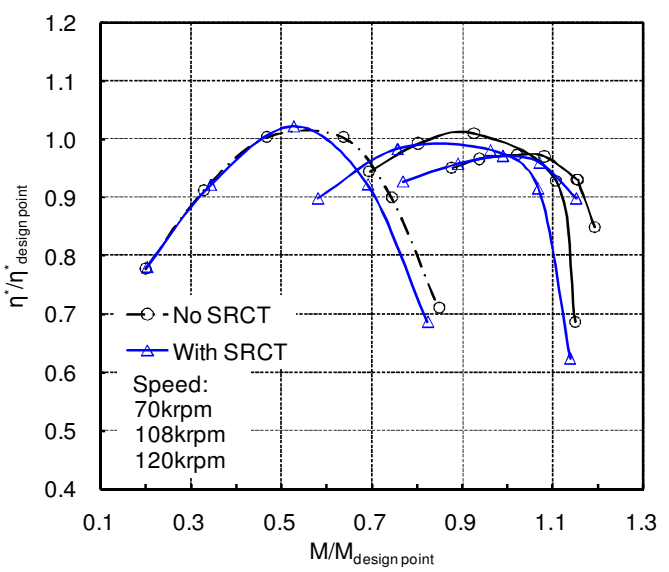

Fig. 4.3 Comparison of Measured and Tested Compressor Performance with and without SRCT

Amelioration of surge margin was calculated according to the following formula: 


$$
\Delta \varphi=\left(\mathrm{M}_{\mathrm{smooth}}-\mathrm{M}_{\mathrm{SRCT}}\right) / \mathrm{M}_{\text {smooth }}
$$

$\mathrm{M}_{\mathrm{SRCT}}$ and $\mathrm{M}_{\mathrm{smooth}}$ are surge flow of the compressor with and without SRCT, respectively.

The measured data shows that SRCT extended surge margin of the compressor at medium and high rotation speeds. With SRCT, there is about $20 \%$ surge margin improvement without efficiency penalty from the peak efficiency point to near surge condition on each speed line, which is consistent with the numerical simulation. However, near choke condition there is about $2-3 \%$ mass flow rate reduction due to SRCT, which also supported the CFD result. The compressor's overall flow range, efficiency, and pressure ratio predicted by CFD also agree well with test data. The CFD analysis tool we used could predict the SRCT's effect on compressor performance very well. Therefore it was extensively utilized to optimize SRCT to improve compressor performance.

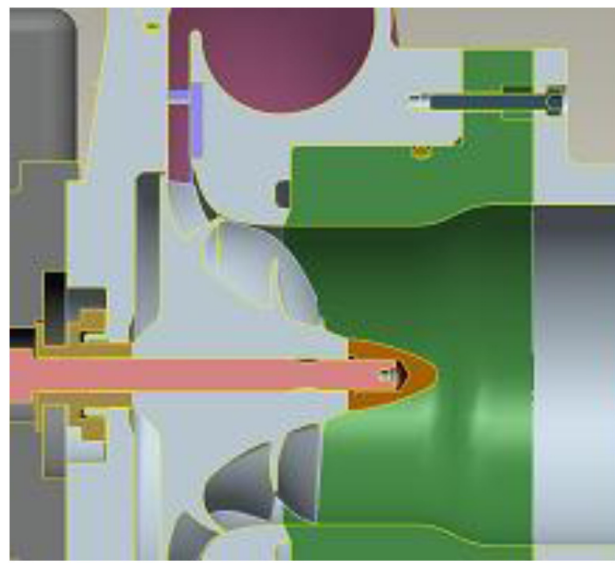

Fig. 4.4a: no casing treatment

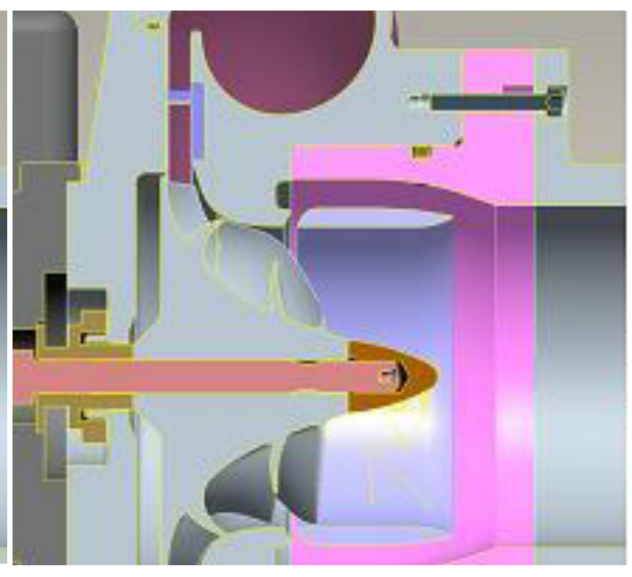

Fig. 4.4b: casing treatment $A$ 


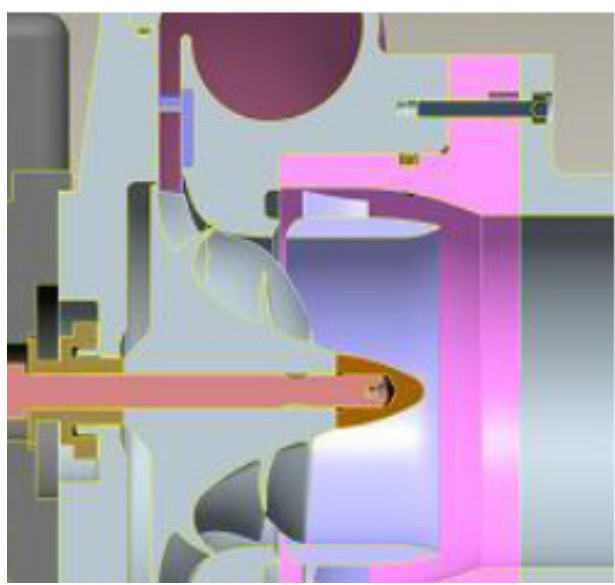

Fig. 4.4c: casing treatment $B$

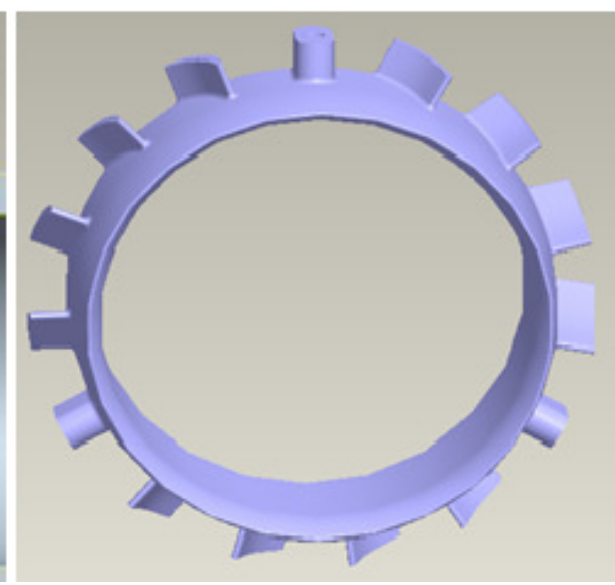

Fig. 4.4d: guide vanes in casing treatment $B$

Fig. 4.4. Three casing treatment configurations are investigated: base (Fig.2a: no casing treatment); casing treatment A (Fig. 2b); casing treatment B (Fig. 2c: with annular guide vanes inside the casing treatment). [43, 44]

To further investigate the effects of recirculating casing treatment interactions, a set of hardware was designed and fabricated for bench testing and experimental validation.

For convenience and consistency in bench testing, a modular compressor housing was designed and used, in which the casing treatment inserts can be switched to construct different casing treatment configurations. Figure 4.4 shows three compressor casing treatment configurations that have been investigated numerically and experimentally to quantify the benefits of a conventional single-port casing treatment (Fig. 4.4b, casing treatment A) and a casing treatment with air foil guide vanes inside (Fig. 4.4c, casing treatment $B$, which is similar to casing treatment $A$ except that there are guide vanes inside the casing treatment) over the base design that does not have a casing treatment at all (Fig. 4.4a). The slot was sized and located with a focus on improving the surge margin of the stage with less emphasis placed on increasing flow capacity. 
The various casing treatment arrangements were evaluated numerically using Numeca's RANS CFD code, FINE/Turbo (Refer to [43, 44] for details). Figure 3 provides a comparison of CFD predicted relative Mach number contours at near choke flow conditions for the "no casing treatment" and "casing treatment option A" (no guide vanes inside casing treatment) configurations. The numerical simulation confirms that for the near choke flow conditions, injection of extra bypass air from the single injection slot before splitters is not likely to significantly alter the flow capacity of the stage. The blade passages were mostly occupied by shock wave on both sides of leading edge of the splitter blades, with and without casing treatment, i.e., the leading edge of the splitter blades is where the aerodynamic throat is, and introduction of additional air through casing treatment before this throat does not help to mitigate the choke flow. As a matter of fact, the CFD analysis showed that the air injection before the splitter blades has slight penalty in choke flow capacity $[43,44]$ for this particular compressor design.

These various casing treatment arrangements were further tested at a steady state flow bench at ConceptsNREC. Figure 4 illustrates compressor efficiency and pressure ratio vs. mass flow at different wheel speeds based on flow bench test data of the modular compressor with different casing treatment arrangements. During the flow bench test, on each speed line the operating point at low mass flow area would stop when surge (substantial oscillation in mass flow and pressure measurements) occurred. The test data corroborates the numerical simulation with both casing treatments variants (with and without guiding vanes in the recirculation path), showing significantly enhanced surge margin without efficiency penalty (Fig. 4.4a). Unfortunately, the pressure ratios at high flow rates have been compromised with the casing treatments $A$ and $B$ (Fig. 6b). It is obvious from the steady state flow bench test that the introduction of additional air before the splitter blade, or before the aerodynamic blade passage throat, may adversely impact the compressor choke flow capacity.

Consistent with the study by Sakaguichi [45] and our flow bench test data (Fig. 7.1), our CFD simulation [43,44] also visualized that the air foil guide vanes inside the casing treatment (option $\mathrm{B}$ ) slow down the rotation of air inside the recirculation slot and adjust the flow incidence angle when mixing with the mainstream, thus suppressing the circumferential component of the air injection velocity and improving flow stability in the impellers near stall or surge conditions. It seems that the extension of the stability zone by guide vanes inside casing treatment (option B) is more effective at higher pressure ratio.

\subsubsection{Compressor CFD flow visualization and flow field analysis}

To investigate how the casing treatment affects compressor performance, the internal flow field with and without casing treatment was analyzed and compared. 
Figure 4.5 is a comparison of relative Mach number on compressor shroud side near choke condition. Without SRCT, there is transonic flow downstream of the leading edge of main blade on the suction side. Near the leading edge of splitter blade, there is strong supersonic flow on both the suction and pressure sides and the peak Mach number area is located downstream of the splitter leading edge. Downstream of the flow passage, there is some area with low momentum, which is due to accumulation of the tip leakage flow. With SRCT, calculation of mass flow showed there is about $11 \%$ of stage mass flow injecting into the impeller passage from the SRCT with current impeller design. As a result, the mass flow through the impeller inlet has been reduced and the supersonic flow in the upstream of the bleed slot has been reduced. However, the maximum flow capacity is still limited by the passage throat near splitter blades' leading edge. As the air injecting into impeller passage from SRCT bleed slot, choke happens near the throat and it even resulted in flow capacity penalty due to mixing blockage compared to the one without SRCT.

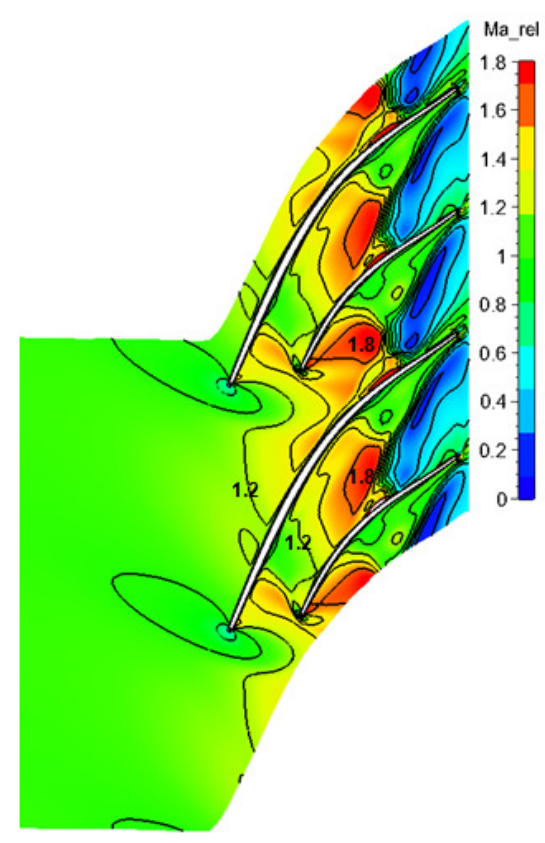

(a) Smooth Wall

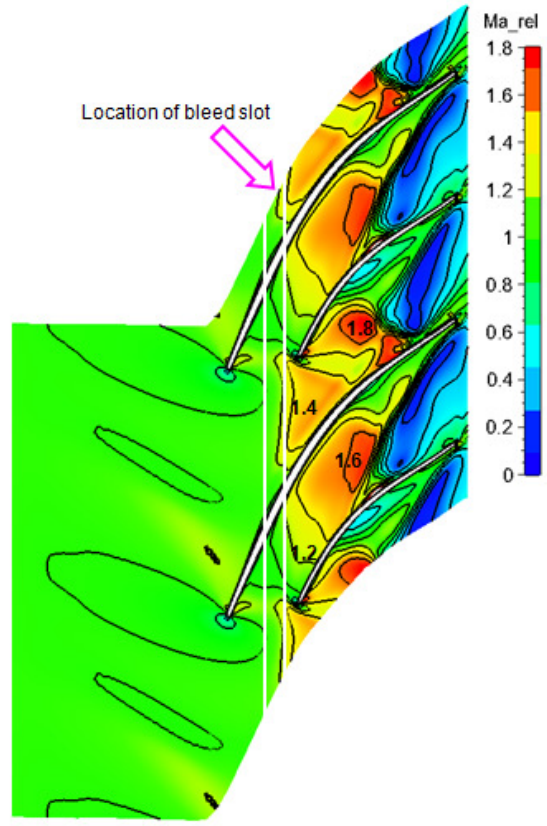

(b) With SRCT

Fig. 4.5 Relative Mach Number Distribution Near Choke Condition

Figure 4.6 shows interaction of tip leakage with main flow near surge condition of the compressor with and without SRCT. The relative Mach number distribution shows 
that without SRCT, there are shock waves on the suction sides of the main impeller blades leading edge. The relative Mach number before the shock wave is about 1.4. Between the leading edge of the main and splitter blade, there is an interface between the low and high momentum fluid, which is due to interaction of tip leakage with main flow. As illustrated in Fig.4.6 (e) [37], there is strong tip leakage over the blade that has the tendency to flow back towards the impeller inlet due to negative pressure gradient. The reverse flow will interact with the incoming main flow. As shown in Fig. 4.6 (a), the interaction surface starts from suction side of the main blade and extends towards pressure side of the adjacent main blade. This interaction of tip leakage with main flow is the main reason for the onset of compressor stall as reported in reference [37, 38, and 41]. Downstream of the interaction surface, the fluid has negative velocity as shown in Figure 4.6 (c), which is mainly due to tip leakage recirculation flow. With SRCT, the inlet relative Mach number is reduced and there is no shock wave on the suction side of the main blade near the leading edge. As explained by Hunziker [42], it's mainly due to the negative pre-swirl added to the incoming main flow by the recirculation flow which has been propelled by the impeller. The interaction surface of tip leakage with main flow, which exists in the compressor with smooth wall, initiated from suction side of the main blade, as illustrated in Fig. 4.6 (a) beginning from the suction surface of the main blade, disappears upstream of and near the area that is covered by SRCT. There is no low momentum fluid caused by the tip leakage in that area, nor the fluid with negative velocity. But downstream of the bleed slot, there is some low momentum fluid accumulated that has negative axial velocity. Obviously, the tip leakage flows back towards the impeller inlet inside the impeller passage near shroud before it is "sucked" into the recirculation cavity. In that case, its interaction with the shock wave near the impeller leading edge could be suppressed (Fig. 4.6 (a) and Fig. 4.6 (b)). Therefore, with SRCT, the impeller inlet Mach number is reduced and so is the interaction of tip leakage with shock wave near impeller inlet, both of which contribute to suppression of compressor surge.

From the above analysis it can be seen that with the current design, SRCT can't extend compressor choke flow while on the contrary it leads to some flow capacity penalty near choke condition. One simple remedy is that if the bleed slot was moved downstream of passage throat where the minimum pressure area is located, the pressure difference between the SRCT cavity and area downstream of flow passage throat could be increased and more flow could be by-passed into the passage through the SRCT cavity. The by-passed flow won't be limited by the impeller throat anymore and the SRCT may extend compressor flow capacity. Fig. 4.6 shows that most choked area is just behind the leading edge of the splitter blade and if the SRCT bleed slot is placed in that area, it may increase compressor choke flow rate. However, another issue will arise: The recirculation flow near surge condition will increase due to 
increased pressure differential between the bleed slot and compressor inlet, which leads to compressor efficiency deterioration. To reduce the recirculation flow loss and the compressor efficiency penalty at low end, the bleed slot needs to be placed upstream like conventional SRCT design. It is necessary and possible to design an active SRCT, i.e. two bleed slots need to be designed for the compressor SRCT: One is for choke control, named choke slot located downstream of the splitter blades' leading edge to extend choke flow; the other one is for surge control, named surge slot, located upstream of the choke slot to improve the compressor efficiency near surge condition. Based on the above concept, numerical analysis of the choke and surge slots with different configurations was carried out.

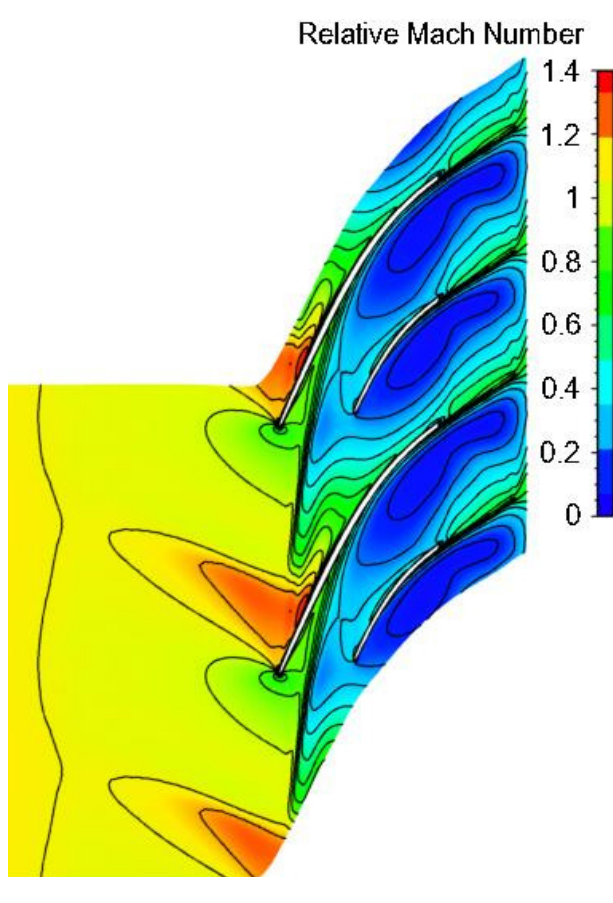

(a)

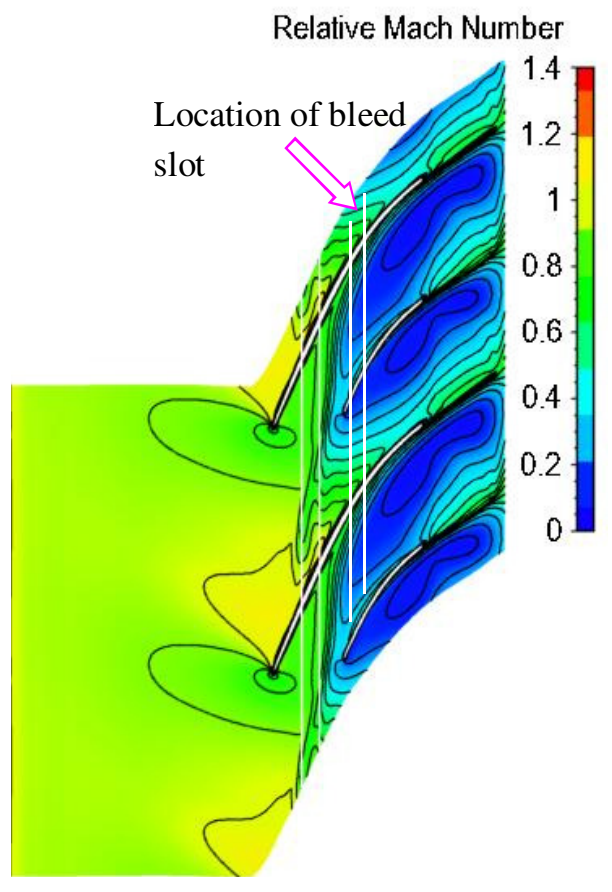

(b) 


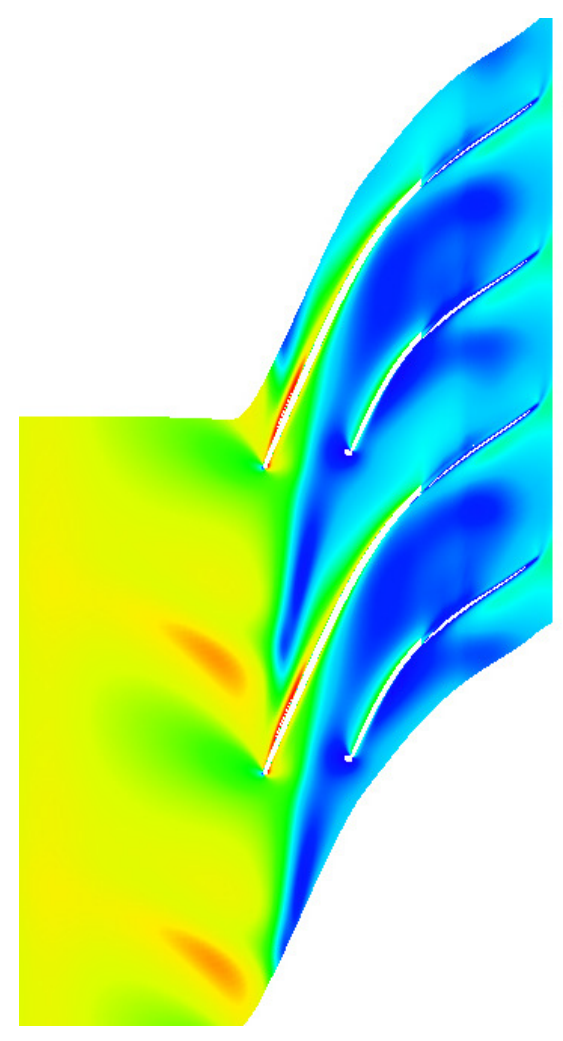

(c)

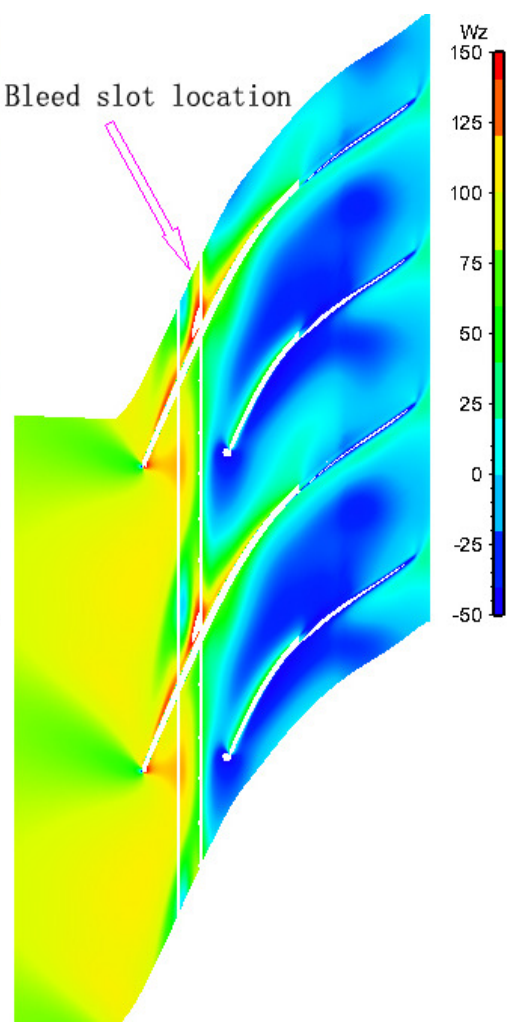

(d)

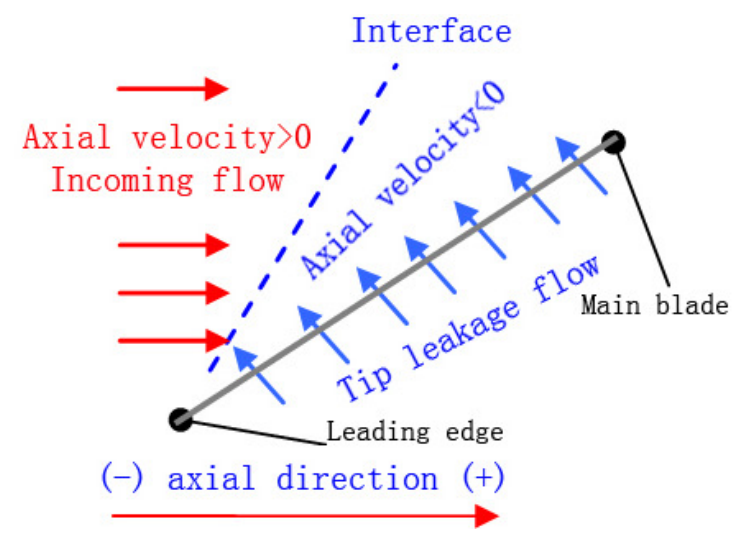

(e)

(a) Ma_rel distribution without SRCT (b) Ma_rel distribution With SRCT (c) Axial velocity distribution without SRCT (d) Axial velocity distribution with SRCT (e) Illustration of interaction of main flow with tip leakage

Fig. 4.6 Interaction of tip leakage with main flow near surge 


\subsection{Optimization of choke slot for operation range extension}

As current design was trying to extend maximum flow capacity of the compressor which determines maximum power of engine, optimization of bleed slot was carried out at the compressor's maximum operating speed of $135 \mathrm{krpm}$. Three parameters; location, lean angle, and width of the bleed slot, were investigated in present research. The static pressure difference between the compressor inlet and bleed slot is the main force to drive the flow, which is primarily determined by location of the bleed slot. First, different locations of bleed slot were investigated. Fig. 4.7 shows azimuthally averaged static pressure near choke condition of the compressor without SRCT. It can be seen that the minimum static pressure located downstream of the splitter blade leading edge could be chosen as the location of bleed slot to extend choke flow rate. Three different locations of the bleed slot, choke case 1, 2, and 3, as shown in Fig. 4.7, were numerically studied. All of the three cases have a bleed slot width of $2 \mathrm{~mm}$. The center of the bleed slot in case 2 is located in the minimum pressure area and in the other two cases the bleed slots were moved upstream and downstream $3 \mathrm{~mm}$ separately compared to case 2 . After the location of the bleed slots were optimized, lean angle and width of the bleed slots were numerically studied. 

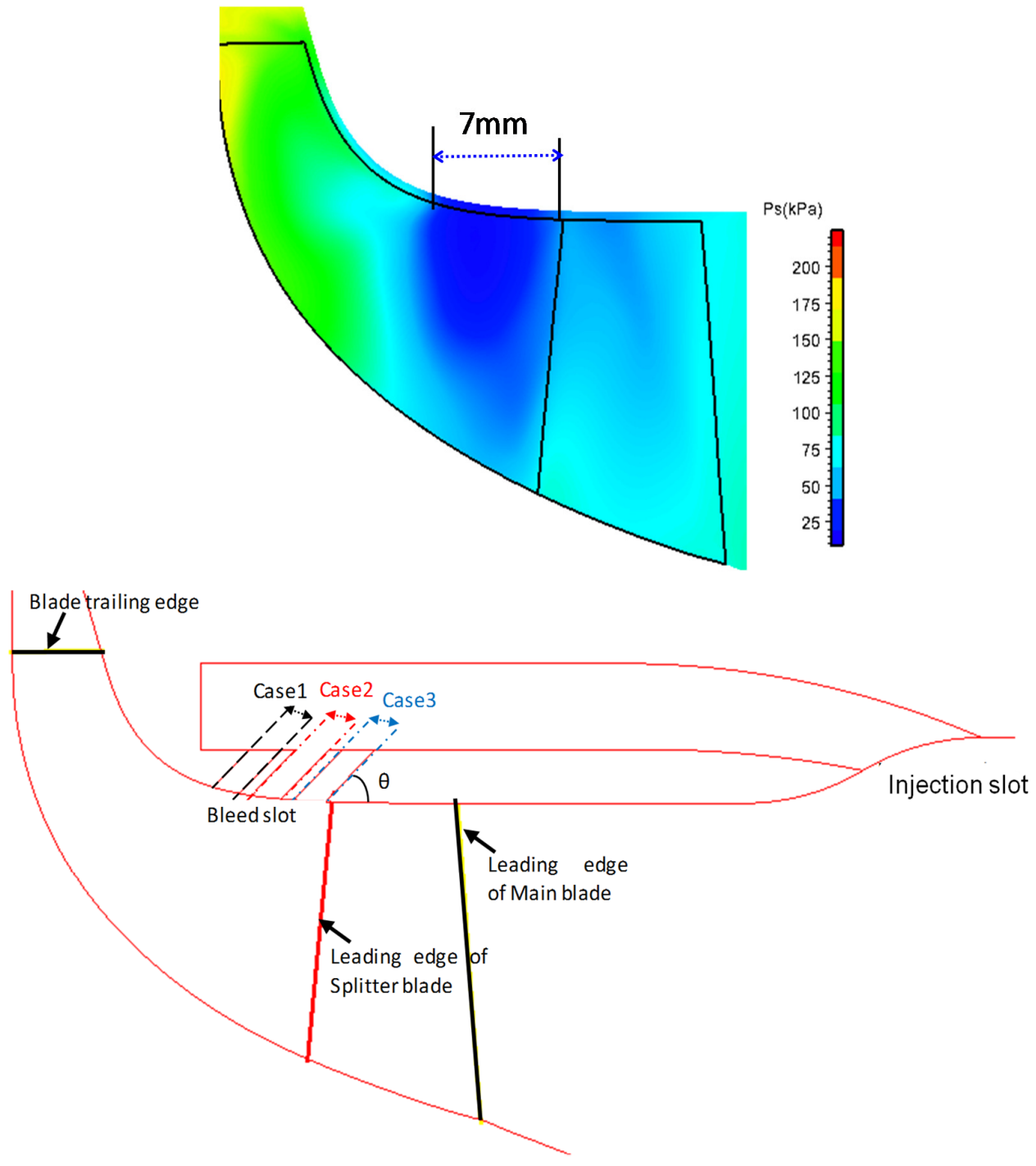

(a) Azimuthally Averaged Static Pressure (b) Configurations with Different Bleed Slot Location

Fig. 4.7 Azimuthally averaged static pressure and location of bleed slot

Figure 4.8 shows compressor performance comparison with different SRCT bleed slot locations. It can be seen that bleed slot location has a great effect on 
compressor performance. With all the 3 simulated SRCTs, the choke flow rate has been extended compared to the compressor without SRCT. The compressor choke flow rate with choke case 1 and 2 is larger than the one with choke case 3 . In the extended choke flow area, the compressor maintains the same or even better efficiency compared to the one without SRCT. As the compressor operating condition moves to lower mass flow rate, the compressor with choke case 1 has much lower efficiency and pressure ratio, while the compressor with choke case 3 shows the best efficiency and pressure ratio among the three cases. This phenomenon is related to the pressure difference between compressor inlet and the bleed slot and the substantial recirculation flow through the SRCT. Figure 4.9 shows the recirculation flow rate through the SRCT cavity. With choke case 1, there is more recirculation flow through the SRCT than the other two cases, which will create more flow loss inside the SRCT and more mixing loss when it injects into the main flow through the upstream slot. As a result, the compressor efficiency is the lowest. Including the work done by the impeller and negative swirl added, when the recirculation flow mixes with the main flow, negative swirl has been carried over. According to the Euler equation, the head rise will be reduced. For the bleed slot located more downstream in the impeller passage, the recirculation flow will contain more negative swirl added by the impeller and it will have more impact when mixing with the incoming main flow. As a result, the compressor with choke case 1 shows the lowest pressure ratio. With choke case 3 , the recirculation flow through the SRCT has been reduced. Therefore, the compressor with choke case 3 shows the best efficiency among the three cases. There is only a little peak efficiency penalty and it remains the same efficiency level, compared to the case with smooth wall near surge condition. With choke case 3, the compressor shows the highest pressure ratio, which is also due to the reduction of negative swirl added by the recirculation flow. The choke case 3 is able to extend both the choke and surge flow nearly without efficiency penalty. It shows better performance than the previous SRCT design. Although the compressor with choke case 2 shows lower efficiency than the one with choke case 3 in the low flow area, we can count on the separate surge slot located upstream to improve compressor performance at low end. So choke slot location was chosen as case 2 to get maximum flow capacity. Then lean angle and width effect of the bleed slot were studied at this location. 

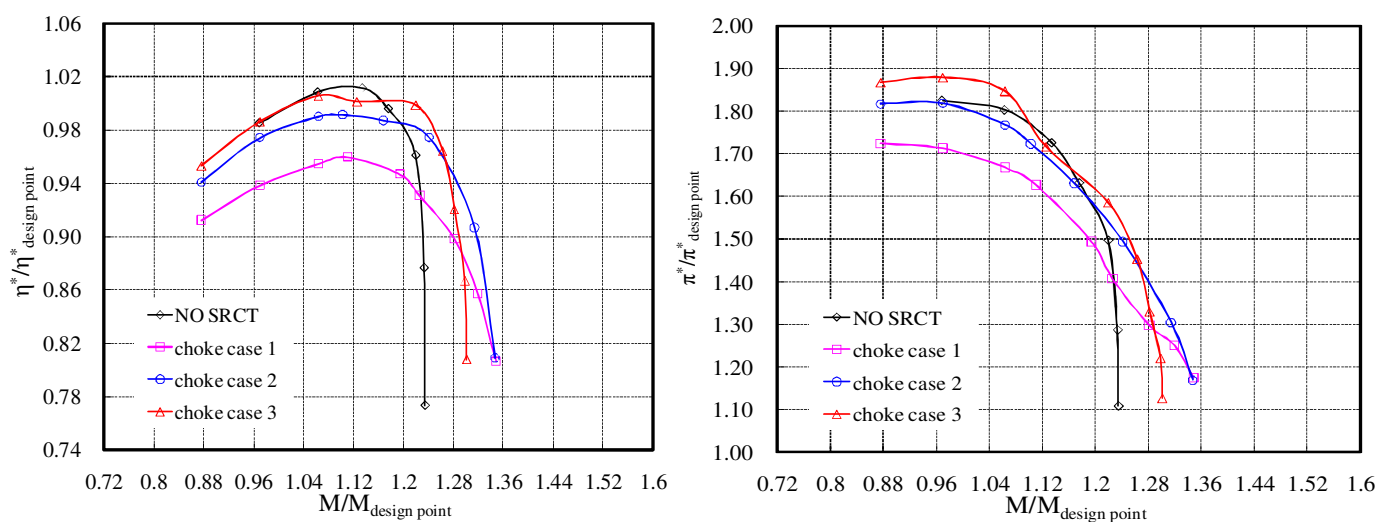

Fig. 4.8 Compressor Performances with Different SRCT Bleed Slot Location

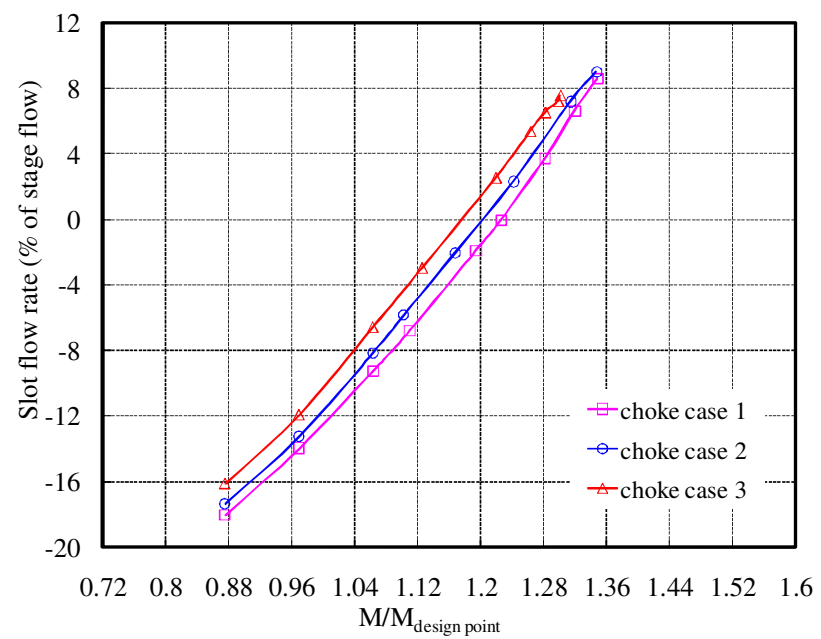

Fig. 4.9 Recirculation flow rate with different SRCT bleed slot location

Figure 4.10 is a performance comparison of the compressor with different bleed slot lean angles. The angle between the bleed slot and the impeller ration axis was defined as the lean angle $\theta$, as shown in Fig. 4.7. In the simulated cases with different lean angles, the slot width in the axial direction all remains $2 \mathrm{~mm}$ in the different lean angle configurations. From Fig. 4.10 it can be seen that the lean angle also has some effect on the compressor performance, especially near choke condition. But it is far less sensitive compared to the slot location's effect. With $90^{\circ}$ angle, the efficiency is the 
lowest among all the configurations near choke condition. It is mainly due to the fact that near choke condition the by-passed air flows almost perpendicularly from the bleed slot into the impeller passage in the $90^{\circ}$ configuration, which will lead to more flow loss when injection air mixing with the main flow. As the slot width remains $2 \mathrm{~mm}$, with reduced lean angle, the effective flow width will be reduced and so will the bypassed flow rate, which will contribute less to the performance improvement. The compressor with $60^{\circ}$ shows best performance in the extended choke flow rate. So the lean angle of the choke slot was chosen as $60{ }^{\circ}$ and slot width was optimized at this angle.
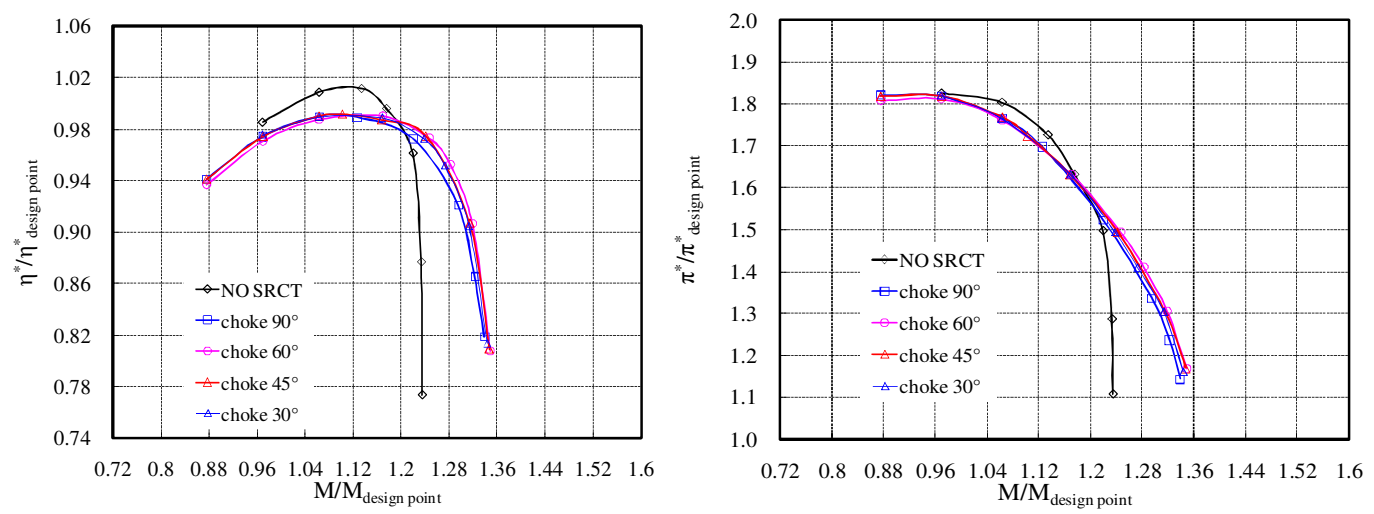

Fig. 4.10 Compressor performance with different SRCT bleeding slot lean angle

Figure 4.11 is the performance comparison of the compressor with different bleed slot widths. For different bleed slot width, the center line of the slot remains the same as that in the choke case 2 as shown in Fig. 4.8. From Fig. 4.12 it can be seen that the bleed slot width significantly affects the compressor performance. With bleed slot width of $2 \mathrm{~mm}$, the choke flow rate of the compressor has been increased about $9.5 \%$ compared to the compressor without SRCT. The choke flow rate of the compressor rises gradually with further increase of the slot width. With $6 \mathrm{~mm}$ bleed slot, there is about $15 \%$ increase in compressor maximum flow capacity. When the slot width is further increased, the growth of the choke flow is much slower. From Fig. 4.8 it can be seen that the low pressure area is about $7 \mathrm{~mm}$ and, with about $6 \mathrm{~mm}$ slot width, the slot almost covers the entire low pressure area. Obviously, when the bleed slot is as wide as the low pressure area, it could make full use of the pressure difference between the upstream and bleed slot, and thus maximum flow capacity extension could be obtained. 
The compressor efficiency in the extended choke area is also gradually improved with increase of the slot width; however, deteriorations in the compressor low-end efficiency and pressure ratio are not acceptable.
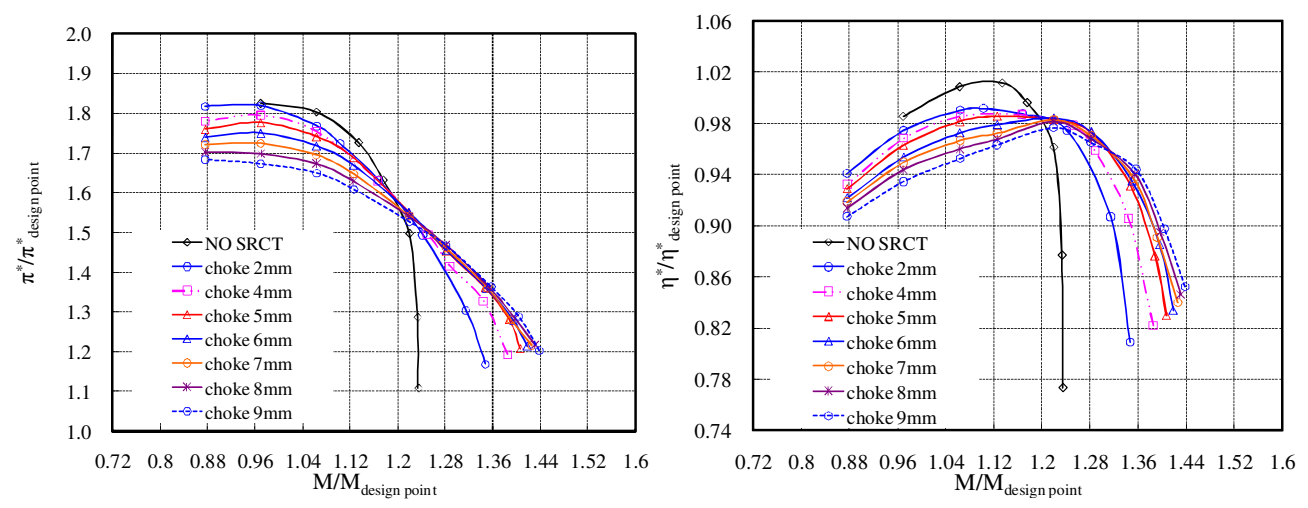

4.11 Compressor performance comparison with different bleeding slot width

Fig. 4.12 is the comparison of mass flow rate through the SRCT cavity with different bleed slot width. Near choke condition, the bypassed flow into the impeller passage increases with the slot width, consistent with the changes in the compressor choke flow rate with different slot width. Near surge condition the recirculation flow rate, as well as the stage efficiency penalty, initially increases with the slot width; however, when the slot width is wider than $5 \mathrm{~mm}$, the recirculation flow will decrease. Flow-field investigation shows that when the slot width is wider than $5 \mathrm{~mm}$, there is strong separation flow in the bleed slot. That means that wider bleed slot, even with less recirculation flow rate, leads to more recirculation loss, thus larger efficiency penalty. To improve the low end compressor performance, a surge slot located upstream of the choke slot is needed and will be investigated in the next section. 


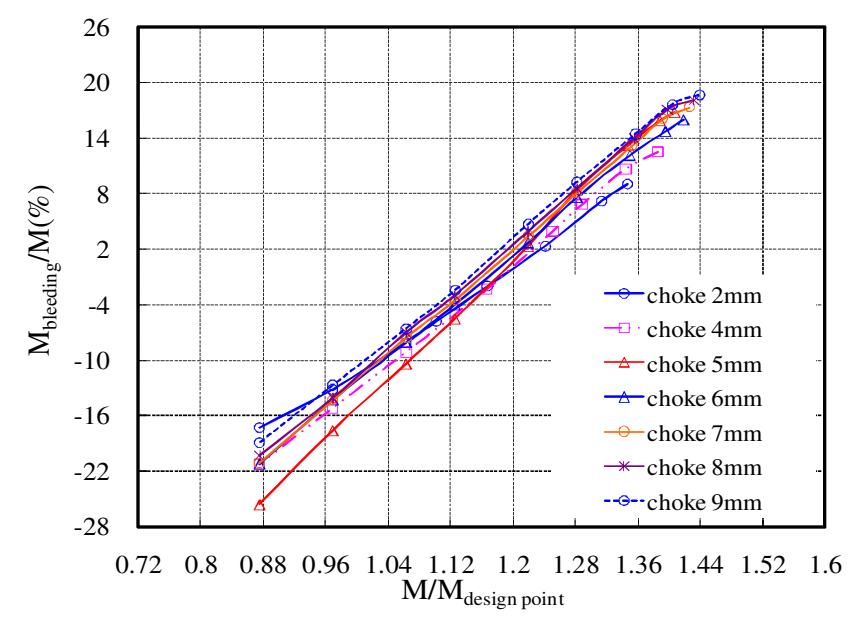

Fig. 4.12 Recirculation flow rate with different width of SRCT bleed slot

Figure 4.13 shows the relative Mach number comparison of the compressor with and without SRCT near choke condition at shroud side. Without SRCT, there is strong shock wave before the leading edge of the main blade. The shock wave extends to the adjacent main blade and strong supersonic flow exists on the suction side of the main blade. The splitter blade leads flow passage reduction and strong supersonic flow is formed, which is choke phenomenon in passage throat and limits compressor maximum flow capacity. In downstream of the passage throat, some low momentum fluid is accumulated, mainly due the tip leakage from pressure side to suction side. With SRCT, the choked area has been moved to the downstream of the bleed slot. It's due to the fact that the actual flow through the passage throat has been reduced and the by-passed air leads choke of the area downstream of the bleed slot. Thus the shock wave and supersonic flow upstream of the bleed slot have been shrunk significantly. Besides that, injection of by-passed flow can suppress the tip leakage and the accumulation of low momentum fluid in downstream of impeller passage has been great improved. Both the weakened shock wave upstream of the bleed slot and the tip leakage downstream of the slot help to improve compressor performance. As a result, even with more bypassed flow through the bleeding slot and potentially mixing loss associated with it, there is nearly no efficiency penalty with the wide bleed slot near compressor choke condition. 
With the optimized choke bleed slot, although the flow capacity of the compressor has been greatly extended, there are about 4-5\% efficiency penalty at low end, which indicates that a separate surge slot is needed to improve compressor efficiency in that operation range.
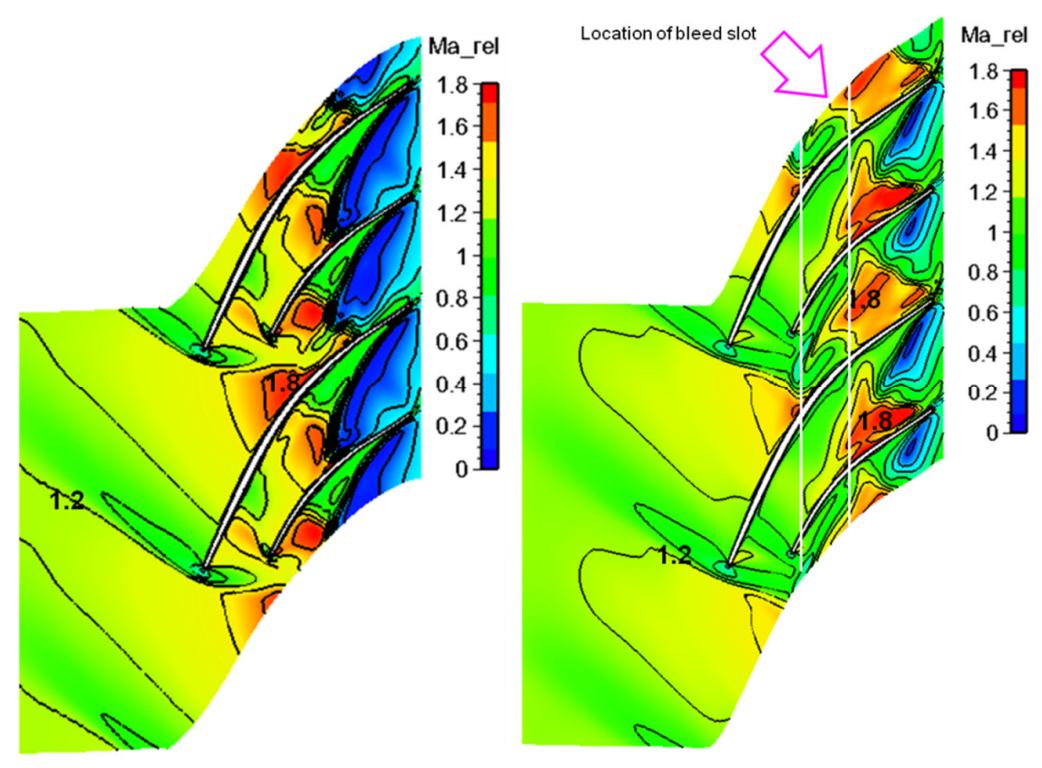

(a) Without SRCT

(b) with SRCT Choke Slot, Bleed Slot Width $=6 \mathrm{~mm}$

Figure 4.13 Relative Mach number comparisons of compressor at choke with and without SRCT near shroud

4.5 Optimization of surge slot for optimal low end efficiency and surge margin

From the above analysis it can be seen that with choke slot open, there is a lot of efficiency penalty at low end. To improve the efficiency at low end, another bleed slot, named surge slot for surge control in current research, is proposed. The surge slot is located upstream of the choke slot. By moving the bleed slot upstream, the pressure difference between the bleed slot and compressor inlet will be reduced, and so is the recirculation flow through the SRCT and flow loss near compressor surge condition. However, the circulation flow has to be maintained above reasonable rate so that 
bleeding of the tip leakage and negative pre-swirl added to the main flow is able to suppress the interaction of the tip leakage with the shock wave and extend compressor surge margin effectively.

Based on the previous simulation of the configurations with different choke slot lean angle, the same angle of 60 was chosen for the surge slot. Then three different bleed slot locations between the main and splitter impeller leading edge were investigated. They were $1 \mathrm{~mm}$ in axial distance, as illustrated in Fig. 4.14. Different widths of bleed slot were also numerically investigated at bleed slot location as labeled "Case 1". As compressor surge is a system phenomenon which includes effect of the volute; therefore simulation for the separate surge slot was modeled with a volute. Figure 4.15 shows the compressor performance comparison with different separate surge slot configurations. For comparison, the compressor performance with optimized choke slot is also given. It can be seen that the location of the bleed slot also affects the compressor surge flow. When the bleed slot is too close to the leading edge of the main blade as in case 2 and 3, the SRCT is less effective to extend compressor surge flow than in case 1. It's mainly due to the fact that the amount of recirculation flow has been reduced when the bleed slot is too close to the impeller leading edge, and it won't be able to suppress the interaction of tip leakage flow with the shock wave. In fact, the bleed location at case 1 has just been moved $1 \mathrm{~mm}$ upstream relative to the conventional single design. When the slot width is increased from 2 to $3 \mathrm{~mm}$, there is slight efficiency penalty near surge, which is due to increased recirculation flow loss. Finally, the surge slot was chosen as case 1 with $2 \mathrm{~mm}$ in width. 


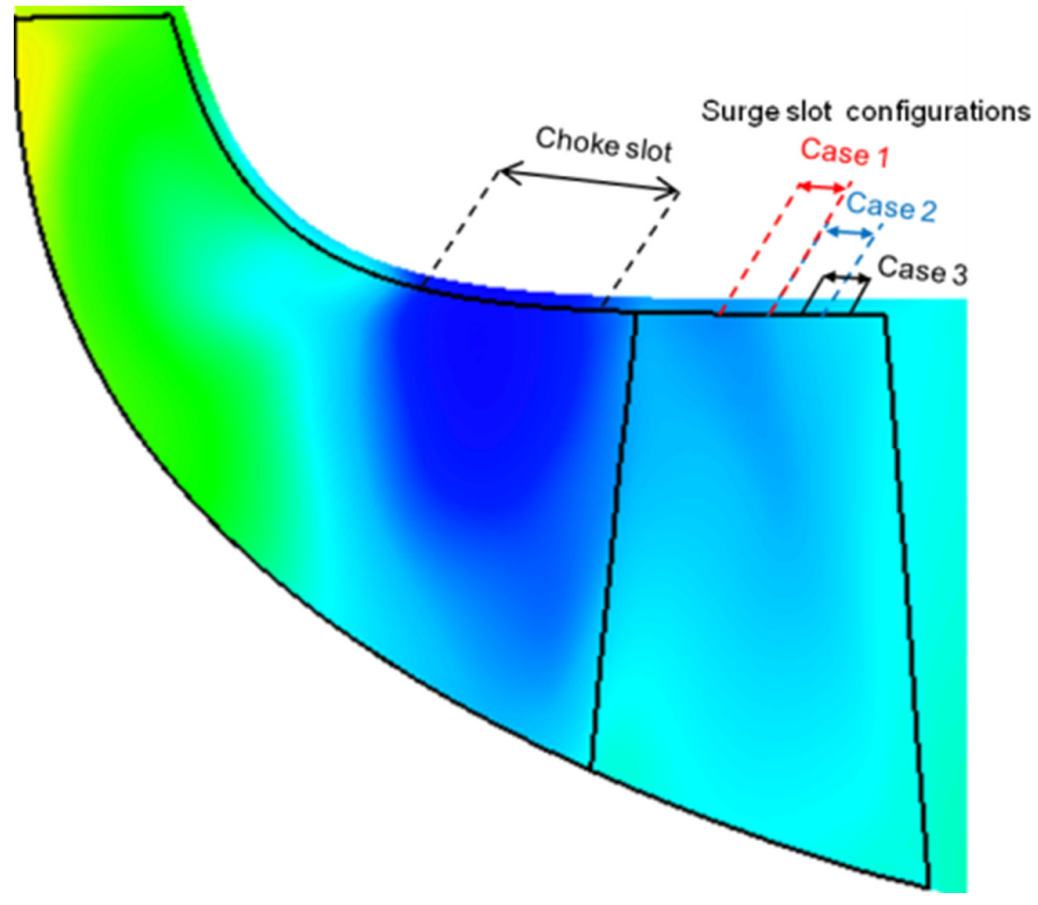

Fig. 4.14 Locations of Dual SRCT Bleed Slot 

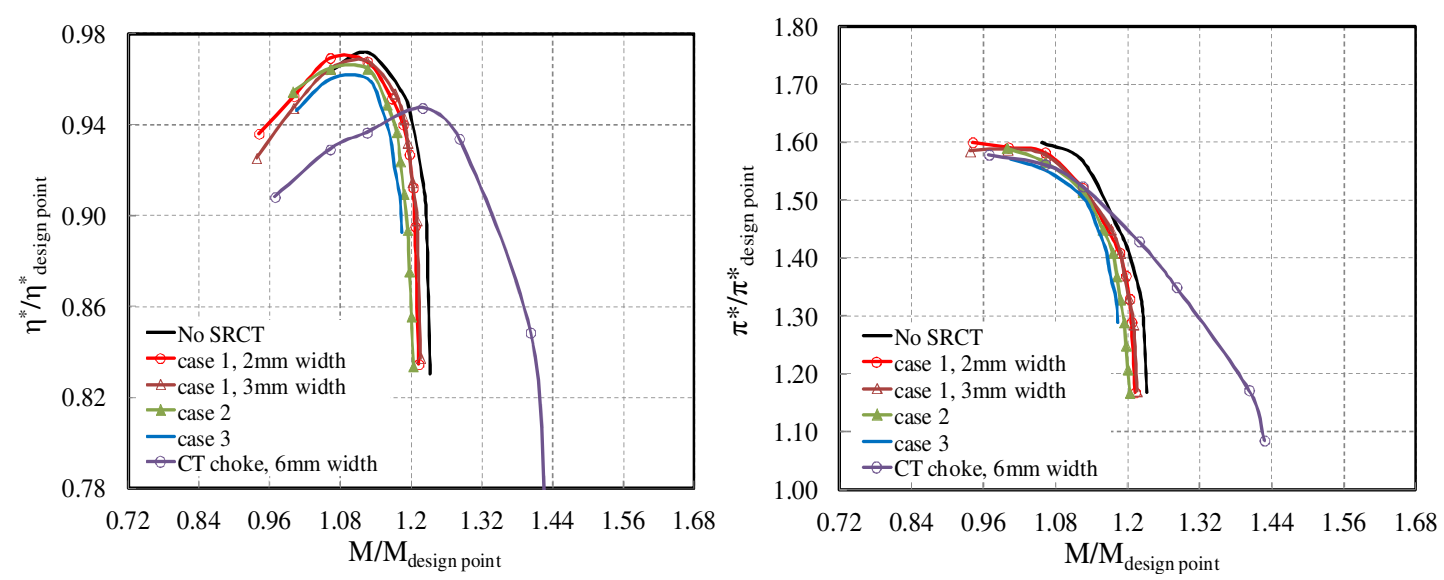

Fig. 4.15 Effect of SRCT's Surge Slot on Compressor Performance

4.6.1 Mechanism of single slot and dual slot casing treatment

None of the existing meanline software that we have used, either Compal from ConceptsNREC or CompAero from TurboAero, are capable of predicting the performance of a casing treatment. Thus the optimal design of active casing treatment is conducted with 3D CFD after the design of impeller is completed, and compressor diffuser and shroud line are fixed.

When Fisher proposed the self-recirculation casing treatment, it was claimed that the recirculation at surge condition may reduce the effective flow area at the impeller inlet, thus reducing incidence and stabilizing the inducer flow and easing the stall. While at choke conditions with the blockage at the blade passage throat, typically at impeller inlet and especially with the inducer at negative incidence, the air builds up a positive pressure drop across the throat and drives the air injection at a point downstream of the inducer throat, thus effectively increasing impeller flow capacity [6]. For decades, it was widely accepted that a self-recirculation casing treatment may help ease the stall at low end and extend the flow capacity at high end, with little efficiency penalty.

This conclusion may not be always true. The effectiveness of a single slot casing treatment taking care of both surge and choke depends on the design of the impeller blade passage. In other words, it depends on where the aerodynamic (not the geometric) blade passage throat is located. If the introduction of splitter blades creates a blade passage throat downstream, especially with the various aerodynamic blockages, the injection of air between the leading edges of main blades and splitter blades at choke conditions may not necessarily help to extend the choke capacity. In this case, the injection slot should be located after the leading edge of splitter blades, where the passage throat is, to gain extra flow capacity. Apparently, a slot after the splitter leading edge is not an optimal location for surge due to efficiency loss. A logical conclusion would be that two slots may be needed to control surge and choke separately. 


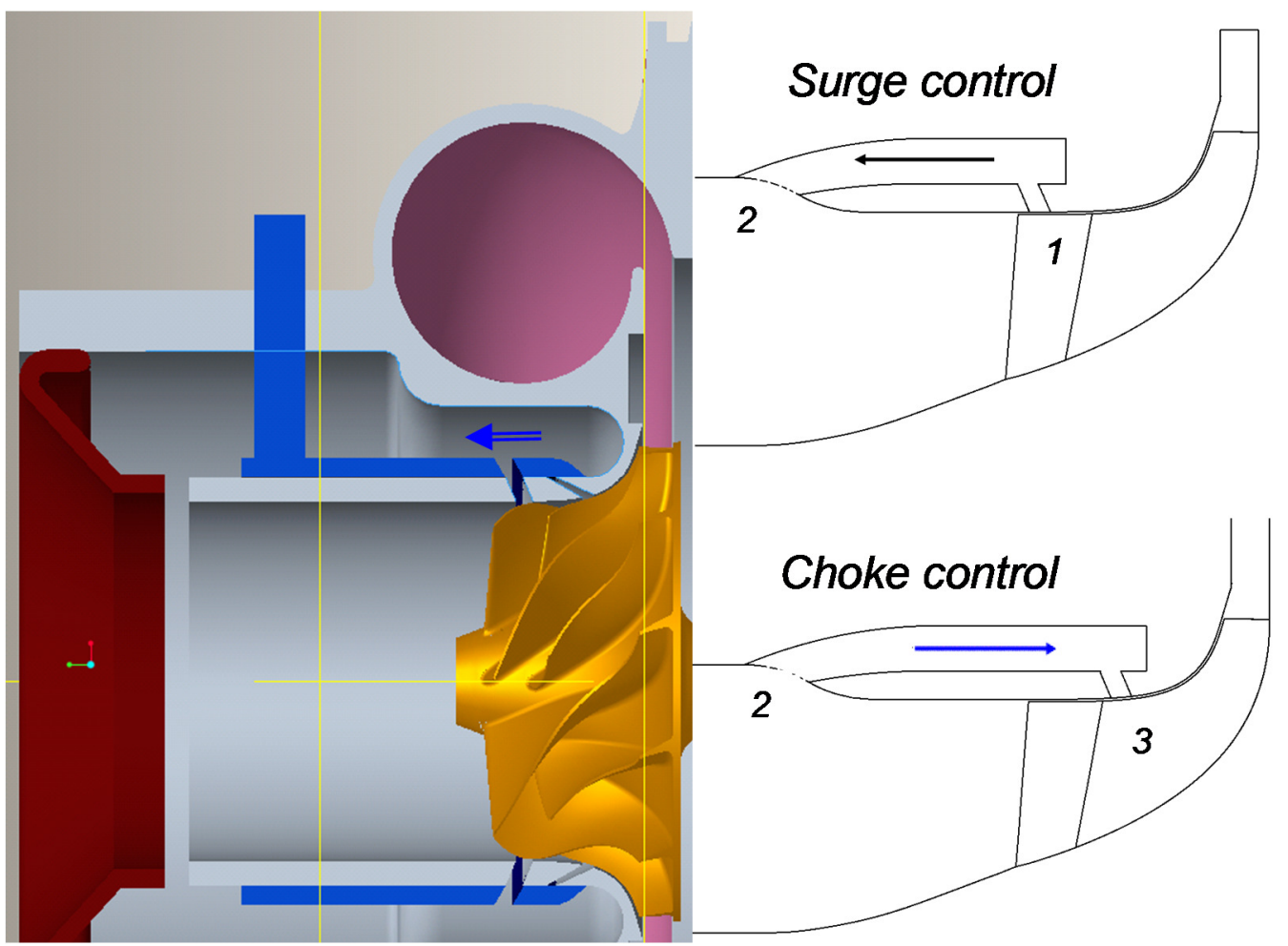

Fig. 4.16. Active Casing Treatment: the bleeding and injection slot before and after splitter blades can be controlled separately to extend surge margin and choke flow capacity.

Conventional compressor impeller design, when single-slot casing treatment is adopted for wide operation range, requires that blade passage throat is located near the leading edge of full blade, i.e., the introduction of splitter blades has to be located downstream. Moving splitter blades far downstream, especially considering the aerodynamic blockage and shock wave induced blockage, will in general compromise the efficiency and surge performance of the impeller at low end. Apparently, to achieve superior performance at the low mass flow area that is heavily weighted in customer driving cycles for light and medium duty vehicle applications, the surge and choke have to be addressed separately, i.e., a switchable dual slot casing treatment or an active casing treatment is needed. Figure 4.16 is one example of such an "active casing treatment." There are two separate slots on the compressor shroud, one slot between full and splitter blades serving as a bleed slot for surge control, and the other slot downstream of the splitter blades serving as an injection slot for choke flow capacity enhancement. These two slots can be covered individually to achieve superior low end efficiency and surge performance, as well as high end choke flow capacity. 


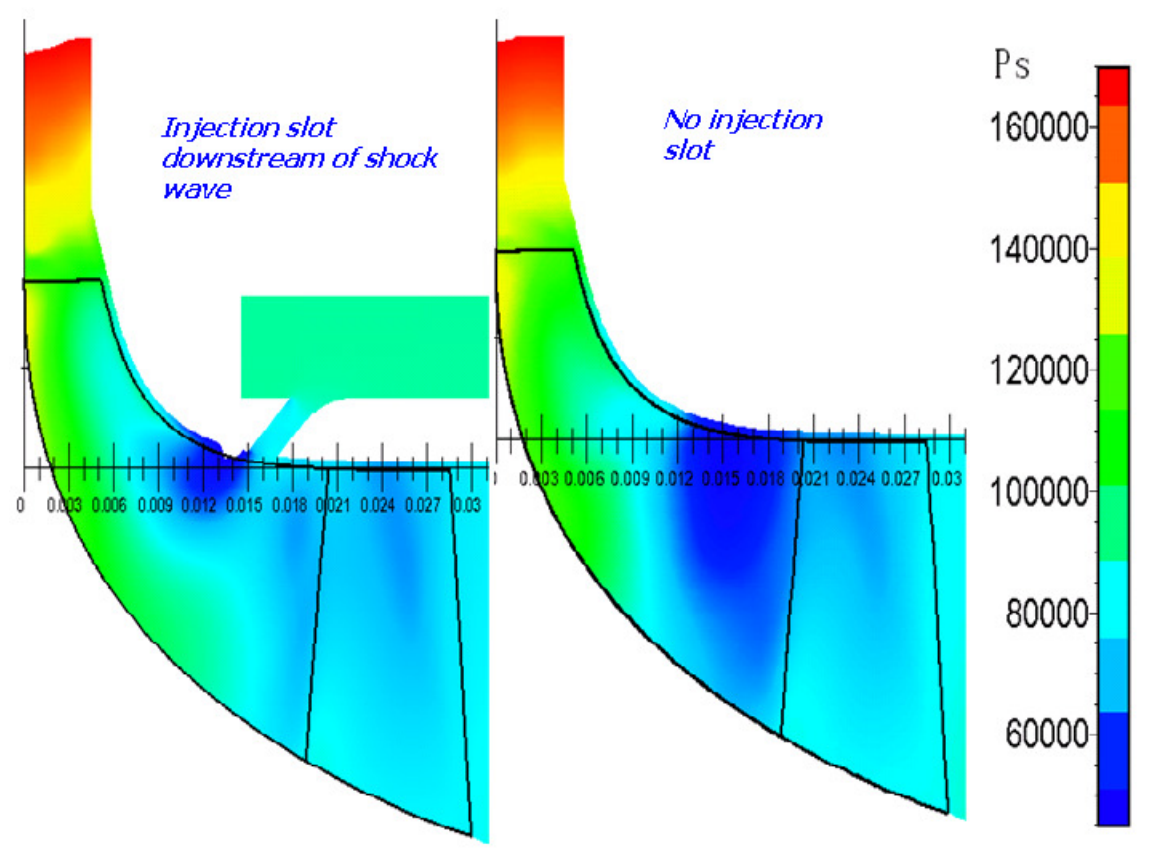

Fig. 4.17. Static pressure distribution near choke condition, which shows the air injection downstream of the shock wave effectively filling up the pressure depression area (azimuthally averaged, from steady state CFD simulation using Numeca)

When the injection slot for choke extension is located downstream of the leading edge of the splitter blades, extra air (5-12\% of total flow, depending on the location and design of splitter blades) can bypass the aerodynamic blade passage throat and the shock wave-induced flow separation. This will directly inject into the pressure depression area (in terms of static pressure) that follows the shock wave, thus effectively extending the choke flow capacity. It is conceivable that decoupling of the surge and choke control can not only extend choke flow capacity but can also enable further improvement of surge and low end efficiency by the optimal design of impeller leading edges of full blades, surge slot, as well as splitter blades and also the location of splitter blades.

Comprehensive numerical optimization, including injection slot location, width, orientation, etc., has been conducted (as described in detail in section 4.4 and 4.5) before a final version of the dual slot, or active, casing treatment was designed, fabricated and flow bench tested. Figure 4.16 shows the CFD simulation of the flow field of static pressure distribution near choke condition of a compressor without casing treatment and with an injection slot that was optimized for choke flow capacity extension. Without casing treatment, the static pressure drops down to $40 \mathrm{kPa}$ below ambient following the shock wave near the leading edge of the splitter blades. The injection of extra air downstream of the shock wave near choke conditions effectively fills up the pressure depression area thus extends choke capacity.

\subsubsection{Conventional Single-Port Casing Treatments:}

As part of an ongoing effort to increase the usable compressor operating range a handful of compressor design variants have been explored in depth. Extensive CFD evaluations have been conducted on many of the impeller designs. (Introduction of the CFD methodology used in this study has been the subjects of prior publications [43, 44]). 
Modern turbocharger compressor impellers typically operate in the so called super critical regime with supersonic inlet relative Mach number along the shroud (Figure 4.18.) at the high-flow rated point.

As described by Fisher [6], the classic SRCT can augment the performance map both at high and low flow rates due to the change in local static pressure along the shroud wall at the bleed slot location. The conventional approach to self-recirculating casing treatment (SRCT) has only one slot that serves as a bleeding slot (out of the main stream into compressor cover) during low-flow operation and injection slot (into the main stream from compressor cover) during high-flow operation. Typically, conventional recirculation casing treatment design has been focused on balancing the trade-off between low end surge improvement and maximum flow capacity, optimizing the location, orientation and size of the single slot.
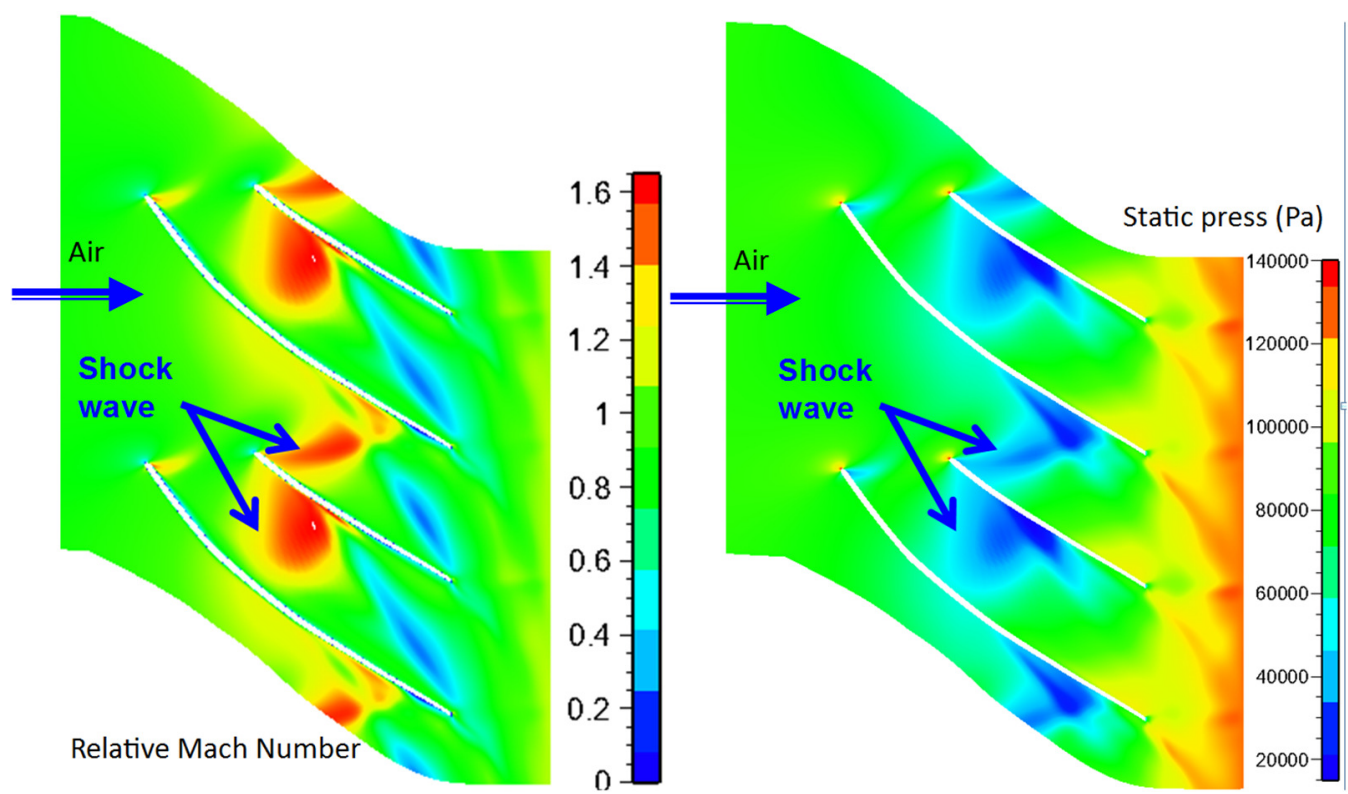

Fig. 4.18 Relative Mach number and static pressure distribution at 95\% span near maximum flow capacity condition indicating high Mach number region and low static pressure region (based on steady state CFD simulation using Numeca [44])

Figure 4.19 illustrates the CFD predicted flow field of a transonic compressor impeller near choke condition. At $85 \%$ of spanwise along the meridional patch, increase of relative Mach number is accompanied by a decrease in static pressure (Fig. 4.19c). Based on this CFD simulation, a new compressor design concept is introduced that is substantially different from conventional practices for a transonic impeller design that have been used for a few decades, i.e. to design the impeller based on the geometric flow passage area, taking into account of aerodynamic blockage and most importantly, to utilize the aerodynamic blockage due to introduction of splitter blades or, in other words, the pressure depression right after the shock wave, to inject additional flow, bypassing the blade passage throat, thus extending the compressor operation range without compromising efficiency. Compared with conventional injection slot for casing treatment, the post-shock wave injection can suck in much more air, thus more effectively extending the choke flow capacity. 


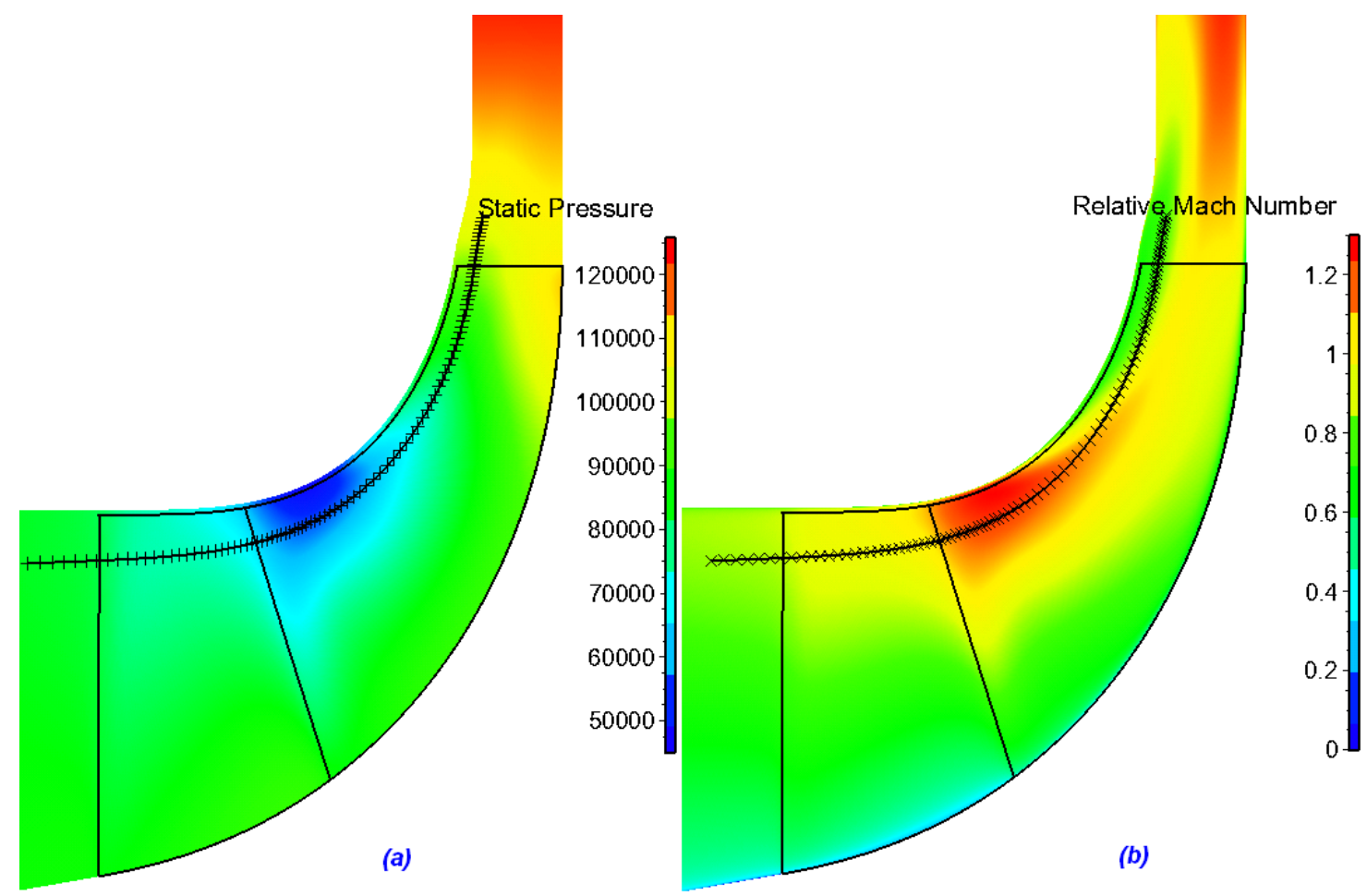

Fig. 4.19a and 4.19b. CFD (azimuthally averaged) prediction of static pressure (a) and relative Mach number (b) distribution inside a transonic compressor impeller near choke condition $[43,44]$

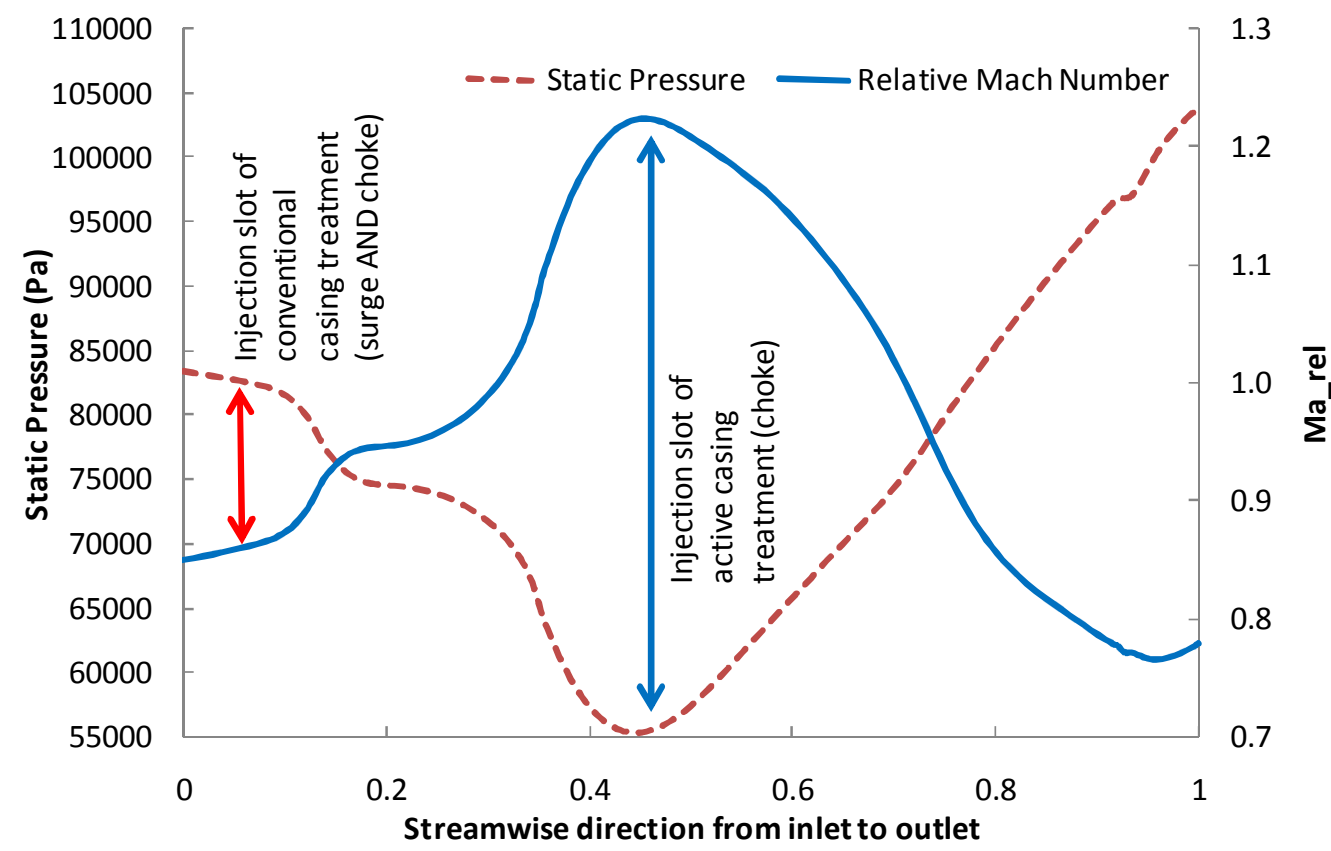

Fig. 4.19c. CFD prediction: variation of static pressure and Mach number at $85 \%$ of spanwise, along streamwise direction of a transonic compressor impeller near choke condition $[43,44]$

The steady state numerical prediction of the choke extension of this active casing treatment is fairly well validated by the flow bench test, despite the challenges of transonic 
CFD simulation, especially the interfacing between stator and rotor near the injection slot [37].

The CFD simulation shows that active casing treatment can enable optimal design of surge slot to have improved efficiency and surge margin at low end while extending choke flow capacity at high end when the choke is opened. According to the CFD analyses (Fig. 4.20), the choke flow capacity extension depends on the pressure ratio required at rated speed. For most of the turbocharged chassis cert diesel and gasoline engine, the pressure ratio at rated would be between 2.5 and 3.0 thus up to $12 \%$ choke flow capacity increase is possible via the active casing treatment; while for some diesel applications that either requires EGR at full load or has high power density or poor volumetric efficiency at rated speed thus requires high boost (e.g. above 3.5) at rated condition, the benefit of an active casing treatment will be limited. 

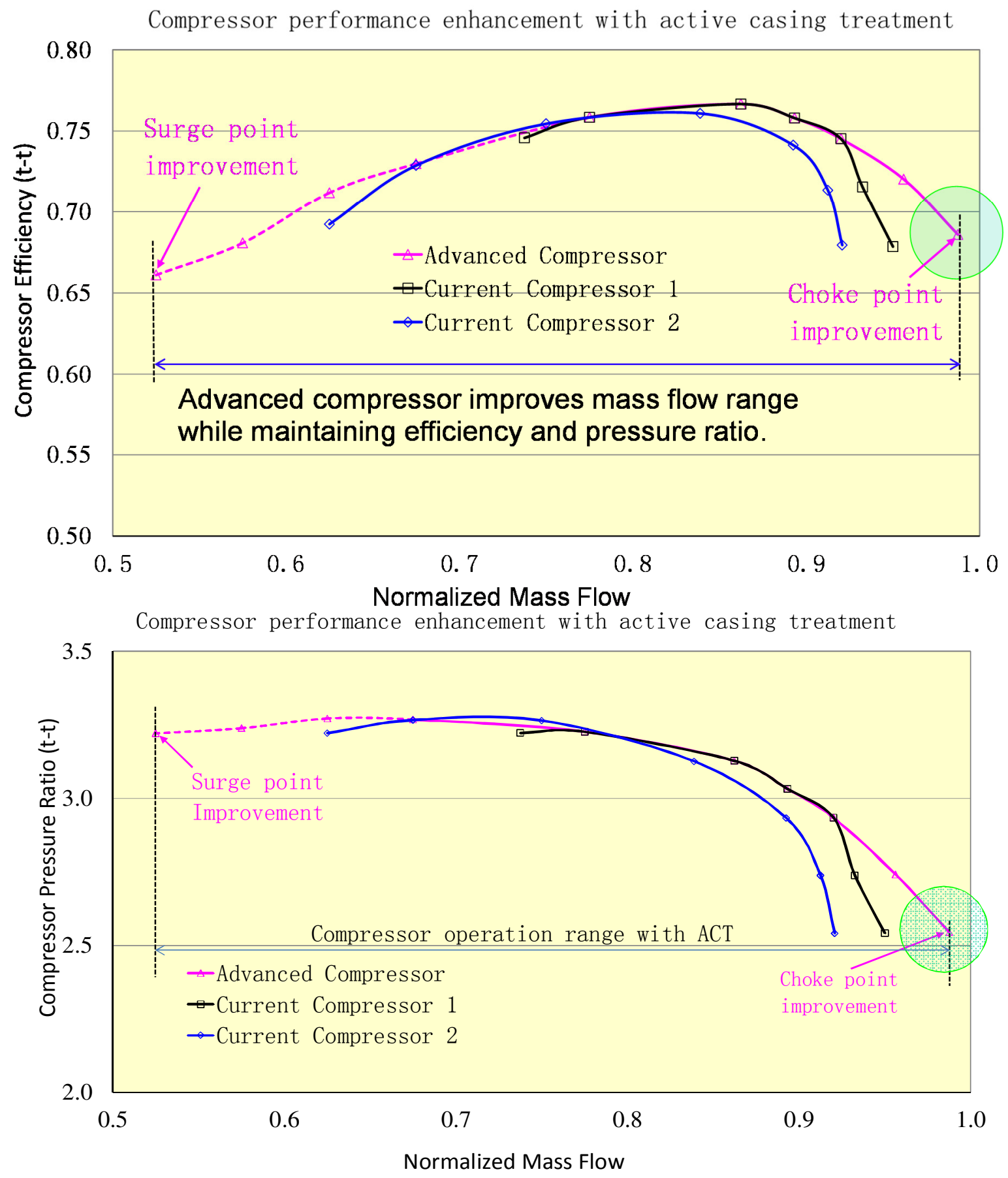

Fig.4.20. Comparison of CFD performance prediction of advanced MD compressor and two production compressors operating at medium turbo speed ("current compressor 2" is the donor compressor) 


\section{7}

Stress and structure analyses of the advanced MD Compressor:

Typical compressor development process will include stress and structure analyses to ensure that there is no high cycle and low cycle fatigue concerns. The Ansys Mechanical software was used to predict the stress distribution at burst speed. Both the peak blade stress on the splitter blade and the peak bore stress were predicted below material yield strength with sufficient engineering safety margin (Fig. 4.21). The peak blade stress is located in the center of impeller, close to leading edge that is typically exposed to lower air temperature. The arbitrary surface of the impeller helps to gain more reasonable stress distribution. Exposing to lower temperature, the bore area typically allows higher stress level. In the current MD impeller design, the peak bore stress is $10 \%$ higher than peak blade stress, which is typically acceptable. Should the bore stress be a concern when matching with a different center housing that requires a different compressor back face profile, a boreless or an optimal back face profile design can be pursued to reduced bore stress. Wrought aluminum 2618-T61 was assumed for the milled compressor impeller.

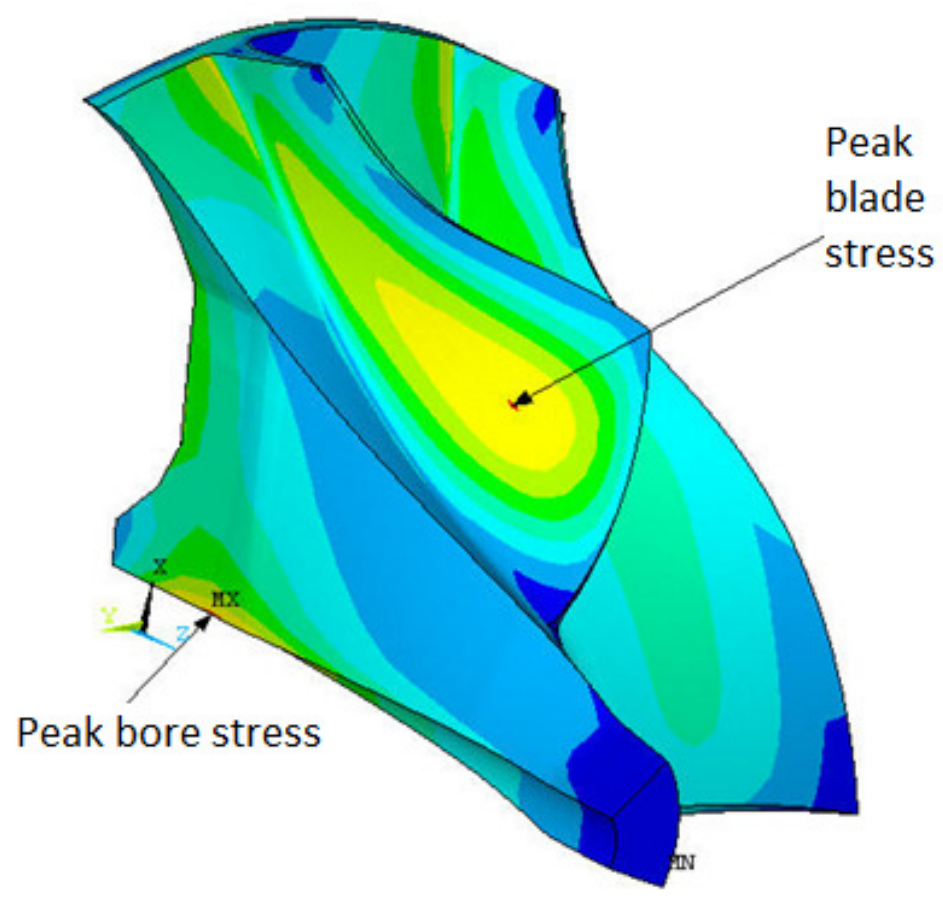

Fig.4.21. Stress distribution of advanced MD compressor impeller 


\section{Campbell Diagram}

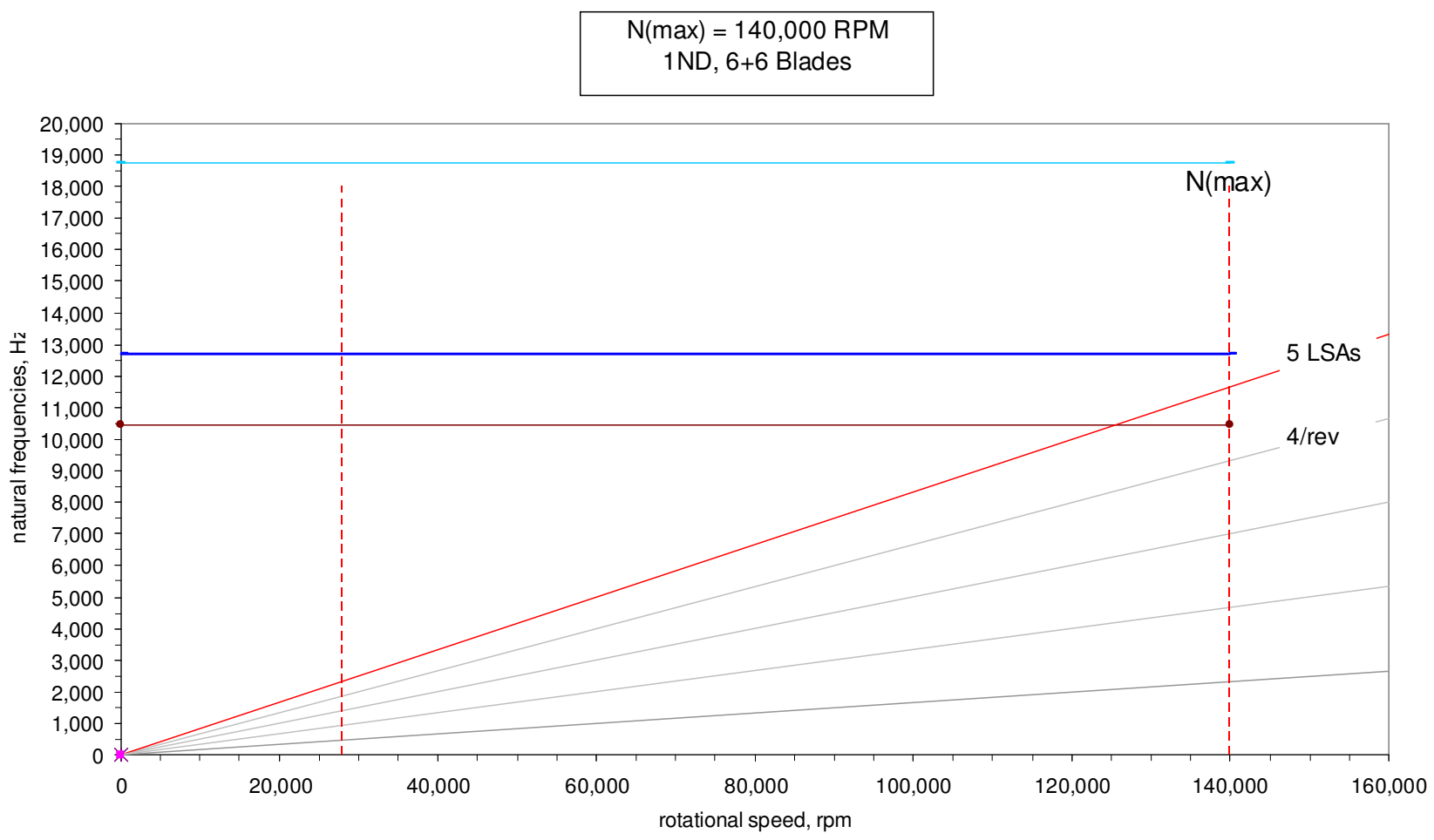

Fig.4.22. Structure analysis of MD compressor impeller

The modal analysis of the MD compressor impeller (Fig. 4.22) shows that the first order of natural frequency is about 4.68 times of max operation speed of the impeller, which is sufficient for a milled compressor impeller. For the modal analysis, the axial displacement was set to zero at the bore center of back face of the compressor wheel.

The stress and modal analyses illustrated in Fig. 4.21 and 4.22 represent the final design of the MD compressor impeller, after 18 design iterations between 1D, 3D design optimization, CFD performance validation and CAE stress and structure analyses, following the design optimization process described in Fig. 2.1

\subsection{Three dimensional design and CFD/CAE analyses of MD mixed flow turbine}

On the turbine side, the mixed flow turbine has been introduced 50 years ago at then NACA [46], initially as an alternative rotor design for gas turbines. It was well received that the mixed turbine has performance advantages operating at high pressure ratio condition, e.g. high pulsation flow such as in automotive applications, i.e. the mixed flow turbine can extract more exhaust energy. N. Walson and M. S. Janota has summarize this benefit of mixed flow turbine in their book "Turbocharging the internal combustion engine [32]. Since then the mixed flow turbine has drawn great attention for high efficiency over wide speed ratio U/C.

The turbine speed ratio is defined as 


$$
\frac{U}{C}=\frac{U}{\sqrt{2 C_{p} T_{0}\left[1-\left(\pi_{T}\right)^{-0.285}\right]}}
$$

As illustrated by Fig. 1, the recent emission compliant diesel engine spends more and more time in the low $\mathrm{U} / \mathrm{C}$ area, compared to a turbocharged diesel before EGR was used. That means that the turbine efficiency has to be improved at low $\mathrm{U} / \mathrm{C}$ even though the peak efficiency may not be improved, which is exactly the mixed flow turbine can do [34].

Initial intention of mixed flow turbine designed in the 1970s by Wallace and Pasha [50] was to increase the swallowing capacity. The initial design had a modified radial turbine with mean cone angle of $45 \mathrm{deg}$. Since current MD mixed flow turbine has to fit into the variable geometry nozzle mechanism of the donor turbocharger that was designed with a radial flow turbine. Thus the mean cone angle is somewhat compromised and less aggressive than what we'd like to have without the constraint of the variable geometry nozzle mechanism. Figure 4.23 is a CAD model of the advanced MD mixed flow turbine with a radial flow VGT.

By the 1990s, Chen and Baines managed to reduce the velocity components at the inlet and the exit of the mixed flow turbine blade [47]. By nature, mixed flow turbine may have higher inertia, due to larger hub diameter, than radial flow turbine. The larger swallowing capacity associated with missed flow turbine allows slightly smaller turbine diameter thus off set the inertia increase. Similar to 3D compressor design, numerical analysis and flow field visualization (Fig. 4.24) using 3D CFD tools were extensive used for

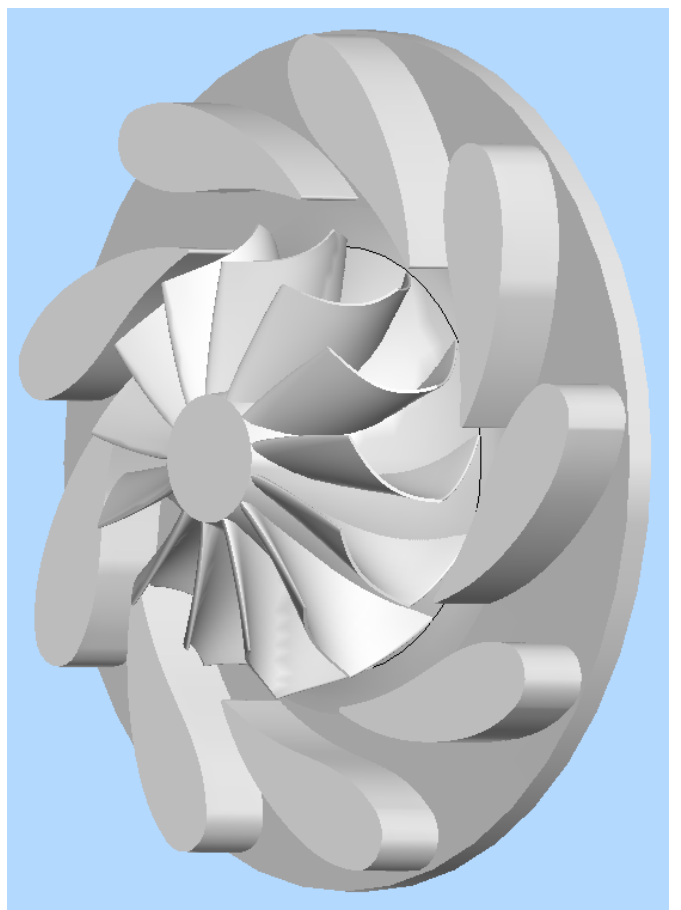
design optimization and performance validation. However, one has to understand the challenge in comparing the 3D CFD predicted turbine efficiency, typically without bearing loss, with either the current turbine performance map or the design targets listed in Table 1.1, which typically carry bearing loss.

Fig.4.23. Design of advanced mixed flow turbine that was matched with radial flow variable geometry nozzle mechanism of the donor turbocharger 


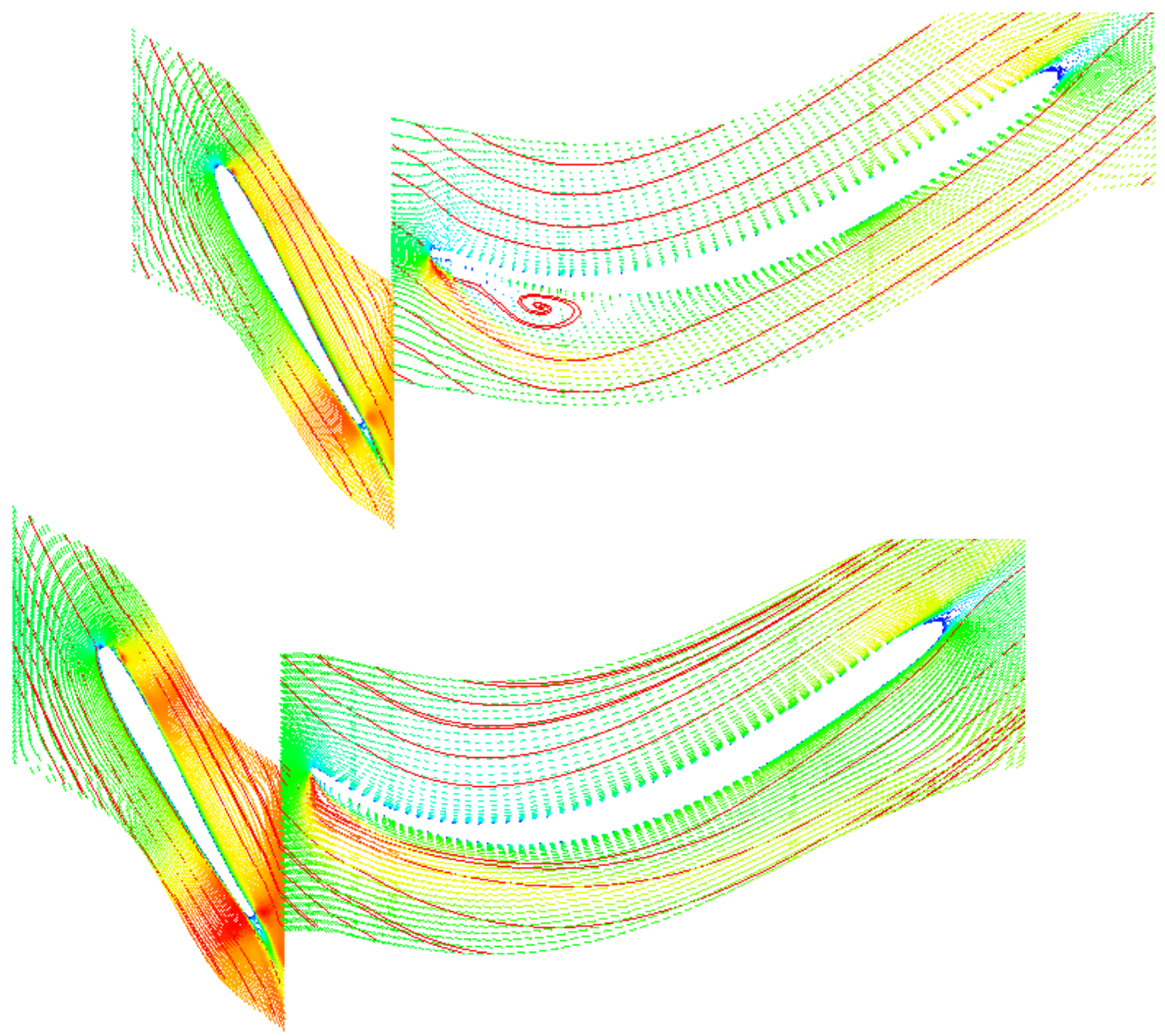

Fig.4.24. Steady state CFD visualization of entropy distributions of R3 (top) and R5 (bottom) designs of mixed flow turbine (the flow separation in R2 inducer area indicated flow loss)

Current design of the advanced MD mixed flow turbine utilizes the VGT mechanism and volute of donor turbo, thus the maximum flow capacity at maximum vane open position remains unchanged. The design priority is to improve the turbine efficiency at full flow rate so the turbine inlet temperature limited engine power can be increased. It is conceivable that mixed flow turbine will have a shroud line drastically different to that of a radial flow turbine of the donor turbocharger. Therefore, a specially designed shroud insert is used inside the turbine housing. 


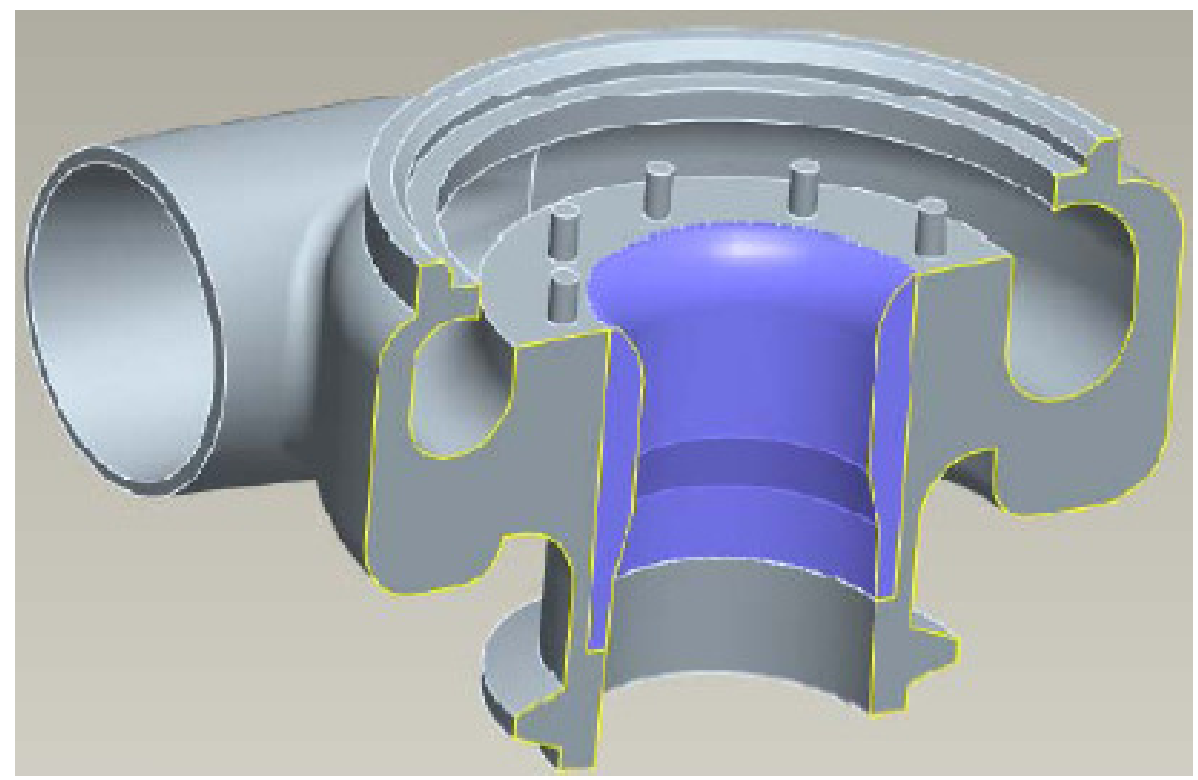

Fig.4.25. Design of shroud insert of advanced MD mixed flow turbine to fit into donor turbine housing

The other challenge in turbine performance correlation with turbine performance map is that typical turbine performance map out of steady state flow bench test is constrained to a small operation range, i.e. the pulsed flow from exhaust gas of internal combustion engine would push the operation point into the area that cannot be characterized by typical steady state flow bench tests. Therefore CFD, especially the transient CFD simulation is very useful in comparison of different turbine performances. Figure 4.26 is the steady state and transient CFD performance predictions of advanced MD mixed flow turbine, compared with conventional radial flow turbine at $80 \%$ VGT open position. Both the steady state and transient simulation over one engine firing cycle indicate that the turbine efficiency improvements are mainly in low $\mathrm{U} / \mathrm{C}$ areas. The mixed flow turbine also shows $4 \%$ extension in swallowing capacity, even though this particular design of the mixed flow turbine has the same inertia as radial flow turbine. 
Turbine efficiencies, radial flow vs. mixed flow under stead state and transient conditions

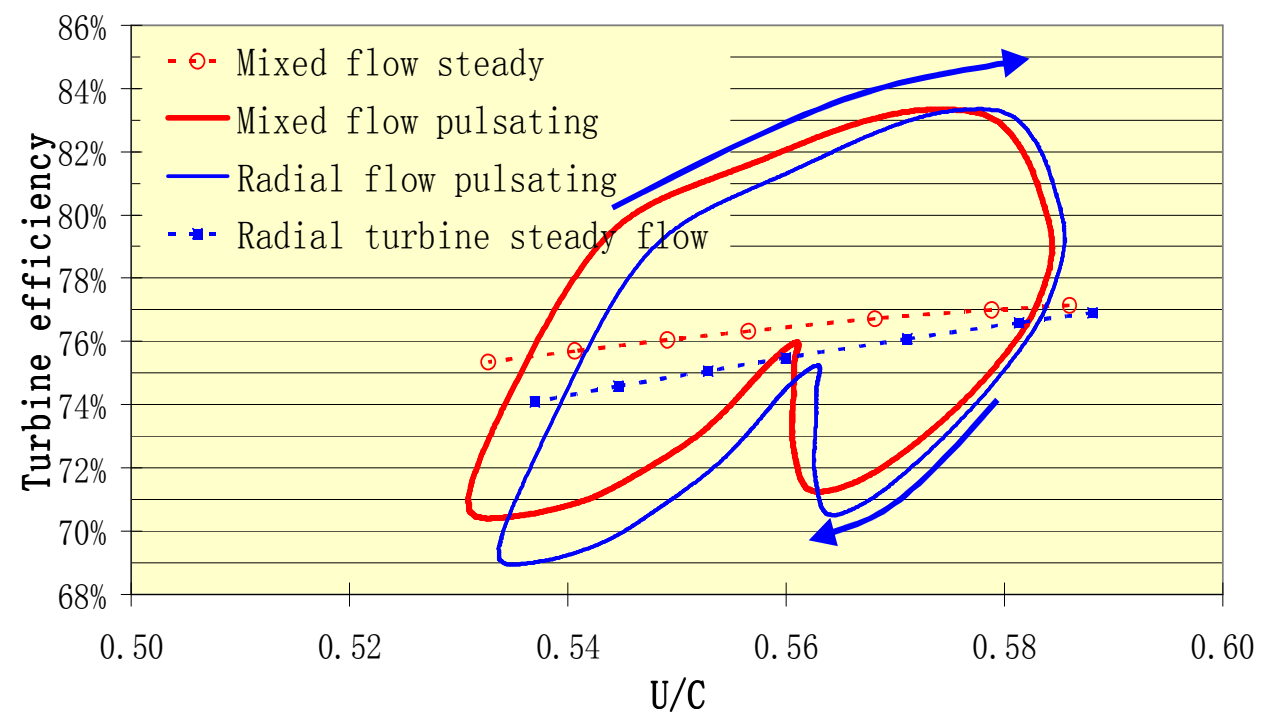

Mass flow of mixed flow turbine vs. radial flow turbine

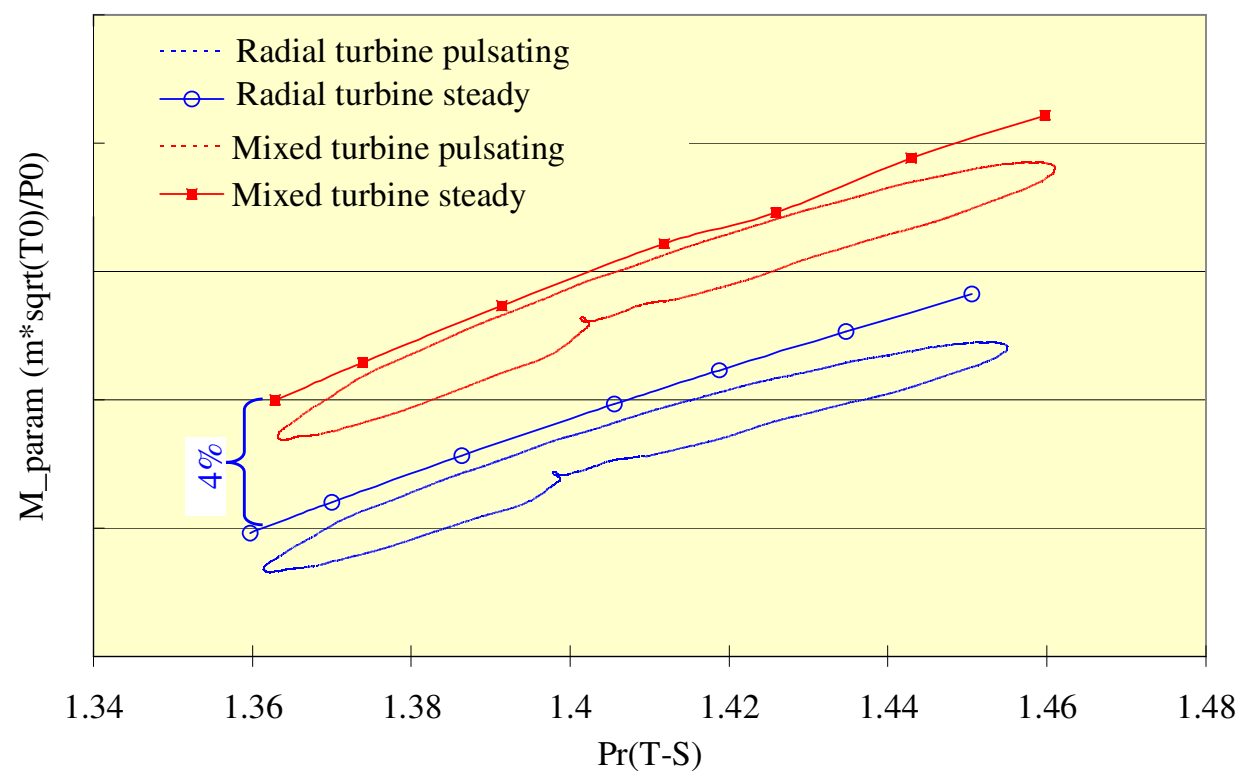

Fig.4.26 Steady state and transient CFD performance predictions for advanced MD mixed flow vs. radial flow turbine 
Similar to compressor development, the 3D design of advanced MD mixed flow turbine also need to go through a series of 3D CAE stress and structure analyses after the 3D CFD performance validation that supports meanline performance predictions in terms of efficiency and flow capacity.

Figure 4.27 illustrates the Ansys predicted von Mises stress distribution of the advanced MD mixed turbine wheel under high temperature and at rated speed. In the stress analysis, Inconel 713LC material was assumed for the turbine wheel. Also uniform $770 \mathrm{deg} C$ skin temperature on the blade surface was assumed. The temperature at the center of rotating shaft at oil seal area was set to $100 \mathrm{deg} C$. The initial design (design 1), matching to the donor turbine back face profile, shows very high back face stress. Typical approach to address the high back face stress is to use scallop cut which typically involves turbine efficiency degradation (2-3\% possible, according our CFD analyses). After 10 design iterations in the back face design, the optimal design 2 shows $30 \%+$ reduction in von Mises stress (Fig. 28).

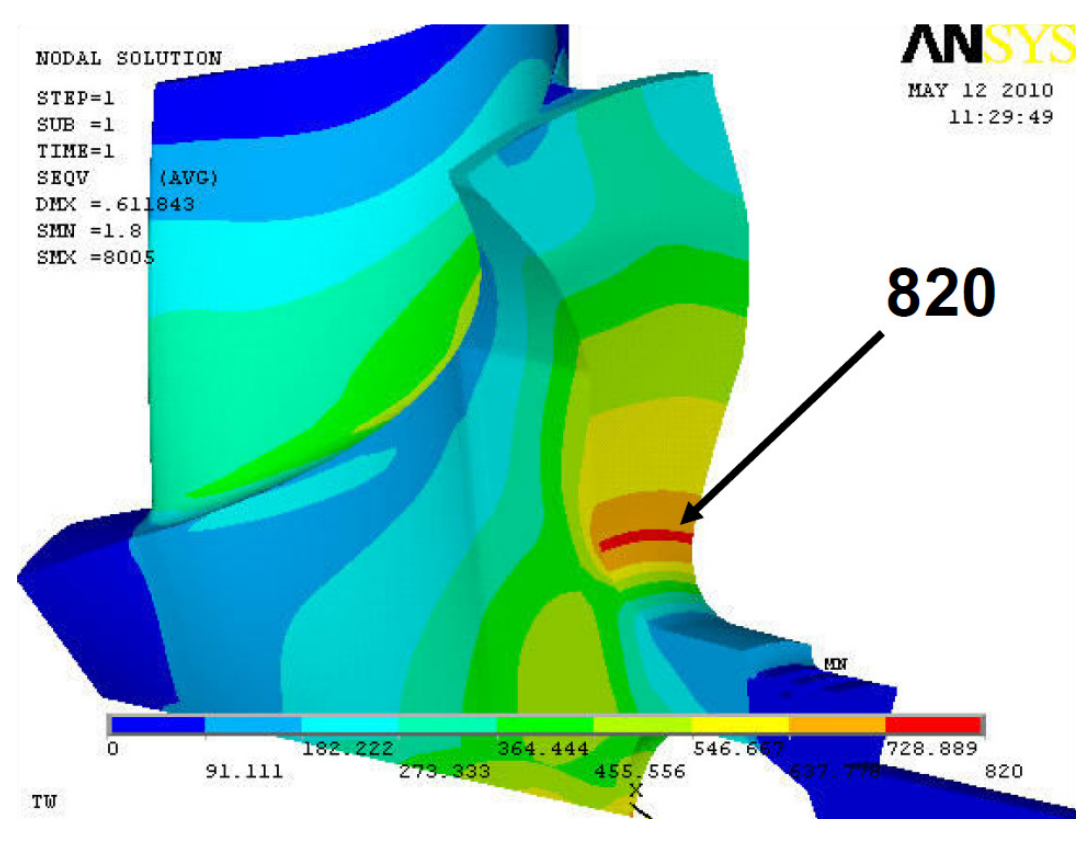

Fig.4.27. von Mises stress of mixed flow turbine at 135krpm of design 1, showing the high stress in the back face due to mating with the donor center housing design

For the modal analysis, the axial displacement was set to zero at the bore center of rotational shaft at the oil seal area. The modal analysis, as shown in the Campbell Diagram (Fig. 4.29), indicated that the $1^{\text {st }}$ order and $2^{\text {nd }}$ order natural frequencies are $10,442 \mathrm{~Hz}$ and $23,073 \mathrm{~Hz}$, respectively. Since the donor VGT has 9 nozzle vanes, the excitation of $9 /$ revolution would create $1^{\text {st }}$ order and $2^{\text {nd }}$ order of resonance speeds of $73,567 \mathrm{rpm}$ and $154,280 \mathrm{rpm}$, respectively. Apparently the $1^{\text {st }}$ order of resonance speed is right in the center of turbine operation range, which is typical for automotive turbochargers, while the $2^{\text {nd }}$ order of resonance speed is above the rated speed. The rated operation frequency is 4.6 times of the $1^{\text {st }}$ order natural frequency, which is at the borderline for a cast Inconel turbine wheel. However, a Ford proprietary technology [48] that could avoid 
extended operation near the resonance frequencies would make it not necessary to pursue high ratio of operation frequency to natural frequency.

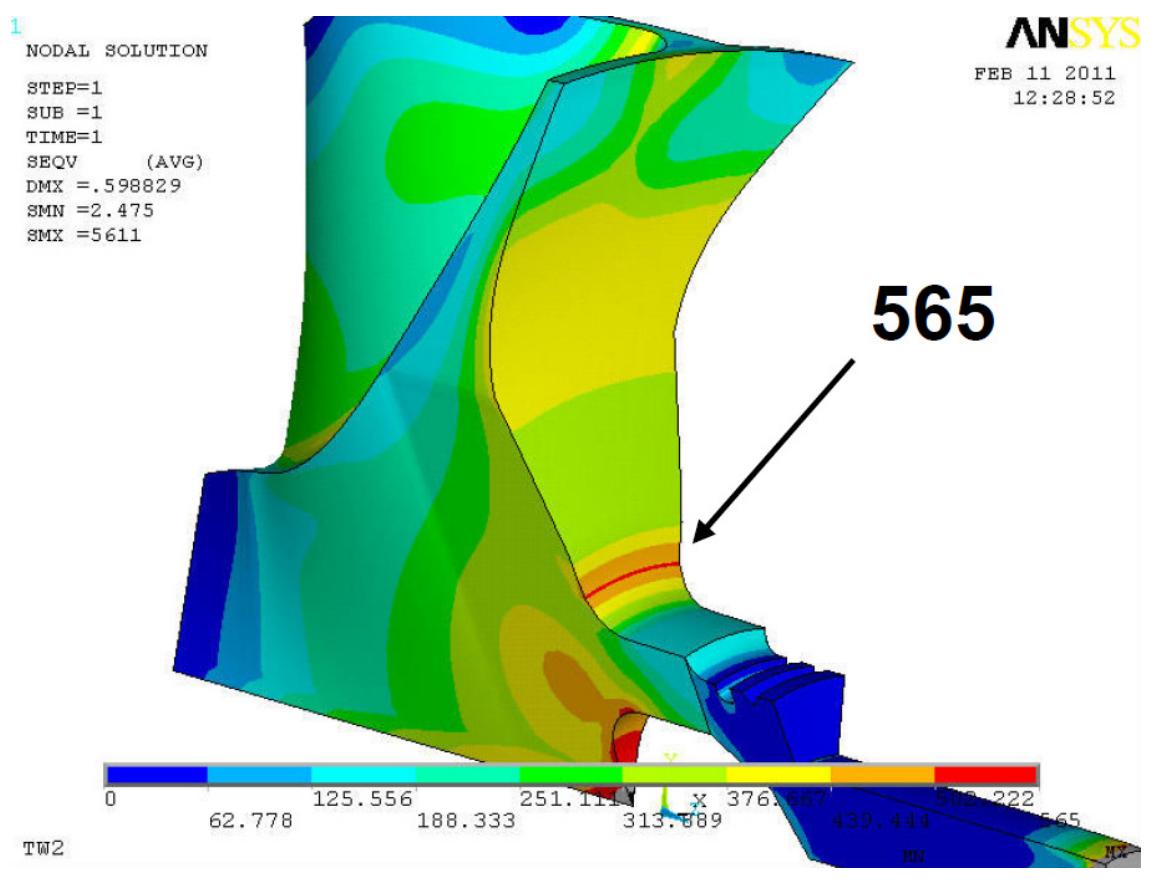

Fig.4.28. von Mises stress distribution of mixed flow turbine at 135krpm of design 2, showing the significant reduction in back face stress after back face profile optimization that deviates from the donor center housing design, compared to Fig. 4.27

\subsection{Rotor dynamics analysis of advanced MD turbocharger assembly}

Both the advanced compressor and mixed flow turbine have slightly longer axial length, the center of gravity of these two rotational group may have certain level of impact on the rotor dynamics, which has to be evaluated via 1D rotor dynamics. Fig. 4.30 illustrates a simplified model of the donor turbo with newly designed advanced MD compressor impeller and mixed flow turbine wheels. The station \#3 and station \#24 represent the compressor inlet plane and turbine exit plane. The simulation shows that at 
200 deg $C$ oil temperature, the radial displacements are under 0.0018 inch or $0.046 \mathrm{~mm}$ at compressor inlet plane and under 0.00096 inch or $0.024 \mathrm{~mm}$ at the turbine exit plane, which are well below the typical tip clearance of either the compressor wheel or the turbine wheel. The peak of vibration occurs near 28,000 rpm (Fig. 4.31). The compressor and turbine response in the frequency domain (Fig. 4.32) shows that the spectrum of peak amplitude of vibration occurs at $15,400 \mathrm{cpm}$ or $257 \mathrm{~Hz}$.

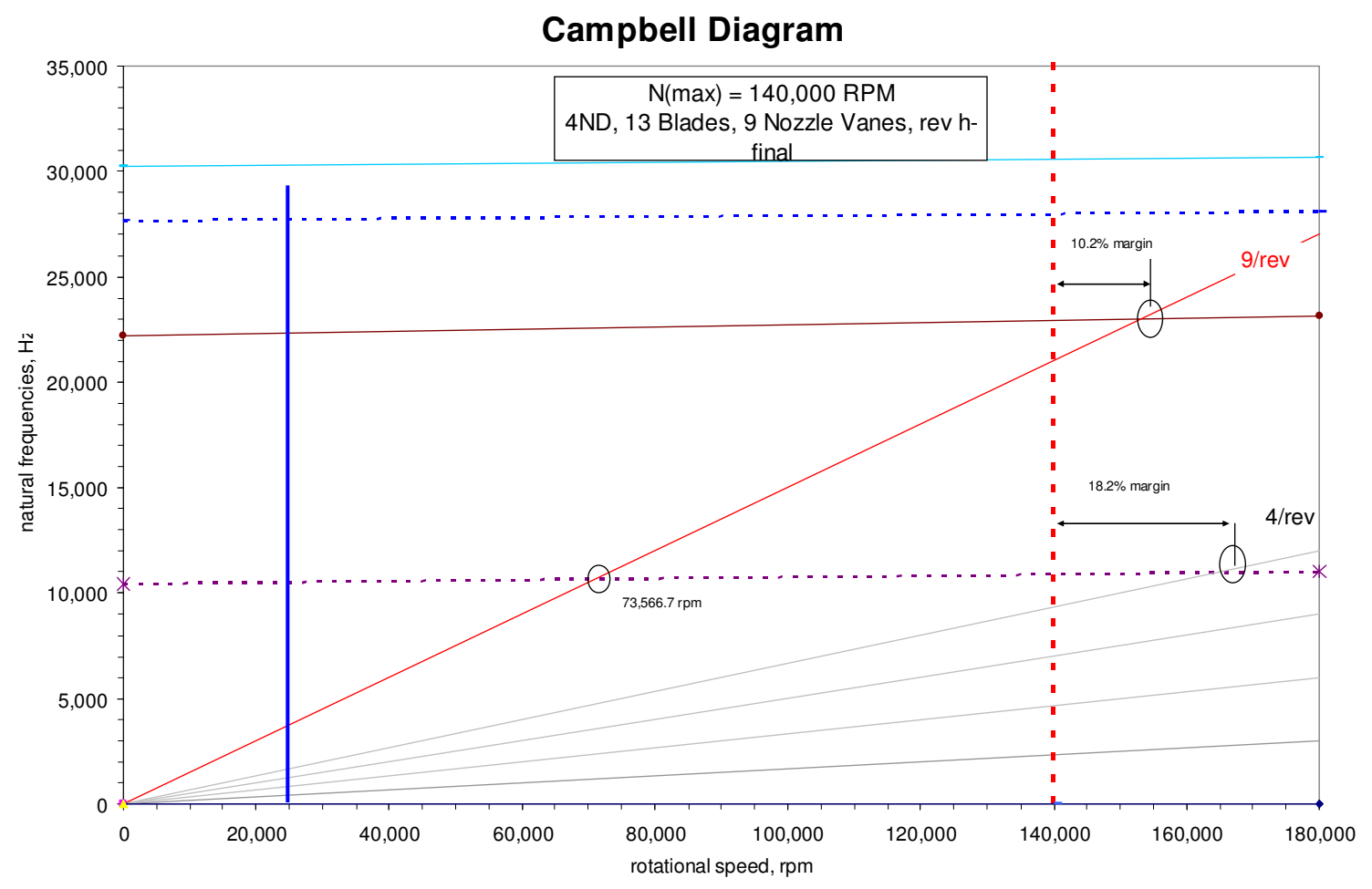

Fig.4.29. Campbell diagram of advanced MD turbine wheel (the VGT has 9 nozzle vanes thus 9 excitations on the blades) 


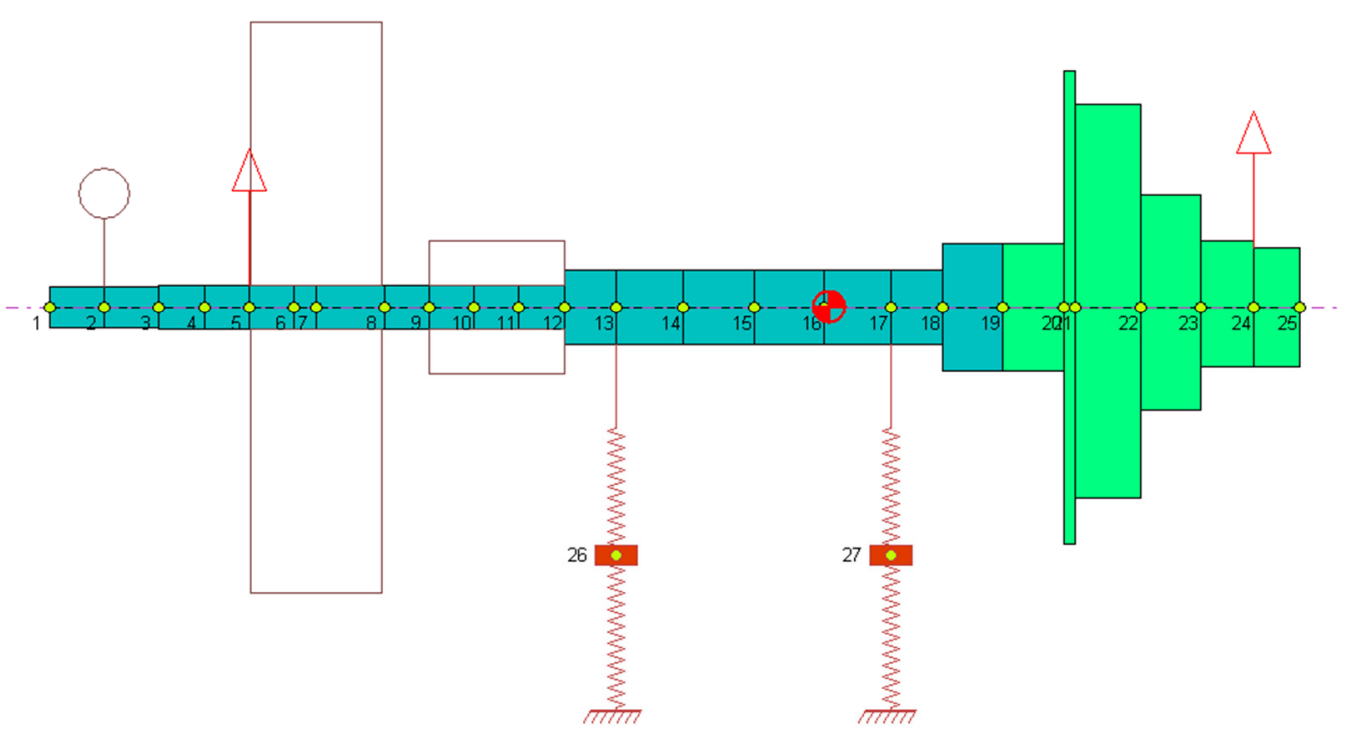

Fig.4.30. One dimensional model for rotor dynamics analysis of the advanced MD turbocharger

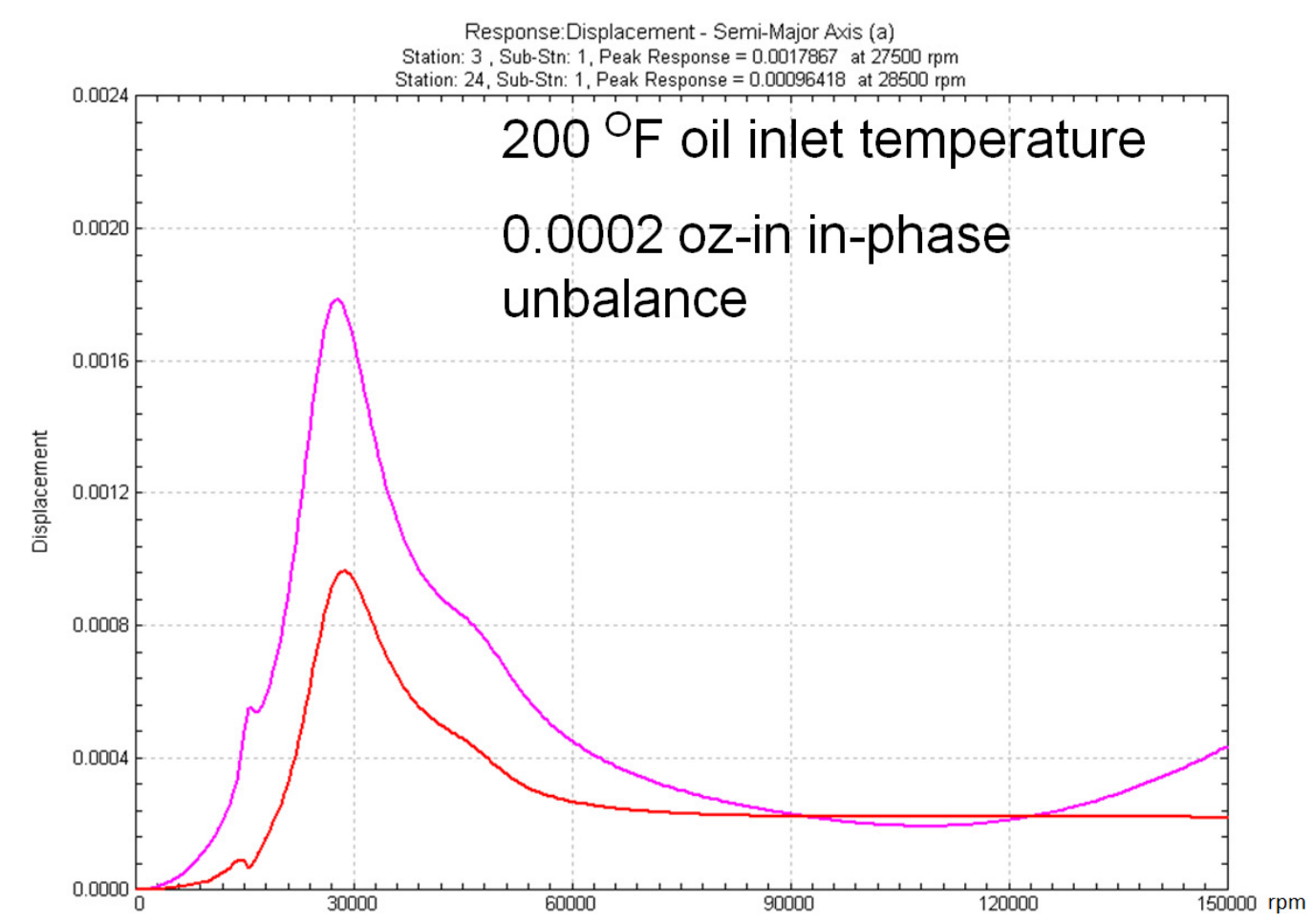

Fig.4.31. Response displacement of the turbocharger rotor at different turbo speeds 

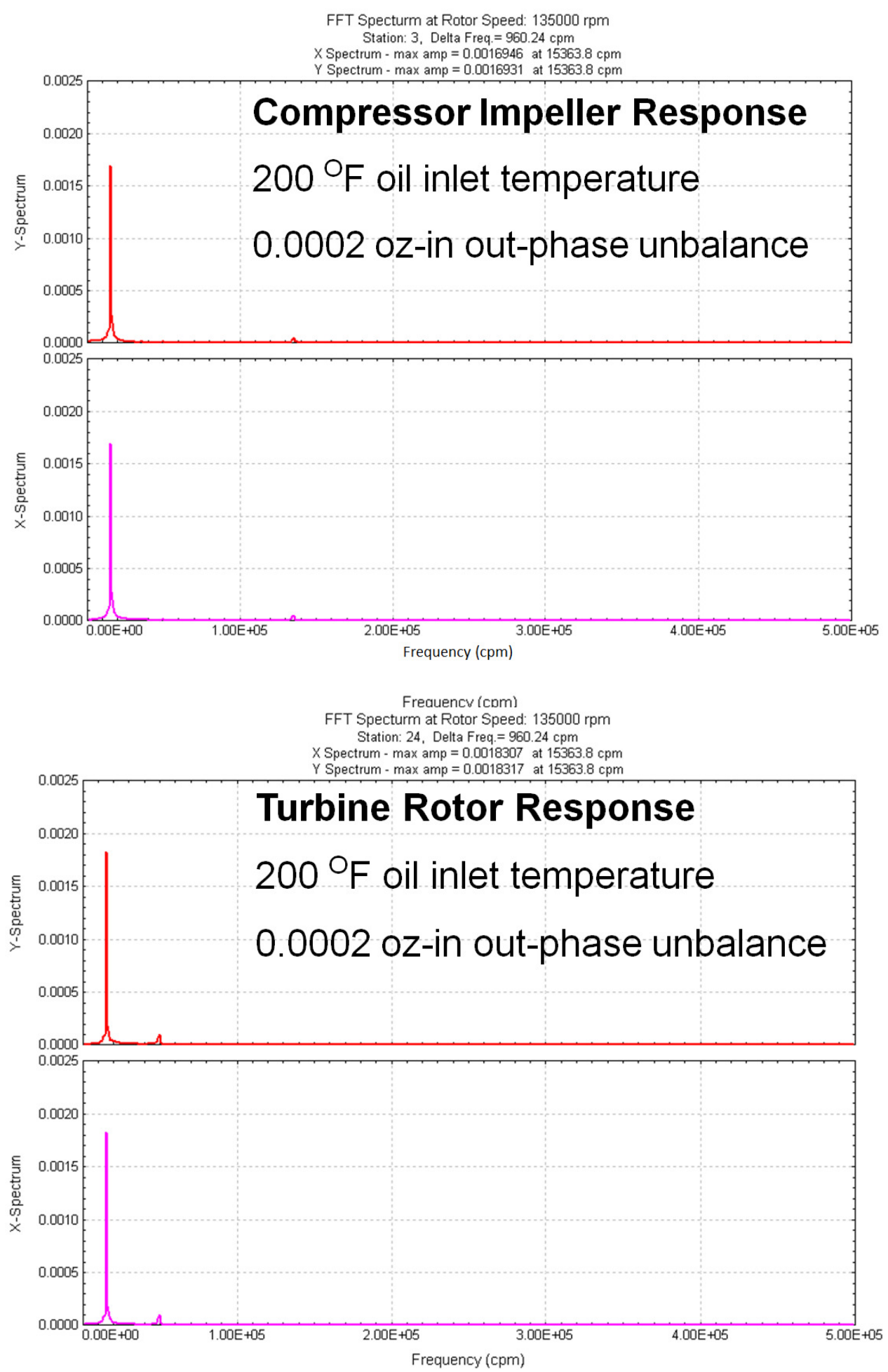

Fig.4.32. Compressor and turbine spectrum of peak amplitude of vibration 


\section{Actuation system design}

The invention of Active Casting Treatment on compressor side [35] makes the control and actuation system development much easier than what was planned in the project, since, instead of variable diffuser vanes or variable inlet guide vanes on the compressor to extend compressor flow capacity as originally planned in the DOE project, only an on-off valve is needed to switch between surge slot and choke slot casing treatment. Based on the concept that is highlited in Fig. 4.16, an electronically controlled and pnuematically actuated active casing treatment mechnism (as shown in Figure 5.1) is designed and packaged into production engine. Since the choke slot needs to open for the last $\sim 15 \%$ of the flow range (the remaining of the $85 \%$ area closed), the current wastegate control signal from the Scorpion engine can be utilized to open the choke slot.

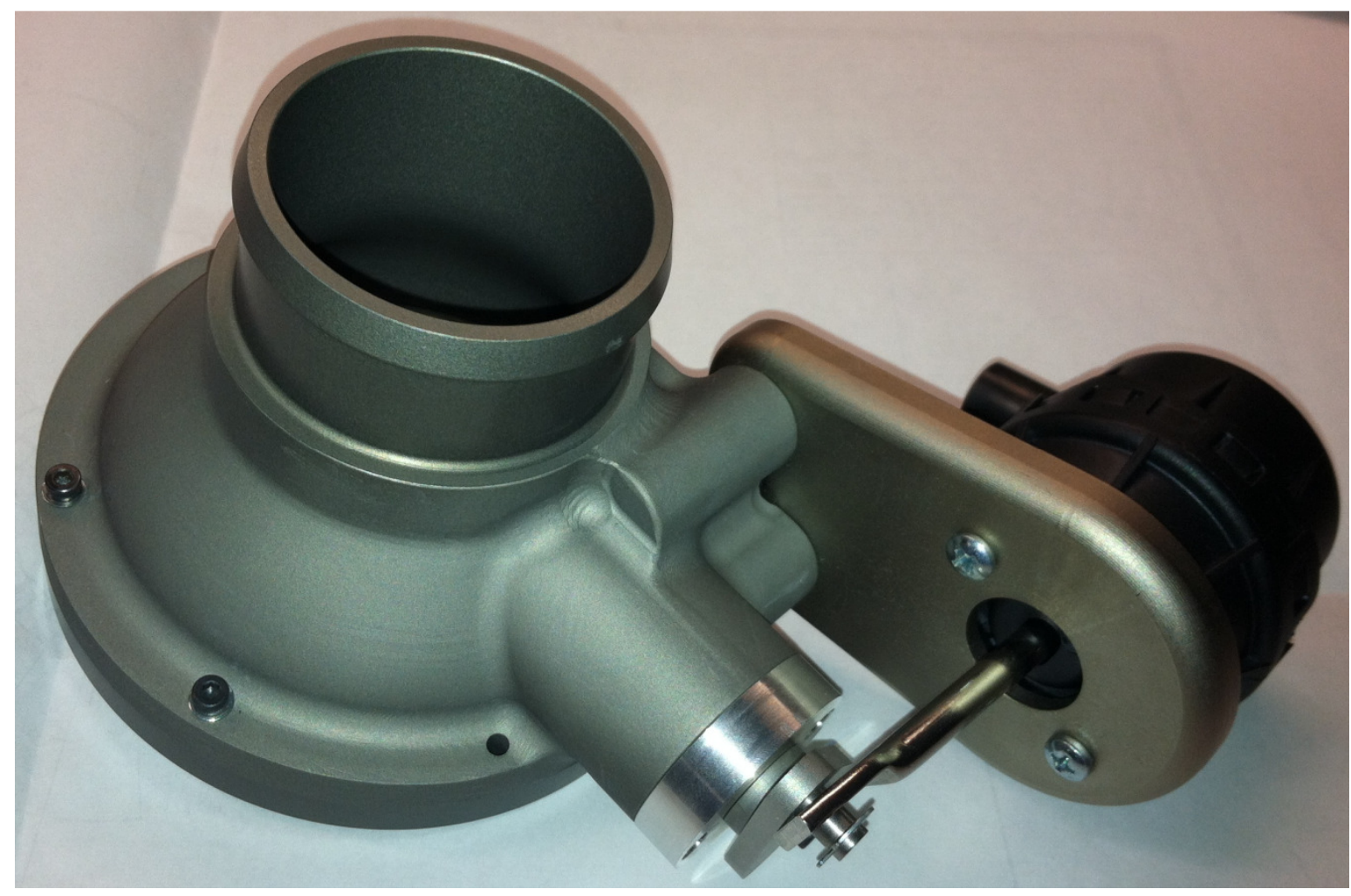

6 Fabrication of prototype advanced MD turbocharger

Fig.5.1 Active casing treatment assembly that is actuated pneumatically by electronic solenoid

Figure 6.1 is the comparison of advanced MD turbocharger (on the left) and a production turbocharger (on the right) that has the similar flow capapcity. Clearly the advanced compressor impeller with arbitrary surface has smaller diameter. So does the advanced mixed flow turbine wheel. That means that for the same flow capacity, the advanced MD may have better transient response and low end performance. For the prototype fabrication, both compressor impeller and turbine wheels are both 5-axis machine milled. The inconel turbine wheel was "electric beam" welded to a steel shaft for better durability (after an initial failure of a prototype single body of shaft and turbine wheel made of total inconel). 


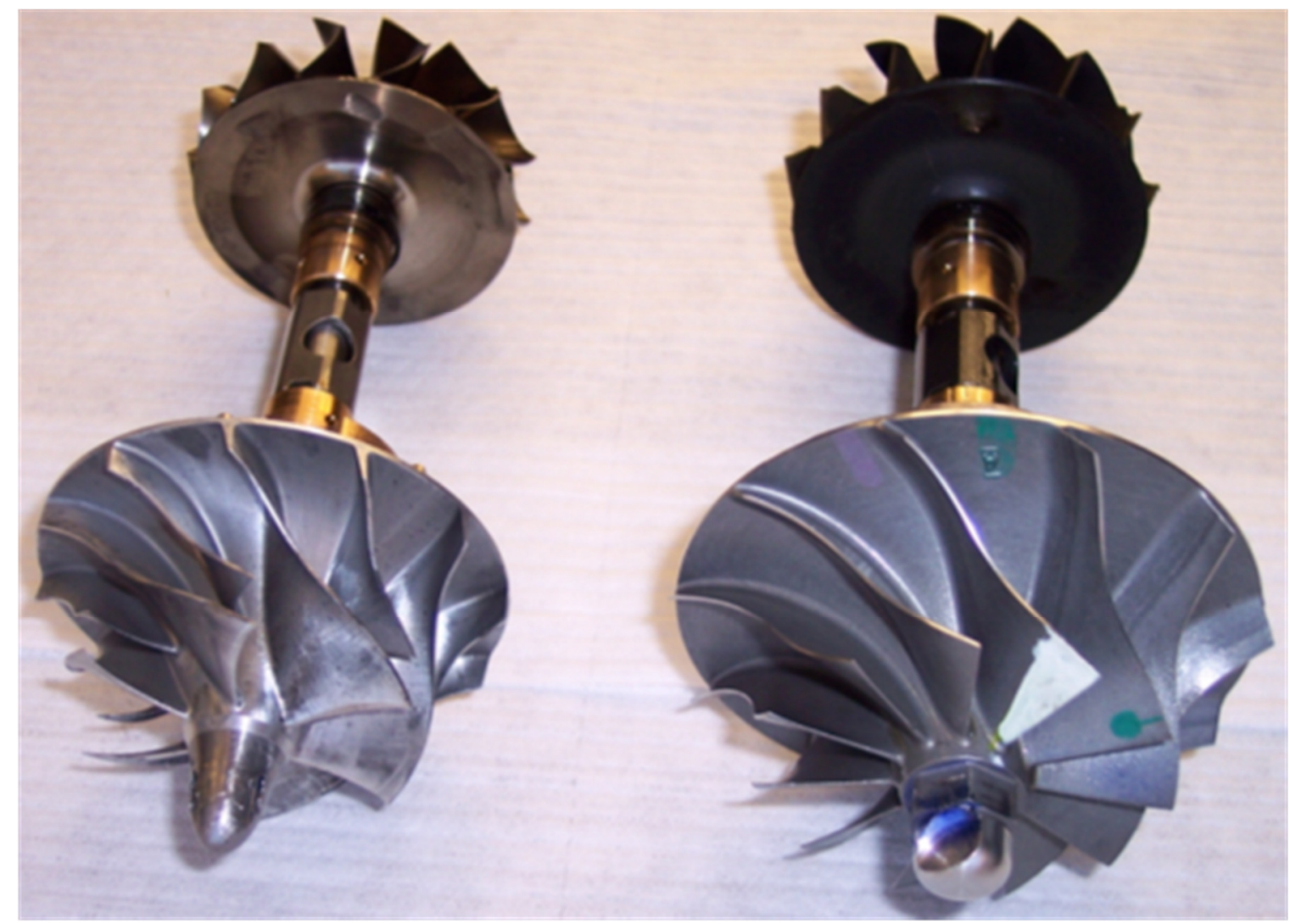

Fig.6.1 Comparison of advanced turbocharger (left) with conventional turbocharger wheels

Figure 6.2 is advanced compressor housing with the ultra-low solidity diffuser vanes (ULSD). The diffuser vanes are five vanes that are individually mounted on the diffusers and are removable. Later on in the study, the advanced aero impeller with active casing treatment with vaneless diffuser was adopted based on the efficiency and operation range. Certainly, the durability concern of the actuation system of ULSD is also a concern.

Different to the moving parts with the actuation system of the active casing treatment (Fig. 5.1 ), the pivotal vanes in the ULSD are exposed to high temperature (crank case ventilation contaminated) air under which coking will be a concern. 


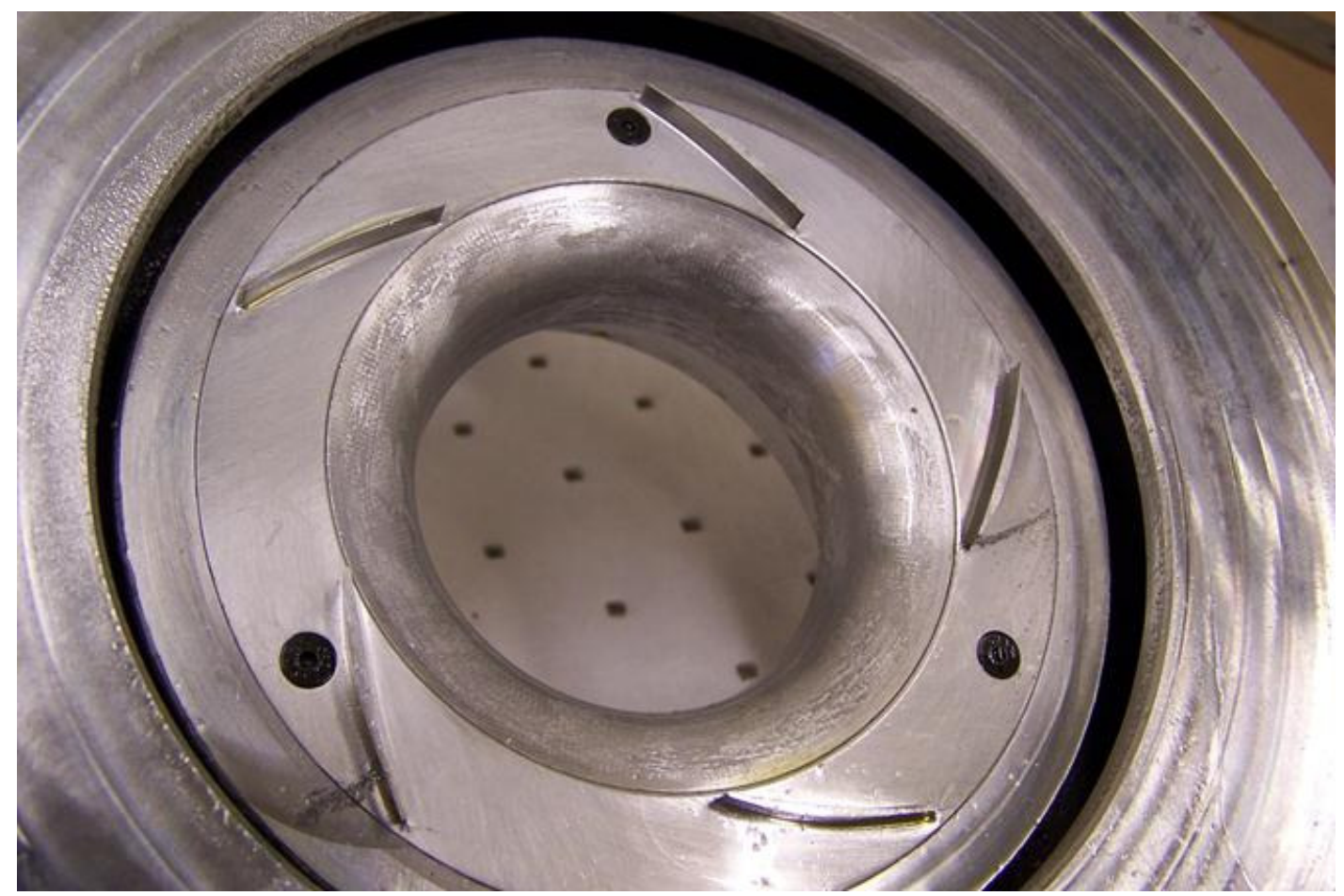

Fig.6.2 Prototype of advanced compressor housing with ultra-low solidity diffuser vane inserts (the vaned diffuser was later on replaced by active casing treatment)

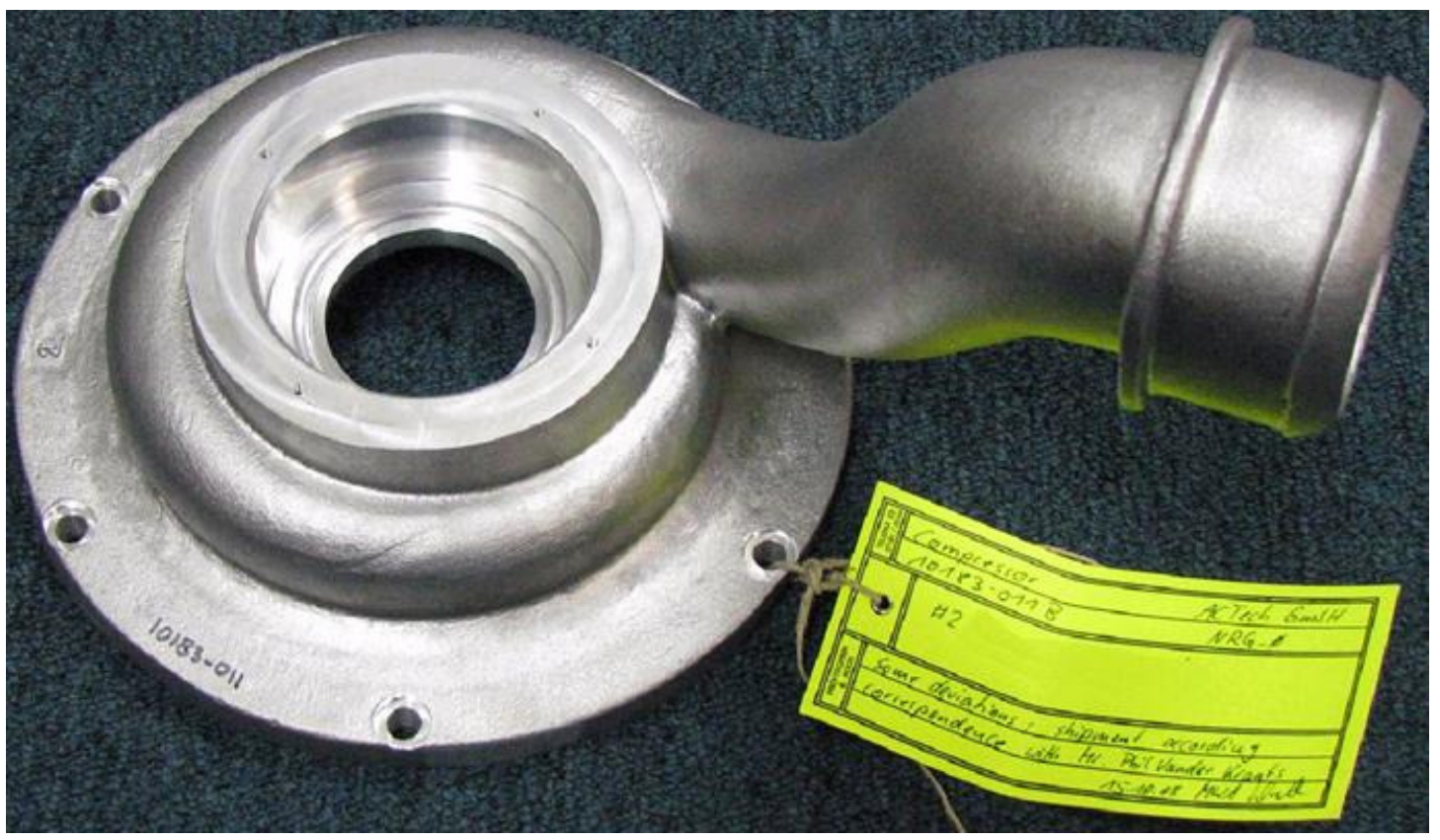

Fig.6.3 Prototype of advanced compressor housing that was matched with a production donor turbocharger (compressor inlet has switchable inserts to evaluate different casing treatment) 


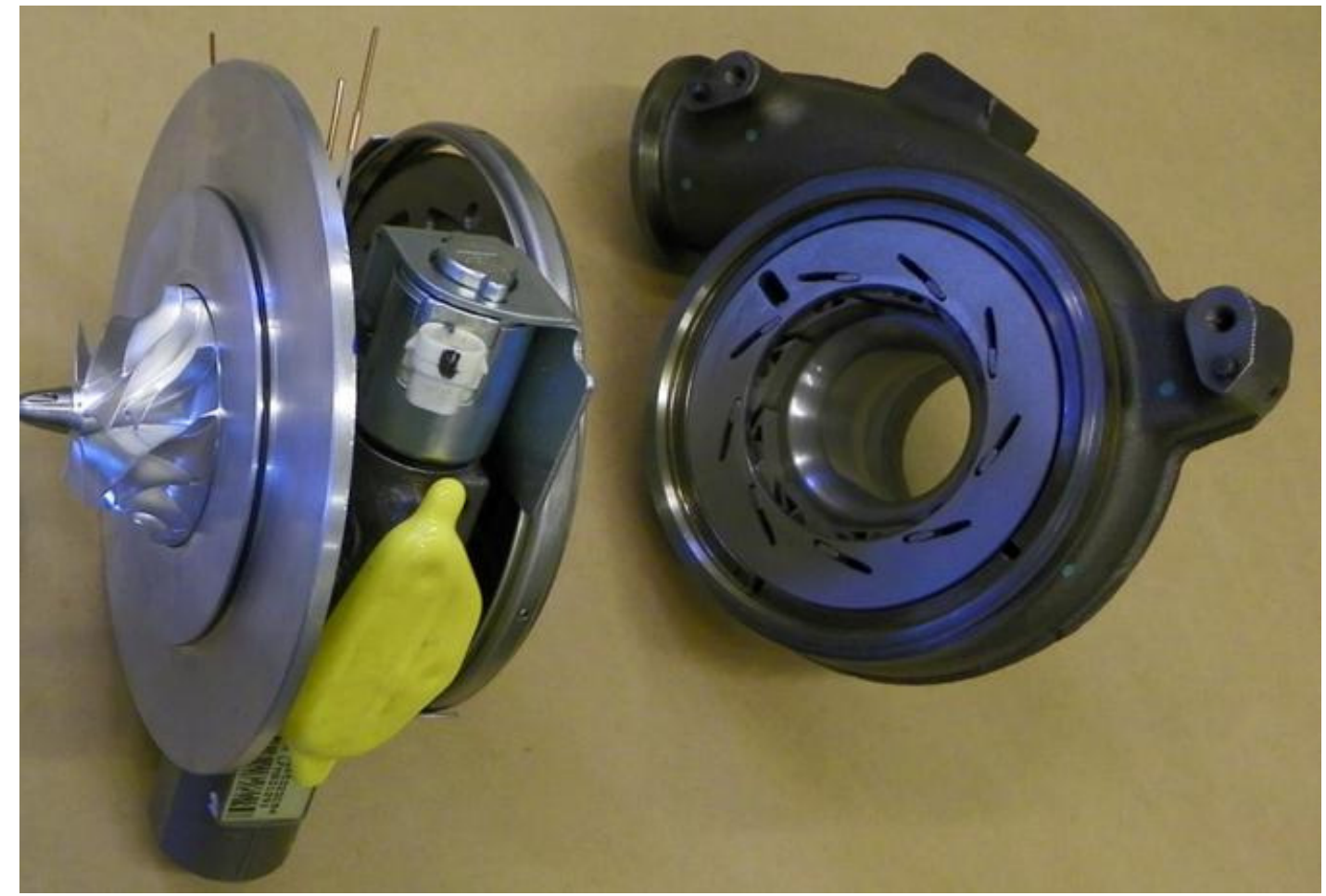

Fig.6.4 Prototype of advanced MD turbocharger assembly

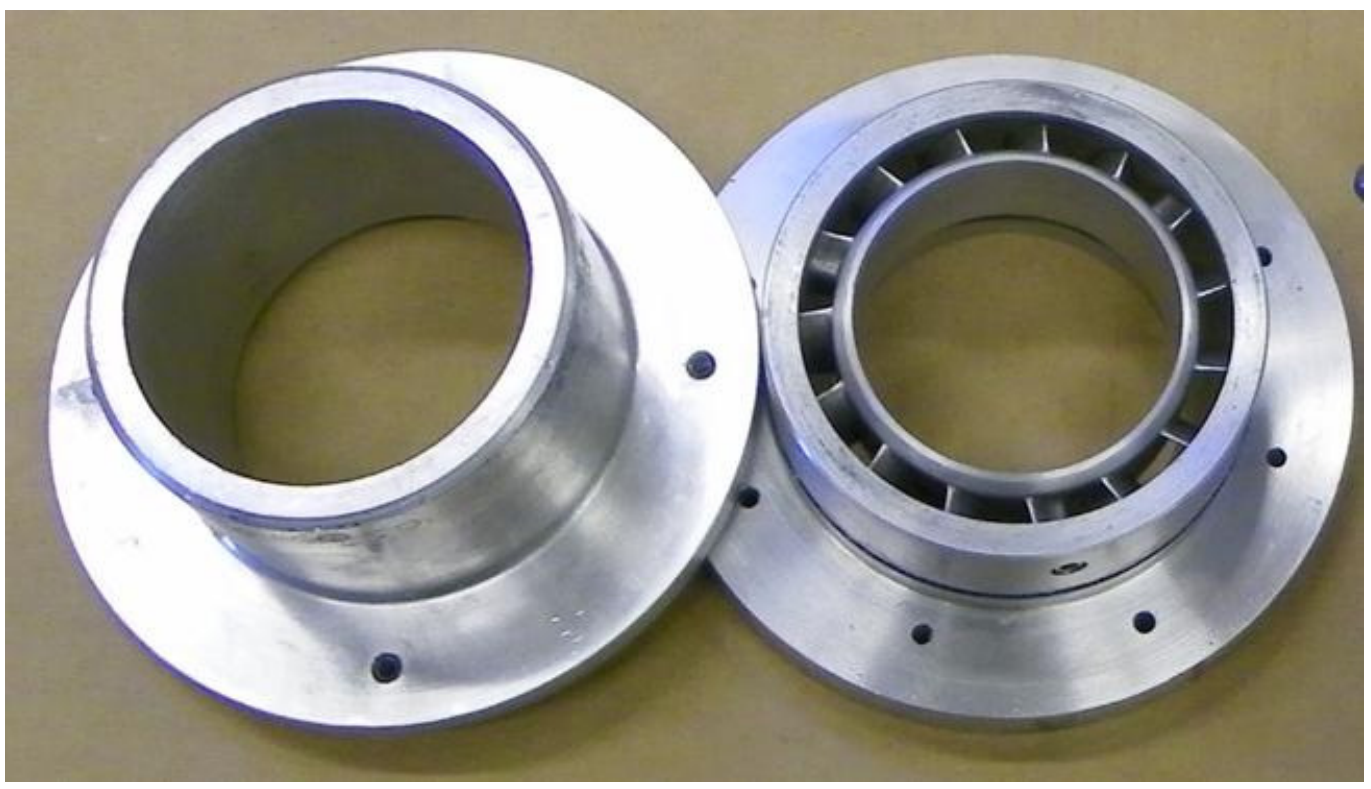

Fig.6.5 compressor insert for different casing treatment configuration 
Steady state flow bench test validation of advanced MD turbocharger performance

\section{1}

Steady state flow bench test of advanced MD compressor impeller

The turbocharger assembly with and without active casing treatment was flow bench tested by a turbocharger supplier. For the flow bench test there was no actuation and control system adopted. Thus the tests were conducted with a modular compressor with switchable inserts for different compressor casing treatment configurations.

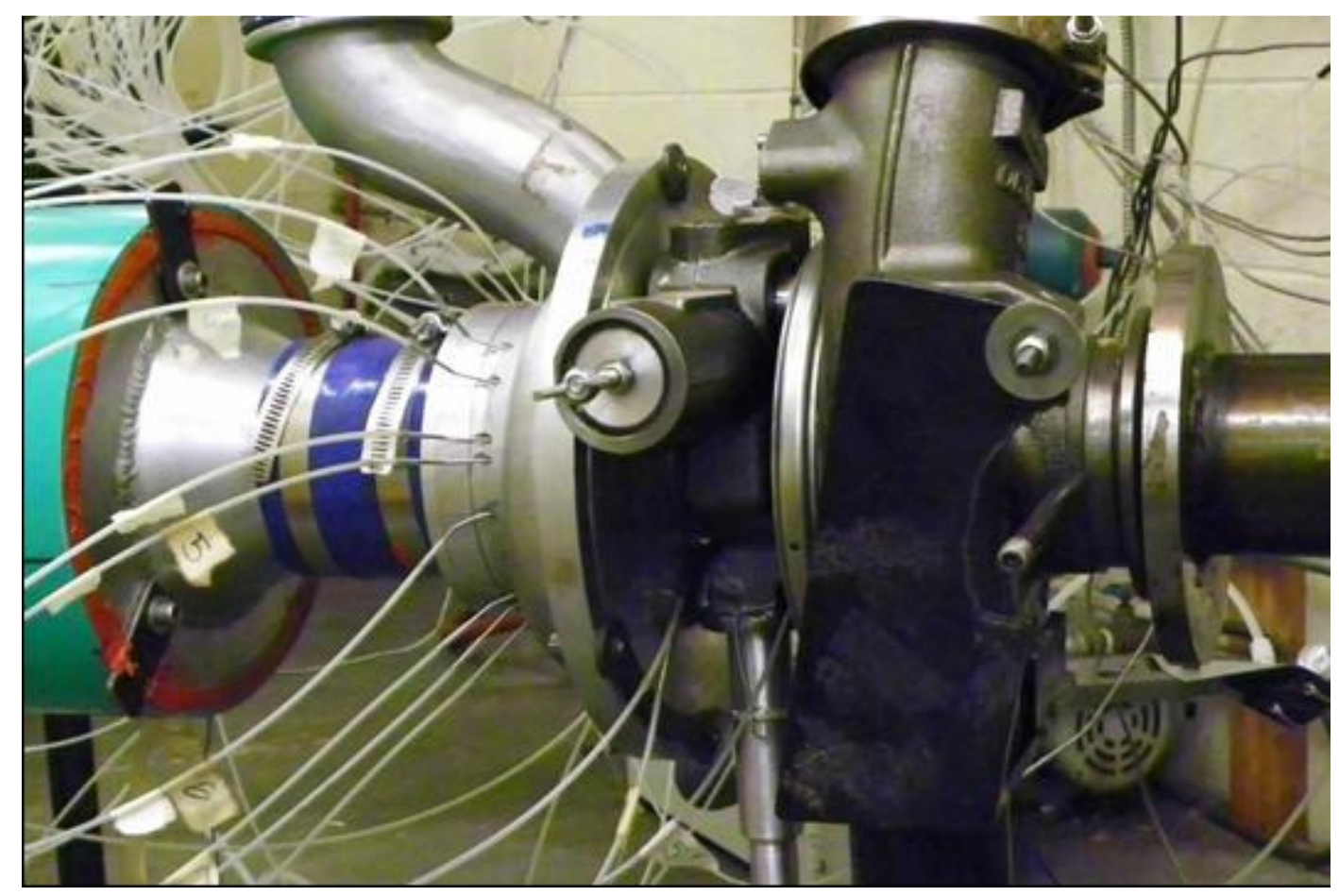

Fig. 7.1 Instrumented advanced MD turbocharger on flow bench

\subsection{Experimental Validation of different casing treatment concepts}

Figure 7.1 shows the compressor performance measurement out of steady state flow bench test at ConceptsNREC which shows that casing treatment $B$ (with guide vanes inside the casing) has surge margin improvement without efficiency and flow capacity penalty, compared with casing treatment option $\mathrm{A}$ (without guide vanes inside the casing). The compressor without casing treatment shows the worst in terms of surge margin. It is worth noting that the aforementioned improvement in surge margin is only limited to pressure ration higher 1.5 .

Finally compressor performance was tested on flow bench to validate the active casing treatment (ACT) concept. Figure 7.2 shows compressor performance comparison between single slot casing treatment option B and dual slot with separate choke slot. From medium to high rotation speed, the ACT extends compressor choke flow rate greatly. The ACT is more effective at high rotation speed, mainly due to the fact that at high speed there will be supersonic flow and strong shock waves inside the compressor where the choke slot control is more useful, as indicated by numerical analysis. 


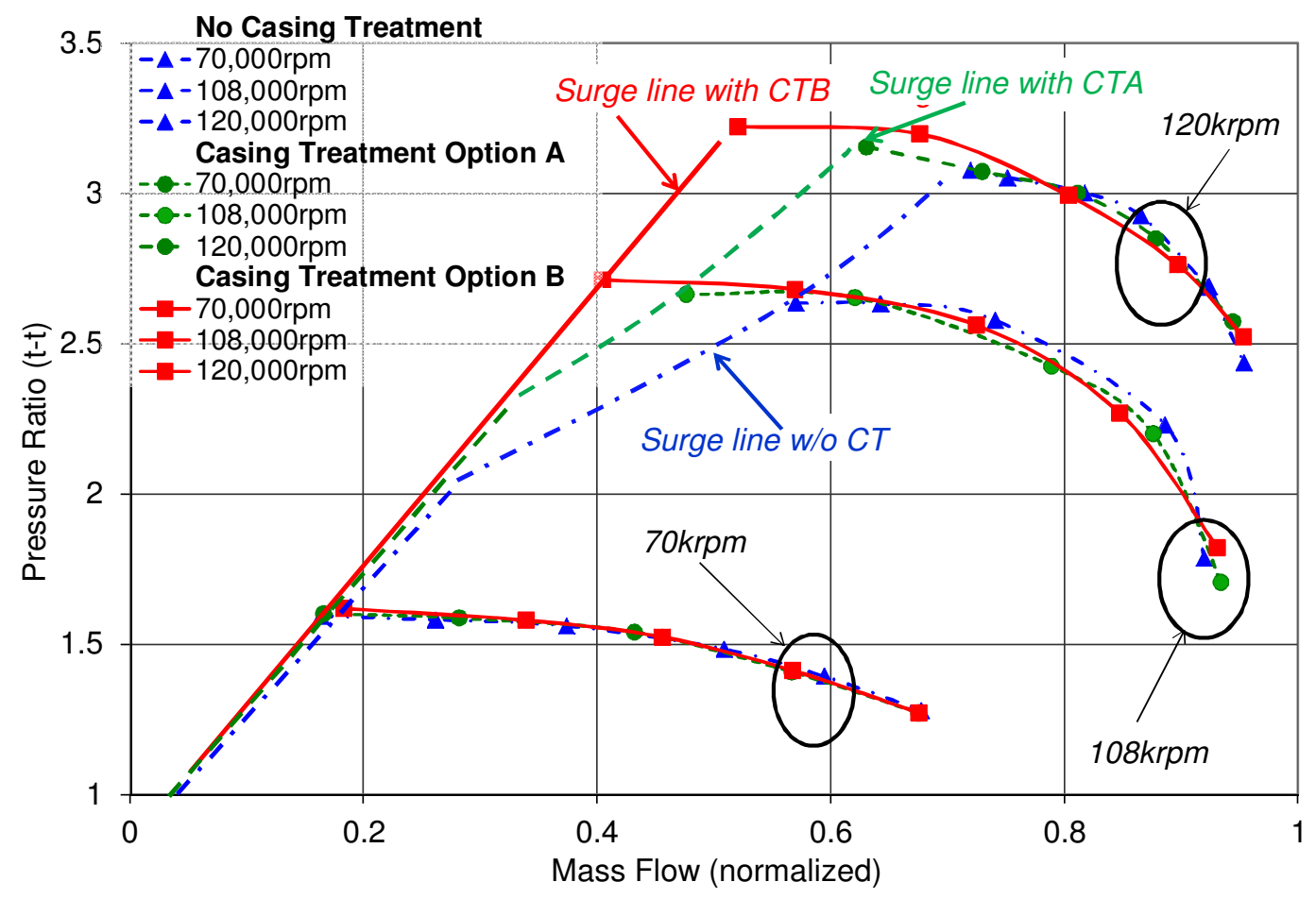

Fig. 7.1a. Flow bench test [49]: Compressor pressure ratio (total to total) vs. mass flow with different casing treatment configurations

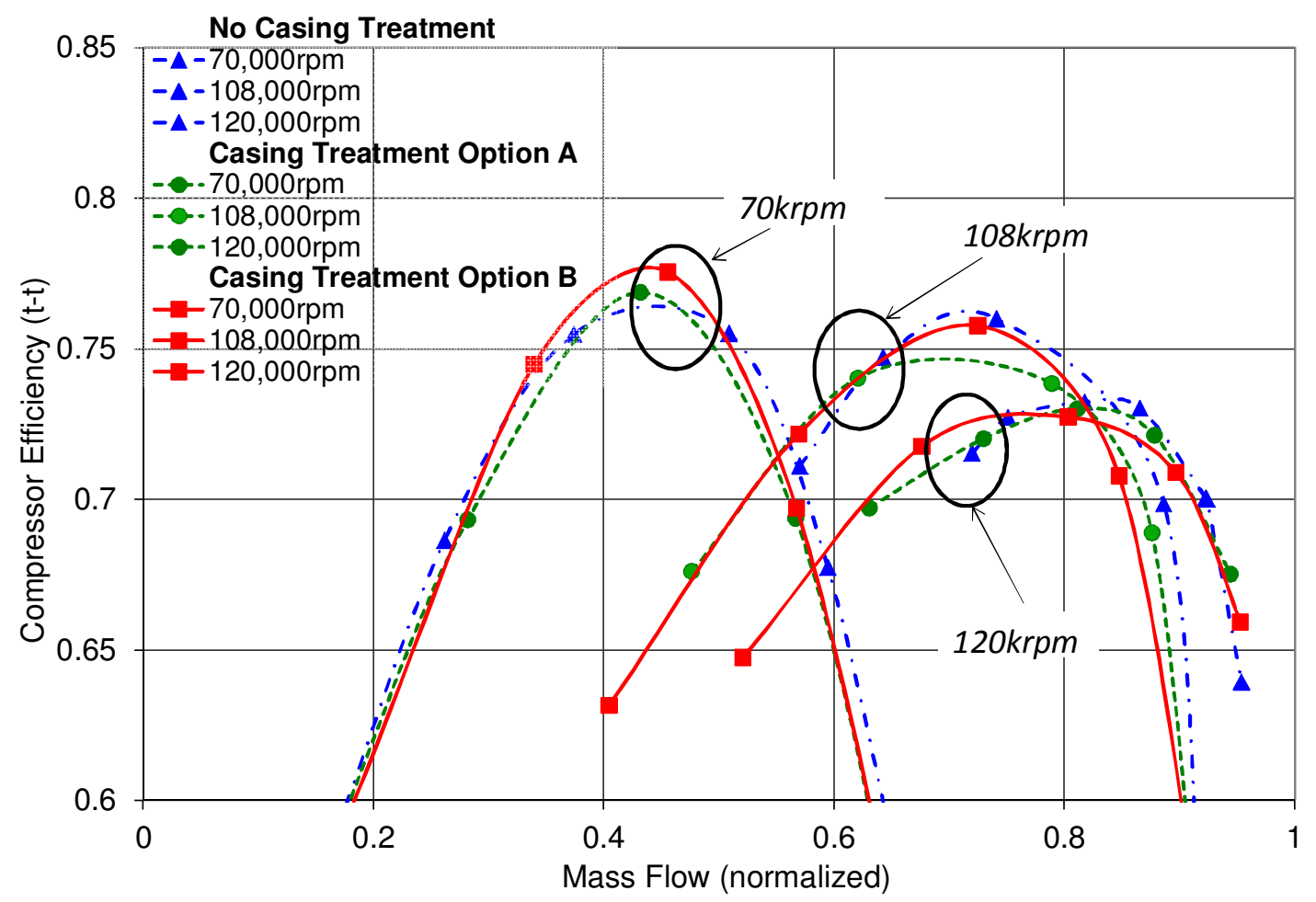

Fig. 7.1b Flow bench test [49]: Compressor efficiency (total to total) vs. mass flow with different casing treatment configurations 
Conventional casing treatment with guide vane (CTB) vs. Active casing treatment (ACT)

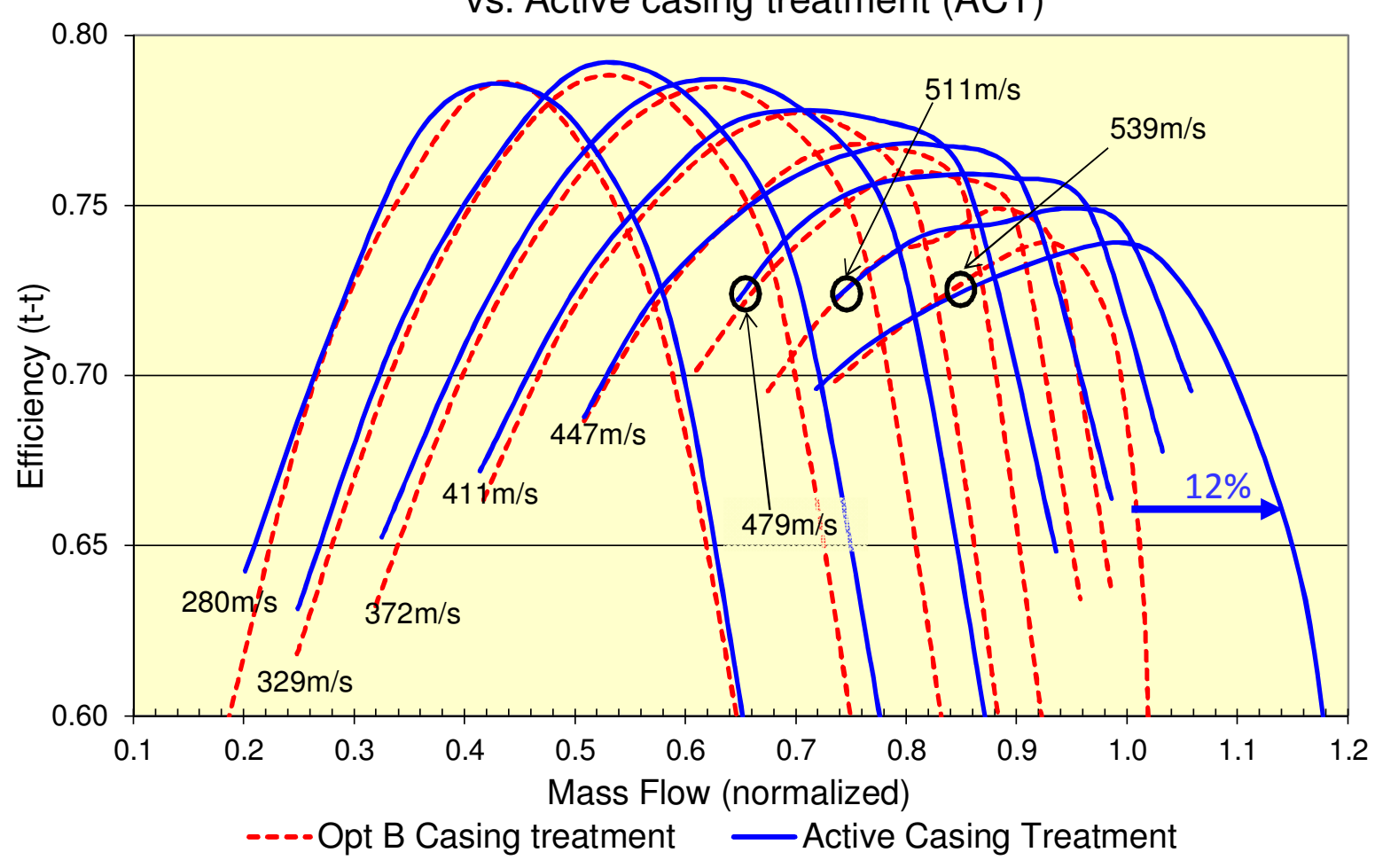

Fig.7.2a. Steady state flow bench test. Compressor efficiency: single-port casing treatment (Opt B, with guide vanes inside) and Dual Port Casing Treatment (w/o guide vanes inside)

The maximum flow capacity of the compressor has been extended by about $15 \%$ at peak rotation speed which is close to the CFD result. If compared at efficiency of $65 \%$ and pressure ratio of 3.0, at which point it will meet the requirement of the engine, there is still about $12 \%$ flow capacity extension. At low-end active SRCT has no efficiency penalty compared to the conventional single slot casing treatment while maintaining the same surge margin, which again verified the numerical result. In summary, the experimental result verified the numerical result and the active casing treatment concept to get a wide flow range compressor with high efficiency.

From the above analysis it can be seen that the active self-recirculation casing treatment casting treatment (SRCT or active casing treatment ACT), consisting of dual bleed slots, named choke and surge slot separately, will combine the performance improvement of the compressor with different SRCT bleed slot configurations. Near choke condition, the choke slot located downstream could be opened to extend the compressor flow capacity; while near surge condition the choke slot needs be closed and the surge slot located in the upstream could be opened to bleed the air out of the impeller passage and extend compressor surge flow margin without efficiency penalty. 
Conventional casing treatment with guide vane (CTB)

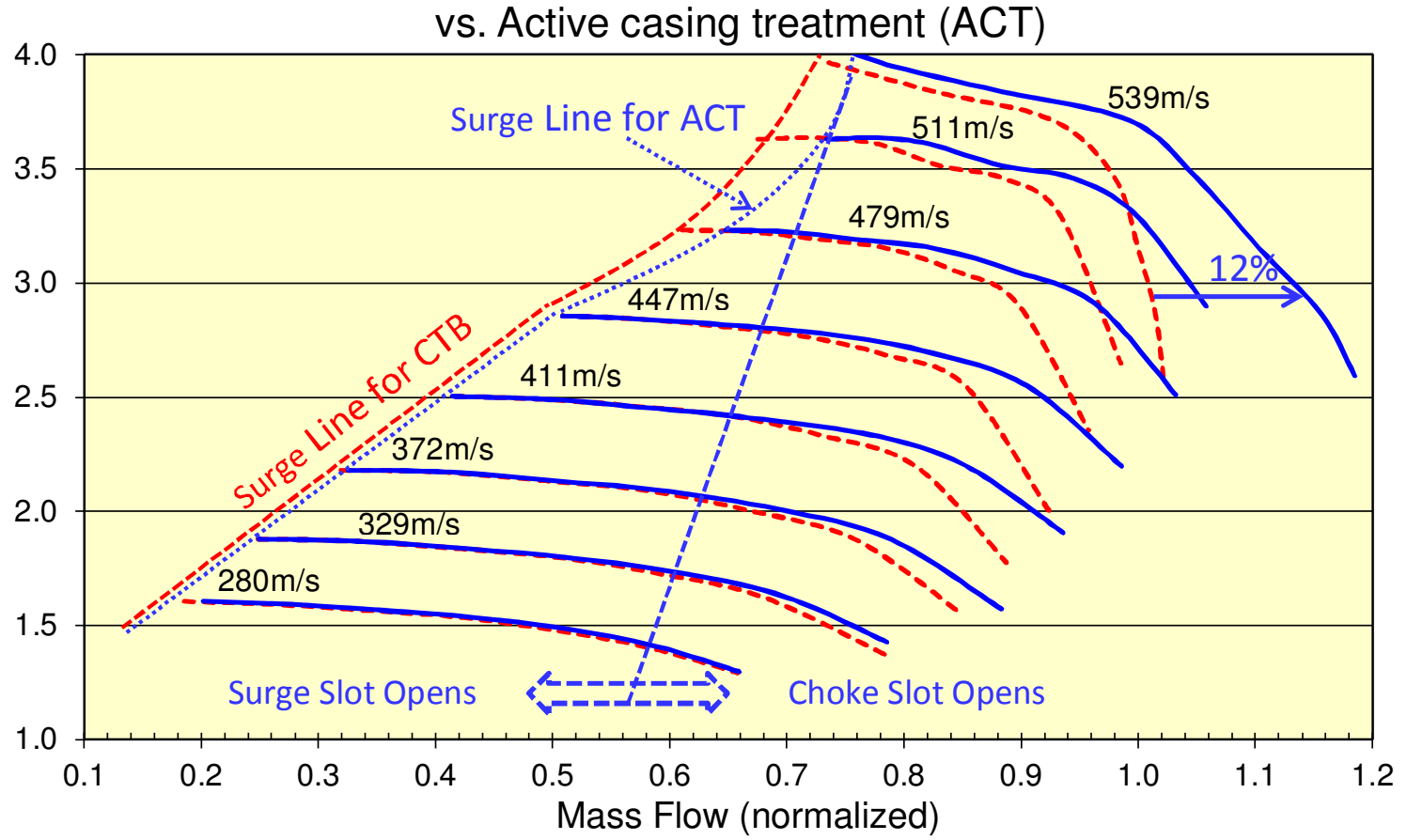

-----Opt B Casing treatment_-Active Casing Treatment

Fig.7.2b. Steady state flow bench test. Compressor pressure ratio: single-port casing treatment (Opt B, with guide vanes inside) and Dual Port Casing Treatment (w/o guide vanes inside)

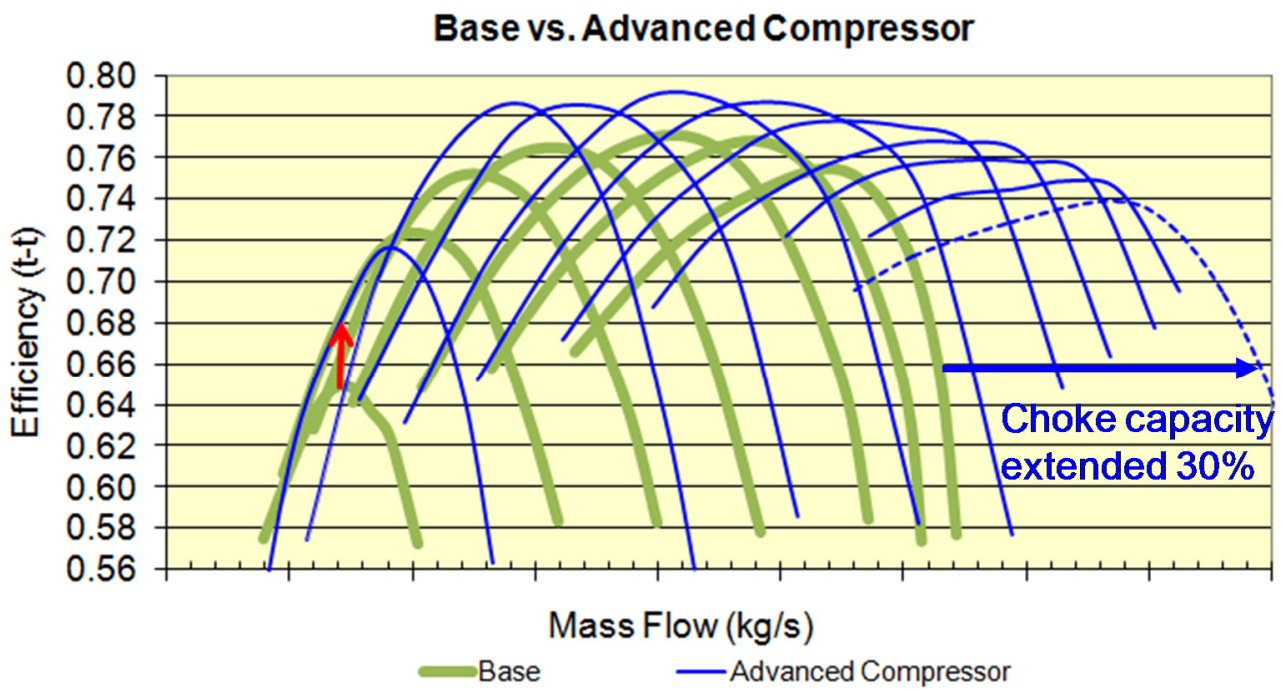

Fig. 7.3 Steady state flow bench test of advanced MD compressor with ACT vs. base compressor 


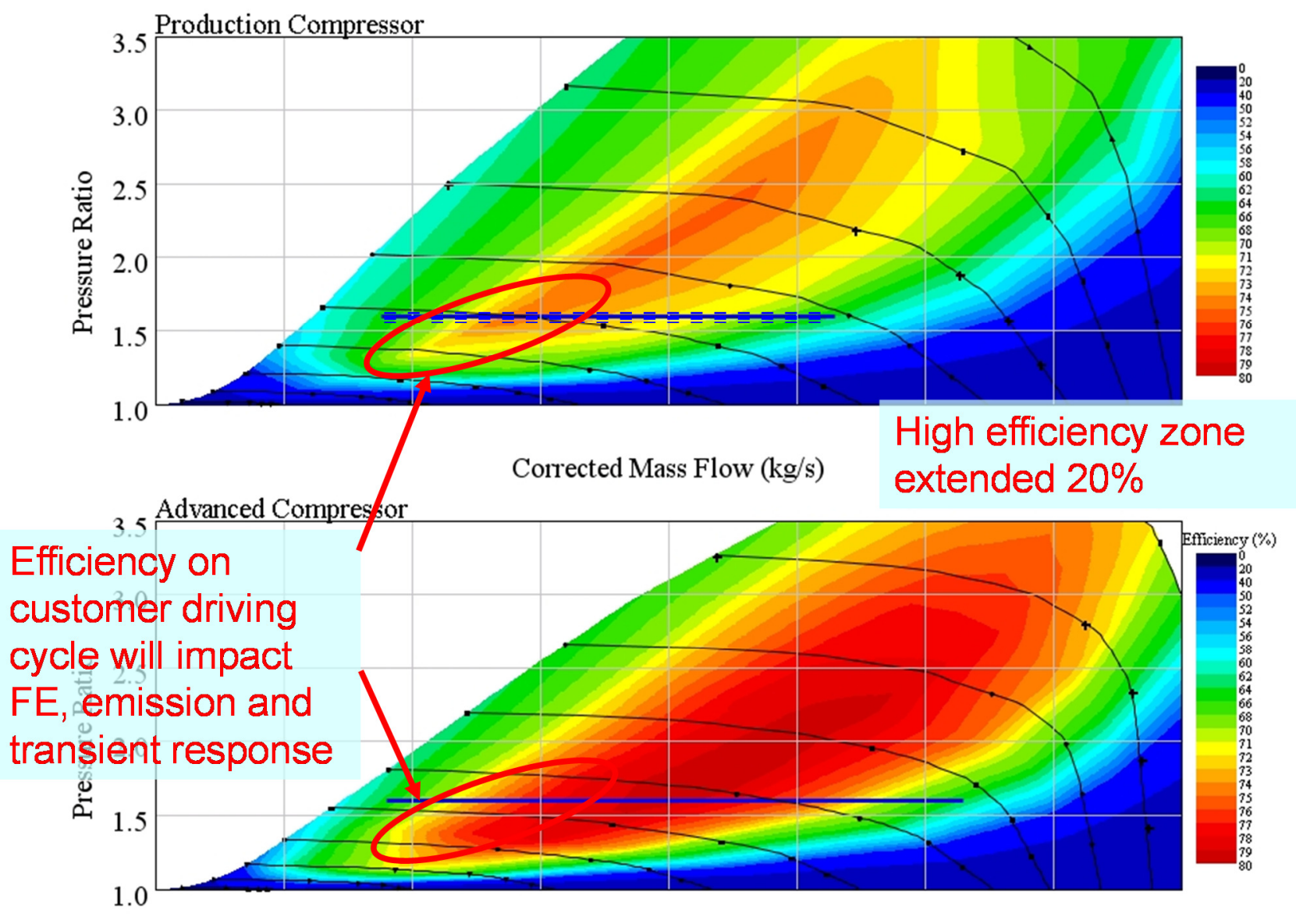

Corrected Mass Flow (kg/s)

Fig.7.4. Steady state flow bench test. Compressor contour plots: donor compressor with conventional single slot casing treatment (top) vs. advanced MD compressor with Dual Port Casing Treatment (w/o guide vanes inside)

Combined with the optimal design of advanced impeller with arbitrary surface by ConceptsNREC, with the intension to deliver superior efficiency over wide operation range, the active casing treatment further extended the flow capacity. Compared with base donor compressor with conventional single slot casing treatment (Fig. 7.3), the advanced MD compressor with arbitrary surface impeller and active casing treatment shows efficiency improvement around $4-5 \%$. But the flow capacity has 30\% enhancement, which is quite significant. The flow capacity enhancement on the advanced MD compressor can be partly attributed to the advanced impeller with arbitrary surface and partly attributed to the active casing treatment (as shown in Fig. 7.2). The two compressor impellers have exactly the same exducer diameter.

The turbocharger assembly with and without dual port casing treatment was flow bench tested by the same turbocharger manufacturer that supplies the donor turbo to ensure transparent test processure. When the surge slot opens at low mass flow area (i.e. the casing treatment A), compared to the Casing Treatment $B$ (with air foil guide vanes inside), the Dual Port Casing Treatment showed an increase in flow capacity (defined by the maximum mass flow at a pressure ratio of 3.0 with $66 \%$ compressor efficiency) of $12 \%$ (Figure 7.2). The benefits of the Dual Port Casing Treatment increase with decreasing pressure ratio for a given turbo wheel speed. As such, the addition of a choke-side slot is 
likely more effective on applications where the typical pressure ratio at the rated power condition is below 3.0 (such as light duty, chassis certified diesel applications and turbocharged gasoline applications). For other cases, such as dynamometer certified Diesel applications, where EGR requirements at full-load conditions push the compressor pressure ratio above 3.5, the addition of a choke-side slot may not provide significant improvement.

The extension of compressor choke flow capacity via dual port casing treatment may also depend, to a large degree, on the design of the impeller, especially the location and blade angle of the splitter blades. As an extension of the conventional casing treatment approach, the optimal design of the dual-port casing treatment and integration with impeller design relies on 3D CFD analyses. To fully exploit this new concept, it is essential to consider the effects of the dual-port casing treatment throughout the entire design process, starting with the impeller conceptual design, which will be a subject of future study.

In the design of conventional compressor impeller, typically the impeller blade passage throat area has to be sized to meet maximum flow capacity requirements. It was well understood [36] that this geometric blade passage throat area has to be further corrected for tip area blockage due to viscous effects, incidence loss, skin friction pressure loss, hub-to-shroud profile distortion, flow mixing and diffusion loss and impeller tip clearance leakage loss, etc. In other words, the geometric blade passage throat area has to be converted into an aerodynamic blade passage throat area which governs the impeller choking flow capacity. It is a common practice to use one or two groups of splitter blades to optimize the trade-off between low end surge and efficiency requirements and high flow capacity requirement, i.e. to have increased blade passage throat area due to fewer blades at the compressor inlet. Noticeably, the introduction of splitter blades creates extra geometric and aerodynamic blockages along the impeller flow passage.

Results indicate that this concept have the potential to rebalance the compressor impeller design focus more towards the low flow region for improved customer drivingcycle performance without sacrificing the rated power.

Introducing a separate injection slot downstream of the shock wave induced flow passage blockage can take advantage of the expansion following the shock wave near splitter blades thus effectively enhancing choke flow capacity without compromising performance at low end. The steady state flow bench test demonstrated that the dual port casing treatment can extend the flow capacity by $12 \%$ without compromising low end efficiency and surge performance of the compressor.

Mixed flow turbine has long been recognized that can shift the high efficiency zone to low $U / C$ area, even though the peak efficiency area may not necessarily be higher than a radial flow turbine. The intention at the time was focused on better utilization of pulsation energy out of engine exhaust, i.e. the instantaneous high pressure pushes operation point of the turbine into low $\mathrm{U} / \mathrm{C}$ area where mixed flow turbine has been demonstrated outperforming conventional radial flow turbine. The benefit of mixed flow turbine under pulsation flow was mostly recognized in academia since most turbo suppliers quantify turbine performance out of a turbocharger assembly on a steady state flow bench, i.e. the turbine $\mathrm{U} / \mathrm{C}$ measurement is limited to a very small area dictated by compressor stable operation range. When Ford started this advanced turbocharger research in 2007 with 
ConceptsNREC, the mixed flow turbine was selected as a key technology for a different reason, i.e. heavy EGR required for future NOx emission compliance also requires operating with high expansion ratio, thus low $\mathrm{U} / \mathrm{C}$ for extended of time.

The mixed flow turbine is characterized by a non-radial inlet that reduces the flow path curvature and effectively reduces the secondary flow losses. The other noticeable feature is that the radial flow turbine has the inducer blade angle parallel to the rotational axis while the mixed flow turbine typically has inducer blade angle round 20-50 deg relative to rotational axis which helps the inducer to adapt to different instantaneous incidence angle of incoming exhaust flow that would vary with the flow rate and, more importantly, the pulsed pressure. Figure 7.5 is a GTPower simulation of instantaneous turbine speed ratio $(\mathrm{U} / \mathrm{C})$ vs. crank angle at a part load engine condition (6 bar BMEP@1500rpm) with high level of high pressure EGR for US 2010 NOx emission compliance. Apparently turbine operates significant portion of the cycle with $\mathrm{U} / \mathrm{C}$ below 0.375 , where the mixed flow turbine would outperform the conventional radial flow turbines.

The design details of the advanced mixed flow turbine are highlighted in section 3.2. Figure 7.6 is the comparison of steady state flow bench performance of the advanced mixed flow turbine vs. the conventional radial (base) turbine. The flow bench test data shows that the advanced mixed flow turbine has slightly improved peak turbine efficiency, especially at VGT partial open positions. But most importantly the mixed flow turbine pushes peak efficiency towards low $\mathrm{U} / \mathrm{C}$ area, i.e. the mixed turbine would operate in much higher efficiency in low $\mathrm{U} / \mathrm{C}$ areas, compared to the conventional radial flow base turbine. Unfortunately, the steady state flow bench limits the experimental range in a very narrow area of $\mathrm{U} / \mathrm{C}$.

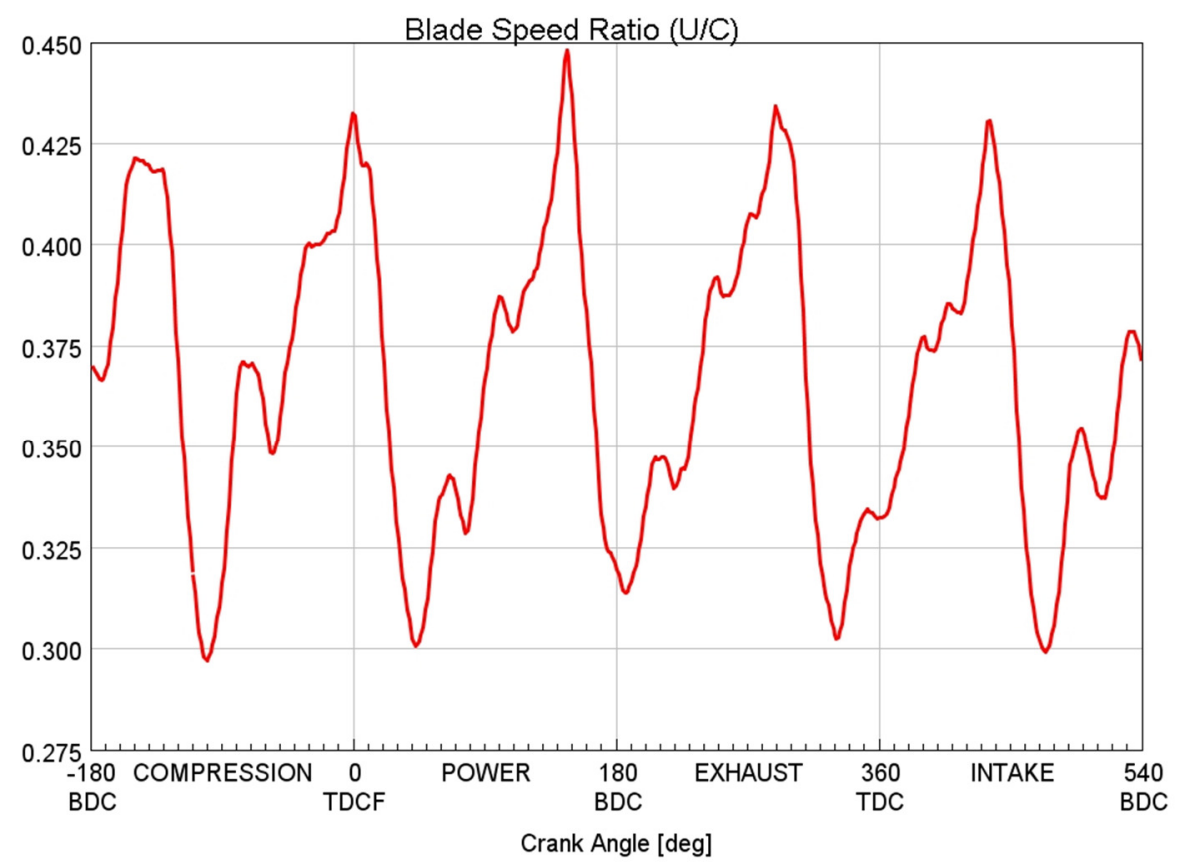

Fig. 7.5 Turbine speed ratio U/C varies during one cycle of a four stroke I5 diesel engine (GTPower simulation) 

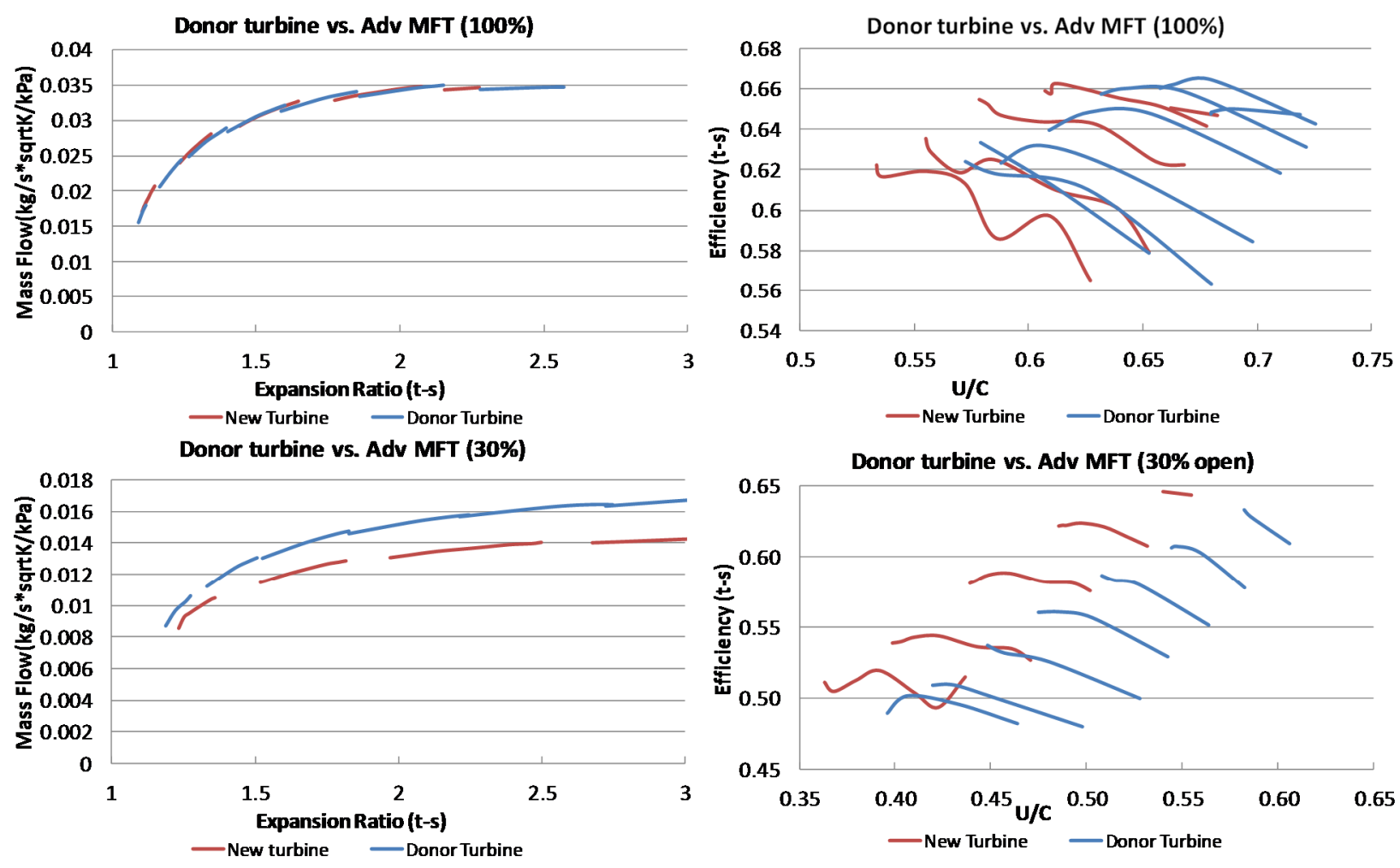

Fig. 7.6 Comparison of steady state flow bench test: the new advanced mixed flow turbine vs. radial flow donor turbine.

\section{Steady state MD diesel engine performance test with advanced MD turbocharger}

Due to heavy EGR and pulsed flow into the turbine, a turbine may operate significant portion of the time in $\mathrm{U} / \mathrm{C}$ areas that are not typically covered by steady state turbocharger flow bench. Also many light load and low engine speed operation points would have the compressor operate in speeds that are not typically covered by steady state compressor maps. The engine dynamometer test would be necessary to validate the overall engine performance enhancement of the advanced turbocharger. For that purpose, a back to back engine validation test with base donor turbocharger and the advanced turbocharger (including the advanced impeller with arbitrary surface, ACT and mixed flow turbine) was conducted on a 6.7L diesel engine. The base engine was calibrated for the 2010 US emission level. Only the feedgas gaseous emissions were measured.

A part load point, 3 bar BMEP at $1250 \mathrm{rpm}$, was selected where the city cycle is heavily weighted. This part load point was also used as one of the design target when the compressor and turbine was designed since the turbo operates at the low end of the range that could be tested on a turbocharger flow bench and is still in the center of a typical city driving cycle. Figure 6 highlights the performance comparison between the newly developed advanced turbocharger and the base donor turbo. The test data shows that at this part load condition, at the same feedgas NOx and smoke level as those in the $2010 \mathrm{MY}$ calibration, BSFC has 3\% improvement (top and bottom left), which is consistent with the reduction in turbo delta pressure (turbine inlet pressure - compressor out pressure, top right), or pumping loss, as well as the improvement in compressor efficiency (bottom right); when the NOx level was further reduced via the increase in EGR, the new 
turbo shows more BSFC benefit. It should be noted that since two sets of data (between the new and donor turbo) were not compared exactly at the same smoke level, the discrepancies between air fuel ratio and variable geometry turbine calibration make it difficult to build a direct correlation between the BSFC reduction with pumping loss and compressor efficiency improvement for different level of NOx. But, since the pair of points that showed 3\% BSFC improvement have much lower smoke with new advanced turbo, it is safe to claim that the new turbo improves the BSFC by $3 \%$.

Feedgas NOx vs. BSFC

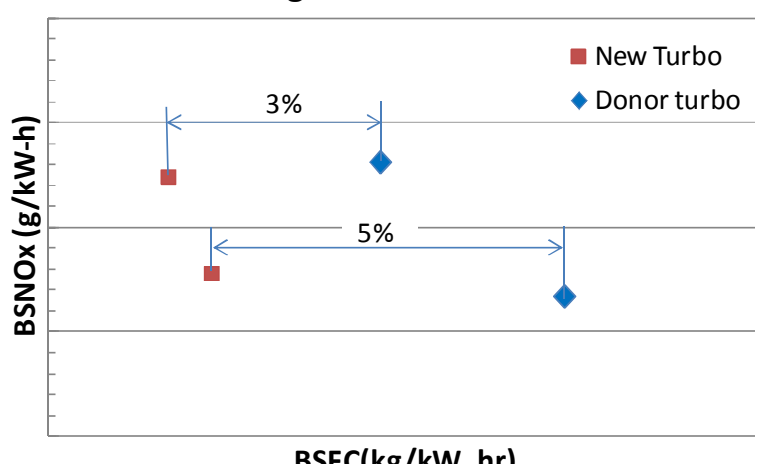

Smoke vs. BSFC

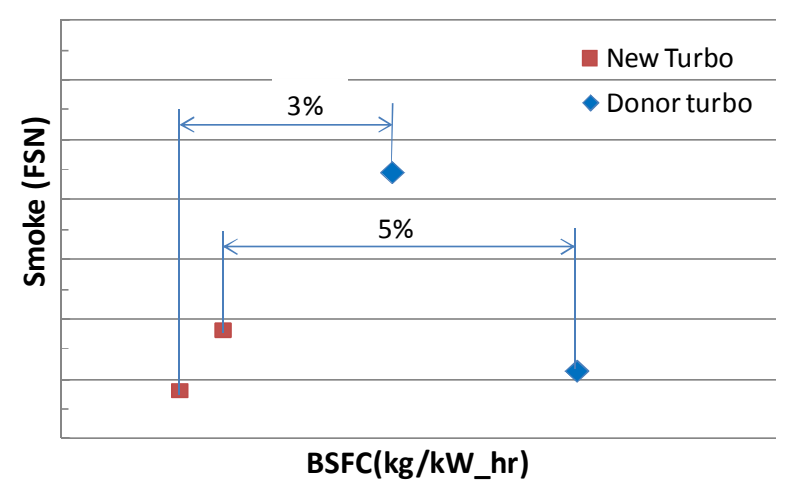

Feedgas NOx vs. Turbo Delta P

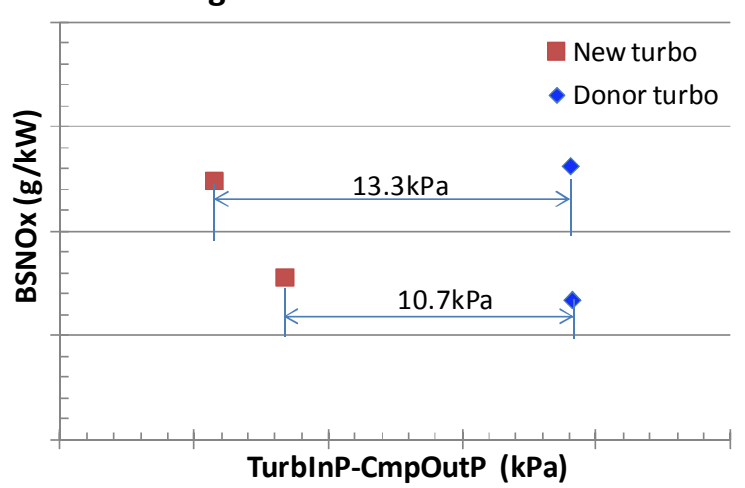

Smoke vs. Compressor Efficiency

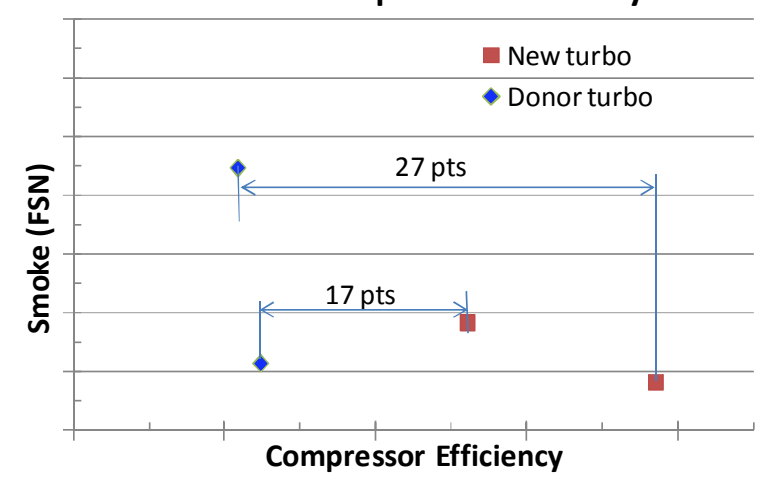

Fig. 8.1 Part load steady state MD engine performances at 3 bar BMEP@1250rpm: advanced MD turbo vs. donor turbo

All efficiency improvement in turbocharger (e.g. compressor, turbine and bearing improvement) will translate into lower pressure differential between turbine inlet pressure and compressor outlet pressure, i.e. lower pumping loss on the engine (as shown on top right of Fig. 8.1)

When the engine operates at full load, the efficiency improvement in the turbocharger will be represented by higher torque, and/or lower turbine inlet temperature, as shown in Fig. 8.2. The steady state data indicated that the engine with the new advanced turbocharger, including advanced impeller with arbitrary surface, active casing treatment, has demonstrated $30 \%$ more power at rated condition, and with $35 \mathrm{deg} \mathrm{C}$ 
reduction in turbine inlet temperature, which can be attributed to 11 percentage point improvement in compressor efficiency, and 5 percentage point improvement in turbine efficiency at rated condition, compared to the base conventional donor turbocharger. Similar performance improvement can be observed at full load with different engine speed, even though the efficiency improvement distribution between compressor and turbine varies at different operation conditions, largely due to the variable geometry turbocharger, EGR and AFR calibration. During the engine full load tests, the engine cylinder pressure limit and turbo speed limit were kept at the same level.
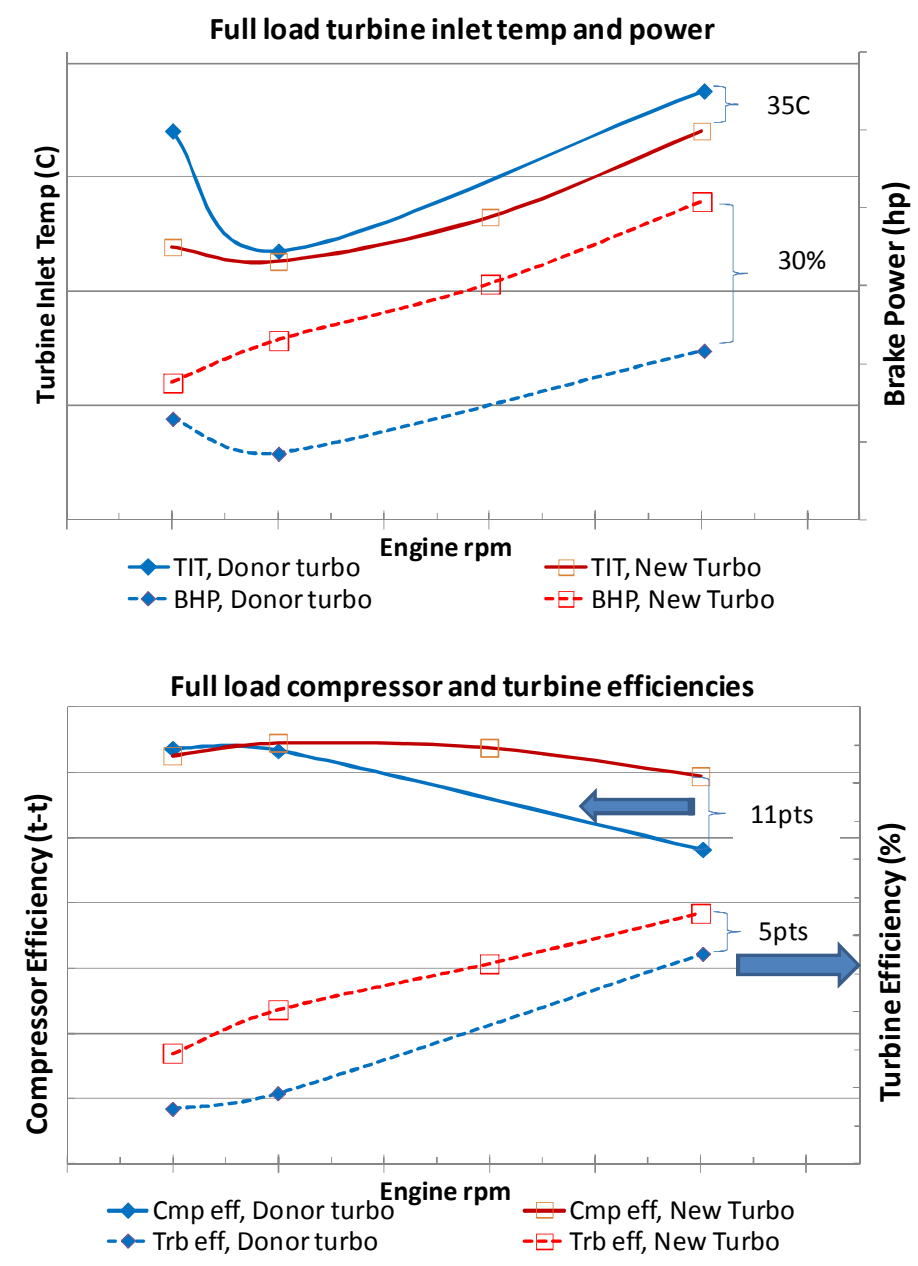

Fig. 8.2 Comparison of steady state full load engine performance: the new advanced MD turbocharger vs. conventional donor turbocharger.

9 Advanced turbocharger development for light duty diesel application

To promote light duty diesel applications for light duty vehicles with better fuel consumption and mitigate the technical accomplishment that was achieved into LD diesel applications, the second phase of the turbocharger research program focused on an 
advanced LD turbocharger development for light duty diesel application for fuel economy improvement at Tier II Bin 5 emission level..

A production 3.2L 15 diesel engine was selected as donor engine. Different to MD or HD diesel applications, the LD diesel typically is more cost sensitive. Therefore certain design changes have to be made or innovative technologies have to be investigated for low manufacturing cost for the advanced LD turbochargers, i.e. the LD turbocharger is not a scale-down version of the advanced MD turbocharger.

One particular area of change is the compressor impeller. The advanced impeller of MD compressor used arbitrary surface, which is package space effective, highly efficient but more expensive. It was estimated by various turbocharger manufacturers that the mill cost for arbitrary surface blade can be anywhere $30-100 \%$ more expensive. Thus the primary assumption for advanced LD compressor is ruled surface blades.

Table 9.1 highlighted the design target for the advanced LD turbocharger that was determined necessary via GTPower simulation to achieve the engine fuel economy savings on customer driving cycles. The main focus of the program is to improve the fuel economy at customer driving cycles, i.e. light low and low to medium engine speed, which is represented by match point " $\mathrm{D}$ ", while stretching the " $\mathrm{C}$ " in mass flow for high engine brake power.

\begin{tabular}{|c|c|c|c|c|c|}
\hline $\begin{array}{c}\text { Comprs } \\
\text { match }\end{array}$ & Priority & $\begin{array}{c}\text { Corrected } \\
\text { Mass Flow }\end{array}$ & $\begin{array}{c}\text { Press } \\
\text { Ratio }\end{array}$ & $\begin{array}{c}\text { Prod. Cmp } \\
\text { Efficiency }\end{array}$ & $\begin{array}{c}\text { Propsd Cmp } \\
\text { Efficiency }\end{array}$ \\
\cline { 2 - 6 } & $\mathbf{k g} / \mathbf{s}$ & $\mathbf{t}$-t & t-t & t-t \\
\hline A & $\mathbf{2}$ & 0.042 & 1.55 & $57.6 \%$ & $66.0 \%$ \\
\hline B & $\mathbf{3}$ & 0.112 & 2.72 & $67.3 \%$ & $69.0 \%$ \\
\hline C & $\mathbf{1}$ & 0.241 & 2.90 & $74.9 \%$ & $70.0 \%$ \\
\hline D & $\mathbf{1}$ & 0.064 & 1.47 & $66.4 \%$ & $77.0 \%$ \\
\hline
\end{tabular}

\begin{tabular}{|c|c|c|c|c|c|}
\hline $\begin{array}{c}\text { Turbine } \\
\text { match }\end{array}$ & Priority & $\begin{array}{c}\text { Reduced } \\
\text { Mass Flow }\end{array}$ & $\begin{array}{c}\text { Expansion } \\
\text { Ratio }\end{array}$ & $\begin{array}{c}\text { Prod Turb } \\
\text { Efficiency }\end{array}$ & $\begin{array}{c}\text { Propsd turb } \\
\text { Efficiency }\end{array}$ \\
\cline { 2 - 6 } Point & $\mathbf{k g} /$ s-sqrtK/kPa & t-s & t-s & t-s \\
\hline A & $\mathbf{2}$ & 0.006 & 2.00 & $52.0 \%$ & $51.0 \%$ \\
\hline B & $\mathbf{3}$ & 0.011 & 2.76 & $63.0 \%$ & $64.0 \%$ \\
\hline C & $\mathbf{1}$ & 0.019 & 2.23 & $63.0 \%$ & $65.0 \%$ \\
\hline D & $\mathbf{1}$ & 0.010 & 1.61 & $57.0 \%$ & $65.0 \%$ \\
\hline
\end{tabular}

Table 9.1 Performance target for advanced LD turbocharger for a LD diesel application

9.1

LD turbocharger development target

The compressor impeller with ruled surface can also be designed for better low end efficiency, typically with reduced flow capacity. To address the need for high flow capacity and pressure, the impeller diameter has to increase. A production IHI (Ishikawajima Harima Heavy Industries) turbocharger was selected as the base architecture for the turbocharger design with optimizations focused on compressor impeller and turbine wheel designs. As mentioned above, the LD compressor has to be 
ruled surface for lower manufacturing cost, which is also possible to designed such that the low end compressor see improvement while having the active casing treatment extend the flow capacity.

An advanced impeller design with ruled surface but large blade angle was used on the compressor side to improve the efficiency and surge margin at low mass flow areas of the compressor map, allowing greater EGR flow while extending the flow capacity by using active casing treatment on compressor cover.

Mixed flow turbine technology was used on the turbine side due to its performance characteristics, particularly high efficiency at low speed ratios relative to the base conventional radial flow turbine. This characteristic is relevant to increased EGR operation required for future diesel applications.

Both steady state and transient engine dynamometer testing of FTP transient cycles at Tier II Bin 5 emission level show that the advanced turbocharger technology enables around $3 \%$ fuel economy improvement on the engine while meeting the same emissions level.

\section{2}

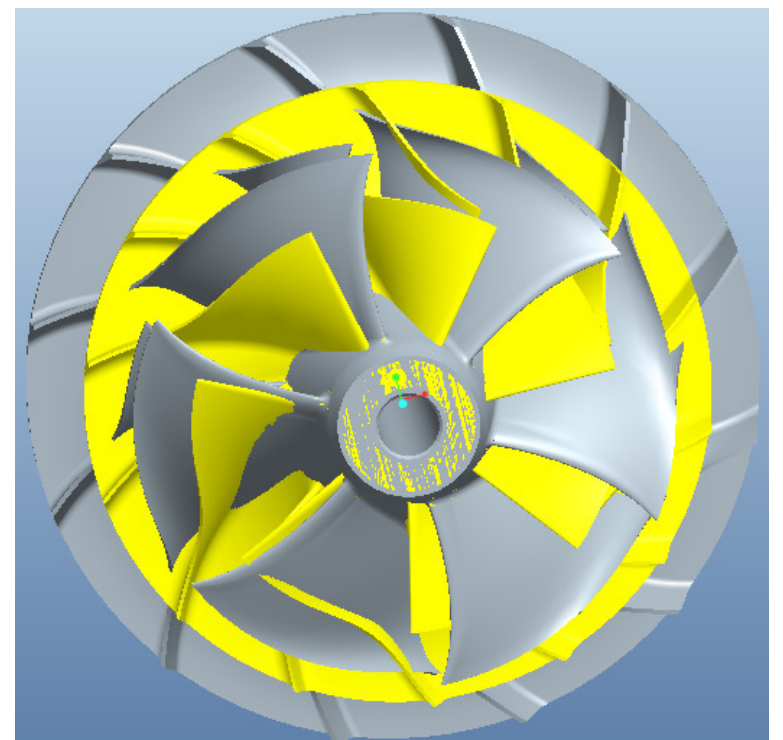

Fig. 9.1 Comparison of advanced MD (grey) and LD (yellow) compressor impellers
The advanced MD compressor impeller has arbitrary surface that requires certain level of point milling thus higher manufacturing cost. Since light duty diesel more cost sensitive, a more cost effective impeller design concept is used, i.e. ruled surface. Figure 9.1 highlights the difference of MD compressor impeller with arbitrary surface and LD compressor impeller with ruled surface. The LD compressor with the ruled surface, when designed for optimal low end efficiency, seems to be relatively large, compared with the the MD compressor impeller with arbitrary surface, since it has only $63 \%$ of the mass flow capacity of the MD compressor. Therefore the LD compressor impeller is less compact and requires a large size turbine.

The aforementioned active casing treatment allows the design to be focused

on low end efficiency improvement and more surge margin. A conventional impeller with ruled surface is typically represented by the relatively small blade angle (relative to rotational axis) at the impeller inlet (Fig. 9.2). As illustrated by Fig. 9.2, at low flow conditions, flow separation (Fig. 9.2b) will occur at the leading edge of impeller when the mass flow rate is low, potentially leading to efficiency loss and the onset of stall or surge. The advanced LD impeller uses a large blade angle at the impeller leading edge (for both full and splitter blades) that better matches relative velocity of the air when the mass flow is low (Fig. 9.2a). This will lead to smaller scale of flow separation thus better low end efficiency and better surge margin, although it will lead to choke capacity penalties. 
Advanced impeller

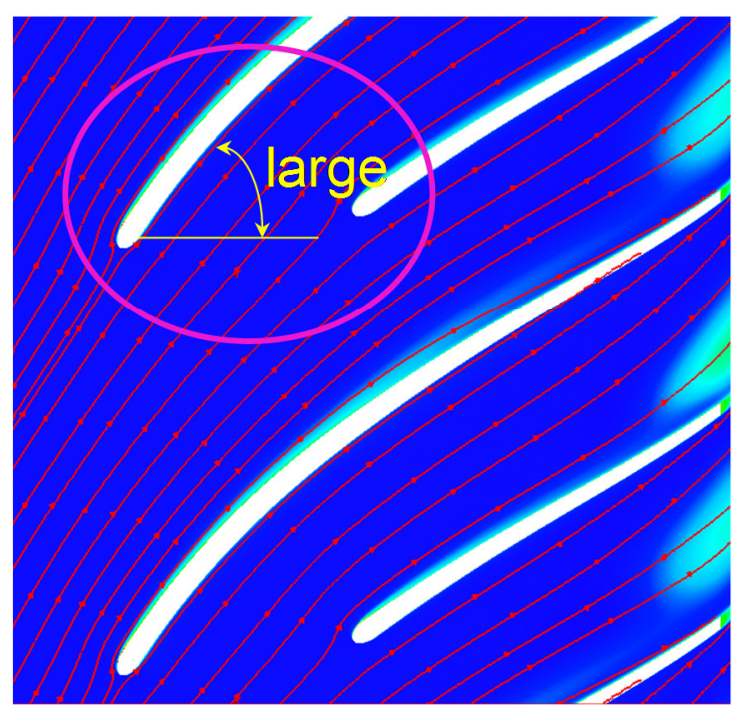

(a)

\section{Conventional impeller}

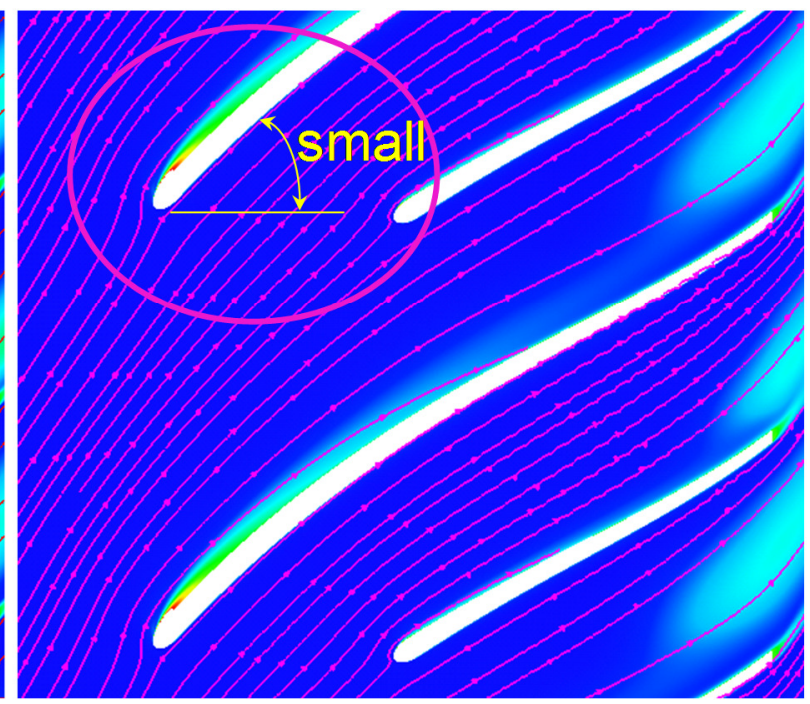

(b)

Fig. 9.2 Flow field CFD visualization inside a conventional and advanced LD compressor impeller

A numerical simulation tool, Numeca, was used by $\mathrm{Hu}$ [44] to validate the effectiveness of compressor performance of the advanced LD impeller design and active casing treatment. The simulation results indicated that the advanced compressor design for light duty diesel applications improved efficiency by almost $8 \%$ at low flow areas, compared to a production $\mathrm{IHI}$ light duty turbocharger that was used as donor turbo, while active casing treatment managed to keep the flow capacity at the same level as donor compressor (Fig. 9.3). Due to numerical converging issues in CFD simulations near surge conditions where the flow is physically unstable, the end of speed line at minimum mass flow rate in Fig. 9.3 does not represent the onset of surge.

A similar process for stress and structure analayses to the MD compressor development was also conducted to ensure the compliance of high cycle and low cycle fatigue requirement.

Figure 9.4 is the Campbell diagram of the advanced LD compressor that shows that at 145,000 design speed, the first blade flapping mode has 13\% margin above the 4 times of compressor excitation frequency. Again, 2618-T6 Al material is assumed.

Another key aspect of impeller design criterion is that the blade tip displacement has to be consistent with tip clearance. The CAE analysis, as illustrated by Fig. 9.5, shows that the maximum resultant displacement is $0.17 \mathrm{~mm}$, which is consistent with the blade tip clearance we use for this design.

Figure 9.6 shows the von Mises stress distribution of the advanced LD compressor impeller at design speed. The peak stress is located in the bore area, which is $76 \%$ of the material yield strength. Typically $75 \%$ of the yield strength is used as the guideline in the industry. The 1\% gap is easy to be addressed via either back face profile optimization as we did in MD compressor design or using boreless design in production. 


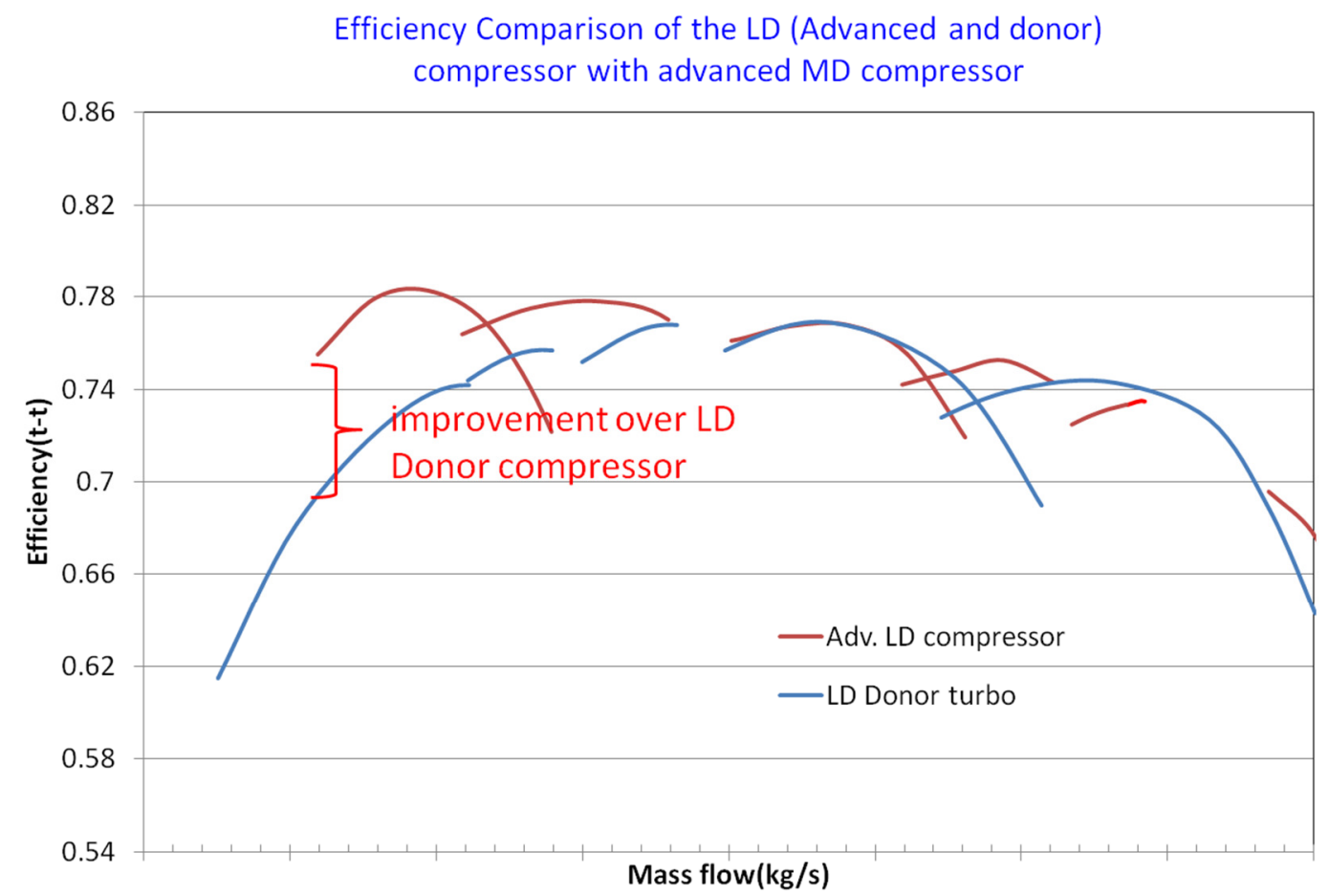

Fig. 9.3 Performance comparison of a conventional compressor (flow bench data) and advanced compressor with ACT (CFD analysis [14])

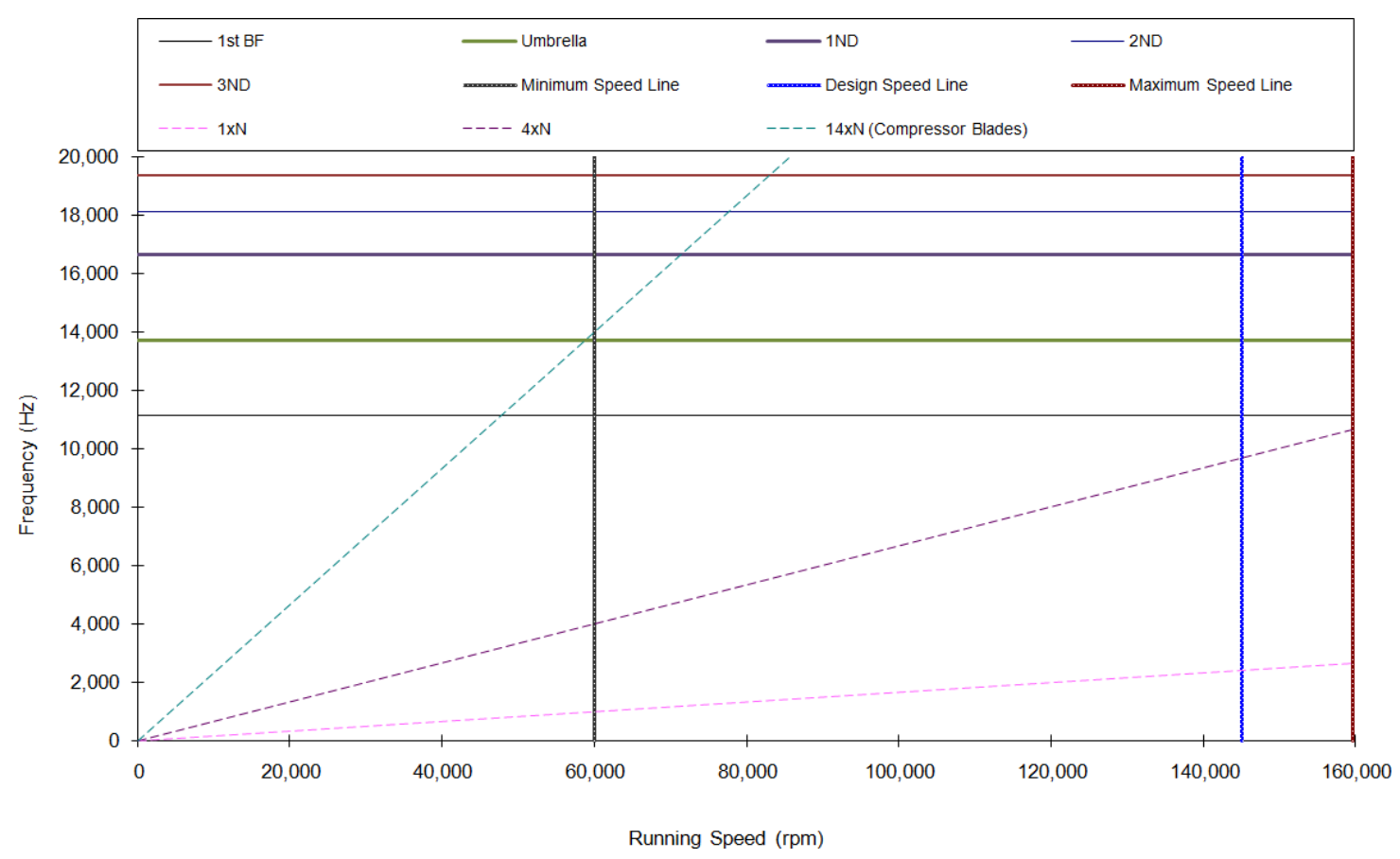

Fig. 9.4 Campbell diagram of advanced LD compressor impeller 

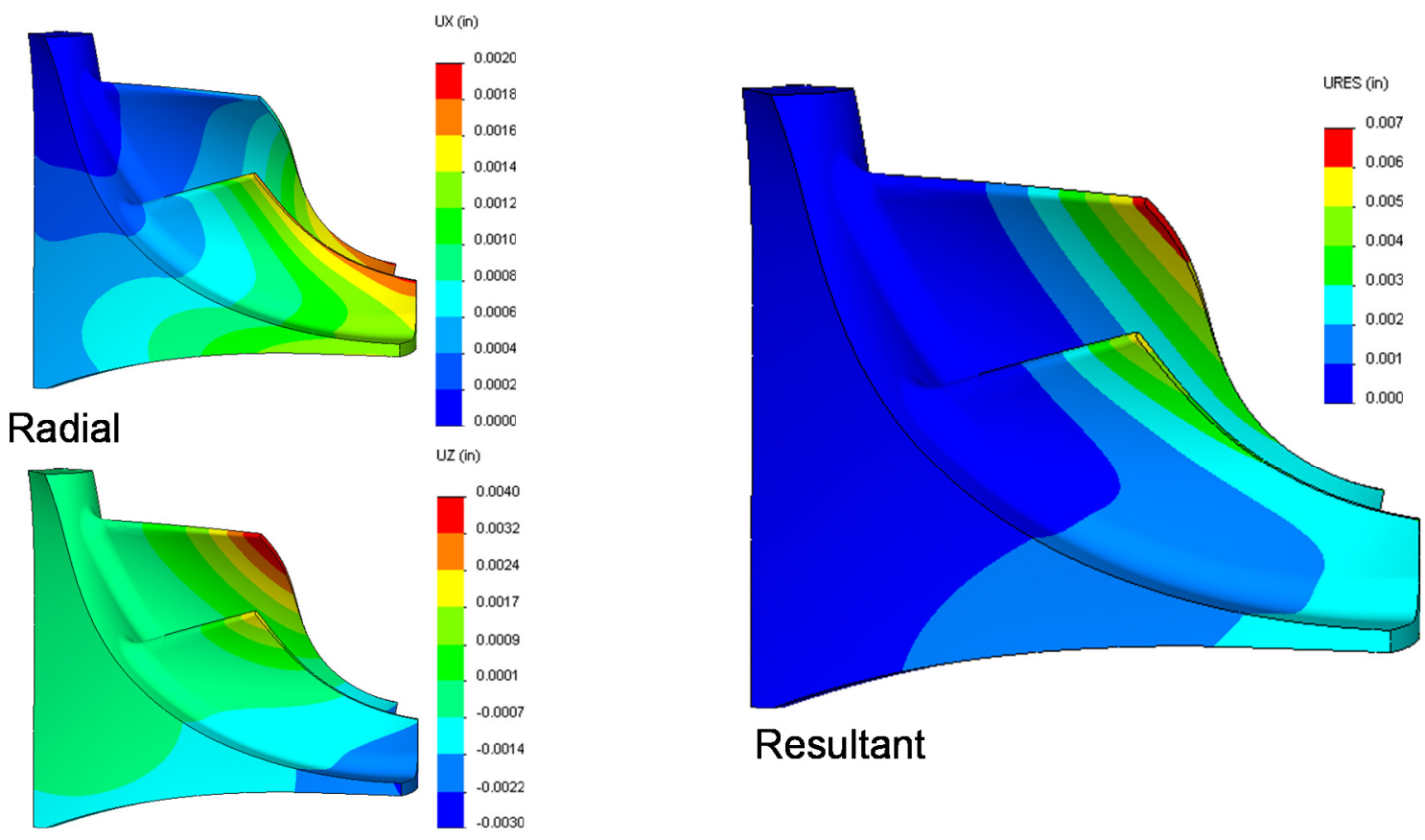

Resultant

\section{Axial}

Fig. 9.5 Advanced LD impeller displacement at rated speed (145,000rpm)
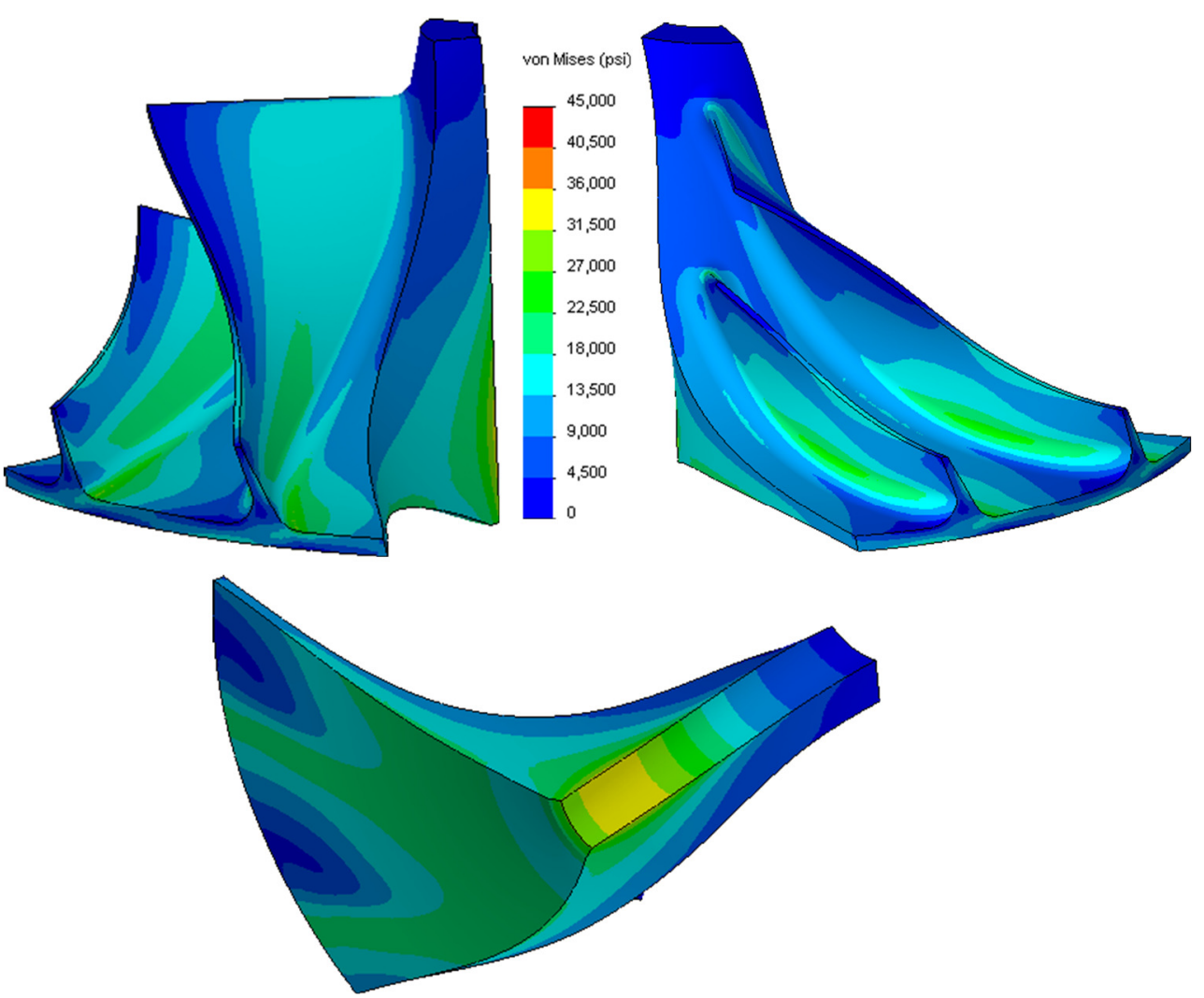

Fig. 9.6 Advanced LD impeller von Mises stress distribution at design 
9.3

Development of advanced LD mixed flow turbine

Conventional radial flow turbine designs have peak efficiencies at $\mathrm{U} / \mathrm{C}$ ratios between $0.6-0.7[4,51]$. Mixed flow turbines have long been recognized [46, 47] to have the capability to shift high efficiency zones to low U/C areas, even though the peak efficiency area may not necessarily be higher than a radial flow turbine. The intention at the time was focused on better utilization of pulsation energy out of engine exhaust, i.e. the instantaneous high pressure pulses push the operation point of the turbine into low $\mathrm{U} / \mathrm{C}$ areas where mixed flow turbines have been demonstrated that they can outperform conventional radial flow turbine designs. The benefit of mixed flow turbine with pulsation flow has been recognized in academia as most turbocharger performance is quantified by testing a turbocharger assembly on a steady state flow bench, and being limited to very small areas defined by stable operation of the compressor. When Ford started this advanced turbocharger research in 2006, the mixed flow turbine was selected as a key technology due to a totally different reason, the increased EGR requirement for future emission compliance driving operation at high expansion ratios and low $\mathrm{U} / \mathrm{C}$ areas for extended of time.

The mixed flow turbine is characterized by the non-radial inlet that reduces the flow path curvature and effectively reduces the secondary flow losses [47]. The other noticeable feature is that the radial flow turbine has the inducer blade angle parallel to the rotational axis vs. mixed flow turbines that typically have inducer blade angles between 2050 deg. relative to rotational axis. The inducer blade angle helps the inducer to adapt to different instantaneous incidence angles of incoming exhaust flow varying with the flow rate and, more importantly, the pulsed pressure [47].

At VGT full open positions, as the numerical analyses in indicated (Fig. 9.7), the advanced LD turbine, represented by "CN11-2" has substantially higher efficiency than the donor IHI turbine, compared on "w/o bearing loss" bases since the numerical prediction of turbine performance does not include the bearing loss, even though they have quite similar flow capacity at full open position (the full flow capacity is typically dictated by the VGT flow capacity, which is the same for both turbines). That means engine may deliver much higher power since the turbine inlet temperature will be lower due to the higher efficiency on the advanced LD mixed flow turbine, and lower pumping loss.

The numerical simulation also indicated that the mixed flow turbine ("CN 11-2" in Fig. 9.8) can dramatically improve the turbine efficiency in low $U / C$ areas when the turbine nozzle vane is fully closed (e.g. engine idle condition). It has to be noted that due to pulsation flow out of engine, the operation point of a turbine may momentarily push much lower than 0.4 of $\mathrm{U} / \mathrm{C}$ at the peak of exhaust pressure, i.e. beyond the point that can be typically characterized by a steady state flow bench. In other words, the mixed flow turbine may potentially extract more exhaust energy, which unfortunately may not be quantified from steady state turbine maps and have to be validated on an engine dynamometer test.

Compared with the advanced MD and LD mixed flow turbine wheel, Fig. 9.9, instead of 13 blades, the LD mixed flow turbine has 11 blades since the donor turbo has 15 thin nozzle vanes. Also the larger inducer diameter, matched to a relatively large compressor size for the purpose of better low end efficiency, drives to a narrow inducer blade, which may compromise the performance of a mixed flow turbine at low $\mathrm{U} / \mathrm{C}$ area. Initial LD mixed flow turbine had scallop cut on the back face, similar to the donor turbine. The scallop cut was eliminated after blade and back face optimization, due to turbine efficiency penalty concerns. 


\section{Turbine efficiency at full open position}

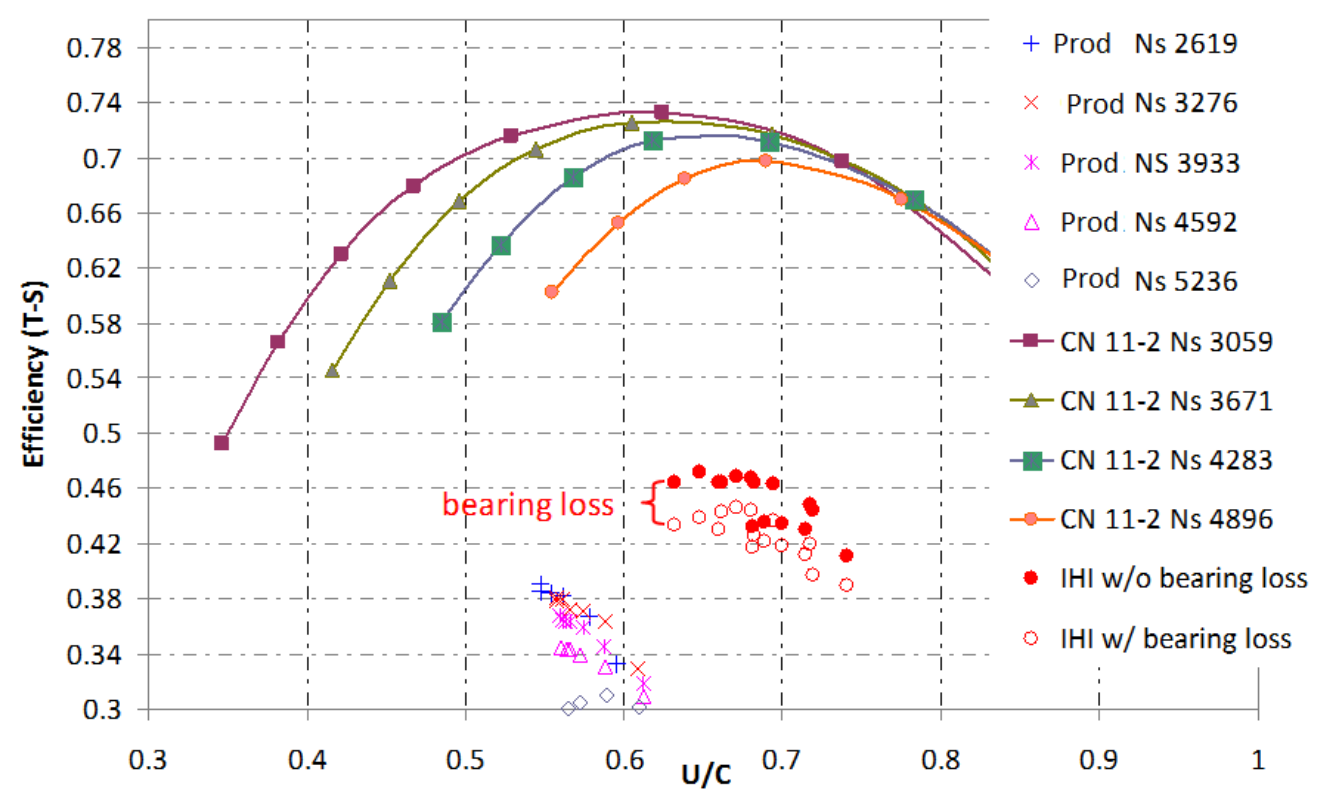

Fig. 9.7 Comparison of turbine efficiency between conventional and adv. LD mixed flow turbines at full open positions

Turbine efficiency at full close position

CN11-2 (90deg) vs. GT22

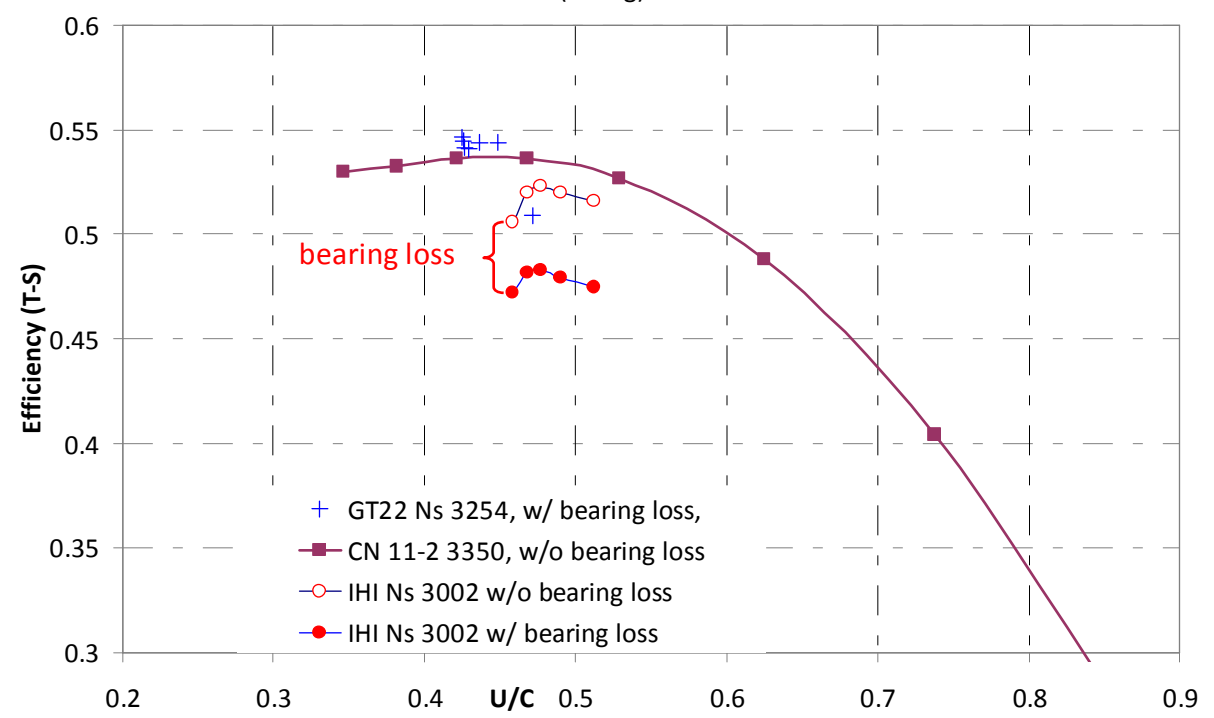

Fig. 9.8 Comparison of turbine efficiency between conventional and adv. LD mixed flow turbines at full close positions 

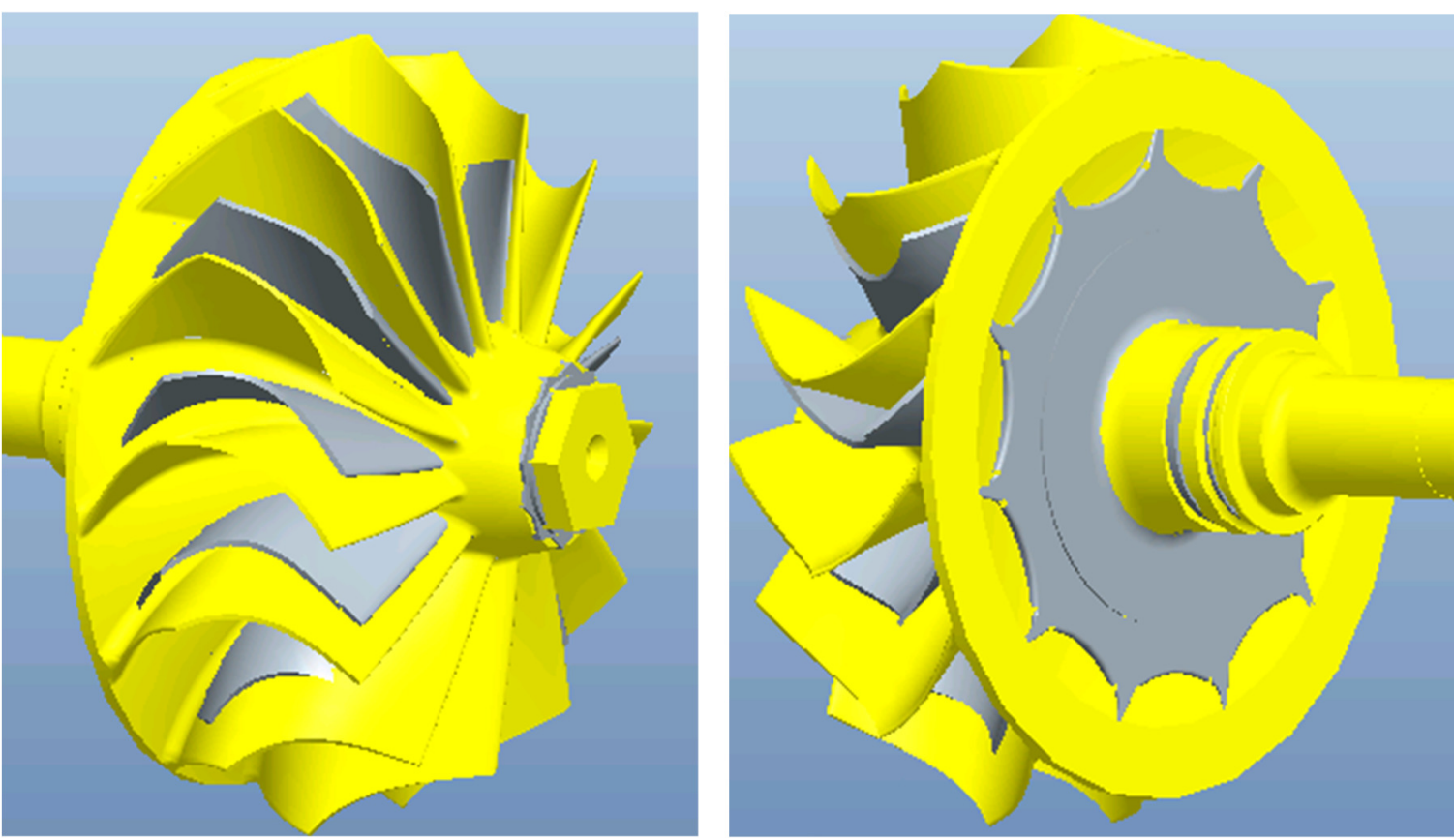

Fig. 9.9 Comparison of advanced MD (grey) and LD (yellow) mixed flow turbine

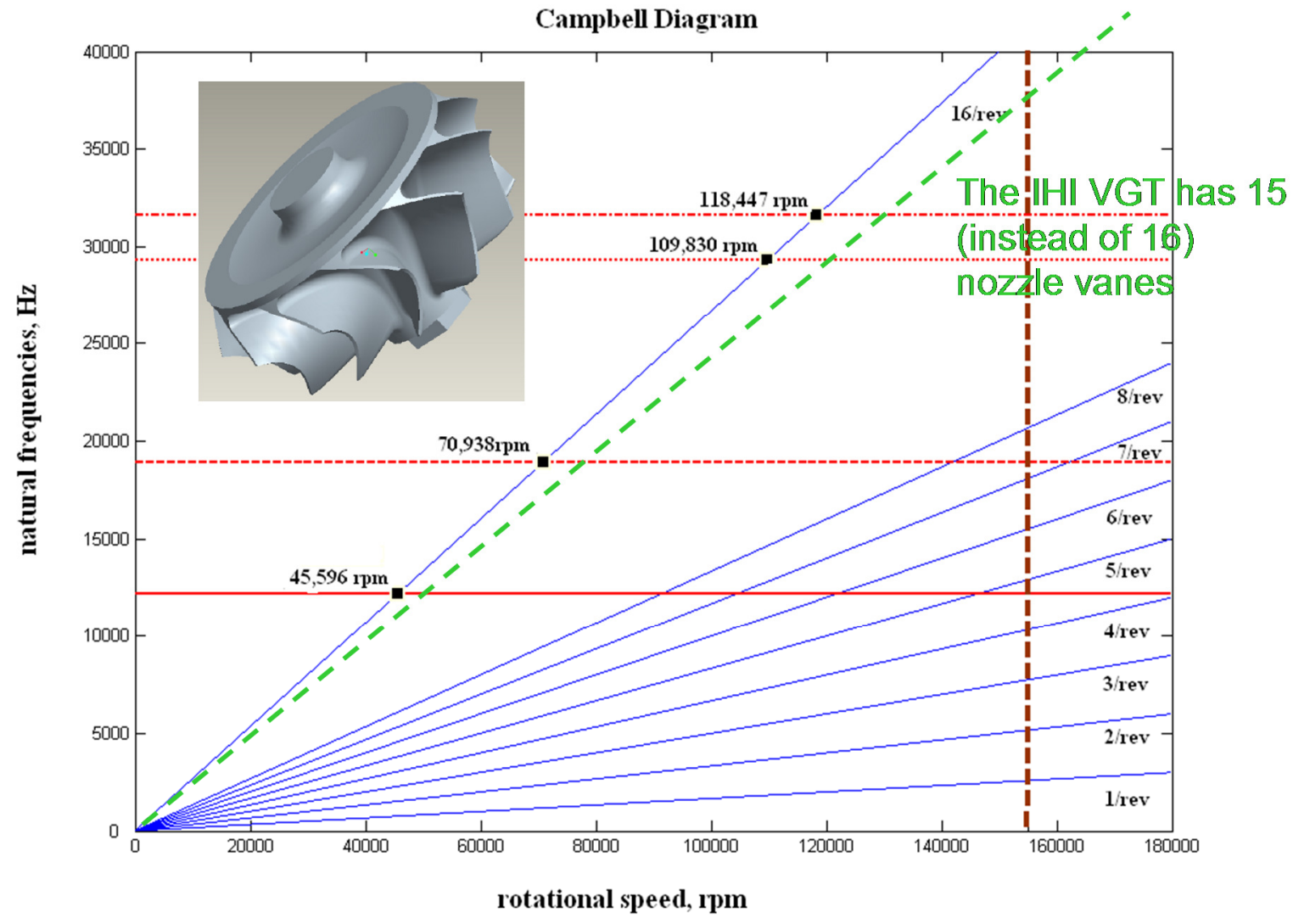

Fig. 9.10 Campbell diagram of advanced LD mixed flow turbine 
The modal analysis, as shown by the Campbell diagram in Figure 9.10, shows that the first order natural frequency is $12,159 \mathrm{~Hz}$ and the natural frequency to operation frequency is 4.9 , sufficient even for commerical applications.

10 Stead state flow bench performance validation of advanced LD turbocharger

The aforementioned advanced LD turbocharger, with ruled surface compressor impeller, active casing treatment and mixed flow turbine, was fabricated and flow bench tested at the turbocharger manufacturer facility for better consistency in turbocharger measurement as it is well understood that steady state flow bench tests, especially the turbine efficiency measurement, are subject to certain level uncertainty.

Due to time constraints and other reasons, fabrication quality of the prototype advanced LD turbocharger is very disappointing. The fabrication quality issues resulted in poor tip clearance control. The smaller than desired compressor tip clearance limited the test speed to135,000rpm.

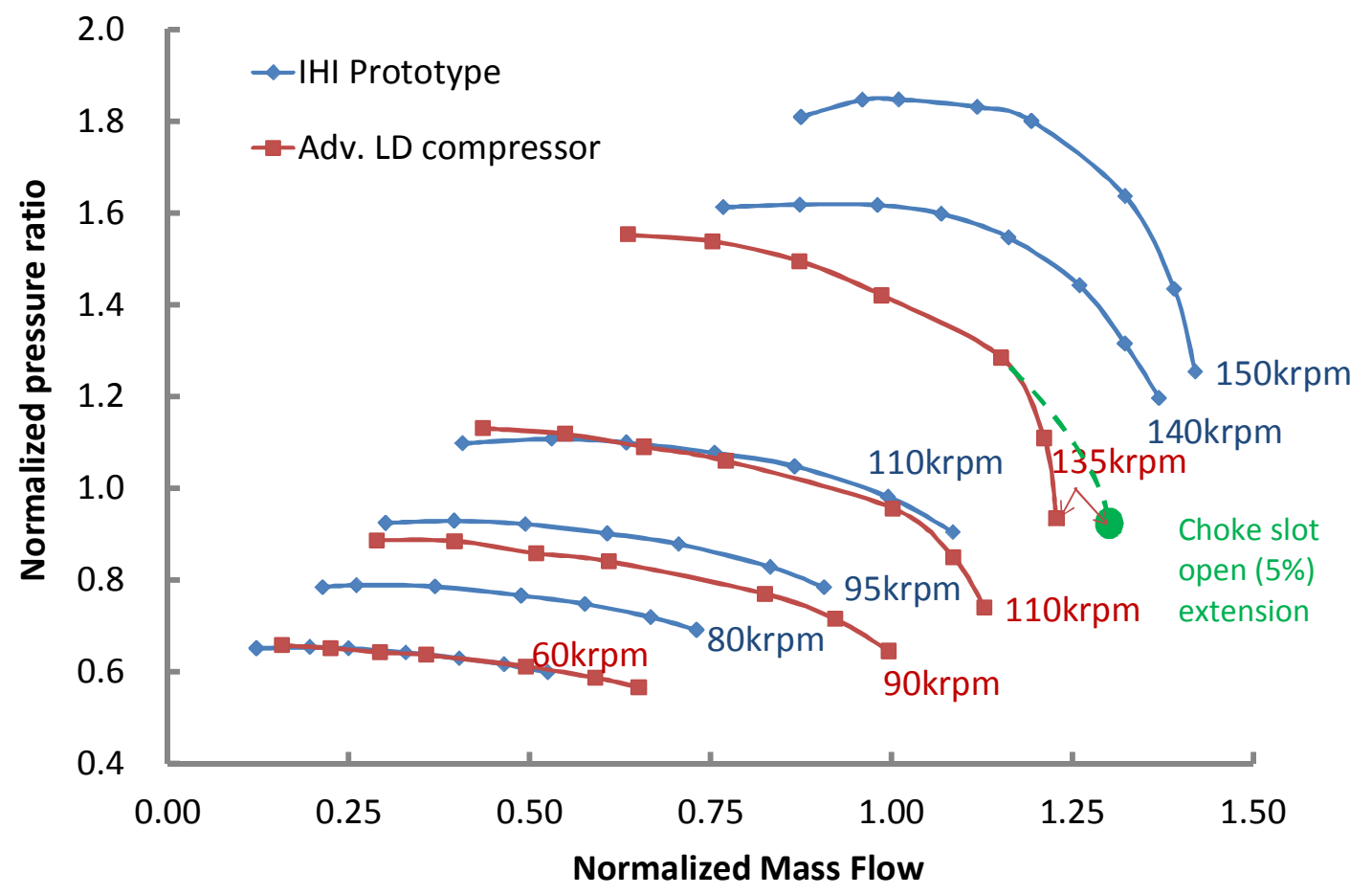

Fig. 10.1 Steady state flow bench test of pressure ratio (t-t): base vs. advanced compressor

The flow bench test data (Fig. 10.1 and Fig. 10.2) show that the advanced compressor impeller, despite the rule surface, delivers improved surge margin by $\sim 21 \%$, and narrower stall area (represented by flat or positive pressure ratio slope on speed lines.) Again, the surge or stall margin improvement is mainly limited to high pressure ratio 
areas. At 135,000rpm, with the choke slot open, the active casing treatment demonstrated $5 \%$ flow capacity improvement, which is less than what is demonstrated on the advanced MD compressor. It is anticipated when the impeller is in a "deep choke", the choke slot in the active casing treatment will deliever much more flow capacity extension.

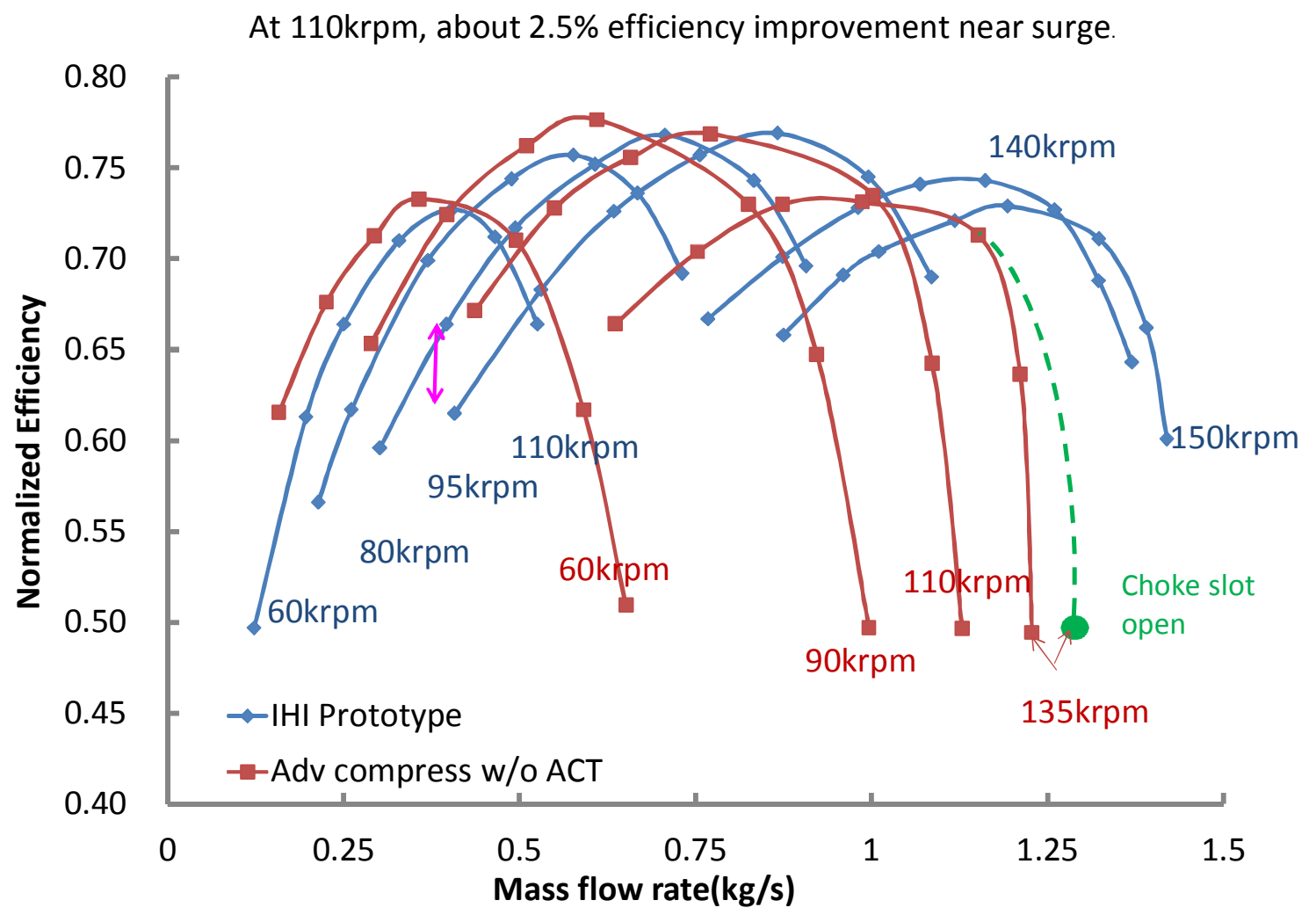

Fig. 10.2 Steady state flow bench test of efficiency (t-t): base vs. advanced compressor

The advanced LD compressor seems to improve the efficiency at $2.5 \%$ at $110,000 \mathrm{rpm}$ and, more importantly, around $8 \%$ at $60,000 \mathrm{rpm}$ turbo speed. The turbo speed 60,000rpm is almost right in the center of customer driving cycle, which is critical for vehicle fuel economy improvement. The efficiency improvement at low end (Point " $D$ " in Table 9.1) of the advanced LD compressor is lower than desired target. Based on this incomplete data, there is reasonable confidence that the advanced LD compressor can achieve the design target listed in Table 9.1, should the fabrication quality allows the prototype LD turbo to be operated at rated speed, 145,000rpm, with the active casing treatment.

This flow bench test data of compressor efficiency shows that the typical engineering trade-off: while saving the manufacturing cost, the ruled surface cannot deliver the same level of efficiency and flow range as that of a compressor impeller with arbitrary surface, even with the active casing treatment. 


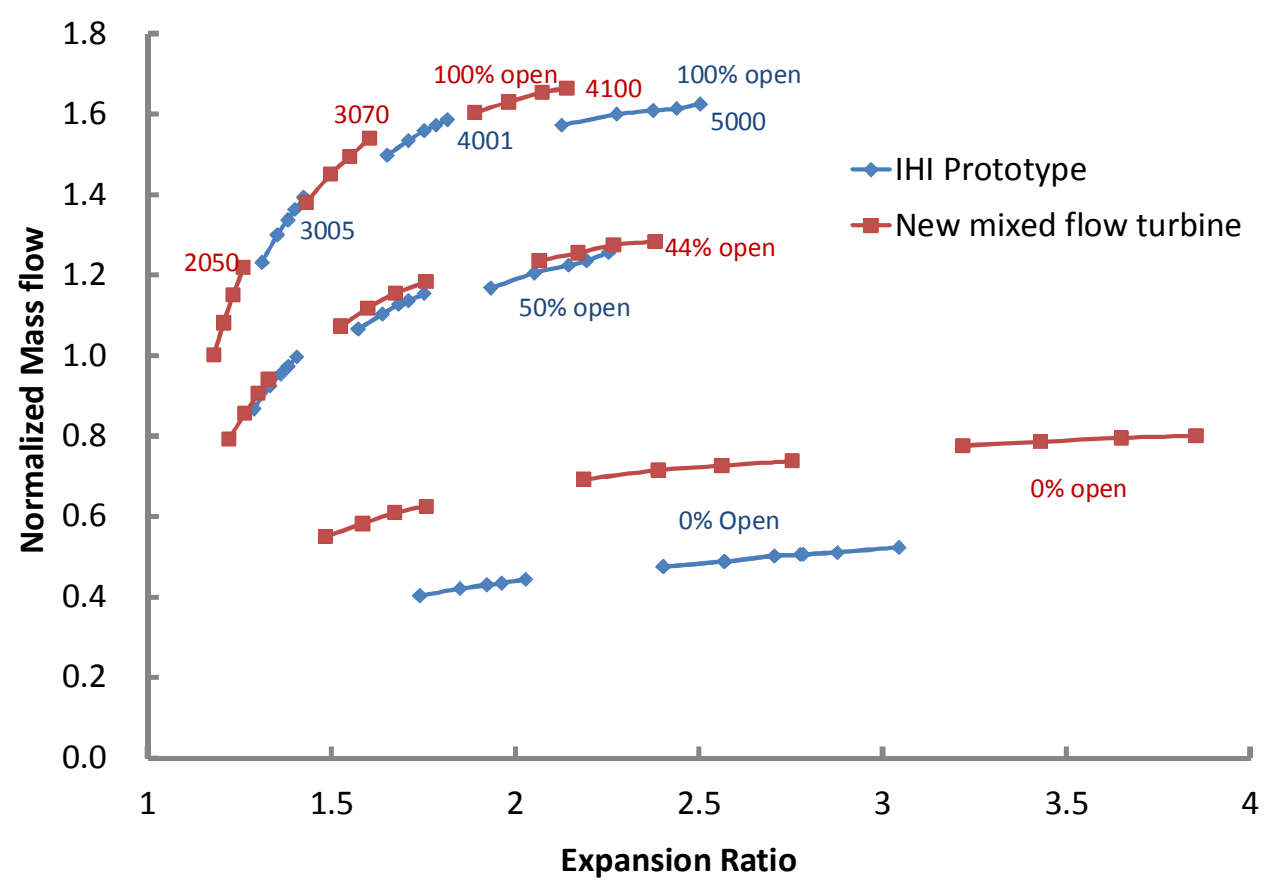

Fig. 10.3 Steady state flow bench test reduced mass flow (t-s): base radial vs. advanced mixed flow turbine

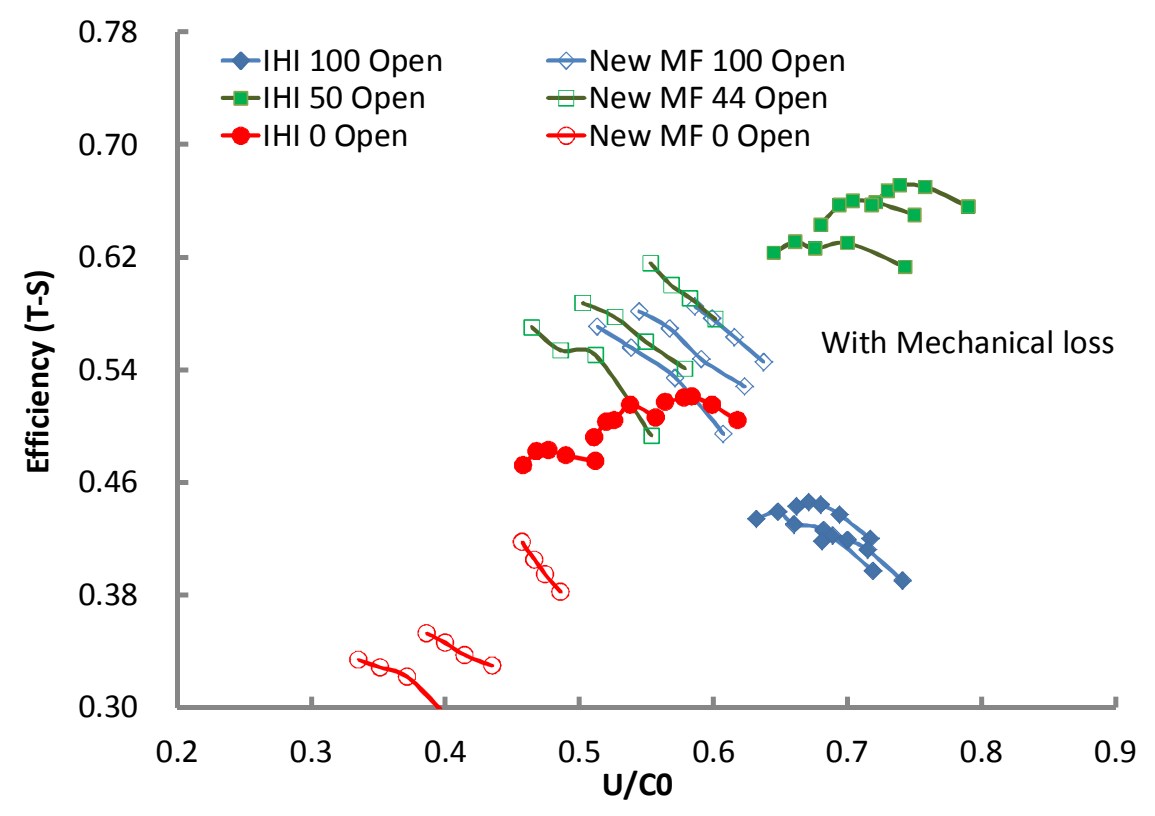

Fig. 10.4 Steady state flow bench test of efficiency (t-s): base radial vs. advanced mixed flow turbine 
The design of compressor with ruled surface impeller even affects the optimal design of mixed flow turbine that the compressor has to match with. Since the efforts were made to improve the surge margin and low end efficiency on the compressor, large blade angle at the leading edge of compressor impeller was used. A large diameter compressor wheel, relative to its flow capacity, was used. As a result, a relatively large mixed flow turbine has to be designed, i.e. the narrower inducer blade height was used to maintain the same inducer flow area, which may have limited the efficiency enhancement, especially at small vane open positions.

Figure 10.3 shows the comparison of reduced mass flow vs turbine expansion ratio between the base $\mathrm{IHI}$ radial flow turbine and the new advanced mixed flow turbine. The maximum flow capacity at full open position are identical since the maximum flow capacity is typically dictated by the VGT mechnisms that are identical; The efficiencies of the advanced mixed flow turbine (Fig. 10.4) have significant improvement at full open positions over the base $\mathrm{IHI}$ radial flow turbine, especially at lower $\mathrm{U} / \mathrm{C}$ area, i.e. when the turbine operates at high turbine inlet temperature, high expansion ratio such as choke conditions, the mixed flow turbine would show much higher useable flow capacity or higher turbine inlet temperature constrained rated power on engine. This conclusion is consistent with the analyses discussed in section 9.3 (Fig. 9.7).

The flow capacity at partial vane open positions, 50\% open for base IHI turbine and $44 \%$ open for advanced mixed flow turbine (the discrepancy is due to the VGT actuation calibration difference), are almost identical (Fig. 10.3). The efficiencies of advanced mixed flow turbine are lower at $\mathrm{U} / \mathrm{C}$ around 0.7 where radial flow typically outperform mixed flow turbine while at the U/C below 0.5 , the advanced mixed flow turbine outperforms the base IHI radial flow turbine (Fig. 10.4), which is anticipated and benefitial since future diesel engines, with high EGR application, would spend majority of duty cycle in partial VGT open position with U/C between 0.3-0.6.

The flow bench tested performances at VGT full close positions are a little hard to draw a solid conclusion from the steady state flow bench test alone. One main reason is that they were physically operated at different VGT open positions even though they were commanded to be "full close" (Fig. 10.3). Typically smaller vane position near full close position would deliver inferior performance. But is impossible to quantify this correlation. Again, the base IHI radial flow turbine shows better efficiencies at higher U/C (Fig. 10.4). But how the efficiency deteriorates towards low U/C (e.g. U/C below 0.4 ) area cannot be reliably predicted since the turbine cannot be characterized at low $\mathrm{U} / \mathrm{C}$ area. The only feasible approach to evaluate the turbine performance in the low $\mathrm{U} / \mathrm{C}$ area is to conduct engine dynamometer test where the exhaust pulsation flow may momentarily drive the $\mathrm{U} / \mathrm{C}$ to very low area, not being constrained by the energy balance between turbine and compressor. system.

All the efficiencies in Figure 10.4 included the mechanical loss of the bearing

The ultimate approach to evaluate the difference between conventional and advanced turbochargers, particularly with changes in turbine design, is to conduct engine dynamometer validation testing. Figure 11.1 is the experimental setup of the engine dynamometer test, which includes a production light duty diesel (3.2L) engine with a high pressure EGR system and a full aftertreatment system. Efforts have been made to 
calibrate this base engine with the base IHI turbocharger for Tier II Bin 5 emission level with DOC, DPF and urea SCR catalysts. The advanced turbocharger developed in this project includes the aforementioned advanced compressor impeller with ruled surface, active casing treatment (ACT) and advance mixed flow turbine with the VGT mechanism and center housing identical to base $\mathrm{IHI}$ turbocharger.

Due to the constraints of test cell time, steady state engine mapping was mainly limited to the area that is large enough to cover FTP cycle, i.e. the highest BMEP points in Fig. 11.2 do not represent the full load of this engine. For the same reason, the base engine (including VGT, EGR, AFR, etc.) and the aftertreatment calibration, especially the cold engine calibration was by no means optimal for these two turbochargers. It was simply one calibration that met Tier II Bin 5 emission standard and used for both base (IHI) turbo and advanced (TS11) turbo.

Figure 11.2 shows the steady state part load engine test results with two different turbochargers, ranging from 1250 to 2000rpm that covers majority of the FTP cycle. The advanced compressor had a nearly $10 \%$ compressor efficiency improvement over that of the base compressor, consistent with the 2 - 4\% BSFC improvement in the mapped areas except a few places where turbine performance and VGT or EGR calibration dominated the engine performance. The low BEMP area at 2000rpm was one of the exceptions; this operation point had a relatively high turbo speed, low expansion ratio across the turbine wheel and a relatively low turbine inlet temperature that pushed turbine operation into high $\mathrm{U} / \mathrm{C}$ areas where mixed flow turbines have efficiency that may be inferior to radial flow turbine. Therefore, the advanced mixed flow turbine may not be as competitive as conventional radial flow turbine in some operation conditions or applications.

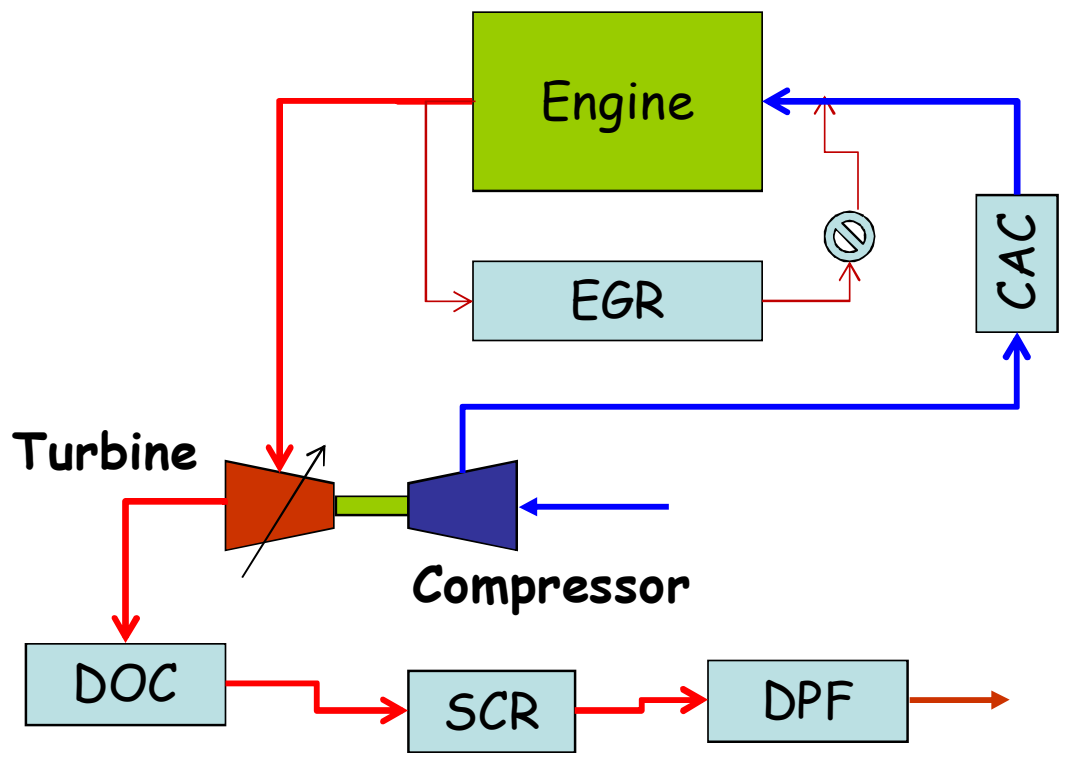

Fig. 11.1 Setup of engine dynamometer experiment will full aftertreatment (DOC+DPF and urea SCR) 
Compressor Efficiency and BSFC at 1250rpm

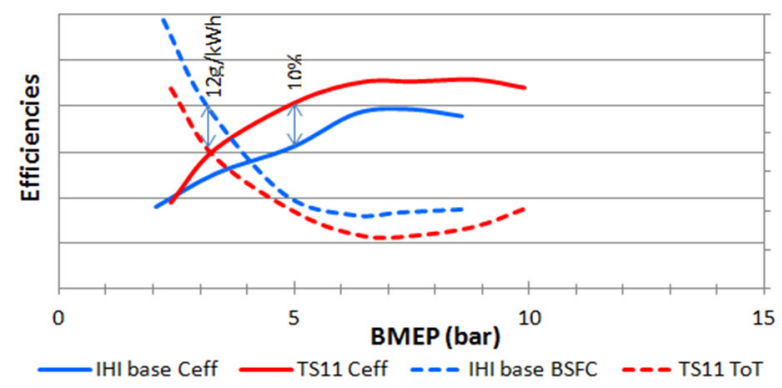

Compressor Efficiency and BSFC at 1750rpm

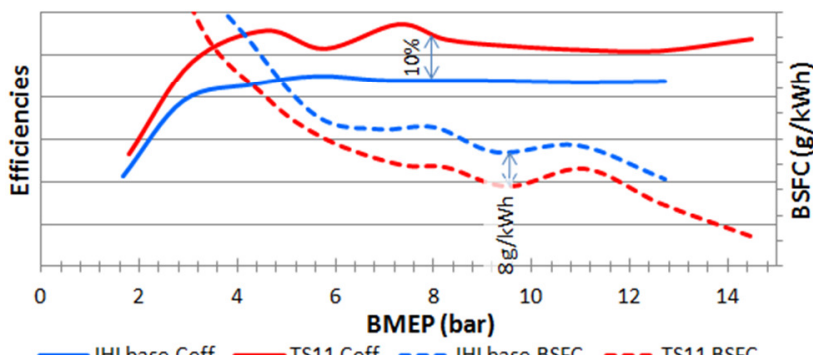

Compressor Efficiency and BSFC at 1500rpm

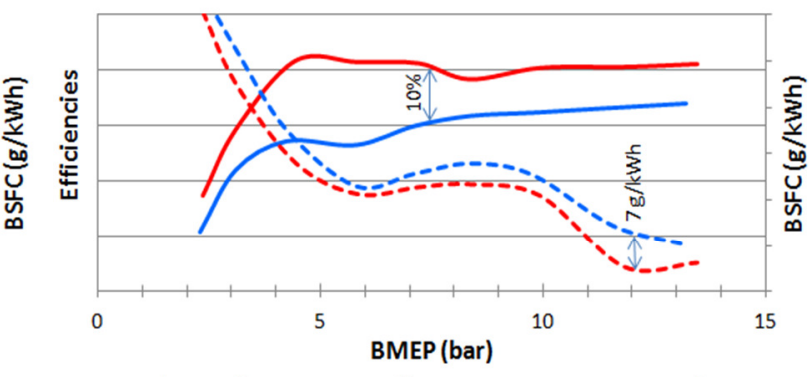

—IHI base Ceff —TS11 Ceff --- TS11 BSFC --- IHI base BSFC

Compressor Efficiency and BSFC at 2000rpm

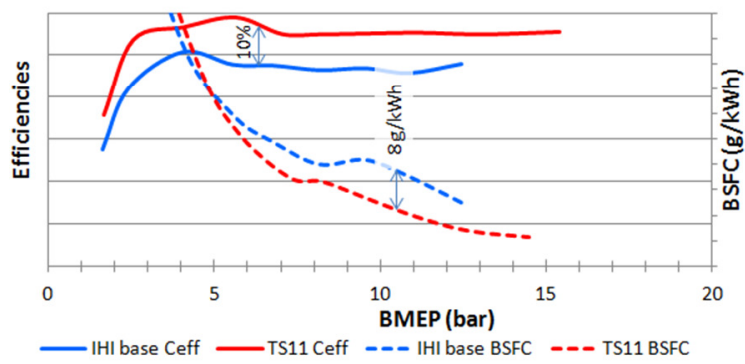

Fig. 11.2 Comparison of steady state part load engine performance with different turbochargers

To quantify the impact of the advanced turbo technology on engine performance, for better thermal efficiency or aftertreatment conversion efficiency, small sets of operating points on a warm engine are not sufficient. Engines are increasingly becoming highly integrated, dynamic systems that contain many highly interactive subsystems and components that can only be evaluated under fully transient conditions with a fully integrated system. Due to that consideration, extra efforts were made to calibrate the engine for transient FTP operation with a full aftertreatment system, including DOC, urea SCR and DPF to evaluate the turbocharger benefit over a complete cold transient FTP cycle with Tier II B5 tailpipe emissions.

The engine was first run with the base IHIF55 turbocharger. The base engine came with Tier2 Bin 8 emission. There is no additional combustion development made except that the steady state and transient calibration was optimized to achieve Tier2 Bin5 emission on a cold FTP cycle as below:

\begin{tabular}{|c|c|c|c|c|c|c|c|c|}
\hline Bag & HC & $\mathrm{CO}$ & NOx & Energy & fuel & $\begin{array}{l}\text { Thermal } \\
\text { efficiency }\end{array}$ & \multicolumn{2}{|c|}{$\begin{array}{l}\text { AT conversion } \\
\text { efficiency (\%) }\end{array}$} \\
\hline number & gr/mile & gr/mile & gr/mile & $k J$ & kg & $(\%)$ & $\mathrm{HC}$ & NOx \\
\hline 1 & 0.040 & 0.175 & 0.053 & 7570 & 0.622 & $28.4 \%$ & $86.4 \%$ & $69.6 \%$ \\
\hline 2 & 0.038 & 0.017 & 0.012 & 7476 & 0.615 & $28.4 \%$ & $94.4 \%$ & $97.2 \%$ \\
\hline 3 & 0.008 & 0.006 & 0.005 & 7556 & 0.550 & $32.1 \%$ & $95.9 \%$ & $97.6 \%$ \\
\hline Total & 0.087 & 0.199 & 0.071 & 22602 & 1.787 & & $91.5 \%$ & $88.2 \%$ \\
\hline \multicolumn{5}{|c|}{ Cycle thermal efficiency \% } & $29.6 \%$ & & & \\
\hline
\end{tabular}


aAfter having the base Puma 15 engine with base IHI turbo successfully pass the T2B5 tailpipe emissions with full aftertreatment, the turbocharger was switched to the advanced LD turbocharger. The identical calibration and aftertreatment was used for the FTP transient test as well as the steady state part load tests.

Table 11.1 summarizes the results of cold transient FTP testing with two different turbochargers and the same calibration meeting Tier II Bin 5 tailpipe emissions. The average thermal efficiency when running with the advanced turbocharger (TS11) over the entire FTP cycle shows more than $3 \%$ improvement vs. the conventional production $(\mathrm{IHI})$ turbocharger.

It is noteworthy to mention that the $3.3 \%$ thermal efficiency improvement from this advanced turbocharger may be a conservative estimate since the test performed did not include other potential benefits. For instance, due to superior performance at medium to high vane open positions, the advanced turbo design can run at higher boost, producing higher torque at low engine speed than the conventional turbo, allowing the transmission shift schedule to be optimized so that the engine can operate more time at low engine speeds and high torque area to save additional fuel.

\begin{tabular}{|r|r|}
\hline & $\begin{array}{r}\text { FTP cycle } \\
\text { thermal efficiency }\end{array}$ \\
\hline IHI Base Turbo & $29.55 \%$ \\
TS11 Adv. Turbo & $30.52 \%$ \\
Improvement & $3.3 \%$ \\
\hline
\end{tabular}

Table 11.1. Summary of cold FTP transient cycle engine dynamometer tests

The development of MD compressor has the best trade-off between high end flow capacity and low end efficiency, as described in details in Chapter 4, having the manufacturing cost as secondary consideration, e.g. the compressor impeller has arbitrary surfaces. The development of LD compressor has more focus on cost. Therefore the compressor impeller uses ruled surface that drives up diameters of both compressor and turbine wheels.

As part of contract amendment with DOE, we decided to revisit the MD turbocharger design to shift more focus on manufacturing cost, i.e. design a version of an advanced MD compressor impeller with ruled surfaces that can be flank milled at much lower manufacturing cost, and a mixed flow turbine accordingly, for those cost sensitive applications. As a result, a production Honeywell GT37 turbocharger, with larger frame size, was selected as donor turbocharger for the MD2 development. Due to the time and budget constraints, the development of advanced MD2 turbocharger was limited to design, analyses, fabrication of prototype compressor and mixed flow turbine wheels. The turbocharger assembly and flow bench test was not included under this project. 
12.1 Design and analyses of advanced MD2 compressor

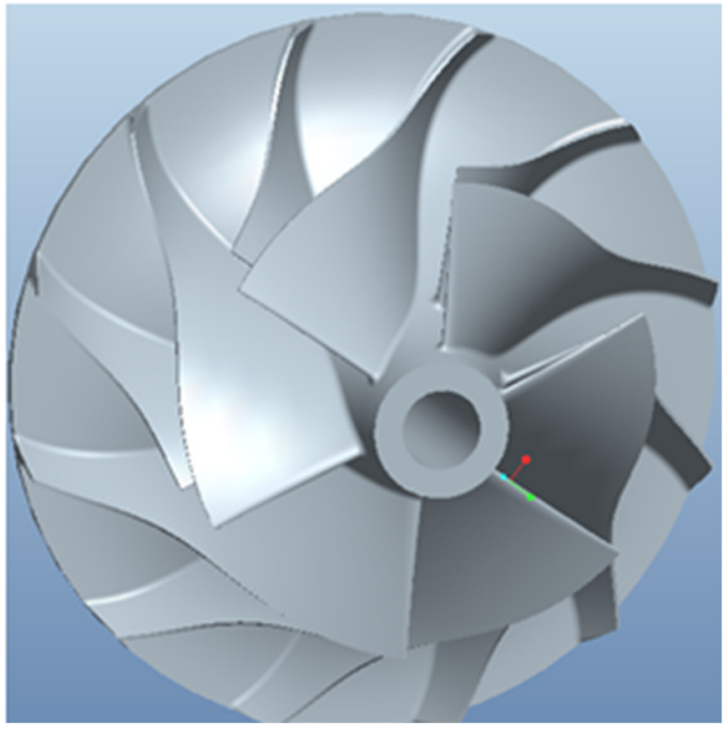

Fig. 12.1. Design of advanced MD2 compressor featured with ruled surface impeller

Figure 12.1 shows the design of the advanced MD2 compressor with ruled surface impeller that can be flank milled for lower manufacturing cost. It has 6 split blades and 6 full blades for better compressor efficiency and operation range trade-off, while the base GT37 has full blades only therefore the advanced MD2 compressor, with active casing treatment, can deliver sufficient flow capacity with $93 \%$ of the base compressor exducer diameter, which is favorable for stress, turbine sizing, rotor inertia thus transient response.

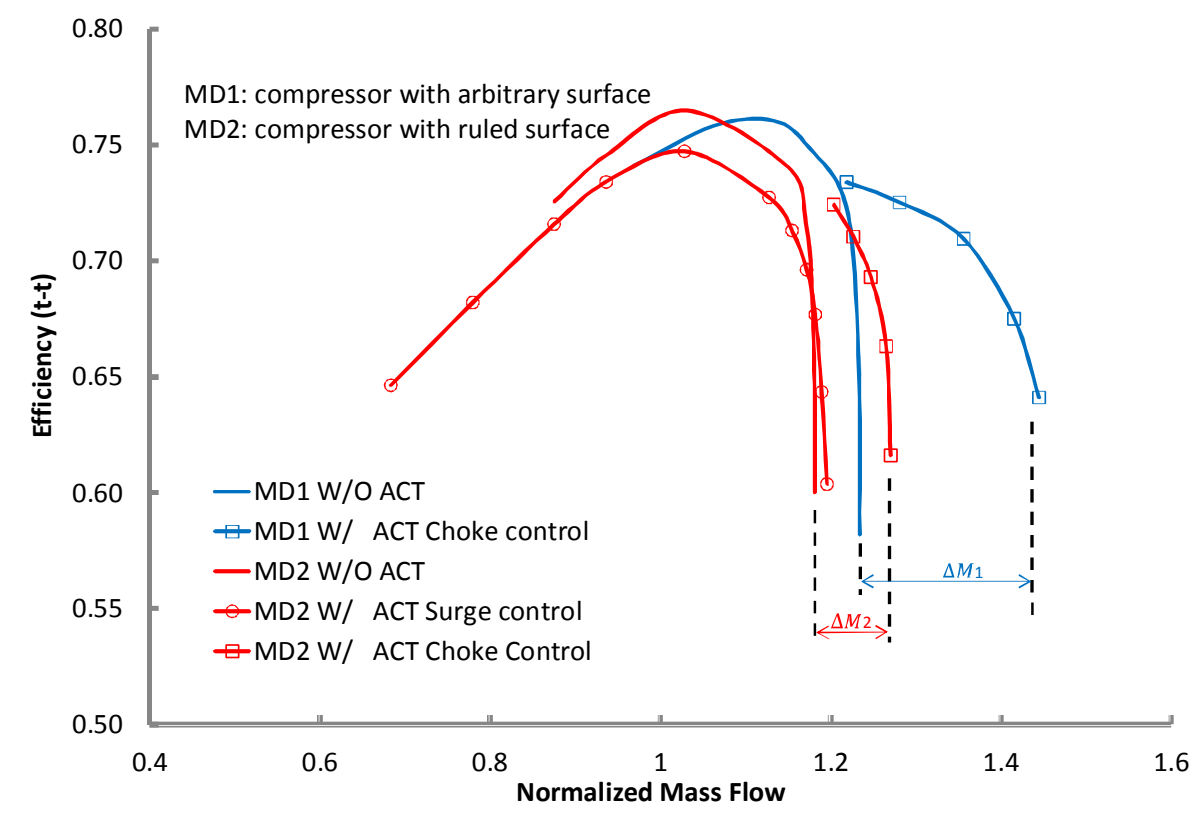

Fig. 12.2. CFD prediction of advanced MD2 compressor efficiency with and w/o active casing treatment at peak turbo speed 


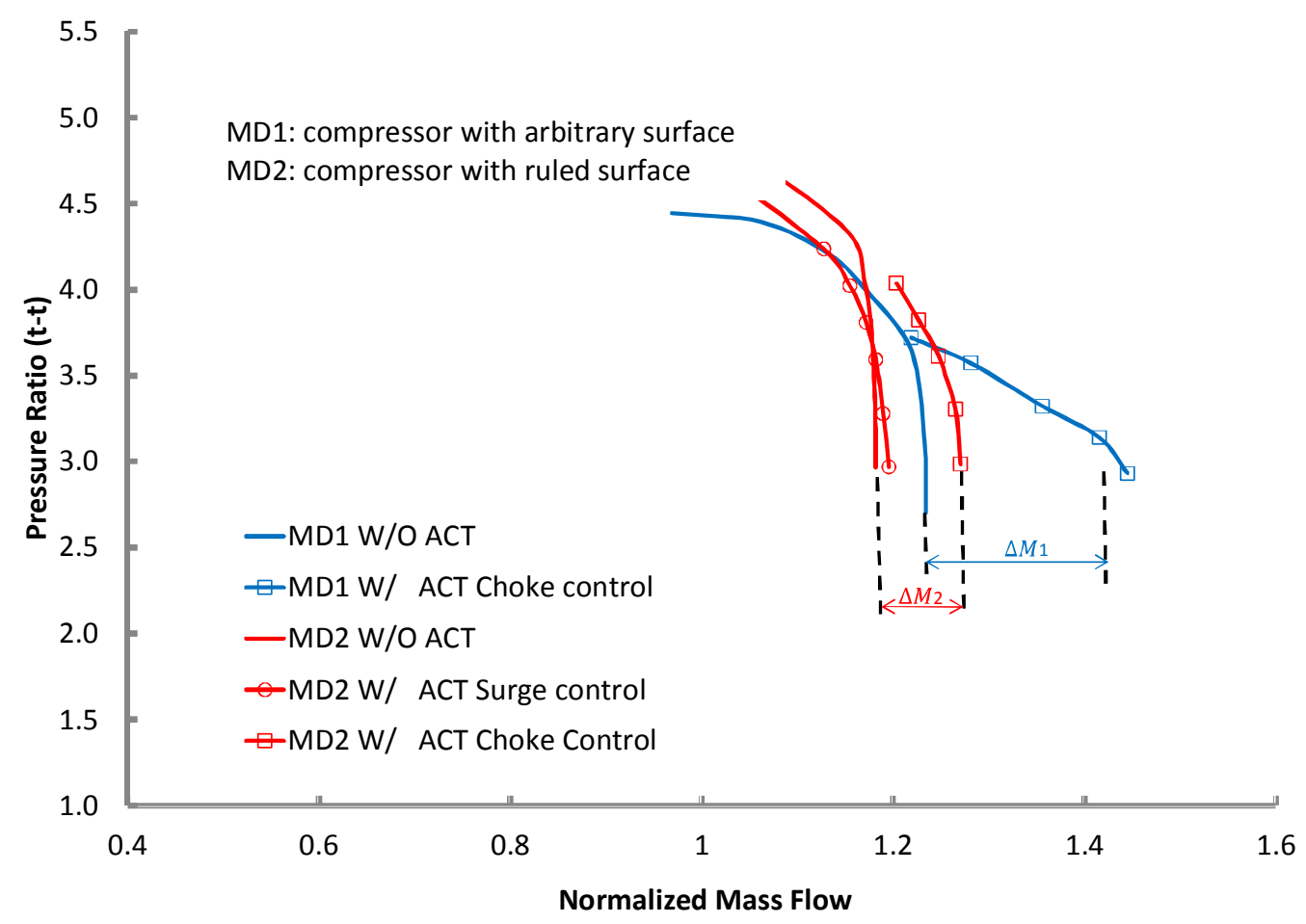

Fig. 12.3. CFD prediction of advanced MD2 compressor pressure ratio with and w/o active casing treatment at peak turbo speed

The 3D CFD prediction (Fig. 12.2 and 12.3) indicates that the advanced MD2 compressor design can achieve almost the similar performance as the advanced MD1 compressor that has an arbitrary surfaced impeller (if 3.5 pressure ratio is needed for rated operation condition), when active casing treatment is used, except that the impeller with ruled surface is $8 \%$ larger in diameter. The CFD based optimization helps to gain $8 \%$ of extra flow capacity with the active casing treatment for MD2 compressor wheel.

Similar to the advanced LD compressor design, the smaller wheel, especially with the large blade angle, the compressor efficiency at small mass flow area, along with surge margin, is expected to be improved.

The modal analysis shows that the first order natural frequncy is 4.4 times of max operation frequency of the MD2 compressor (Fig. 12.4). The stress analyses indicated (Fig. 12.5) that the peak stress is located in the fillet area on the compressor hub surface, which is $5 \%$ higher than design target and could be either over predicted due to coarse mesh or mitigated via larger fillet radius which has very little impact on the aerodynamic performance. The stress in the bore area, as shown in Fig. 12.6, is within design target since area is exposed to lower temperature where the material has higher strength. Certainly boreless design is always available to reduce the bore stress if it is necessary. 


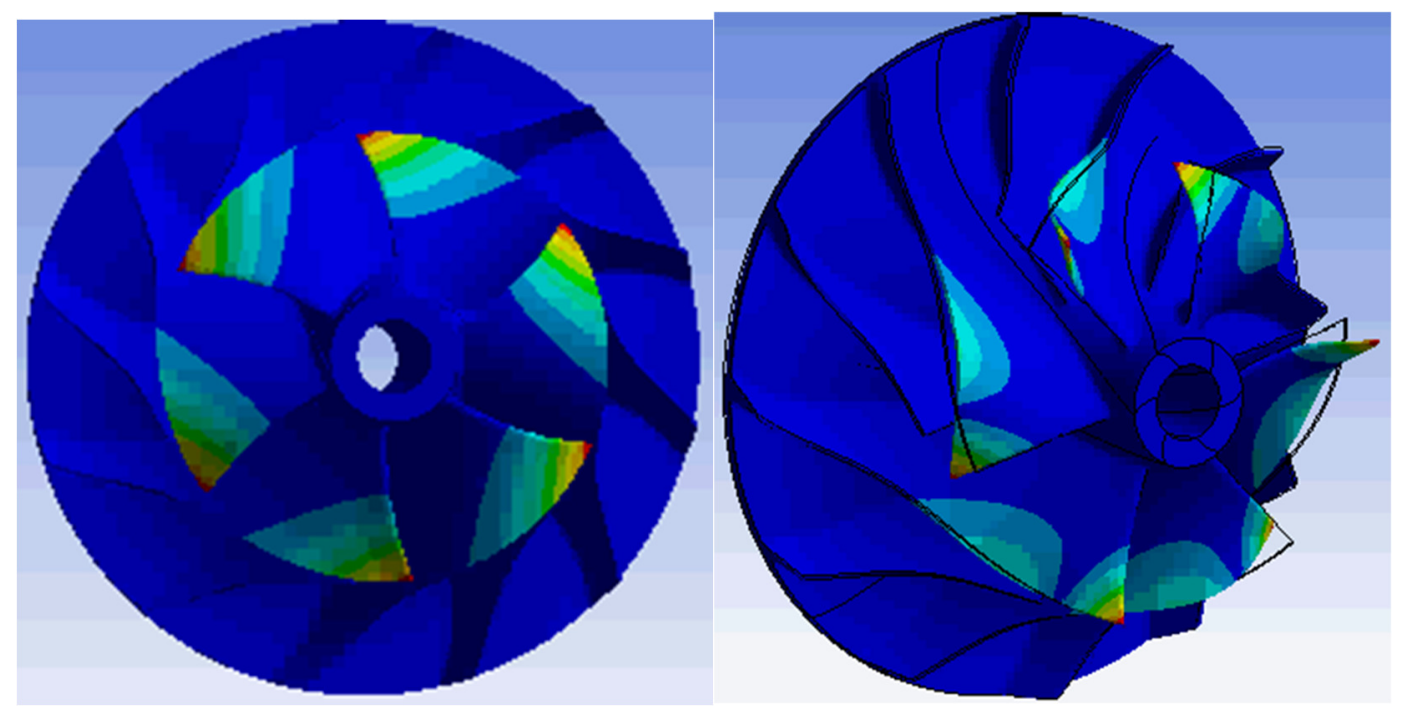

Fig. 12.4. Modal analysis of advanced MD2 compressor wheel: $1^{\text {st }}$ order (left) and $2^{\text {nd }}$ order (right)

\section{G: Axial5.2mmR70D9 \\ Equivalent Stress \\ Unit: MPa \\ Time: 1 \\ $7 / 9 / 20128.39 \mathrm{PM}$

\begin{tabular}{|l|l}
321.54 Max \\
285.82 \\
250.11 \\
214.39 \\
178.67 \\
142.96 \\
107.24 \\
71.524 \\
35.807 \\
0.091025 Min
\end{tabular}

Type: Equivdenk (von-Mises) Stress

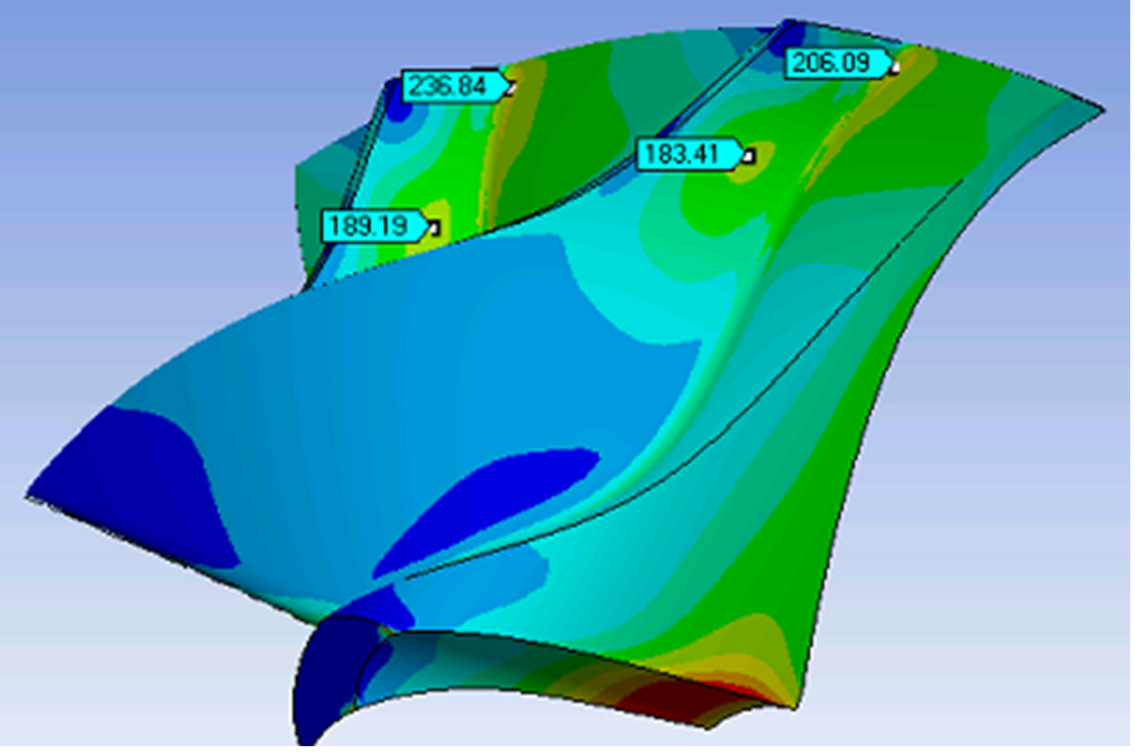

Fig. 12.5. Stress analysis of advanced MD2 compressor: high stress is located in fillet area which could be mitigate with slight increase in fillet radius without compromising aerodynamic performance 


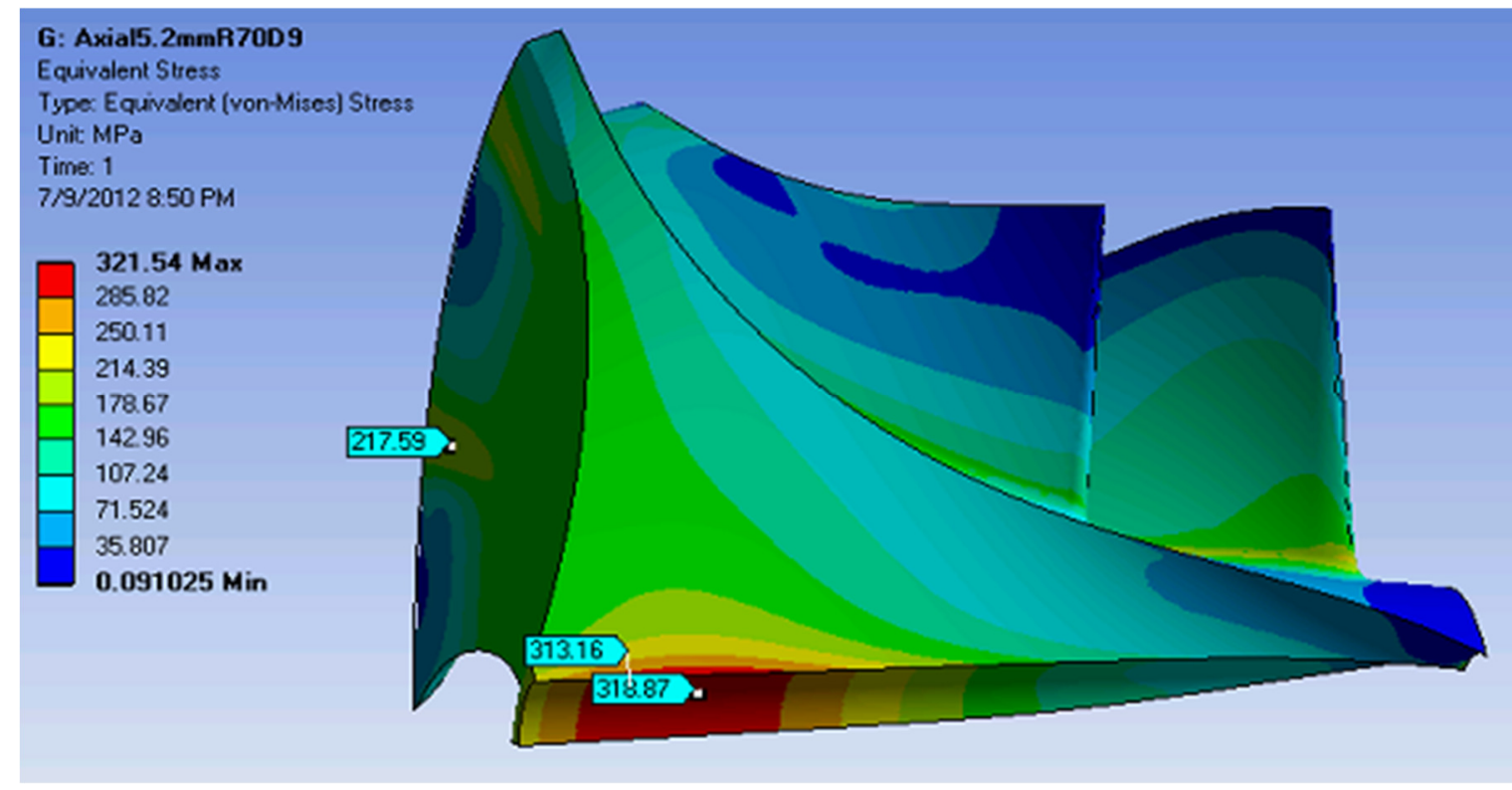

Fig. 12.6. Stress analysis of advanced MD2 compressor: the bore area stress is within design target.

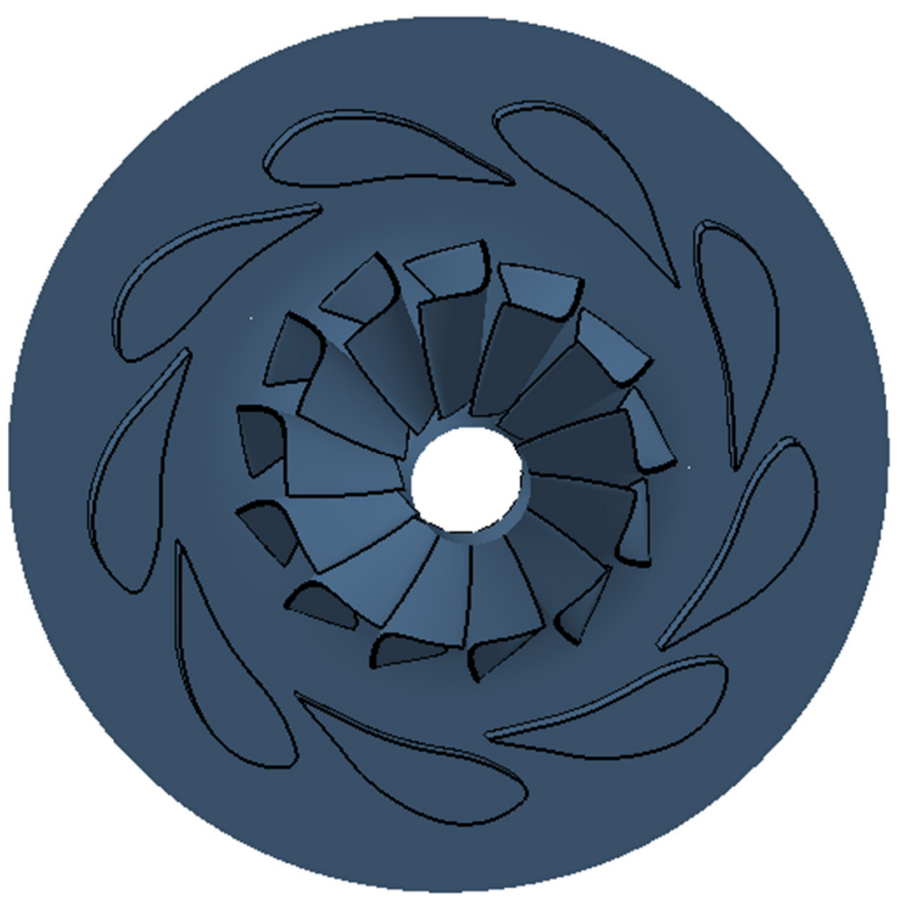

Fig. 12.7. The advanced MD2 mixed flow turbine was designed to fit into production VGT mechanism (9 nozzle vanes and 13 rotor blades shown here but final design uses 11 rotor blades)

Figoure 12.7 shows the design of the advanced MD2 mixed flow turbine that was design to fit into production donor turbine GT37 that has 9 VGT nozzle vanes. Either 13 or 11 blades are the better match with 9 nozzle vanes. After a few design and CFD/CAE iterations, 11 blades were used for better trade-off between performance, flow capacity and inertia. 


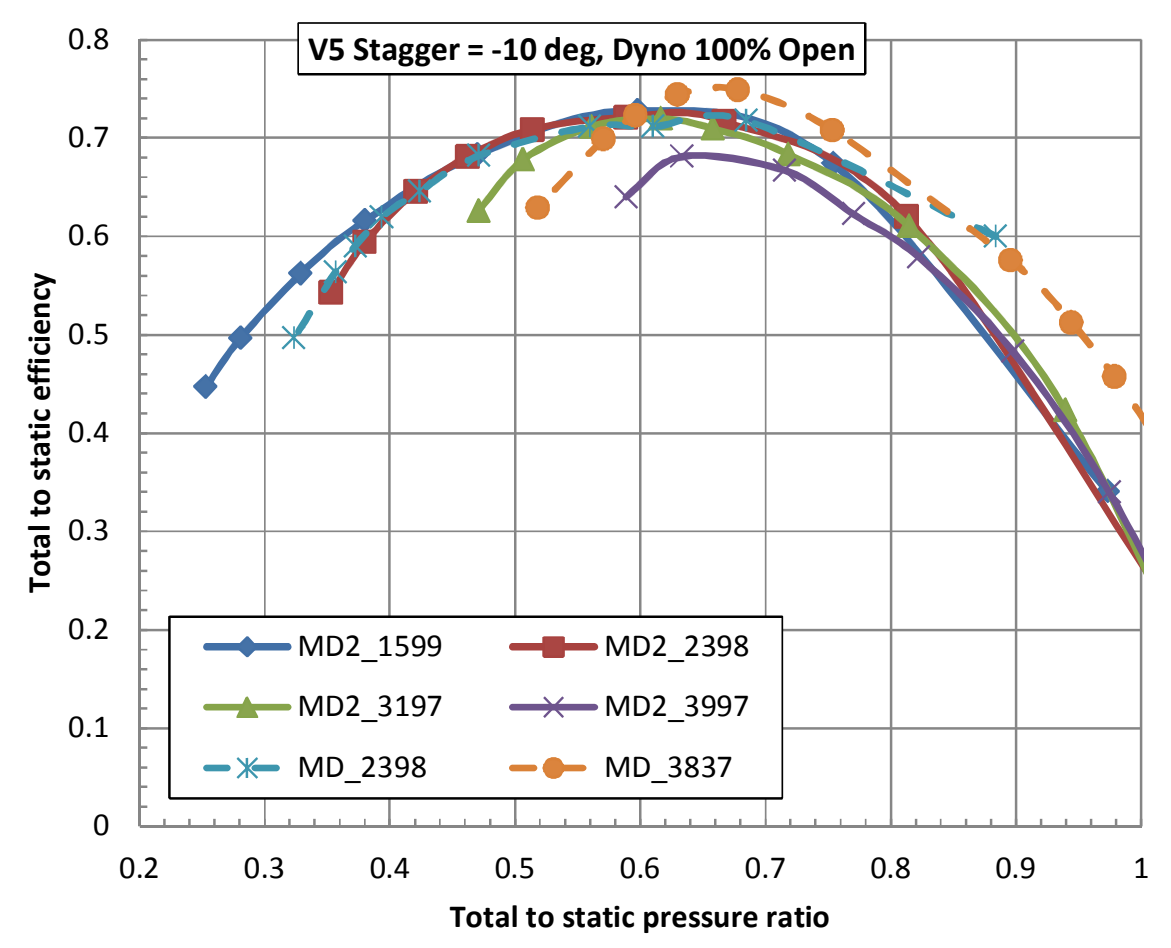

Fiq. 12.8. Meanline prediction of MD2 vs MD turbine performance at full open position

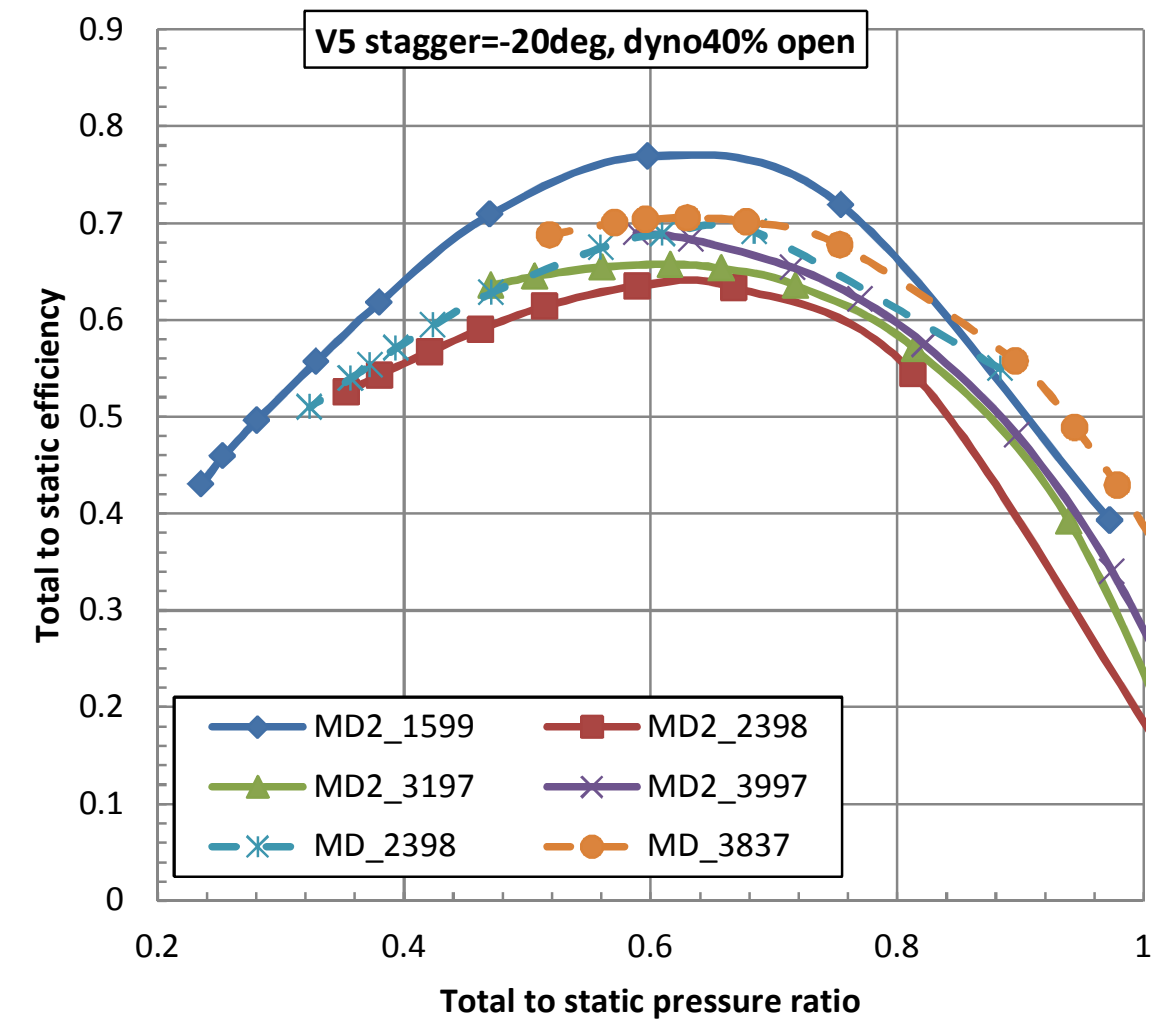

Fia. 12.9. Meanline prediction of MD2 vs MD turbine performance at partial open position 


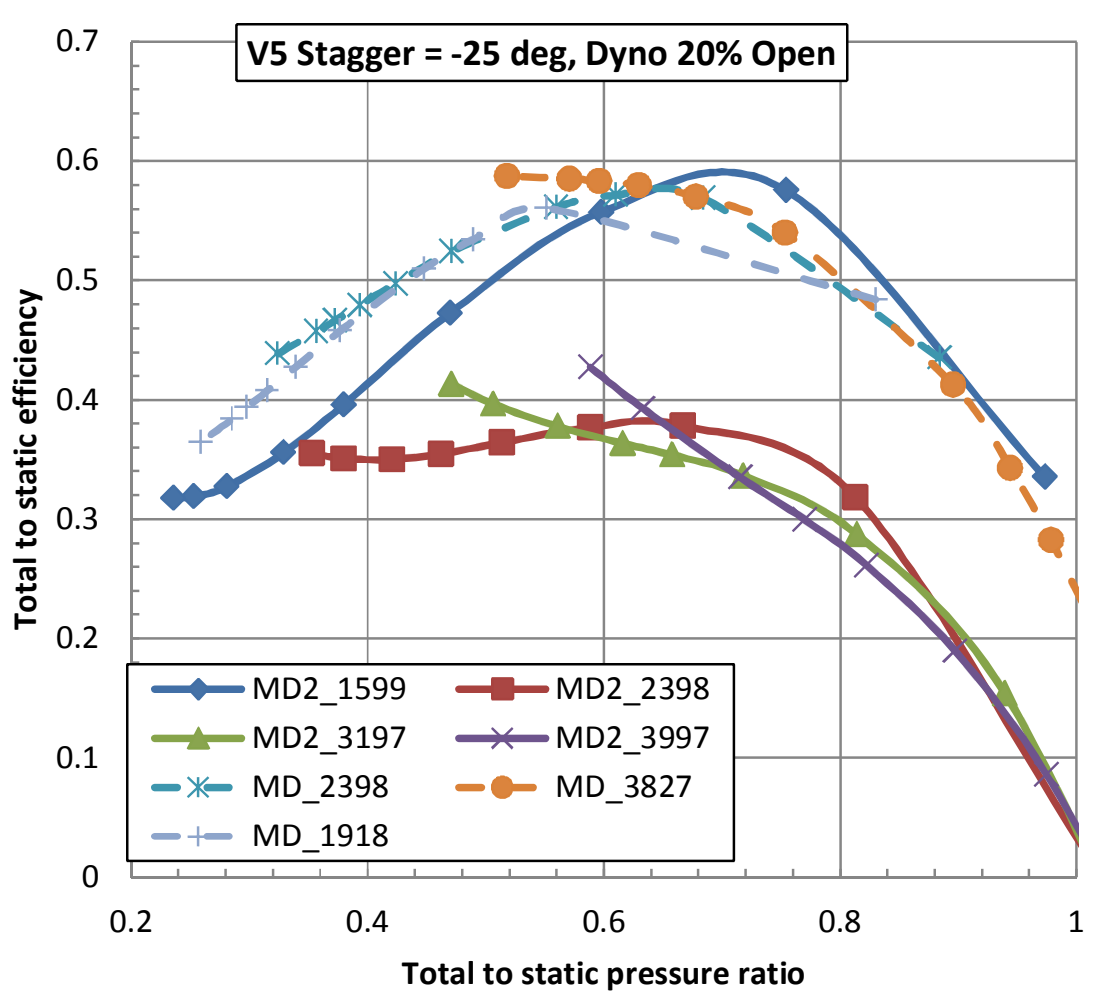

Fia. 12.10. Meanline prediction of MD2 vs MD turbine performance at full close position

The advanced MD2 mixed flow turbine, when matched to a relavely oversized compressor blades with rule surface impeller, may have an increased diameter thus narrower nozzlle vane height. As a result, the efficiency may be managed to match that of the advanced MD mixed flow turbine at full open or partial open positions (Fig. 12.8, 12.9). But the efficiency at very small vane open positions may be penalized (Fig. 12.10). Again, these conclusions were strictly based on the meanline predictions which do not include mechanical loss and accuracy at low $\mathrm{U} / \mathrm{C}$ areas are debatable, e.g. the performance at 1599 reduced speed for MD2 mixed flow turbine. The final evaluation has to be conducted on flow bench test and engine dynamometer test where pulsation exhaust flow is involved.

The Campbell diagram (Fig. 12.11) shows that the $1^{\text {st }}$ order blade flapping mode is about 4.8 times of max operation frequency and the first resonance frequency speed is $126,000 \mathrm{rpm}$ with 9 nozzle vane excitation which is below design speed but beyond normal customer driving cycles.

The (combined thermal and mechanical) stress analysis shows that the highest stress is located in the backface area (Fig. 12.12), which is $77 \%$ of the yield strength of the Inconel at 702 deg $\mathrm{C}$. This stress is considered acceptable even though the analysis method used was very conservative in the temperature boundary condition setting at welding area of the turbine wheel. 


\section{Campbell Diagram}

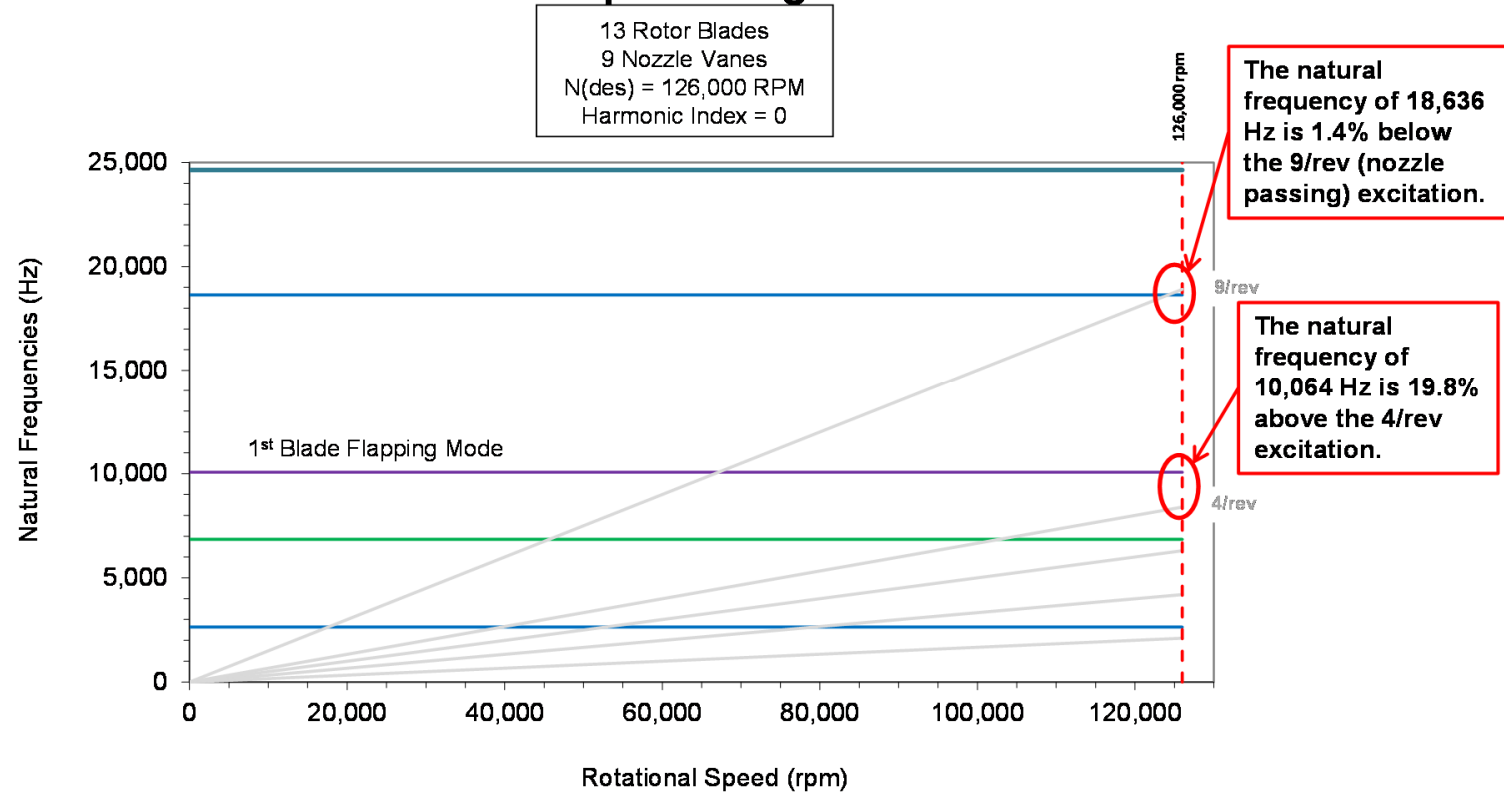

Fig. 12.11. Campbell dram of advanced MD2 turbine wheel

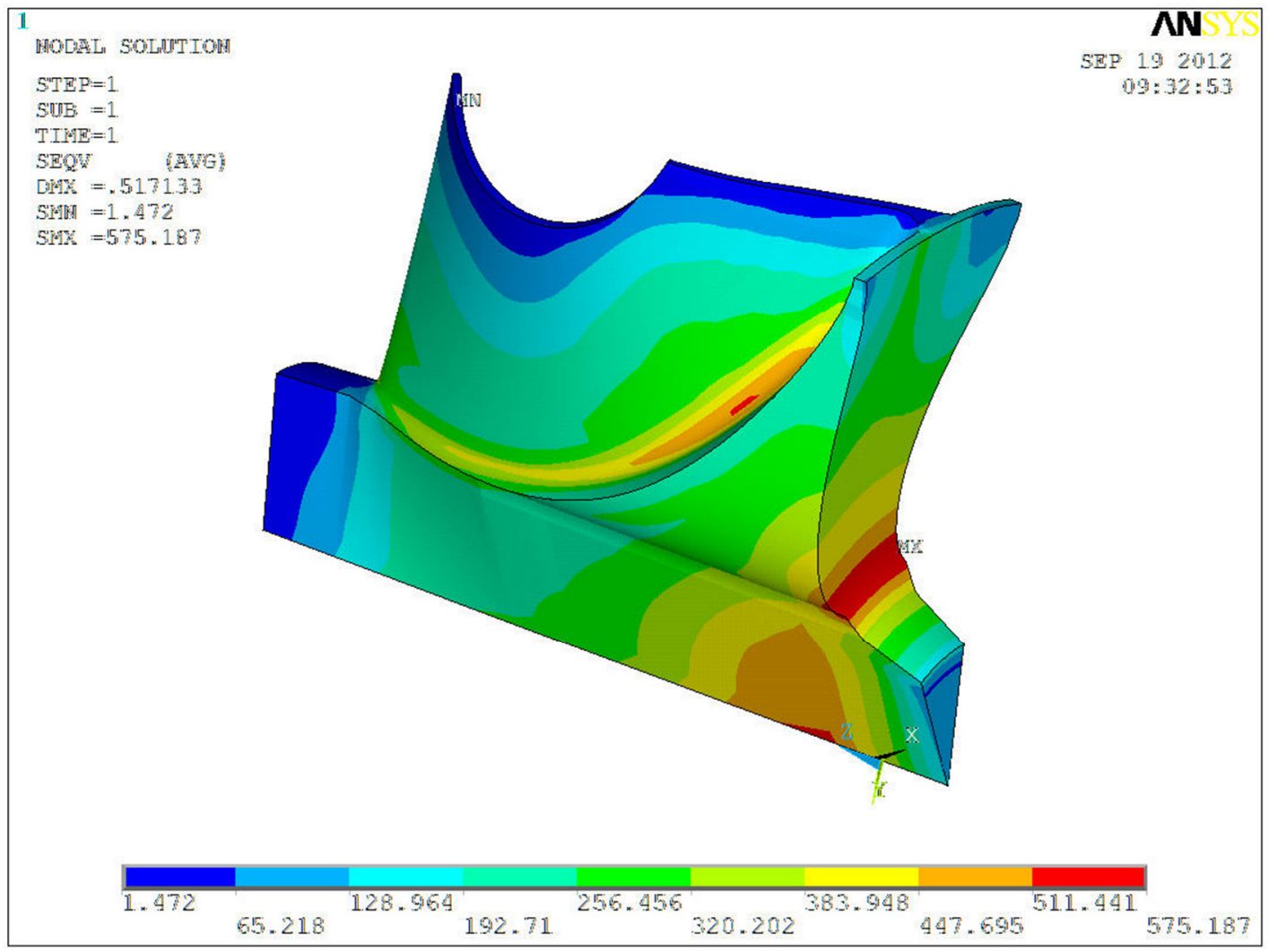

Fig. 12.12. Stress distribution of advanced MD2 turbine wheel 
12.3 Fabrication of advanced MD2 compressor and mixed flow turbine wheels

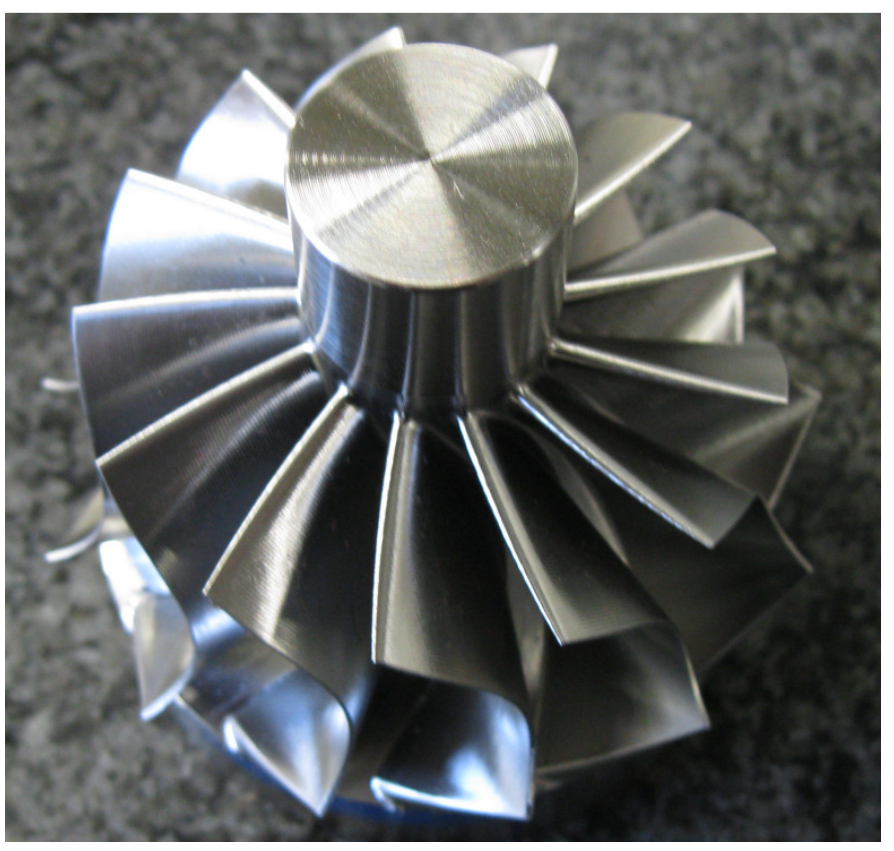

Fiq. 12.13. Fabricated advanced MD2 mixed flow turbine wheel

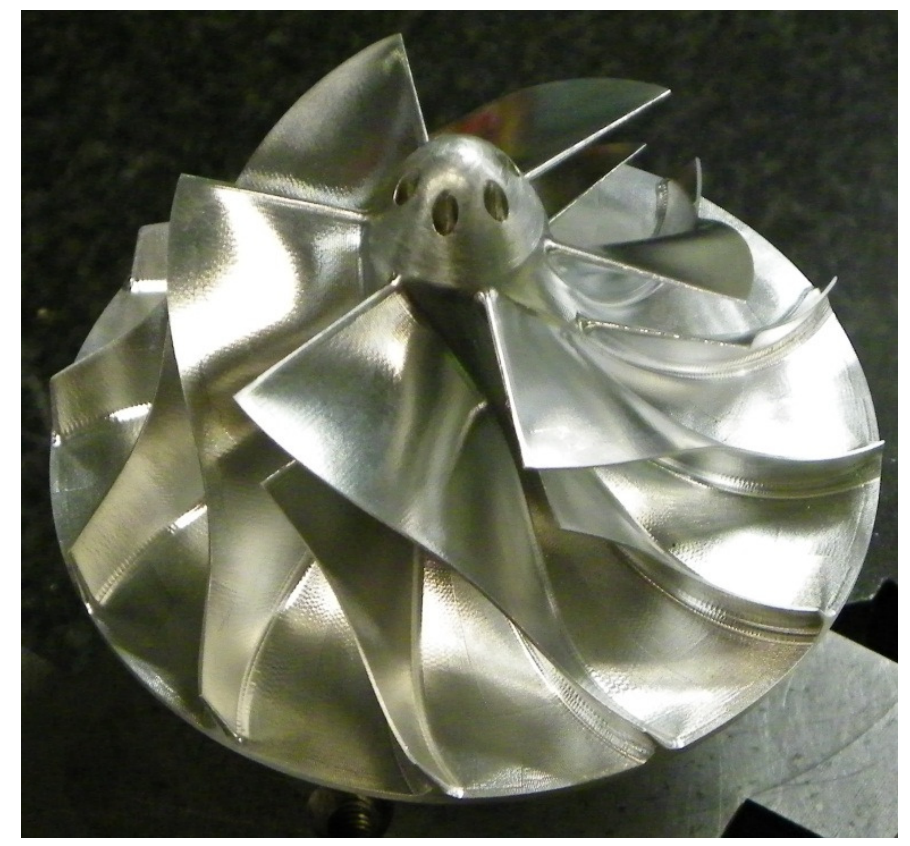

Fiq. 12.14. Fabricated advanced MD2 compressor wheel with ruled surface 


\section{Conclusions :}

- This research demonstrated that the century old turbocharger technology still has potential for further improvement, given the new application environment, new analytical and design tools;

- OEM led component research enables the technology to be optimized at system level, to be integrated into viable system solution to address future emission and fuel economy challenges, and more importantly to be evaluated in a systematic and dynamic/transient environment, contrast to isolated and single point target;

- With the financial support from DOE, by closely working with various turbocharger suppliers, Ford Motor Company and its partners successfully improved the turbocharger technology and gained important insights in the following areas:

a. Active casting treatment opens up a new design space for compressor design of high efficiency over wide operation range

b. Compressor impeller with arbitrary surface can effectively be designed for high efficiency over wide operation range with compact sizes

c. Compressor impeller with ruled surface may also be designed for high efficiency over wide operation range which is cost effective and has large wheel diameter (so called large wheel concept). The larger compressor impeller requires larger turbine wheel, which may potentially compromise, to a certain degree, the benefit of a mixed flow turbine

d. Mixed flow turbine has renewed life due to high EGR for feed gas $\mathrm{NO}_{\mathrm{x}}$ emission control, high back pressure due to aftertreatment. The mixed flow turbine does not have the highest efficiency and its benefit may not be fully appreciated without a systematic, dynamic full range (especially the light load) engine dynamometer testing.

- In this research, state-of-art analytical tools were used extensively. This analysis guided development process allows $10-18$ design iterations to be conducted, in a time and cost effective manner, for optimal compressor and turbine performances, which is incredible for a normal production cycle.

- Honeywell GT35 was used as MD donor turbo on which an advanced compressor impeller with arbitrary surface and active casing treatment, matched to an advanced mixed flow turbine, was developed, fabricated, experimentally evaluated on steady state flow bench and a MD diesel engine dynamometer. The flow bench test demonstrated that the advanced MD compressor offers 38\% extra flow capacity, 8-10\% better efficiency over the MD donor turbo compressor. The mixed flow turbine gained $10-15 \%$ efficiency at low $\mathrm{U} / \mathrm{C}$ areas. The steady state MD diesel engine test shows that the advanced MD turbo helps to have BSFC improved by $3 \%$ at selected part load and full load points, besides $30 \%$ increase in rated power, compared with the base donor turbo.

- An IHIF55 turbocharger was used as donor turbo on which another advanced compressor and mixed flow turbine wheels were designed and fabricated for a 3.2L LD diesel application. The advanced LD turbo was used to evaluate the "large turbo concept" that adopts relatively large compressor impeller with rule surface and mixed flow turbine, which shows limited improvement over donor turbo during flow bench test. However, the steady state engine test still shows that the LD turbo has 2-4\% BSFC benefit over engine part load area. The transient engine dynamometer test has demonstrated that the advanced LD turbo (TS11) gained 3.3\% thermal efficiency improvement over the base LD turbo on FTP cycle at T2B5 tailpipe emission level, which achieved the program target. 
- All the computer analyses, flow bench test and engine dynamometer test data indicated that the advanced compressor with arbitrary surface has superior performance with compact dimension, compared to the relatively large advanced compressor with ruled surface, when matched with respective mixed flow turbines. Efforts should and could be made to drastically reduce the point milling time on the machine of arbitrary surfaces with the help of the state-of-the-art high speed milling technologies.

- Both compressor and turbine are very hard to evaluate on conventional steady state turbocharger flow bench test at low mass flow areas, which unfortunately are heavily populated in US FTP cycle. Future turbocharger flow bench needs to extend the measurement range to low turbo speed, low mass flow and low turbine speed ratio $(\mathrm{U} / \mathrm{C})$ areas, to accurately quantify the impact of future turbocharger technology on engine performances.

- Both advanced MD turbocharger and LD turbocharger demonstrated 3\% or more fuel economy improvement on engine dynamometer test when pulsation exhaust flow was involved, which was more favorable to mixed flow turbine, better than what was predicted by GTPower (or other 1D engine system) simulations that were based on extrapolated steady state compressor and turbine maps.

- This project was focused on aerodynamic design optimization of compressor and turbine from the engine system and real driving cycle perspective. Additional efforts are required before the advanced turbochargers can be manufactured for mass production, e.g. durability test validation, mass production feasibility assessment, etc. 


\section{References :}

1. Aihama, K. et al, SAE2001-01-0655, "Mechanism of the smokeless rich diesel combustion by reducing temperature".

2. Akagawa, H. et al. SAE1999-01-0183, "Approaches to solve problems of the premixed lean diesel combustion"

3. Akihama, K. et al, SAE2001-01-0655, "Mechanism of the smokeless rich diesel combustion by reducing temperature"

4. Baines, N., Fundamentals of turbocharging, ConceptsNREC, 2005

5. Buchwald, R. et al, SAE2004-01-0936, "Adaptation of injection system parameters to homogeneous diesel combustion"

6. Fisher, F.B. SAE880794, "Application of map width enhancement devises to turbocharger compressor stages"

7. Gu, Ronglei, et al, "Surge Control for Centrifugal Compressor of Turbocharger", SAE2004-08-0232

8. Habermann, K. et al., Beijing International Symposium, "Optimization of the operation characteristics of turbocharged engines through compressor variability" 2006

9. Harada, A. SAE980533, "The effects of mixture formation on premixed lean diesel combustion"

10. Harada, A. et al, SAE980533, "The effects of mixture formation on premixed lean diesel combustion"

11. Hasegawa, R. et al, SAE2003-01-0745, "HCCl combustion in DI diesel engine"

12. Helmantel, A. et al, SAE2004-01-0935, "HCCI Operation of a passenger car common rail DI diesel engine with early injection of Conventional Diesel fuel"

13. Kindl, H.M. et al, SRR2004-0151 (Ford Tech Report), "Influence of various compressor inlet designs on compressor performance"

14. Ishima, T. et al, SAE2003-01-1823, "Characteristics of $\mathrm{HCCl}$ diesel combustion operated with a hollow cone spray"

15. Iwabuchi, Y. et al. SAE1999-01-0185, "Trial of new concept diesel combustionpremixed compression ignited combustion"

16. Karamanis, N. et.al. Int. J. Engine Research, Vol 3. No.3, "Mixed-flow turbines for automotive turbochargers: steady and unsteady performance", 2002

17. Kyrtatos, N., and Watson, N., 1980, "Application of Aerodynamically Induced Prewirl to a Small Turbocharger Compressor," ASME Paper 80-GT-23

18. Kimura, S. et al, SAE2001-01-0200, "Ultra-clean combustion technology combining a low-temperature and premised combustion concept for meeting future emission standards"

19. Najt, P.M.et al, SAE830264, "Compression-ignited homogeneous charge combustion"

20. Olsson, J. et al, SAE2004-01-0940, "Boosting for high load HCCl"

21. Onishi, S. et al, SAE790501, "Active thermo-atmospheric combustion (ATAC) -A new combustion process for internal combustion engines"

22. Pampreen, R. C, Compressor Surge and Stall, ConceptsNREC, 1993

23. Ryan, T.W. SAE961160, "Homogeneous charge ignition of diesel fuel"

24. Sun, R. et al, SAE2004-01-0933, "An HCCl engine: power plant for a hybrid vehicle"

25. Uchida, $H$. et al, R\&D Review of Toyota CRDL Vol. 41, NO.3, "Development of wide flow range compressor with variable inlet guide vane", 2006

26. Yanagihara, $\mathrm{H}$. et al, "A simultaneous reduction of NOx and soot in diesel engines under a new combustion system (Uniform Bulky Combustion System -UNIBUS)", $17^{\text {th }}$ International Vienna Motor Symposium, 1996

27. Yoshinaka, T. et al, SAE961163, "Emission characteristics of premixed lean diesel combustion with extremely early staged fuel injection" 
28. Kook, S. and C. Bae, "Combustion control using two-stage diesel fuel injection in a single cylinder PCCI engine, SAE2004-01-0938, 2004

29. Yang, J., et al, "boosted internal combustion engines and air compressors used therein," US Patent 6,651,431B1

30. Kui Jiao, Harold Sun, Xianguo Li, Hao Wu, Eric Krivitzky, Tim Schram, Louis M. Larosilier "Numerical simulation of air flow through turbocharger compressors with dual volute design," Applied Energy, Vol. 86 (2009) 2494-2506

31. A century of turbocharging, ABB Turbo Systems, Ltd., http://www.swissships.ch/berichte-buecher/berichte/ABB Turbo Systems/TM 2005 100Y EN.pdf

32. N. Watson, and R. S. Janota, Turbocharging the internal combustion engine, 1982, MacMillan, London

33. F. J. Wallace, "A systematic approach to the design of radial inflow and mixed flow turbines," NACA Report, No. CP 1180

34. A. Whitefield and N.C. Baines, Design of Radial Turbomachines, 1990, Longman, England

35. US Patent 2011/0173975 A1, "Turbocharger"

36. Ron Aungier, Centrifugal Compressors: A Strategy for Aerodynamic Design and Analysis, ASME, 2000

37. Xiao J, Xu W, Gu CG. Self-recirculating casing Treatment for a radial compressor. Chinese Journal of Mechanical Engineering, 2009, 22(N4), 567-573.

38. Sivagnanasundaram, S., Spence, S., Early, J. and Nikpour, B. An investigation of compressor map width enhancement and the inducer flow field using various configurations of shroud bleed slot. ASME paper, GT2010-22154, 2010.

39. Sivagnanasundarm, S., Spence, S., Early, J. and Nikpour, B. An impact of various shroud bleed slot configurations and cavity vanes on compressor map width and the inducer flow field. ASME paper, GT2011-45629, 2011.

40. Zhang JZ, Sun H, Hu LJ and He H. Fault Diagnosis and Failure Prediction by Thrust Load Analysis for a Turbocharger Thrust Bearing. ASME paper, GT2010-22320.

41. Yang $C$, Chen S, Li D et.al. Inlet recirculation influence to the flow structure of centrifugal compressor. Chinese Journal of Mechanical Engineering, 2010, 23(N4), 1-7

42. Hunziker, R., Dickmann, H-P and Emmrich, R. Numerical and Experimental Investigation of a Centrifugal Compressor with an Inducer Casing Bleeding System. Proceedings of the Institution of Mechanical Engineers Part A: Journal of Power and Energy, 2001, 215(D8), 783-791.

43. H. Sun, "Advanced boost system development for diesel $\mathrm{HCCl} / \mathrm{LTC}$ applications," US DOE Annual Merit Review, Washington, D.C., May 18, 2012

44. Liangjun $\mathrm{Hu}$, Investigation of operability margin improvement in an advanced turbocharger centrifugal compressor for $\mathrm{HCCl}$ diesel engine., Ph.D. Dissertation, Beijing Institute of Technology, 2010

45. Daisaku Sakaguchi, Keiichi Nagoshi, Motoki Tanimura, Masahiro Ishida and Hironobu Ueki, "Effect of Guide Vane in Ring Groove Arrangement for a Small Turbocharger," American Institute of Physics, The $10^{\text {th }}$ Asian international conference on fluid machinery June 28, 2010 -- Volume 1225, pp. 47-54

46. Hamerick, J. T., Ginsburg, A., and Osborn, W. M., 1950, "Method of Analysis for Compressible Flow through Mixed-Flow Centrifugal Impellers of Arbitrary, Design," NACA Report No.TN 2165

47. Chen, H., and Baines, N. C., "Analytical optimization design of radial and mixed flow turbines," Proc. Inst. Mech. Eng., Part A, 206, pp177-187, 2992

48. Harold Sun, Yong Shu, Dave Hanna, Tim Schram, "Control strategy for decreasing resonance in a turbocharger," US Patent 20110023479A1

49. Harold Sun, et. al., "SWITCHABLE DUAL-PORT CASING TREATMENT SCHEME FOR ENHANCED TURBOCHARGER COMPRESSOR OPERATING RANGE”, Journal of Automobile Engineering, Feb. 2013 
50. Wallace, F.J. and Pasha, S.G.A., 1972, "Design, construction and testing of a mixed flow gas turbine," The $2^{\text {nd }}$ International JSME Symposium on fluid machinery and fluids, Tokyo, Japan, pp. 213-224

51. Ron Anungier, Turbine Aerodynamics: Axial-flow and radial inflow turbine design and analysis, ASME Press, 2006 